\title{
THEORY, SIMULATION, FABRICATION AND TESTING OF DOUBLE NEGATIVE AND EPSILON NEAR ZERO METAMATERIALS FOR MICROWAVE APPLICATIONS
}

\author{
A thesis \\ Presented to the faculty of California Polytechnic State University, \\ San Luis Obispo
}

\author{
In Partial Fulfillment \\ of the Requirements for the Degree \\ Master of Science in Electrical Engineering
}

By

Neil Patel

June 2008 


\section{AUTHORIZATION FOR REPRODUCTION OF MASTER'S THESIS}

I grant permission for the reproduction of this thesis or any of its parts, without further authorization from me.

\begin{tabular}{l} 
Neil Patel \\
TUNE $16 \quad 2008$ \\
\hline
\end{tabular}

Date 


\section{APPROVAL PAGE}

TITLE:

THEORY, SIMULATION, FABRICATION AND TESTING OF DOUBLE NEGATIVE AND EPSILON NEAR ZERO METAMATERIALS FOR MICROWAVE APPLICATIONS

AUTHOR: Neil Patel

DATE June $6^{\text {th }} 2008$

SUBMITTED:

will 6,2008

Date

June 6,2008

Date

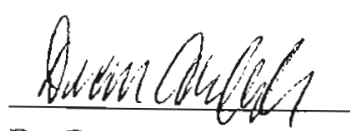

Dr. Dean Arakaki, Chair

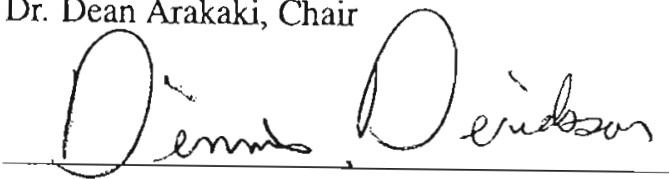

Dr. Dennis Derickson
June 6,2008

Date 


\begin{abstract}
THEORY, SIMULATION, FABRICATION AND TESTING OF DOUBLE NEGATIVE AND EPSILON NEAR ZERO METAMATERIALS FOR MICROWAVE APPLICATIONS
\end{abstract}

\author{
Neil Patel \\ Electrical Engineering Department \\ Master of Science
}

Natural structures exhibiting simultaneous negative bulk permittivity and permeability have not yet been discovered. However, research interest over the past five years has grown with the proposition that artificial structures exhibiting these properties are realizable using specially-designed metallic inclusions embedded in host dielectric bodies. A periodic structure of metallic inclusions much smaller than the guided wavelength and embedded in a host dielectric medium is known in the physics and microwave communities as a "metamaterial". Such frequency-dependent effectively homogeneous materials may be designed to exhibit negative permeability and permittivity at certain frequencies. As predicted by electromagnetic theory, such negative index or "left-handed" metamaterials are shown to have unique filtering properties and exhibit negative refraction and "backward wave" propagation. The "backward wave" phenomenon describes the anti-parallel nature of phase velocity and group velocity in a negative index metamaterial and can be additionally characterized in vector theory using the left hand rule. Additionally, "epsilon-near zero" (ENZ) metamaterials are characterized by a bulk permittivity equal to zero. Applications include focusing radiation emitted by small apertures. 
This thesis provides the theory for metamaterial structures supported by simulations conducted with the commercial finite element method solver: Ansoft HFSS. Metallic inclusions such as the split ring resonator structure (SRR), S-shaped split ring resonator ( $S$ SRR), wire rod and capacitively loaded strip (CLS) are presented analytically and simulated in HFSS. Metamaterial structures designed to exhibit left-handed behavior in the X-band frequency region are simulated for frequency-dependent transmission, reflection and refractive properties. A test configuration for measuring a metamaterial slab's match to free space is proposed and constructed. Additionally a prism design and test plan geared for anechoic chamber testing and refraction measurement is proposed and built. Simulated inclusions are fabricated on FR-4 epoxy laminate boards, combined to form metamaterial structures, and tested in the Cal Poly Anechoic chamber. Results show that transmission properties match closely with HFSS simulations. Prism metamaterial testing shows that negative refraction is visible in the 8 to $9 \mathrm{GHz}$ region. A modified form of the Nicolson Ross-Weir method for parameter extraction using S-parameter data is shown to provide an initial approximation for the permeability and permittivity of the structure under test. Finally, both negative and zero-index metamaterials are analyzed in HFSS simulations to improve the directivity of EM radiation from sub-wavelength apertures. Epsilon-near zero metamaterials placed on sub-wavelength apertures are shown to improve directivity by two fold in the far-field at design frequencies. 


\section{ACKNOWLEDGMENTS}

I would like to Acknowledge Professor Dean Arakaki, whose guidance was instrumental in the completion of this project. Without his willingness to donate time and effort in obtaining grants for materials and the construction of the Cal Poly Anechoic Chamber this project would not have been possible. I would also like to acknowledge committee members Dr. Dennis Derickson and Dr. Xiaomin Jin for the time taken out of their busy schedules to give project feedback and learn about the exciting field of metamaterials. Finally, I would like to acknowledge my parents Ajay and Shama Patel; without their love and support the past six years, my completion of a Master of Science in Electrical Engineering would not have been possible. 


\section{Contents}

List of Figures $\quad$ x

List of Tables

1 Introduction 1

1.1 Metamaterials . . . . . . . . . . . . . . . . . . . . 1

1.1.1 Basic plane wave theory . . . . . . . . . . . . . . . 2

1.2 Double Negative Index metamaterials . . . . . . . . . . . . . . . 3

2 Parameter Extraction 9

2.1 Nicolson-Ross-Weir method . . . . . . . . . . . . . . . . . . . 9

2.1.1 Complex propagation constant of the material under test . . . . . . . . 12

2.1.2 Intrinsic parameters for a Coaxial Waveguide . . . . . . . . . . . . . . 13

2.1.3 Intrinsic parameters for a Rectangular Waveguide . . . . . . . . . . . . 14

2.2 An implementation of the NRW method pertaining to Double Negative Metamaterials 15

2.3 Divergence in the Nicolson-Ross-Weir equations . . . . . . . . . . . 18

3 The Split Ring Resonator Inclusion $\quad 20$

3.1 Split Ring Resonators and Permeablility . . . . . . . . . . . . . . . . . . . 20

3.1 .1 Array of cylinders . . . . . . . . . . . . . . . . . 21

3.1.2 Capacitive array of sheets wound on cylinders . . . . . . . . . . 23

3.1 .3 Split ring resonator . . . . . . . . . . . . . . . . 25

3.2 Circular SRR inclusion simulation in Ansoft HFSS . . . . . . . . . . . 27

3.2 .1 Unit cell synthesis in HFSS . . . . . . . . . . . . . . 27

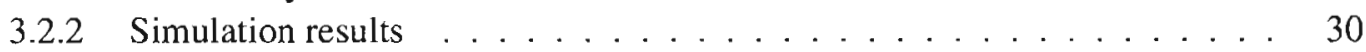

3.2 .3 Parameter variations . . . . . . . . . . . . . . . . . . 30

3.3 Square / Quadrilateral SRR inclusion simulation in Ansoft HFSS . . . . . . . . . 34

3.3.1 Unit cell synthesis in HFSS . . . . . . . . . . . . . . . . . . . . 34

3.3 .2 Parameter variations . . . . . . . . . . . . . . . 34

4 Wire and strip structures to realize negative permittivity 38

4.1 Thin wire structures . . . . . . . . . . . . . . . . . . . . . . . . . . . 39

4.2 Capacitively loaded strips $(\mathrm{CLS}) \ldots \ldots \ldots \ldots \ldots \ldots \ldots$ 
5 The S-shaped Split Ring Resonator Inclusion (S-SRR) 46

5.1 S-shaped SRR inclusion simulations . . . . . . . . . . . . . . . 51

5.1 .1 Unit cell Synthesis . . . . . . . . . . . . . . . . . . . . 51

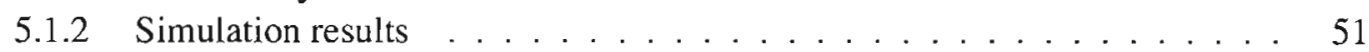

5.1 .3 Parameter Variations . . . . . . . . . . . . . . . . 52

5.2 Summary of metallic inclusions $\ldots \ldots \ldots \ldots \ldots$

6 Parameter Extraction applied to Discrete Metallic inclusions 57

6.1 Extraction results from HFSS simulations . . . . . . . . . . . . . 57

6.1.1 Circular SRR with thin wire strips . . . . . . . . . . . . . 57

6.2 Square SRR with capacitively loaded strips . . . . . . . . . . . . . . 60

6.3 Capacitively loaded strips . . . . . . . . . . . . . . . . . . 63

7 Copper inclusion parametric analysis and optimization $\quad 66$

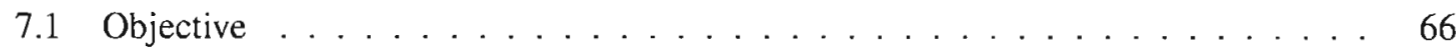

$7.2 \quad$ SRR Parameters . . . . . . . . . . . . . . . . . . . . . . . . . 67

7.3 Parameteric sweep results . . . . . . . . . . . . . . . . . . 67

7.3.1 Square/Quadrilateral SRR - Diameter sweep . . . . . . . . . . . 67

7.4 Planar versus non-planar designs . . . . . . . . . . . . . . . . . 70

7.5 S-SRR Parameters . . . . . . . . . . . . . . . . . . 71

7.6 Final Metamaterial transmission simulations . . . . . . . . . . . . . 73

8 Metamaterial prism simulation in HFSS

8.1 Simulation setup . . . . . . . . . . . . . . . . 76

8.2 Ideal simulations . . . . . . . . . . . . . . . . . . 77

8.3 Simulation with metal inclusions . . . . . . . . . . . . . . 80

9 Final metamaterial structure designs $\quad \mathbf{8 2}$

9.1 SRR/CLS structure . . . . . . . . . . . . . . . . . . . . . 82

9.2 S-SRR Structure . . . . . . . . . . . . . . . . . . . . . . . . . . . . . . 83

9.3 Prism structure . . . . . . . . . . . . . . . . . . . . . 84

10 Test setup $\quad 88$

10.1 Test Setup in Anechoic Chamber f . . . . . . . . . . . . . . 88

10.2 Test Setup characterization . . . . . . . . . . . . . . . . . . . . . 92

10.3 Test plan . . . . . . . . . . . . . . . . . . . . . 96

10.3 .1 Transmission testing . . . . . . . . . . . . . . . . 96

10.3.2 Metamaterial refraction testing . . . . . . . . . . . . . . . . 96

11 Test results $\quad 98$

11.1 Transmisson testing . . . . . . . . . . . . . . . . . . . . . . . . . . . . . . . 98

11.1 .1 S-SRR metamaterial . . . . . . . . . . . . . . . . . . 98

11.1 .2 SRR/CLS structure . . . . . . . . . . . . . . . . . . 104

11.2 Metamaterial prism refraction testing . . . . . . . . . . . . . 105

11.2.1 FR-4 only metamaterial prism for test setup confirmation . . . . . . . . 110 
12 Application: Directivity and Power Enhancement through sub-wavelength apertures 113

12.1 Theory . . . . . . . . . . . . . . . . . . . . 113

12.2 HFSS simulations . . . . . . . . . . . . . . . . . . 115

12.2 .1 Ideal simulations . . . . . . . . . . . . . . . . . . . 115

12.2.2 Metallic inclusions embedded in exit face dielectric slab . . . . . . . . . 121

13 Conclusions $\quad 130$

A Matlab Code 132

A.1 Theoretical simulation of circular split ring resonator (SRR) $\ldots \ldots \ldots \ldots \ldots$

A.2 Theoretical simulation of $S$-shaped split ring resonator (SRR) . . . . . . . . . 134

A.3 Parameter extraction using Ziolkoswki's modified NRW relations . . . . . . . 136

A.4 Parameter extraction using Chen et al's modified NRW relations . . . . . . . . . 139

B Analysis of Project Design 142

B.1 Summary of Functional Requirements . . . . . . . . . . . . . . . . 142

B.2 Primary constraints . . . . . . . . . . . . . . . . . . . 142

B.3 Economic . . . . . . . . . . . . . . . . . . . . . 143

B.3.1 Bill of Materials . . . . . . . . . . . . . . . . . . 143

B.4 Environmental . . . . . . . . . . . . . . . . . . . . . . . 143

B.5 Manufacturability . . . . . . . . . . . . . . . . . . . . . . 144

B.6 Sustainability . . . . . . . . . . . . . . . . . . . . . . . 144

B.7 Ethical . . . . . . . . . . . . . . . . . . . . . . . . 144

B.8 Health and safetey . . . . . . . . . . . . . . . . . . . . . . . 144

B.9 Social and political . . . . . . . . . . . . . . . . . . . . . 144

B.10 Development . . . . . . . . . . . . . . . . . . . . . 144

$\begin{array}{ll}\text { Bibliography } & 146\end{array}$ 


\section{List of Figures}

1.1 Current sheet at $x=x_{0}$ radiates into a left handed medium Source: D.R.Smith, Duke University. . . . . . . . . . . . . . . . . . . . .

1.2 Depiction of refraction according to Snell's law. Negative refractive materials show refraction on the other side of the normal. Snell's law is clearly preserved but the angle for $\theta_{2}$ is negative. . . . . . . . . . . . . . . .

1.3 Refraction in a slab of left-handed/Double negative material. The negative refractive index causes the bending of rays to negative angles of the surface normal. The diverging beams of a point source convege back towards a focal point. The first focal point may form inside the slab if the material has sufficient thickness. . . . .

1.4 Simulation in Ansoft HFSS of a parallel plate waveguide. The direction of phase velocity is shown with arrows. It can be seen in increasing the phase from $0^{0}$ to $180^{\circ}$ that the phase velocity is opposite in the middle material is opposite to the materials on either end. . . . . . . . . . . . . . . .

1.5 Simulation in Ansoft HFSS of the lens/negative refractive attributes of double negative/left handed media. . . . . . . . . . . . . . . . .

2.1 A filled waveguide undergoing excitation encounters multiple reflections at the airsample interfaces. . . . . . . . . . . . . . . . . .

3.1 An array of metallic cylinders with an external magnetic field applied parallel to the cylinders. . . . . . . . . . . . . . . . . . . . .

3.2 The cylinders are now seen to have an internal structure. The sheets are wound in a split ring fashion in such a way that current cannot flow freely. However, if the distance, $d$ is small enough, current may be induced. An increase in capacitance between sheets relates to an increase in current. Reference: [17] . . . . . . . . 23

3.3 A thin flat disk shaped split ring resonator. . . . . . . . . . . . . . 25

3.4 A circular split ring resonator with the variables: $a=10 \mathrm{~mm}, c=1 \mathrm{~mm}, d=0.1 \mathrm{~mm}$, $\mathrm{l}=2 \mathrm{~mm}$ and $\mathrm{r}=2 \mathrm{~mm}$ is evaluated in Matlab using the effective permeability relation (3.16) on the left. The equation predicts a resonance in permeability at approximately $13.5 \mathrm{GHz}$. HFSS simulations to obtain S-paramaters and subsequent paramater extraction predicts a resonance in the real part of the permeability at $11 \mathrm{GHz}$ (right plot). HFSS simulations of a circular SRR are presented in more detail in section 3.2. . . . . . . . . . . . . . . . . . .

3.5 Outline of a complete metamaterial slab. . . . . . . . . . . 28 
3.6 Single metamaterial cell PEC/PMC wavegulde configuration in HFSS. The waveport integration lines are shown. PEC/PMC faces are also indicated. . . . . . . . .

3.7 S-paramaters obtained from HFSS of a circular SRR with parameters: $r=1.4 \mathrm{~mm}$, trace width $=0.5 \mathrm{~mm}$, gap width $=0.1 \mathrm{~mm}$, lattice spacing (dielectric spacing) $=$ $1 \mathrm{~mm}$ and substrate dielectric permittivity $=2.2 \ldots \ldots \ldots \ldots \ldots$

3.8 Resonant Frequency of circular SRR for multiple outer radius lengths. . . . . . . . 32

3.9 Resonant Frequency of circular SRR for varying substrate dielectric constants. SRR outer radius kept constant at $2.5 \mathrm{~mm}$. Trace width is $0.5 \mathrm{~mm}$. Gap sizing is $0.1 \mathrm{~mm}$. Unit cell boundaries unchanged at $5 \mathrm{~mm} \times 5 \mathrm{~mm}$ with a dielectric height of $1 \mathrm{~mm}$. . .

3.10 Resonant Frequency of circular SRR for varying copper trace widths. SRR outer radius kept constant at $4.0 \mathrm{~mm}$. Gap sizing is $0.1 \mathrm{~mm}$. Unit cell boundaries unchanged at $5 \mathrm{~mm} \times 5 \mathrm{~mm}$ with a dielectric height of $1 \mathrm{~mm}$. The Dielectric is Rogers Duroid

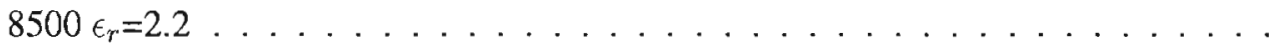

3.11 Resonant Frequency of circular SRR for varying gap widths. SRR outer radius kept constant at $4.0 \mathrm{~mm}$. Trace width is $0.5 \mathrm{~mm}$. Unit cell boundaries unchanged at $5 \mathrm{~mm}$ x $5 \mathrm{~mm}$ with a dielectric height of $1 \mathrm{~mm}$. The Dielectric is Rogers Duroid $8500 \epsilon_{r}=2.234$

3.12 Single unit cell of a square SRR in the PEC/PMC waveguide configuration for simulation in HFSS. . . . . . . . . . . . . . . . . . . 35

3.13 Resonant Frequency of a square SRR with multiple outer radius lengths. . . . . 36

3.14 Resonant Frequency of square SRR for varying copper trace widths. . . . . . . . . 36

3.15 Resonant Frequency of square SRR for varying gap widths. . . . . . . . . . 37

4.1 HFSS structure example for an array of thin wire rods. . . . . . . . . . . . 41

4.2 Transmission results from a $3 \times 3$ wire rod array simulated in HFSS. Cylinder radius $=0.2 \mathrm{~mm}$. Lattice spacing, $a=5 \mathrm{~mm}$, and the dielectric medium is air. Resonance is seen at $20 \mathrm{GHz}$. The location of the plasma frequency is thus at $\omega_{p}=20 \mathrm{GHz}$. . . .

4.3 Unit cell consisting of $2 \times 2$ array of capacitive loaded strips in PEC/PMC waveguide configuration. . . . . . . . . . . . . . . . . . .

4.4 Simulated S-parameter data on a $2 \times 2$ array of CLS structures. The low resonance is at $4.4 \mathrm{Ghz}$ and the plasma frequency where evanescent modes no longer dominate is $25.7 \mathrm{GHz}$. CLS height $(\mathrm{h})=9.6 \mathrm{~mm}$ and capacitive strip width $(\mathrm{w})=4.5 \mathrm{~mm}$. Microstrip width $=0.4 \mathrm{~mm}$. Lattice spacing $(\mathrm{a})=2.5 \mathrm{~mm} . \ldots \ldots . . . . .44$

$5.12 \mathrm{D}$ and $3 \mathrm{D}$ diagrams of $\mathrm{S}$-shaped SRR structure. . . . . . . . . .

5.2 A synthesized S-SRR unit cell in HFSS. Copper thickness is $1 \mathrm{oz}(0.2 \mathrm{~mm}) . F_{1}=F_{2}$ in this case. . . . . . . . . . . . . . . . . . . 52

5.3 Resonant Frequency of S-SRR for varying inclusion heights. . . . . . . . . 53

5.4 Resonant Frequency of S-SRR for varying inclusion widths. . . . . . . . . . . . 54

5.5 Resonant Frequency of S-SRR for varying dielectric permittivities. SRR height = $6 \mathrm{~mm}$, SRR width $=4 \mathrm{~mm}$, copper width $=0.5 \mathrm{~mm}$, spacing between mirrored $S$ shaped resonators $(\mathrm{d})=1.5 \mathrm{~mm}$, distsance between $S$-shape pairs, $\mathrm{l}=2.5 \mathrm{~mm}$. Unit cell height $(\mathrm{a})=10 \mathrm{~mm}$ and width $(\mathrm{b})=7 \mathrm{~mm} \ldots \ldots \ldots \ldots . \ldots . \ldots 54$

5.6 Divergence/resonance simulation in matlab for an S-SRR unit cell with height $=7.5 \mathrm{~mm}$, width $=4 \mathrm{~mm}$, copper width $=0.5 \mathrm{~mm}$, distance between mirrored rings $=0.5 \mathrm{~mm}, \mathrm{~S}$ SRR pair seperation, $\mathrm{l}=1 \mathrm{~mm}$, unit cell: $10 \mathrm{~mm} \times 5 \mathrm{~mm} . \ldots \ldots$ 
6.1 A unit cell with a circular SRR and thin wire strip. The boundaries are $10 \mathrm{~mm} x$ $10 \mathrm{~mm}$ in the $X$ and $Y$ directions. The separation between the SRR and wire strip is $1 \mathrm{~mm}$. SRR outer radius is $3 \mathrm{~mm}$ with a copper strip width of $1 \mathrm{~mm}$ and gap width of $0.2 \mathrm{~mm}$.

6.2 Resonance shown in scattering parameters at approximately $18 \mathrm{Ghz} . \ldots . . .58$

6.3 Extracted real part of ( $\epsilon_{r}$ from S-parameters. The equations are used respectively. . 59

6.4 Extracted real part of $\mu_{r}$ from $\mathrm{S}$-parameters. . . . . . . . . . . . . . . . 59

6.5 Extracted real part of the index of refraction and slab electrical thickness. . . . . . 60

6.6 A unit cell with a square SRR and capacitively loaded strip. The boundaries are 210mils $x$ 160mils in the $X$ and $Y$ directions. The separation between the Square $\mathrm{SRR}$ and the CLS is $0.45 \mathrm{~mm}$. SRR outer radius is $50 \mathrm{mils}$ with a copper strip width of $10 \mathrm{mils}$ and gap width of 5 mils. Rogers Duroid, $\epsilon_{r}=2.2$ is used as the dielectric substrate. . . . . . . . . . . . . . . . . . 61

6.7 Resonance shown in scattering parameters at approximately $8 \mathrm{GHz} . \ldots \ldots 1$

6.8 Extracted $\epsilon_{r}$ from S-parameters. The equations (2.29) (2.31) are used respectively. . 62

6.9 Extracted $\mu_{r}$ from $S$-parameters recorded through simulation of square SRR/CLS unit cell. . . . . . . . . . . . . . . . . . . . . . 62

6.10 Extracted index of refraction and electrical thickness from S-parameters recorded through simulation of square SRR/CLS unit cell. . . . . . . . . . . 63

6.11 Side view of the CLS $2 \times 2$ array unit cell in HFSS. $h=15.7 \mathrm{~mm}, \mathrm{~h}=4.5 \mathrm{~mm}$, and lattice spacing a $=5 \mathrm{~mm}$. The copper inclusions are embedded in an air box. . . . 63

6.12 Extracted $\mu_{r}$ from S-parameters. The equation (2.28) is used for extraction. . . . . 64

6.13 Extracted $\epsilon_{r}$ from S-parameters recorded through the simulation of a $2 \times 2$ CLS array. 65

6.14 Extracted index of refraction from $S$-parameters recorded through simulation of a $2 \times 2$ CLS array. . . . . . . . . . . . . . . . . . 65

7.1 Standard square SRR unit cell. . . . . . . . . . . . . . . . . 67

7.2 Parametric Analysis results for square SRR on FR4 substrate with 'a' varied from 70 to 150 mils in 5 mil steps. ' $c$ ' $=10$ mils ' $d$ ' $=8$ mils and $t=62$ mils. . . . . . . . 68

7.3 Insertion loss and forward transmission for an SRR on FR4 unit cell with a $=90$ mils. 69

7.4 Insertion loss and forward transmission for an SRR on FR4 unit cell with a $=150$ mils. . . . . . . . . . . . . . . . . . . . . . 69

7.5 Parametric Analysis results for square SRR on FR4 substrate with 'a' varied from 85 to 95 mils in 0.5 mil steps. ' $c$ ' $=10$ mils ' $d$ ' $=8$ mils and $t=62$ mils. . . . . . . . 69

7.6 Insertion loss and forward transmission for an SRR on FR4 unit cell with a $=88$ mils. 70

7.7 Standard S shaped SRR unit cell. . . . . . . . . . . . . . . . . 71

7.8 Parametric Analysis results for S-shaped SRR on FR4 substrate with 'w' varied from 5 to $7 \mathrm{~mm}$ in $0.1 \mathrm{~mm}$ steps. Constants: ' $h$ ' $=3 \mathrm{~mm}$ ' $c$ ' $=0.5 \mathrm{~mm}$ ' $\mathrm{a}$ ' $=10 \mathrm{~mm}$ 'b'=6.5mm 'd'=1.57mm, 'l'=2.362mm. 'd' $=8$ mils and $t=62$ mils. . . . . . . . . 72

7.9 Measured S-paramters show resonance at $8.5 \mathrm{GHz}$ when ' $w$ ' $=6.3 \mathrm{~mm} \ldots \ldots . . .73$

7.10 Final unit cell structure for the planar SRR-CLS metamaterial. The value of 'a' found through parametrics is used. . . . . . . . . . . . . . . . 74

7.11 Transmission simulation results for the SRR-CLS structure. Resonance is visible at

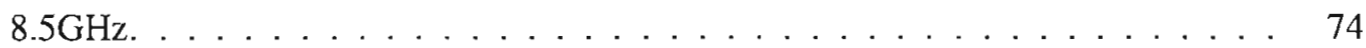

7.12 Final unit cell structure for the planar S-SRR metamaterial. . . . . . . . . . . 75 
7.13 Transmission simulation results for the S-SRR structure. Resonance is visible at

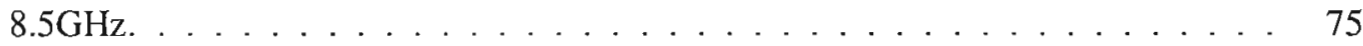

8.1 HFSS simulation setup for prism refraction setup. A waveport is used as an input. Radiation boundaries allow waves to radiate into the far-field. Top-down view does

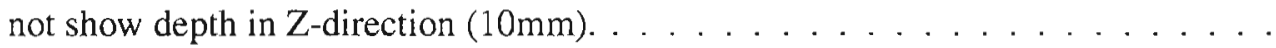

8.2 Prism outline indicating angles with respect to 0 degrees normalized to the prism normal face. . . . . . . . . . . . . . . . . . . . 78

8.3 Normalized directivity simulations for ideal prism sample for varying $\epsilon_{r}$ and $\mu_{r}$.

8.4 Normalized directivity with respect to the peak recorded value for a given frequency. Peak directivity is visible at angles negative of the prism normal for frequencies such as $7.8 \mathrm{GHz}, 8.2 \mathrm{GHz}, 8.5 \mathrm{GHz}$ and $9 \mathrm{GHz}$. The prism is operating as a normal right-hand material at $7 \mathrm{GHz}$ and $9.5 \mathrm{GHz} . \ldots \ldots . \ldots . \ldots 1$

9.1 Dimensions for Metamaterial structure containing SRR/CLS and S-SRR inclusions 83

9.2 Typical S-SRR inclusion . . . . . . . . . . . . . . . . . . . 84

9.3 Prism S-SRR structure $\ldots \ldots \ldots \ldots \ldots \ldots \ldots$

9.4 Representation of an ideal prism with the required dimensions $\ldots \ldots$. . . . . 85

9.5 Three S-SRRs in Y direction (left to right). Distance totals $1.9 \mathrm{~cm} . \quad \ldots \ldots . . . \quad 86$

10.1 Block diagram for proposed testing of DNG metamaterials in the Anechoic Chamber. 89

10.2 Sideview of testsetup structure . . . . . . . . . . . . . . . . 89

10.3 Foam board dimensions and square aperture for metamaterial slab placement. Metamaterial sample is embedded directly within an aperture in the foam board. . . . 990

10.4 Top down view of antenna chamber looking specifically at the test and positioner setup. . . . . . . . . . . . . . . . . . . . 9 90

10.5 Horn antenna placed at the minimum distance to generate a plane wave. The maximum extent of the radiation due to a beam angle of $30 \mathrm{~cm}$ is shown to be $33 \mathrm{~cm}$. . .

10.6 Reflection readings for test fixture without foam board and absorbers, with foam board/absorbers and with metal sheet in incidence path. . . . . . . . . . . 93

10.7 Transmission measurements in free space with no metamaterial slab. Plots for setup with/without foam board and absorbers. . . . . . . . . . . . . . . . . 94

10.8 Transmission and reflection with square aperture in foam board blocked. . . . . . . 94

10.9 Transmission and reflection measurements to validate test setup after normalized transmission calibration for free-space testing. . . . . . . . . . . . . 95

10.10Test setup in Anechoic chamber. Foam board has been removed to make both $\mathrm{Rx}$ and $\mathrm{Tx}$ horn antennas visibile. . . . . . . . . . . . . . . 96

11.1 Measured slab transmission data compared to theoretical data predicted by HFSS. . 99

11.2 Measured slab reflection data compared to reflection data predicted by HFSS. . . . 99

11.3 Graph showing the frequency shift in the transmission peak when changing the thickness of 31 mil spacer boards to 62 mils. . . . . . . . . . . . . . . . 100

11.4 Extracted relative permeability and permittivity for the S-shaped transmission slab. 102

11.5 Extracted index of refraction for the S-shaped transmission slab. . . . . . . . . 102

11.6 Simulated and measured $\mathrm{S} 12$ phase data. The measured data exhibits phase noise in the range from 9 to $9.8 \mathrm{GHz} \ldots \ldots \ldots \ldots . \ldots \ldots$. . . . . . . . . . . . . . . . . . . . . . . . 103

11.7 Simulated and measured $\mathrm{S} 11$ phase data. . . . . . . . . . . 103 
11.8 Simulated and measured insertion loss (S11) data for SRR/CLS metamaterial slab . 104

11.9 Simulated and measured (S12) data for SRR/CLS metamaterial slab. . . . . . . . 105

11.10Fabricated metamaterial prisms with S-SRR inclusions . . . . . . . . . . 106

11.11Theoretical diagram of prism metamaterial sample with modified dimensions. The normal angle is 26.56 degrees. All azimuthal measurements are taken with this normal angle taken as 0 degrees. . . . . . . . . . . . . . . . .

11.12Top down view diagram of modified prism. Diagram indicate reference normal angle and directions of peak radiation for 2 frequencies, one with left hand wave propagation and the other showing right hand wave propagation. . . . . . . . 108

11.13Index of refraction obtained from prism experiment. . . . . . . . . . . . . . 109

11.14 Normalized power received for two frequencies. Negative refraction is clearly visible at $8.4 \mathrm{GHz}$, while at $10 \mathrm{GHz}$, the refraction clearly exhibits standard right handed behavior. Fabricated FR-4 only metamaterial prism used to test the ability of the test setup to accurately measure the structure's index of refraction. . . . . . . . . . . . 110

11.16Index of refraction obtained from a FR-4 only prism. . . . . . . . . . . 111

11.17Normalized power received at $8.5 \mathrm{GHz}$ for the FR-4 only prism. . . . . . . . . 111

12.1 Ideal Ray diagrams showing the impact of diffraction due to a sub-wavelength aperture in 4 configurations: 1)No exit or entrance metamaterial slab, 2)Exit only slab with $k>k_{0}$. 3)Exit only slab with $k<<k_{0}$ and 4)Entrance and exit slab with $k<<k_{0} \ldots \ldots \ldots \ldots \ldots \ldots \ldots$

12.2 Top down view of simulation setup in HFSS. Parameters such as aperture size, exit slab thickness and air box size/depth may be varied with the use of variables. Default settings are aperture size $=5 \mathrm{~mm} \times 5 \mathrm{~mm}$. Slab thickness $=54 \mathrm{~mm}$ and air box depth in the $z$ direction $=10 \mathrm{~mm}$. A circle has been drawn around the setup to resemble the far field sphere set by HFSS. The goal is to maximize directivity and gain in the $\phi=90^{\circ}$ direction. . . . . . . . . . . . . . . . . . . . . . 116

12.3 Block diagram for proposed testing of DNG metamaterials in the Anechoic Chamber. 119

12.4 Sideview of testsetup structure . . . . . . . . . . . . . . . . . . . 119

12.5 Thin wire rods embedded in exit aperture slab. . . . . . . . . . . . . . . 122

12.6 Beam directivity in the azimuthal plane from a sub-wavelength aperture covered

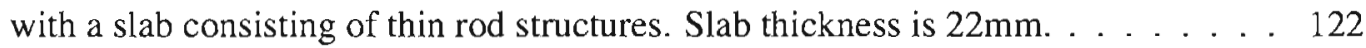

12.7 S-shaped split ring resonators embedded in exit aperture slab. . . . . . . . . . . 123

12.8 Beam directivity in the azimuthal plane from a sub-wavelength aperture covered with a slab consisting of S-shaped resonators. Slab thickness is $54 \mathrm{~mm}$. . . . . . . . 124

12.9 Gain Enhancement for a S-SRR slab placed on the exit face of a sub-wavelength aperture in comparison to the case that no slab is present. . . . . . . . . . 125

12.10 Grid mesh unit cell designed for a plasma frequency at approximately $8 \mathrm{GHz}$. Lattice paramater $\mathrm{a}=5.0 \mathrm{~mm}$ and copper strip width $\mathrm{c}=0.2 \mathrm{~mm}$. Spacing $=6 \mathrm{~mm} . \quad \ldots \ldots 126$

12.11 Transmission simulation results for grid mesh structure shown in Figure 12.12. A plasma resonance is seen at approximately $8 \mathrm{GHz}$. In this region it is expected that $\epsilon_{r}$ and $n$ approaches zero and hence $k<<1 \ldots \ldots \ldots$

12.12Grid mesh structures embedded on the exit face of the sub-wavelength aperture in HFSS. There are 3 lattice elements in the Y direction leading to a slab thickness of

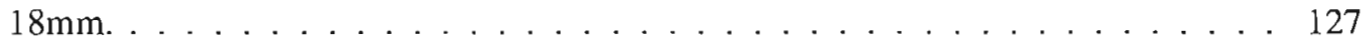


12.13Directivity when Grid mesh is placed on the exit face of the aperture. Far field plots are presented for several frequencies near the plasma resonance of the grid mesh structure. . . . . . . . . . . . . . . . . . . . . 128

12.14Gain Enhancement for a S-SRR slab placed on the exit face of a sub-wavelength aperture in comparison to the case that no slab is present. . . . . . . . . . . . . 129 


\section{List of Tables}

1.1 Sign rules of the refraction index $\ldots \ldots \ldots \ldots \ldots$

1.2 Microwave Permittivity and Permeability characteristics of homogeneous metama-

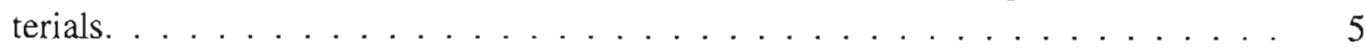

3.1 HFSS simulation results for multiple circular SRR unit cell dimensions. . . . . . . 31

4.1 Theoretical and simulated plasma frequencies for thin $3 \times 3$ wire rod structure unit cells of various wire radii and lattice spacings in an air medium. . . . . . . . .

4.2 Simulated resonant and plasma frequencies for a $2 \times 2$ array of CLS structures in air with lattice spacing, $a=2.5 \mathrm{~mm}$, height $=9.6 \mathrm{~mm}$ and microstrip width $=0.4 \mathrm{~mm}$. The capacitive load widths on each end are varied. . . . . . . . . . .

5.1 HFSS simulation results for varying unit cell dimensions. Results show the S-SRR equations provide only a very rough approximation and that HFSS simulations are absolutely necessary to characterize a SRR inclusion. . . . . . . . . . . . . 52

5.2 HFSS simulation results for varying loop ratios to alter the two resonance frequencies. 55

5.3 Summary of the transmission, permittivity and permeability characteristics of the inclusions introduced in this thesis. . . . . . . . . . . . . . 56

7.1 Square SRR parameters . . . . . . . . . . . . . . . . . . 67

7.2 Comparison of various transmission test results for a SRR-CLS unit cell where vertical electrical spacing between the CLS and SRR inclusions is varied. Measurements taken at the resonance frequency $f_{0} \ldots \ldots \ldots \ldots \ldots \ldots$

7.3 S-SRR parameters . . . . . . . . . . . . . . . . . . . 72

8.1 Table showing agreement between theoretical calculations for $n$ using $n=\sqrt{\epsilon_{r} \mu_{r}}$ and simulated results. Agreement is accurate in regions where $|n| \geq 1 \ldots \ldots$

8.2 Table showing frequency dependent index of refraction obtained from far field radiation pattern simulations of S-shaped SRR metamaterial prism structure in HFSS.

12.1 Table shows relationship between exit face metamaterial $\mu_{r}, \epsilon_{r}$ and directivity. As permeability and permittivity is reduced, $k<0$ and the directivity is enhanced. . .

12.2 Table shows relationship between aperture width, directivity and Gain. HFSS simulation results at $8.5 \mathrm{GHz}$ are obtained using a homogeneous ideal slab with zero loss tangent $\delta, \epsilon_{r}=0.1$ and $\mu_{r}=0.1$. The slab thickness was held constant at $54 \mathrm{~mm} \quad . \quad 120$

B.1 Bill of materials for products ordered from Current Composites. . . . . . . . . . 143 
B.2 Bill of materials for products ordered from the PCB company. . . . . . . . . . 143 


\section{Chapter 1}

\section{Introduction}

\subsection{Metamaterials}

Metamaterials are inherently artificial materials, not found in nature and which yield interesting electromagnetic responses. Jagadis Chock in 1898 constructed the first metamaterial out of artificial chiral elements [1]. Lindman, in 1914 made artificial chiral media by embedding many randomly oriented small wire helices in the host medium [2]. Kock in 1948 [3] created microwave lenses by embedding metallic strips, wires and disks periodically in order to tailor the artificial media's refractive index. As fabrication methods improve, the inclusions (artificial structures) embedded in the host medium reduce in size.

Electromagnetic waves interact with inclusions in the host medium, induce electric and magnetic moments, which in turn affect the material's transmission capabilities and material parameters such as permeability and permittivity. When characterizing the permittivity $(\epsilon)$ and permeability $(\mu)$ of a metamaterial, one must characterize the structure as homogeneous. If one considers a single inclusion as part of a unit cell periodically embedded within the host medium, its size $p$ must be less than a quarter of the incident radiation wavelength: $p<\frac{\lambda}{4}$. This homogeneity relation is a rule of thumb condition. The relation is commonly used in distinguishing lumped components from

quasi-lumped components, $\left(\frac{\lambda}{4}<p<\frac{\lambda}{2}\right)$ and distributed components: $\left(p>\frac{\lambda}{2}\right)$. This condition ensures that refractive phenomena inherent in homogeneous materials dominate over scattering and 
diffusion effects. The electromagnetic radiation is essentially unaware of the lattice structure in the host medium and maintains field uniformity in the direction of propagation within the structure.

\subsubsection{Basic plane wave theory}

A plane wave can be described in terms of three vectors: Electromagnetic field vectors $\vec{E}$ and $\vec{H}$, as well as the wave number vector in the direction of propagation, $\vec{k}$. Mathematically, the plane wave may be represented as:

$$
\begin{aligned}
& E(r)=E_{0} e^{j(\vec{k} \vec{r}-\omega t)} \\
& H(r)=H_{0} e^{j(\vec{k} \vec{r}-\omega t)}
\end{aligned}
$$

In a linear, homogeneous, isotropic, dispersionless medium such as vacuum, there is no free charge or current present and hence Maxwell's Equations of free space are:

$$
\begin{aligned}
\nabla \cdot \vec{E} & =0 \\
\nabla \cdot \vec{B} & =0 \\
\nabla \times \vec{E} & =-\frac{\partial \vec{B}}{\partial t} \\
\nabla \times \vec{B} & =\mu_{0} \epsilon_{0} \frac{\partial \vec{E}}{\partial t}
\end{aligned}
$$

The above relations for Maxwell's equations in differential form can be represented in the Gaussian system. Defining the electric displacement field $D=\epsilon E$ and $B=\mu H$, the Maxwell Equations can be stated as:

$$
\begin{aligned}
\nabla \times \vec{E} & =-\frac{\partial \vec{B}}{\partial t}=-\frac{\mu_{r} \mu_{0}}{c} \frac{\partial \vec{H}}{\partial t} \\
\nabla \times \frac{\vec{B}}{\mu_{0}} & =\frac{\epsilon_{r} \epsilon_{0}}{c} \frac{\partial \vec{E}}{\partial t}
\end{aligned}
$$

Mathematical equations for a plane wave may be substituted into the above Gaussian system 
Maxwell Equations to yield:

$$
\begin{aligned}
\vec{k} \times \vec{E} & =\omega \mu \vec{H} \\
\vec{k} \times \vec{H} & =-\frac{1}{\omega} \mu \vec{E}
\end{aligned}
$$

Thus, the curl of $\vec{E}$ and $\vec{H}$ is proportional to the cross product of the $\vec{k}$ vector and the $\vec{E}$ or $\vec{H}$ field. These vectors follow the right hand rule. Power flow is described by the Poynting Vector:

$$
\vec{S}=\vec{E} \times \vec{H}
$$

In the usual case of plane wave propagation where the right-hand rule may be applied to a wave with component $\vec{k}$ parallel to the direction of power flow, $\epsilon$ and $\mu$ are positive. Finally, a positive refraction index may then be realized using the standard relation:

$$
n=\sqrt{\epsilon_{r} \cdot \mu_{r}}
$$

\subsection{Double Negative Index metamaterials}

In 1967, physicist V.G. Veselago from the Lebedjev Physical institute of Moscow theoretically investigated plane wave propagation in a material whose permittivity and permeability were assumed negative [4]. With both parameters negative, equation (1.4) shows that the propagation vector $\vec{k}$ reverses direction and the set of three vectors: $\vec{k}, \vec{E}, \vec{H}$ follow a left-hand rule. Hence double negative materials are also known as Left-handed materials. As the $\vec{k}$ vector has reversed direction, the phase velocity is now opposite to power flow (denoted by the Poynting vector). The refractive index of the material must also be considered. The square root in (1.6) is not violated if both permeability and permittivity are negative. However, refraction is also phase sensitive [4], thus $n$ becomes neg- . ative. A separate argument may be made for the sign choice in the index of refraction calculation. In [7], it is pointed out that one cannot assume $n<0$ for a double negative index metamaterial. 
However, the constraint that the source must do positive work on the external fields may be made. With this constraint in mind, a current sheet radiating into a left-handed medium may be analyzed. A current sheet is depicted in Figure 1.1. The density of the current sheet polarized in the $z$ direction is:

$$
\mathbf{J}=\hat{z} \mathbf{J}_{o} e^{-j \beta x} \delta(x)
$$

where $J_{0}$ is the strength of the surface current density, $\beta$ is the rate of linear phase change in the $x$ direction. The direction of current flow is assumed to be $z$. The direction of phase variation is along $x$. Note the anti-parallel nature of the wave vector $k$ and the Poynting vector $S$. Using $\Omega$ as the oscillation frequency of the source current, [7] derives the current sheet power as:

$$
P=\Omega W=-\frac{1}{2} \int_{v} j^{*} E(x, \Omega) d x=\pi \frac{\mu}{c n} j_{0}^{2}
$$

From (1.8), positive power is needed for positive work to be done by the source on the surrounding fields. In the case when $\mu<0$, the index of refraction, $\mathrm{n}$ must also be less than zero $(n<0)$. For radiation in a right-handed medium where $\mu>0$, the index of refraction $\mathrm{n}$, must also be greater than zero $(n>0)$.

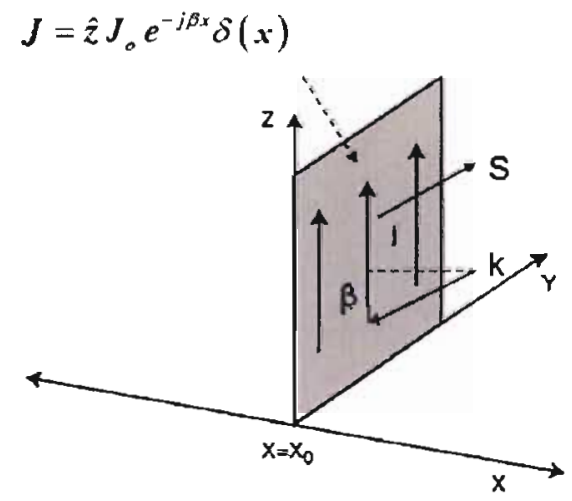

Figure 1.1 Current sheet at $x=x_{0}$ radiates into a left handed medium Source: D.R.Smith, Duke University. 
Table 1.1 depicts the sign rules for material refractive index while Table 1.2 introduces some basic properties and names for materials for various combinations of permeability and permittivity.

\begin{tabular}{||c|c|r||}
\hline & $\epsilon_{r}>0$ & $\epsilon_{r}<0$ \\
\hline$\mu_{r}>0$ & positive & imaginary \\
\hline$\mu_{r}<0$ & imaginary & negative \\
\hline
\end{tabular}

Table 1.1 Sign rules of the refraction index

\begin{tabular}{||c|c|c|c||}
\hline$\epsilon>0, \mu>0$ & $\epsilon<0, \mu>0$ & $\epsilon>0, \mu<0$ & $\epsilon<0, \mu<0$ \\
\hline $\begin{array}{c}\text { - Forward wave } \\
\text { propagation }\end{array}$ & $\begin{array}{c}\text { - Metal like } \\
\text { characteristics at }\end{array}$ & $\begin{array}{c}\text { - Ferrimagnetic material } \\
\text { characteristics }\end{array}$ & $\begin{array}{c}\text { - Left-handed medium } \\
\text { - Backward-wave } \\
\end{array}$ \\
& optical frequencies & & propagation \\
& - Evanescent waves & - Evanescent waves & - $\operatorname{Re}(\mathrm{n})<0$ \\
\hline
\end{tabular}

Table 1.2 Microwave Permittivity and Permeability characteristics of homogeneous metamaterials.

Snell's Law is commonly used to describe the relationship between angles of incidence and refraction, when referring to light or electromagnetic waves passing through a planar boundary between two passive isotropic media. If $n_{1}$ and $\theta_{1}$ describe the refractive index and incidence angle of the incoming wave in the first medium, while $n_{2}$ and $\theta_{2}$ refer to the second medium, Snell's law is represented by:

$$
n_{1} \sin \theta_{1}=n_{2} \sin \theta_{2}
$$

If $n_{1}$ is negative, then:

$$
\begin{aligned}
\theta_{2} & =\sin ^{-1}\left(\frac{n_{1} \sin \theta_{1}}{n_{2}}\right) \\
& =\sin ^{-1}(\xi<0) \\
\ldots & \\
\theta_{2} & <0^{\circ}
\end{aligned}
$$


where $\xi$ substitutes for the argument of the arcsine function. Note that the angle of incidence with respect to the normal is presumed to be $0^{\circ}<\theta_{1}<90^{\circ}$. Refer to Figure 1.2.

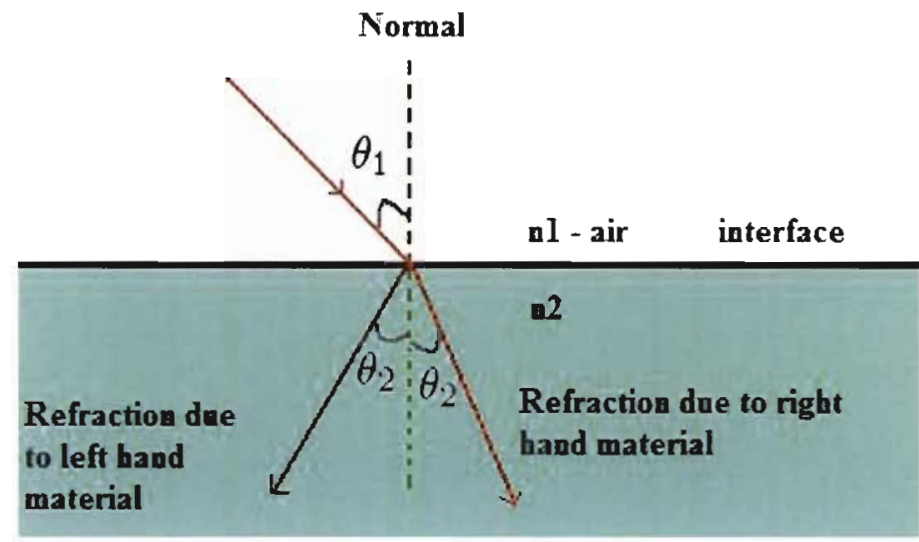

Figure 1.2 Depiction of refraction according to Snell's law. Negative refractive materials show refraction on the other side of the normal. Snell's law is clearly preserved but the angle for $\theta_{2}$ is negative.

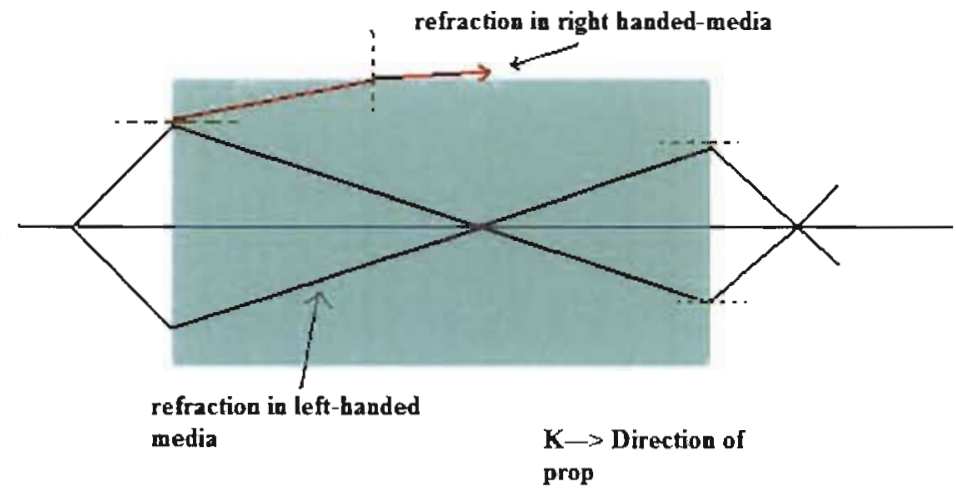

Figure 1.3 Refraction in a slab of left-handed/Double negative material. The negative refractive index causes the bending of rays to negative angles of the surface normal. The diverging beams of a point source convege back towards a focal point. The first focal point may form inside the slab if the material has sufficient thickness. 


\section{Backward wave propagation simulation}

The group velocity $V_{g}$ and phase velocity $V_{p}$ must be considered when looking at the backward wave propagation phenomenon of negative index metamaterials. The group velocity indicates velocity at which the wave envelope or wave amplitude modulates. It best describes the rate at which information is conveyed along a wave. The phase velocity of a wave is the velocity at which the phase of one frequency component of the wave travels. The group velocity indicates the veloctiy of the collective wave or wave packet while the phase velocity indicates the velocity of the wave nodes i.e crests. Velocity is a vector, hence, has both a magnitude and direction associated with it. The Poynting vector as previously described denotes the group velocity and is anti-parallel to the phase velocity in a left-handed (double-negative) medium. A simulation is conducted in HFSS to show this phenomenon (see Figure 1.4) in a parallel plate waveguide configuration. The top and bottom transparent faces are defined as perfect E planes to approximate a parallel plate setup. Both ends have waveports to introduce the radiation. The waveguide is divided into 3 sections. The first and last sections are a free space material with $\epsilon_{r}=+1, \mu_{r}=+1$. The middle material is defined with bulk parameters $\epsilon_{r}=-1, \mu_{r}=-1$.

\section{Lens simulation}

HFSS is used to simulate the lens focusing characteristics of double negative media. Two slabs are placed adjacent to each other. A cylindrical source with propagation vector $I$ polarized in the $\mathrm{Z}$ direction is situated within the right handed medium $\left(\epsilon_{r}=4.4, \mu_{r}=1\right)$ while radiation boundaries are placed around the left handed medium with bulk parameters: $\epsilon_{r}=-1, \mu_{r}=-1$. Simulation occurs at $8.5 \mathrm{GHz}$. As seen in Figure 1.5, a focal point or image is seen within the left-handed medium. 


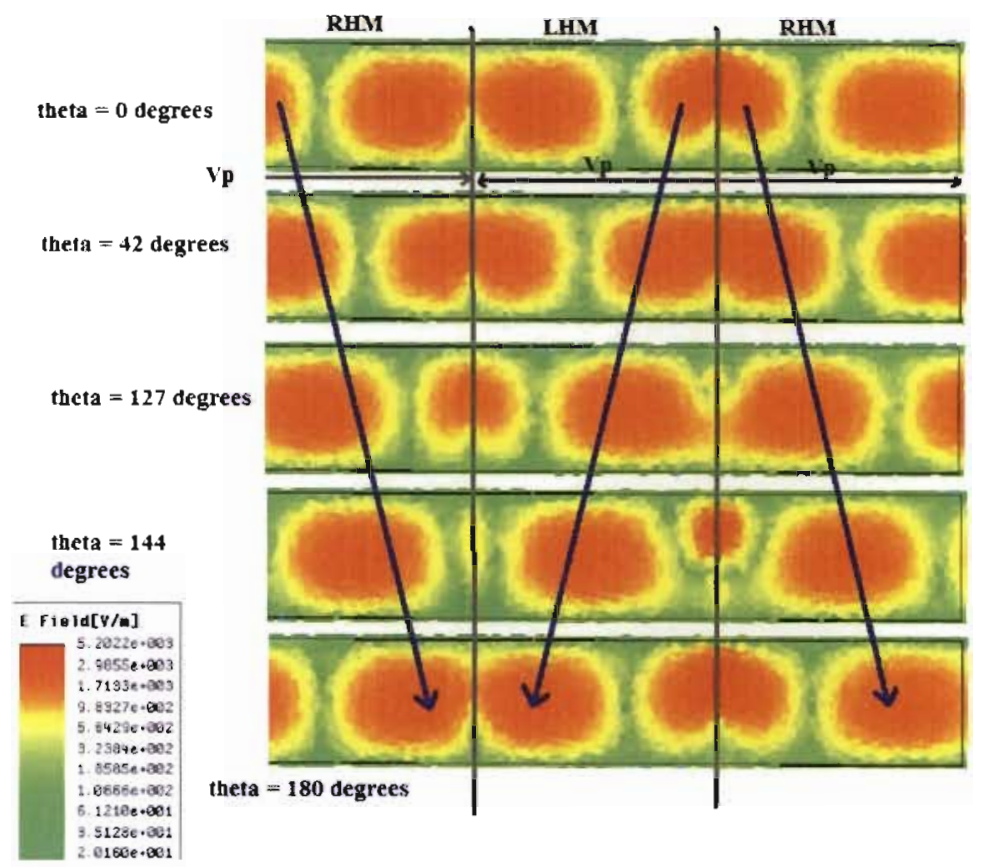

Figure 1.4 Simulation in Ansoft HFSS of a parallel plate waveguide. The direction of phase velocity is shown with arrows. It can be seen in increasing the phase from $0^{0}$ to $180^{\circ}$ that the phase velocity is opposite in the middle material is opposite to the materials on either end.

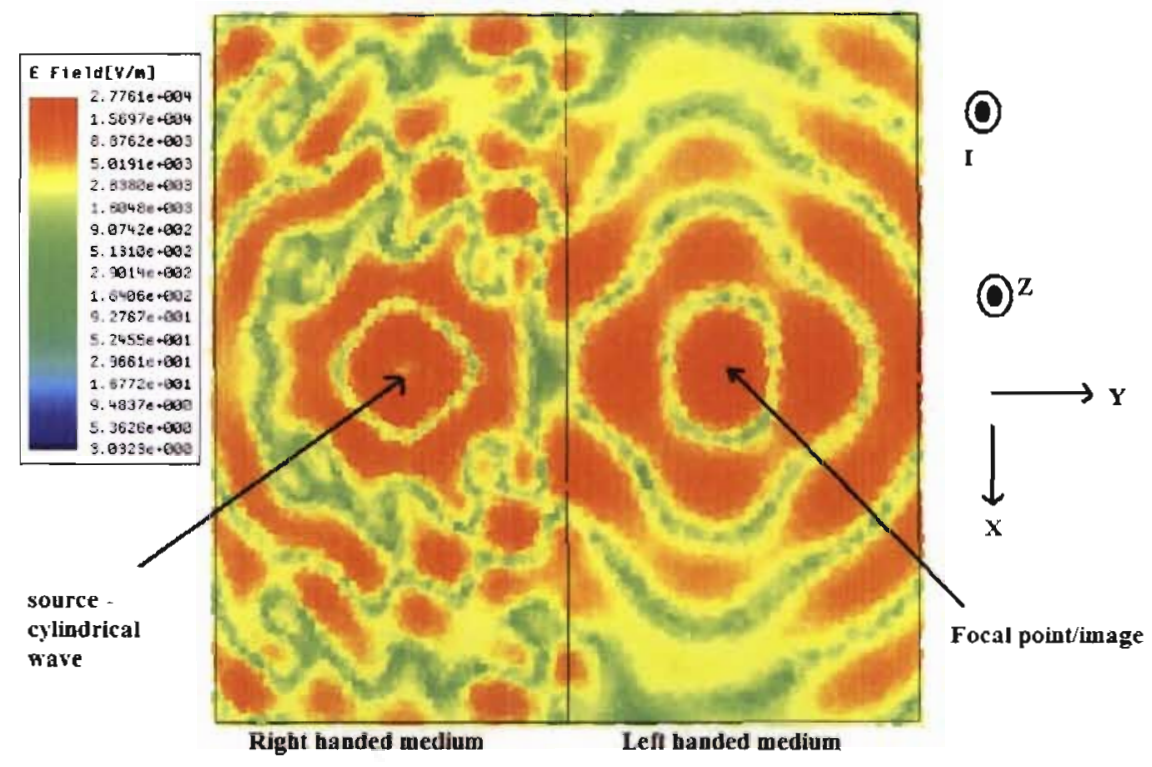

Figure 1.5 Simulation in Ansoft HFSS of the lens/negative refractive attributes of double negative/left handed media. 


\section{Chapter 2}

\section{Parameter Extraction}

\subsection{Nicolson-Ross-Weir method}

The equations published by Nicolson, Ross and Weir [12] enable the calculation of the complex permittivity and permeability of a material sample (Figure 2.1) from the measured S-parameters. The correlation between S-parameters and material properties is derived here by considering multiple reflections of a unit amplitude wave incident upon the air-sample interfaces within the waveguide. The multiple reflections at the air-sample interfaces within a filled waveguide are shown below:

When a wave is incident upon the air-sample interface, partial reflection and transmission occurs. The partial reflection is accounted for by and the transmitted portion. Partial reflection and transmission occurs again when the wave strikes the sample-air interface. Summing the reflections occurring at the first sample interface yields:

$$
\begin{aligned}
\Gamma_{i n} & =\Gamma_{1}+T_{12} T_{21} \Gamma_{3} e^{-j 2 \gamma d}+T_{12} T_{21} \Gamma_{3}^{2} \Gamma_{2} e^{-j 4 \gamma d}+\ldots \\
& =\Gamma_{1}+T_{12} T_{21} \Gamma_{3} e^{-j 2 \phi} \sum_{n=0}^{\infty} \Gamma_{2}^{n} \Gamma_{3}^{n} e^{-2 j n \theta}
\end{aligned}
$$

Using the identity $\sum_{n=0}^{\infty} r^{n}=\frac{1}{1-r}$, where $r=\Gamma_{2} \Gamma_{3} e^{-j 2 \theta}$ :

$$
\Gamma_{i n}=\Gamma_{1}+\frac{T_{12} T_{21} \Gamma_{3} e^{-j 2 \theta}}{1-\Gamma_{2} \Gamma_{3} e^{-j 2 \theta}}
$$




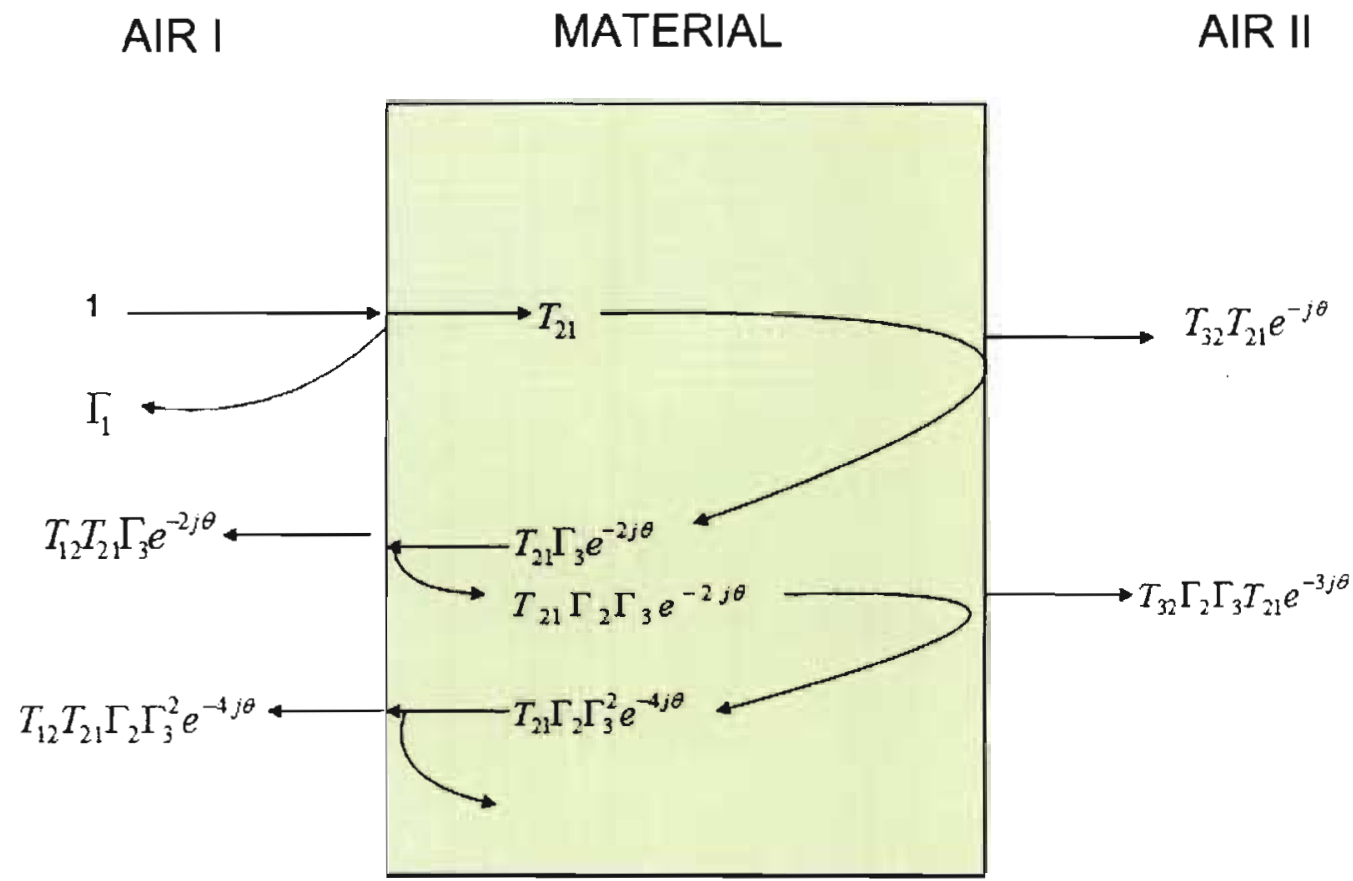

Figure 2.1 A filled waveguide undergoing excitation encounters multiple reflections at the air-sample interfaces.

One can obtain the $S$ parameter equivalent for $\Gamma_{i n}$ and $S_{11}$ through some manipulation. Let $\Gamma_{2}=\Gamma_{3}=-\Gamma_{1}$. Then:

$$
\begin{aligned}
\Gamma_{1} & =\frac{Z-1}{Z+1} \\
T_{12} & =1+\Gamma_{2} \\
T_{21} & =1+\Gamma_{1} \\
S_{11} & \equiv \Gamma_{\text {in }}=\frac{\Gamma_{1}\left(1-z^{2}\right)}{1-\Gamma_{1}^{2} z^{2}}
\end{aligned}
$$

Note that the relations $z^{2}=e^{-j 2 \theta}$ and $\theta=\gamma d$ are used in (2.3). The lower-case $\mathrm{z}$ in this case is the propagation factor through the sample. The expression for $S_{11}$ is then found by substituting the terms in (2.3) into (2.2). Next, the sum of all wave components transmitted completely through the 
material sample may be obtained:

$$
\begin{aligned}
T_{\text {out }} & =T_{32} T_{21} e^{-j \theta} \sum_{n=0}^{\theta} \Gamma_{2}^{n} \Gamma_{3}^{n} e^{j(2 n+1) \theta} \\
\text { Total Transmitted } & =\frac{T_{32} T_{21} e^{-j \theta}}{1-\Gamma_{2} \Gamma_{3} e^{-j 2 \theta}} \\
\text { Letting } T_{32} & =1+\Gamma_{2}, S_{21} \equiv \frac{z\left(1-\Gamma_{1}^{2}\right)}{1-\Gamma_{1}^{2} z^{2}}
\end{aligned}
$$

The values of $z$ (complex transmission constant, also referenced as $T$ in some papers) and $\Gamma_{1}$ remain unknown at this point. Sum and difference properties of S-parameters are implemented as follows:

$$
\begin{aligned}
& V_{1}=S_{21}+S_{11} \\
& V_{2}=S_{21}-S_{11}
\end{aligned}
$$

The product of $V_{1}$ and $V_{2}$ is therefore:

$$
\begin{aligned}
V_{1} V_{2} & =\left(S_{21}+S_{11}\right)\left(S_{21}-S_{11}\right) \\
& =S_{21}^{2}-S_{11}^{2} \\
& =\left[\frac{z\left(1-\Gamma_{1}^{2}\right)}{1-\Gamma_{1}^{2} z^{2}}\right]^{2}-\left[\frac{\Gamma_{1}\left(1-z^{2}\right)}{1-\Gamma_{1}^{2} z^{2}}\right]^{2} \\
& =\frac{z^{2}\left(1-\Gamma_{1}^{2}\right)^{2}-\Gamma_{1}^{2}\left(1-z^{2}\right)^{2}}{1-\Gamma_{1}^{2} z^{2}} \\
& =\frac{z^{2}-2 z^{2} \Gamma_{1}^{2}+z^{2} \Gamma_{1}^{4}-\Gamma_{1}^{2}+2 z^{2} \Gamma_{1}^{2}-z^{4} \Gamma_{1}^{2}}{1-\Gamma_{1}^{2} z^{2}} \\
& =\frac{z^{2}-\Gamma_{1}^{2}}{1-\Gamma_{1}^{2} z^{2}}
\end{aligned}
$$

The difference of $V_{2}$ and $V_{1}$ is:

$$
\begin{aligned}
V_{1}-V_{2} & =S_{21}+S_{11}-S_{21}+S_{11} \\
& =2 S_{11} \\
& =\frac{2 \Gamma_{1}\left(1-z^{2}\right)}{1-\Gamma_{1}^{2} z^{2}}
\end{aligned}
$$


Let $X=\frac{1-V_{1} V_{2}}{V_{1}-V_{2}}$, and using (2.7) and (2.8) one can find $X$ in terms of $\Gamma_{1}$ :

$$
X=\frac{1+\Gamma_{1}^{2}}{2 \Gamma_{1}}
$$

Now $\Gamma_{1}$ may be determined in terms of $X$ using the quadratic formula $\frac{-b \pm \sqrt{b^{2}-4 a c}}{2 a}$ :

$$
\begin{aligned}
2 \Gamma_{1} X & =1+\Gamma_{1}^{2} \\
\Gamma_{1}^{2}-2 \Gamma_{1} X+1 & =0 \\
\Gamma_{1} & =X \pm \sqrt{X^{2}-1}
\end{aligned}
$$

The choice of sign in (2.10) should yield $\left|\Gamma_{1}\right| \leq 1$. Using $\Gamma_{1}, S_{11}$, and $S_{21}$, one can determine the value of the complex exponential $z$.

$$
\begin{gathered}
V_{1}-\Gamma_{1}=S_{21}+S_{11}-\Gamma_{1} \\
=\frac{\Gamma_{1}\left(1-z^{2}\right)}{1-\Gamma_{1}^{2} z^{2}}+\frac{z\left(1-\Gamma_{1}^{2}\right)}{1-\Gamma_{1}^{2} z^{2}}-\Gamma_{1} \\
=\frac{\Gamma_{1}-\Gamma_{1}^{2} z^{2}+z-\Gamma_{1}^{2} z-\Gamma_{1}+\Gamma_{1}^{3} z^{2}}{1-\Gamma_{1}^{2} z^{2}} \\
1-V_{1} \Gamma_{1}=\frac{1-z \Gamma_{1}+z \Gamma_{1}^{3}-\Gamma_{1}^{2}}{1-\Gamma_{1}^{2} z^{2}} \\
\frac{V_{1}-\Gamma_{1}}{1-V_{1} \Gamma_{1}}=\frac{\left(1-\Gamma_{1}^{2}\right)\left(z-z^{2} \Gamma_{1}^{2}\right)}{1-\Gamma_{1}^{2} z^{2}} \cdot \frac{1-\Gamma_{1}^{2} z^{2}}{\left(1-\Gamma_{1}^{2}\right)-z \Gamma_{1}\left(1-\Gamma_{1}^{2}\right)-z \Gamma_{1}\left(1-\Gamma_{1}^{2}\right)} \\
=\frac{z\left(1-z \Gamma_{1}\right)}{1-z \Gamma_{1}} \\
=z \quad
\end{gathered}
$$

\subsubsection{Complex propagation constant of the material under test}

The complex exponential transmission term $z$ relates to the propagation constant of the material. Using the $z$ term, one may calculate the complex propagation constant $(\gamma)$ of the material. The method used is known as de Moivre's Theorem and may be represented as: 


$$
(\cos x+j \sin x)^{n}=\cos (n x)+j \sin (n x)
$$

Noting as explained earlier, that $z^{2}=e^{-j 2 \theta}$ where $\theta=\gamma d$, the complex propagation constant may be found as follows:

$$
\begin{aligned}
z^{n} & =e^{-j n \theta} \\
& =\cos (n \theta)+j \sin (n \theta) \\
& =(\cos (\theta)+j \sin (\theta))^{n} \\
\frac{1}{z^{n}} & =(\cos (\theta)+j \sin (\theta))^{-n} \\
\frac{1}{z} & =\cos \theta+j \sin \theta=e^{\gamma d} \\
\ln \left(\frac{1}{z}\right) & =\gamma d \\
\frac{1}{d} \ln \left(\frac{1}{z}\right) & =\gamma
\end{aligned}
$$

The variable $d$ in (2.15) is the sample thickness. Knowledge of the sample thickness, complex propagation constant and characteristic impedance of a filled waveguide allow one to determine the complex permittivity and permeability of a material. However, exact expressions for the permeability and permittivity of these materials depend on the fundamental propagation mode in the waveguide and the sample's intrinsic impedance. A rectangular waveguide and coaxial line are discussed here. The coaxial line was used by Nicolson and Ross to verify the NRW method to obtain the intrinsic properties of a material. In the next section, a discussion of the NRW method as pertaining to double negative index metamaterials is analyzed.

\subsubsection{Intrinsic parameters for a Coaxial Waveguide}

In a lossless coaxial waveguide, the fundamental mode of propagation is the TEM mode. The normalized impedance of the filled line section is the same as that of an infinitely long material in 
freespace.

$$
\begin{gathered}
Z \equiv Z_{\text {infinite d }}=\sqrt{\frac{\mu_{r}}{\epsilon_{r}}} \\
\gamma=j \frac{2 \pi}{\lambda_{0}} \sqrt{\epsilon_{r} \mu_{r}} \\
\text { Refractive index: } N=\sqrt{\epsilon_{r} \mu_{r}} \\
=-j \frac{\gamma \lambda_{0}}{2 \pi}
\end{gathered}
$$

The material's complex permittivity and permeability are calculated by combining (2.16), (2.17) and (2.18):

$$
\begin{aligned}
& \mu_{r}=N \cdot Z \\
& \epsilon_{r}=\frac{N}{Z}
\end{aligned}
$$

\subsubsection{Intrinsic parameters for a Rectangular Waveguide}

In a rectangular waveguide, the guided wavelength differs from the free space value according to the equation [33]:

$$
\lambda_{g}=\frac{\lambda_{0}}{\sqrt{1-\left(\frac{\lambda_{0}}{\lambda_{c}}\right)^{2}}}
$$

The cutoff wavelength is denoted as $\lambda_{c}$ while the guided wavelength is $\lambda_{g}$. In the $T E_{10}$ mode, the cutoff wavelength equals twice the longest transverse dimension of the waveguide. Equations 
for the waveguide are shown below for air filled waveguide and sample filled waveguide:

$$
\begin{aligned}
Z_{\text {air }} & =\sqrt{\frac{\mu_{0}}{\epsilon_{0}}} \frac{\lambda_{g}}{\lambda_{0}} \\
Z_{\text {sample }} & =j \frac{2 \pi f \mu_{r} \mu_{0}}{\gamma_{\text {guide }}} \\
\gamma_{\text {guide }} & =\gamma^{2}+\left(\frac{2 \pi}{\lambda_{c}}\right)^{2}
\end{aligned}
$$

The normalized characteristic impedance $Z$ may be found using the air filled and sampled filled characteristic impedances. This normalized characteristic impedance is the capitalized $Z$ present in (2.3).

$$
Z=\frac{Z_{\text {sample }}}{Z_{\text {air }}}=j \frac{2 \pi f \mu_{r} \mu_{0}}{\gamma_{\text {guide }}} \cdot \frac{\lambda_{0}}{\lambda_{g}} \sqrt{\frac{\epsilon_{0}}{\mu_{0}}}
$$

Now one may solve for the relative permeability and permittivity of the filled rectangular waveguide:

$$
\begin{aligned}
\mu_{r} & =\frac{\gamma_{\text {guide }} Z \frac{\lambda_{g}}{\lambda_{0}}}{j \frac{2 \pi}{\lambda_{0}}} \\
N & =\frac{1}{j \frac{2 \pi}{\lambda_{0}}} \sqrt{\gamma_{\text {guide }}^{2}-\left(\frac{2 \pi}{\lambda_{c}}\right)^{2}} \\
\epsilon_{r} & =\frac{N^{2}}{\mu_{r}}
\end{aligned}
$$

\subsection{An implementation of the NRW method pertaining to Double Neg- ative Metamaterials}

In [10], Ziolkowski briefly introduces the NRW method, but explains that the original form of the analysis is unsuitable for calculating permittivites and permeabilities of DNG materials. The standard extraction expressions are unsatisfactory in the frequency regions where the permittivity and permeability resonances were expected. At these frequencies, a sharp transition between positive and negative values is expected. The presence of square root values in (2.10) makes calculations difficult in regions of permittivity and permeability resonance. While the sign preceding the square 
root operation must yield $\left|\Gamma_{1}\right| \leq 1$, the choice is difficult when S-parameters resonate and the choice of sign may potentially bias the end result.

Using the steps outlined after (2.12), the following relations can be found:

$$
\begin{aligned}
z & =\frac{V_{1}-\Gamma_{1}}{1-V_{1} \Gamma_{1}} \\
\text { similarly, } \Gamma_{1} & =\frac{z-V_{2}}{1-z V_{2}}
\end{aligned}
$$

Note that $z$ is a complex exponential transmission term. From the above expressions, the following may be obtained:

$$
\begin{aligned}
1-z & =\frac{\left(1-V_{1}\right)\left(1+\Gamma_{1}\right)}{1-\Gamma_{1} V_{1}} \\
\eta & =\frac{1+\Gamma_{1}}{1-\Gamma_{1}} \\
& =\frac{1+z}{1-z} \cdot \frac{1-V_{2}}{1+V_{2}}
\end{aligned}
$$

Note that for finite slab thicknesses, the transmission coefficient between two faces of the slab: $z=e^{-j \omega \sqrt{\mu \epsilon} d}=e^{-j k d}[12]$. To simplify the analysis, one may take the Taylor expansion of this exponential function and obtain:

$$
\text { Taylor series definition : } \begin{aligned}
& \sum_{n=0}^{\infty} \frac{f^{(n)}(a)}{n !}(x-a)^{n} \text { where } \mathrm{a}=0 \text { in this case } \\
z & \approx 1-j k d e^{-j k d}-k^{2} d^{2} \frac{e^{-j k d}}{2} \ldots \\
z & \approx 1-j k d-\frac{k^{2} d^{2}}{2} .
\end{aligned}
$$

For the simplification to be valid and the higher order functions of the Taylor series to be negligible, the product of the real part of the wave vector and the slab thickness must be less than unity. Hence the slab must be "thin": $\operatorname{Re}(k) \cdot d<1$. Therefore, the complex transmission term $z$ may be approximated as:

$$
z \approx 1-j k d
$$


The wave vector is calculated for each measurement frequency using the standard relation: $k=$

$\omega \frac{\sqrt{\epsilon_{r} \mu_{r}}}{c}=k_{0} \sqrt{\epsilon_{r} \mu_{r}}$. Using this approximation, expressions for the wave vector $k$ and permeability $\mu$ may be obtained from (2.26). Expressions for $V_{1}$ and $V_{2}$ were developed earlier in terms of the scattering parameters.

$$
\begin{aligned}
k & \approx \frac{1}{j d} \frac{\left(1-V_{1}\right)\left(1+\Gamma_{1}\right)}{1-\Gamma_{1} V_{1}} \\
\mu_{r} & \approx \frac{2}{j k_{0} d} \frac{1-V_{2}}{1+V_{2}}
\end{aligned}
$$

The permittivity and index of refraction may be obtained:

$$
\begin{aligned}
\epsilon_{r} & =\left(\frac{k}{k_{0}}\right)^{2} \frac{1}{\mu_{r}} \\
n & =\sqrt{\epsilon_{r} \mu_{r}}=\sqrt{\left(\frac{k}{k_{0}}\right)^{2} \frac{1}{\mu_{r}} \cdot \mu_{r}} \\
& =\frac{k}{k_{0}}
\end{aligned}
$$

An alternative equation for the relative permittivity $\epsilon_{r}$ may be obtained by finding the square of the wave impedance:

$$
\eta^{2}=\frac{\mu_{r}}{\epsilon_{r}}=\frac{1+V_{1}}{1-V_{1}} \frac{1-V_{2}}{1+V_{2}}=\frac{\left(S_{11}+1\right)^{2}-S_{21}^{2}}{\left(S_{11}-1\right)^{2}-S_{21}^{2}}
$$

While the above relation for permittivity is valid, it will be shown in Section 6.1 that resonance features in permittivity are not as visible compared to the relation for permittivity presented by R.W Ziolkowski (2.31) [10]. In the case of (2.29), results may not show a resonance as predicted by the S-parameters [10]. Ziolkowski takes another approach to finding the relative permittivity of a metamaterial slab. The relation for $\mathrm{S} 11$ for a slab of finite thickness $d$ where the "thin" constraint 
$\operatorname{real}(k) \cdot d<1$ holds:

$$
S_{11}=\frac{\left(\eta^{2}-1\right)\left(1-z^{2}\right)}{(\eta+1)^{2}-(\eta-1)^{2} z^{2}}
$$

Where $z=e^{-j k d}$

$$
S_{11} \approx \frac{2 j k d\left(\eta^{2}-1\right)}{(\eta+1)^{2}-(\eta-1)^{2}}=2 j k d \frac{\eta^{2}-1}{4 \eta}
$$

solving for epsilon yields

$$
\epsilon_{r} \approx \mu_{r}+j \frac{2 S_{11}}{k_{0} d}
$$

The above relation clearly shows that relative permittivity tracks the material's relative permeability. Note that as $S_{11}$ goes to zero the value of the relative permeability dominates. This is to be expected in double negative applications where the $\left|S_{11}\right|$ parameter approaches zero while peak transmission occurs in the frequency range of interest. However, the latter term must be large enough to support the case where only permeability goes negative (i.e: SRR only media). Such a metamaterial would require parameter extraction using a different set of relations.

\subsection{Divergence in the Nicolson-Ross-Weir equations}

It has been shown that the permittivity and permeability of a sample are uniquely related to the reflection and transmission co-efficients. However it has been noted by several sources [14] [15] that uncertainty is introduced into the numerical analysis when sample thicknesses approach a $\frac{1}{2}$ multiple of the guided wavelength $\lambda$. Consider the case where the sample thickness $d$, is related

to the wavelength in the sample under test $\lambda_{g}$ by $d=\frac{n \cdot \lambda_{g}}{2}$. In this case, the transmission constant $z$ ( $T$ in some papers) approaches \pm 1 . Hence, $z=e^{j k d} \rightarrow \pm 1$. Examining equation (2.8) which relates $V_{2}-V_{1}$ to $S_{11}$, it is seen that the numerator and hence $S_{11}$ approaches zero. A divergence or pole is seen in the variable $X$ presented earlier. When $S_{11}$ approaches zero mainly due to the influence of the structure thickness, steps must be taken to ensure that the electrical thickness does not approach $\lambda / 2$ in the frequency range of interest. One solution is to use thin samples smaller than the illuminating frequency's wavelength. While one may not expect the algorithm to be stable over 
a broad range of input frequencies, for the case of this project, the resonance frequency is restricted to the X-band region; specifically $8.5 \mathrm{GHz}$. Hence, at $8.5 \mathrm{GHz}$ the divergence problem in the NRW method should be avoided. For this project as will be shown in chapter 9 , a slab thickness of $2.6 \mathrm{~cm}$ was chosen. This is approximately $0.75 \lambda$ at $8.5 \mathrm{GHz}$ and the maximum distance away from a $\lambda / 2$ multiple. 


\section{Chapter 3}

\section{The Split Ring Resonator Inclusion}

It is important to consider the metallic inclusions needed to obtain negative permittivity and permeability using periodically structured metamaterials. This chapter specifically deals with an inclusion that responds to magnetic fields and hence has the potential to yield negative permeability.

\subsection{Split Ring Resonators and Permeablility}

J.B Pendry [17] hypothesizes that for periodic structures defined by a unit cell of characteristic dimension $p$, a condition for the response of the system to electromagnetic excitation is as follows:

$$
p<\lambda=\frac{2 \pi c_{0}}{\omega}
$$

In the event this condition does not hold, diffraction and refraction in the medium occur. The relations between electric and magnetic field intensities and flux densities in free space are [6]:

$$
\begin{aligned}
& \vec{B}=\mu_{0} \vec{H} \\
& \vec{D}=\epsilon_{0} \vec{E}
\end{aligned}
$$

Therefore, the resonant structures discussed here in a medium other than free-space have effective permittivity and permeability which contributes to the electric and magnetic field intensities 
and flux densities:

$$
\begin{aligned}
& \vec{B}=\mu_{0} \mu_{r} \vec{H} \\
& \vec{D}=\epsilon_{0} \epsilon_{r} \vec{E}
\end{aligned}
$$

The structure is assumed to be on a scale smaller than that of the excitation wavelength and may be heuristically defined with the homogeneity relation $p<\frac{\lambda}{4}$ where $p$ is the unit cell size.

\subsubsection{Array of cylinders}

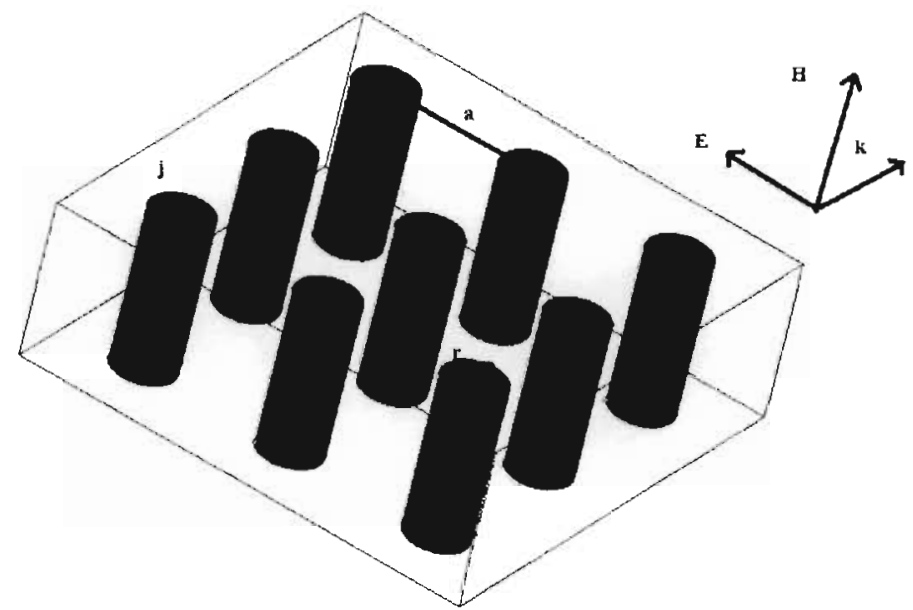

Figure 3.1 An array of metallic cylinders with an external magnetic field applied parallel to the cylinders.

If an external magnetic field $H_{0}$ is applied parallel to the cylinders and these cylinders have a perfect conducting surface, a current $\mathbf{j}$ per unit length flows. The field inside the cylinders is therefore [17]:

$$
H=H_{0}+\mathbf{j}-\frac{\pi r^{2}}{a^{2}} \mathbf{j}
$$

The second term on the right hand side of the above equation is due to the field caused by the rotating current, and the third term is the result of the depolarizing electric fields with sources at the top and bottom ends of the cylinders. One must calculate the total electromotive force around the 
circumference of the cylinder is [17]:

$$
\begin{aligned}
\mathrm{emf} & =-\pi r^{2} \mu_{0} \frac{\partial}{\partial t}\left[H_{0}+\mathbf{j}-\frac{\pi r^{2}}{a^{2}} j\right]-2 \pi r R \mathbf{j} \\
& =+i \omega \pi r^{2} \mu_{0}\left[H_{0}+\mathbf{j}-\frac{\pi r^{2}}{a^{2}} \mathbf{j}\right]-2 \pi r R
\end{aligned}
$$

where $R$ is the resistance of the cylinder surface per unit area. Both terms on the right hand side above are voltages. The latter term may be considered as loss due to resistance where voltage drop is current multiplied by resistance. The current $j$ per unit length may be solved for by assuming the net emf must balance to zero:

$$
\begin{aligned}
i \omega \pi r^{2} \mu_{0}\left[H_{0}+\mathbf{j}-\frac{\pi r^{2}}{a^{2}} \mathbf{j}\right]-2 \pi r R & =0 \\
j & =\frac{-H_{0}}{\left[1-\frac{\pi r^{2}}{a^{2}}\right]+i\left[\frac{2 r R}{\omega r \mu_{0}}\right]}
\end{aligned}
$$

To solve for effective permeability, equation (3.3) is used. Let $\bar{H}$ represent the average $\mathrm{H}$ field outside of the cylinders where there is no rotating current:

$$
\widetilde{H}=H_{0}-\frac{\pi r^{2}}{a} j
$$

Substituting in the derived equation for the current $\mathbf{j}$ per unit length (3.6) into (3.7) yields:

$$
\begin{aligned}
\bar{H} & =H_{0}-\frac{\pi r^{2}}{a^{2}} \frac{-H_{0}}{\left[1-\frac{\pi r^{2}}{a^{2}}\right]+i\left[\frac{2 r R}{\omega r \mu_{0}}\right]} \\
& =H_{0} \frac{1+i \frac{2 R}{\omega r \mu_{0}}}{\left[1-\frac{\pi r^{2}}{a^{2}}\right]+i \frac{2 R}{\omega r \mu_{0}}}
\end{aligned}
$$


The complex effective permeability of a cylinder array is [17]:

$$
\begin{aligned}
\mu_{e f f} & =\frac{\bar{B}}{\mu_{0} \bar{H}} \\
& =\frac{\mu_{0} H_{0}}{\mu_{0} \overline{\bar{H}}} \\
& =\frac{H_{0}}{H_{0} \frac{1+i \frac{2 R}{\omega r \mu_{0}}}{\left[1-\frac{\pi r^{2}}{a^{2}}\right]+i \frac{2 R}{\omega r \mu_{0}}}} \\
& =1-\frac{\pi r^{2}}{a^{2}}\left[i \frac{2 R}{\omega r \mu_{0}}\right]^{-1}
\end{aligned}
$$

\subsubsection{Capacitive array of sheets wound on cylinders}
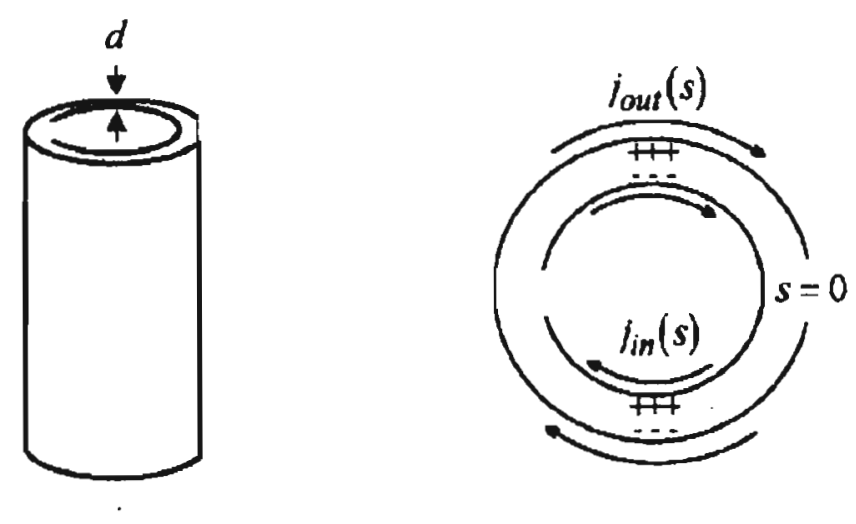

Figure 3.2 The cylinders are now seen to have an internal structure. The sheets are wound in a split ring fashion in such a way that current cannot flow freely. However, if the distance, $d$ is small enough, current may be induced. An increase in capacitance between sheets relates to an increase in current. Reference: [17]

The magnetic properties analyzed above change when introducing capacitive elements into the structure. An array of cylinders is used as before except that each cylinder is now built in a "split ring" fashion where two inverted rings may be seen when looking from the top down in figure 3.2.

The gap prevents current from flowing around any one ring. However, Pendry notes that the capacitance between the 2 rings enables current to flow [17]. A magnetic field parallel to the cylinder induces currents in the "split rings". The greater the capacitance between the sheets, the greater the current. 
The capacitive array of sheets has an effective permeability if the above process is repeated for the new model:

$$
\mu_{e f f}=1-\frac{\pi r^{2}}{a^{2}}\left[1+i \frac{2 R}{\omega r \mu_{0}}-\frac{3}{\pi^{2} \mu_{0} \omega^{2} C r^{3}}\right]^{-1}
$$

C represents the capacitance per unit area between the between the two sheets [17]:

$$
C=\frac{\epsilon_{0}}{d}=\frac{1}{d c_{0}^{2} \mu_{0}}
$$

Substituting the capacitance per unit area into the effective permeability model function one obtains:

$$
\mu_{e f f}=1-\frac{\frac{\pi r^{2}}{a^{2}}}{1+\frac{2 R i}{\omega r \mu_{0}}-\frac{3 d c_{0}^{2}}{\pi^{2} \omega^{2} r^{3}}}
$$

The numerator above represents the fractional volume of the cell occupied by the interior of the cylinder. There is a resonant frequency where the effective capacitance balances that of the inductance. A divergence or vertical asymptote is present in the effective permeability at this frequency [17]:

$$
\omega_{0}=\sqrt{\frac{3}{\pi^{2} \mu_{0} C r^{3}}}=\sqrt{\frac{3 d c_{0}^{2}}{\pi^{2} r^{3}}}
$$

At this resonant frequency, the effective permeability diverges to infinity as the limit of the denominator in (3.12) approaches zero. This can be seen by substituting (3.13) into (3.12).

Pendry also notes the existence of a magnetic plasma frequency, where thermal movement of electrons increase and become displaced. This displacement leads to a momentary creation of an electric field due to a small charge separation. However, the Coulomb force will lead to restoration of the electron's position (overshoot may occur). This process repeats resulting in oscillations.

$$
\omega_{m p}=\sqrt{\frac{3}{\pi^{2} \mu_{0} C r^{3}\left(1-\frac{\pi r^{2}}{a^{2}}\right)}}=\sqrt{\frac{3 d c_{0}^{2}}{\pi^{2} r^{3}\left(1-\frac{\pi r^{2}}{a^{2}}\right)}}
$$


Note the extra term from (3.13). This addition shows that the range over which resonance is observed up to the plasma frequency is dependent on the fraction of the unit cell structure that is not internal to any cylinder. In the frequency range the effective permeability is negative. In the region of negative $\mu_{e f f}$, if $\epsilon_{r}>0$, evanescent waves dominate and radiated EM waves cannot penetrate the metallic structure. At the plasma frequency, the real part of $\mu_{e f f}$ approaches zero and evanescent modes no longer dominate.

\subsubsection{Split ring resonator}
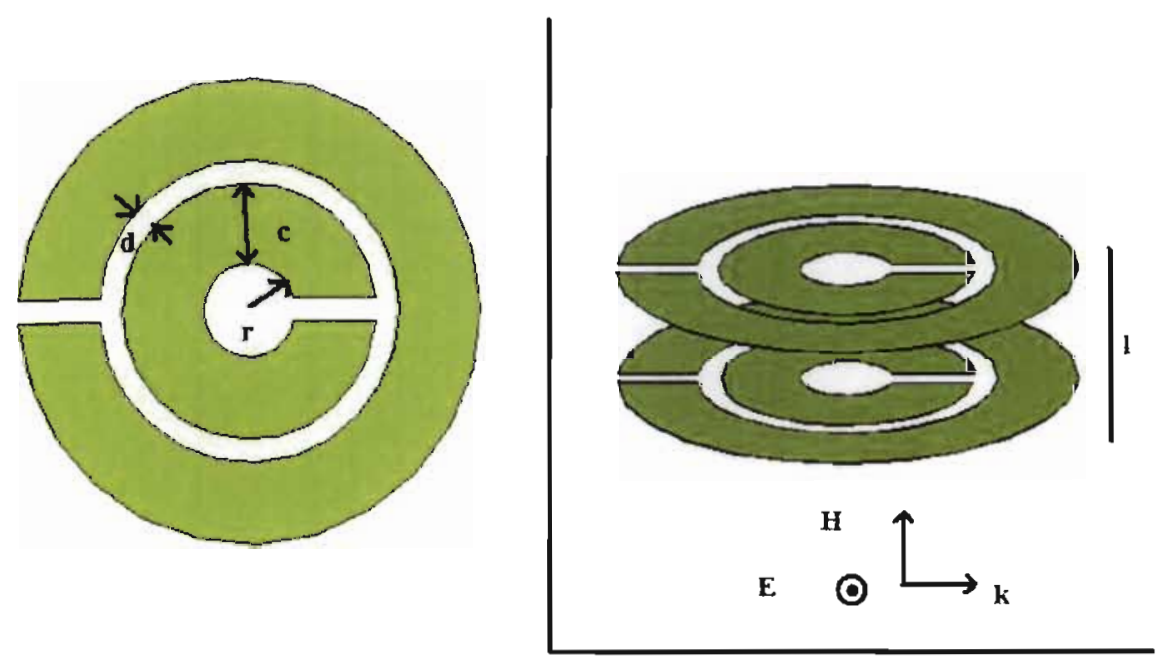

Figure 3.3 A thin flat disk shaped split ring resonator.

The above cylindrical designs have the disadvantage of having poor magnetic response when the magnetic field is not aligned parallel to the cylinders. Furthermore, if the electric field is also not parallel to the cylinders, the system responds similar an effective metal as current is free to flow across the length of the cylinders. However, this is fixed with the evolution of the structure into a disk shaped split ring resonator.

A split ring structure packaged in the form of flat disks can easily be made into arrays. A square array of flat disks may still be susceptible to magnetic field polarization but the continuous conduction path provided by the cylinders no longer exists. In order to calculate the effective permeability, the capacitance between the two elements of the split ring must be derived. Pendry makes the 
following assumptions:

- The distance to the inner ring $r>>$ the width of each ring $c$.

- $\ln \left(\frac{c}{d}\right)>>\pi$, where $d$ is the gap size between the two rings.

- Distance between any two rings must be less than $r$. Hence $l<r$.

The capacitance between a unit length of these parallel strips is defined in [17] as:

$$
C_{1}=\frac{\epsilon_{0}}{\pi} \ln \left(\frac{2 c}{d}\right)=\frac{1}{\pi \mu_{0} c_{0}^{2}} \ln \left(\frac{2 c}{d}\right)
$$

Therefore, the third assumption implies that the rings must be sufficiently close together. The magnetic force lines due to currents in the rings are the same as those in a continuous cylinder described earlier. Hence, the permeability relation somewhat resembles that of the capacitive array of sheets wound on cylinders.

Substituting (3.15) into (3.10):

$$
\mu_{c f f}=1-\frac{\frac{\pi r^{2}}{a^{2}}}{1+\frac{2 R i}{\omega r \mu_{0}}-\frac{3 d c_{0}^{2}}{\pi^{2} \omega^{2} r^{3}}}
$$

A divergence in the effective permeability (3.16) occurs at:

$$
\omega_{0}=\sqrt{\frac{3 l c_{0}^{2}}{\pi \ln \left(\frac{2 c}{d} r^{3}\right)}}
$$

The variable $l$ above represents the distance between two rings. One can assume this value to be less than the inner radius of the split ring. This variable may be substituted for by the gap size between rings $d$ which is also less than the inner radius of the SRR.

The permeability relation for the circular SRR is implemented in Matlab where the dimension variables may be altered to examine the effect of said variables on the permeability resonance frequency. The Matlab script calculating and plotting the theoretical resonance frequency in the effective permeability may be found in Appendix A. 

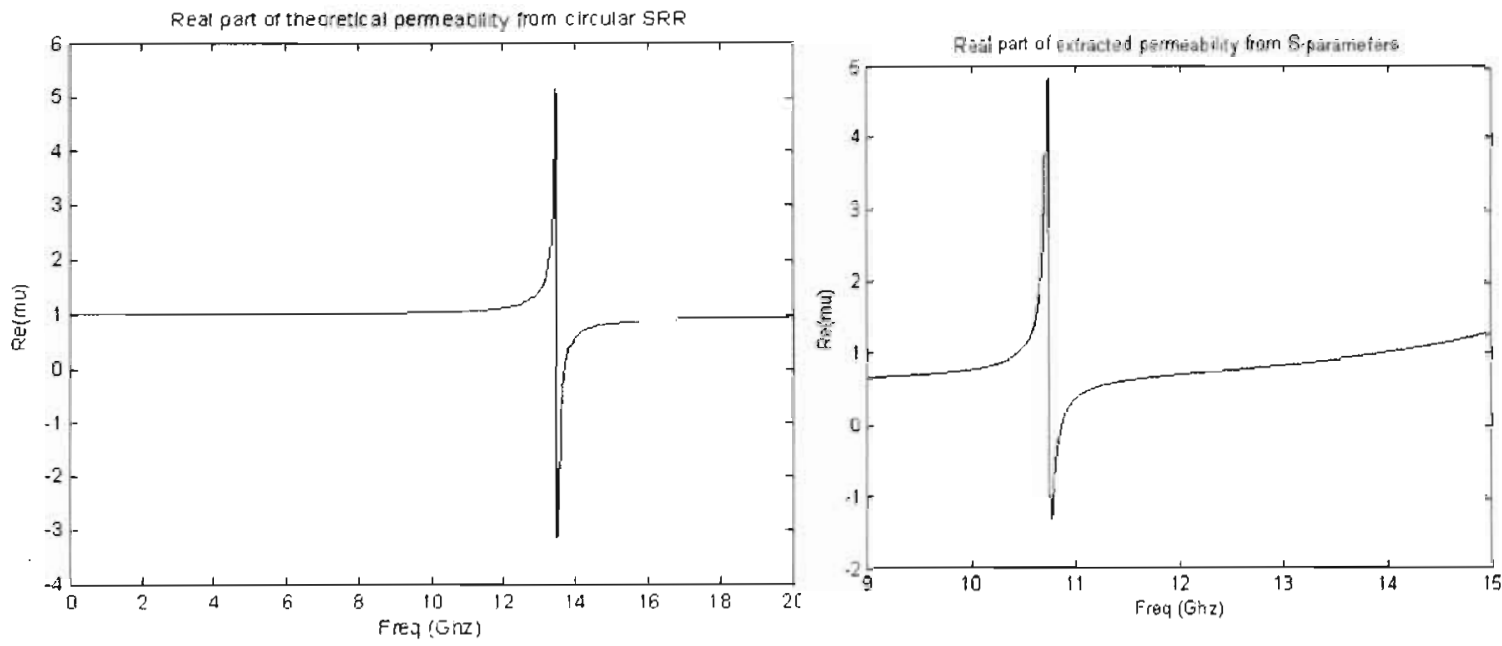

Figure 3.4 A circular split ring resonator with the variables: $a=10 \mathrm{~mm}, c=1 \mathrm{~mm}, d=0.1 \mathrm{~mm}$, $\mathrm{l}=2 \mathrm{~mm}$ and $\mathrm{r}=2 \mathrm{~mm}$ is evaluated in Matlab using the effective permeability relation (3.16) on the left. The equation predicts a resonance in permeability at approximately $13.5 \mathrm{GHz}$. HFSS simulations to obtain S-paramaters and subsequent paramater extraction predicts a resonance in the real part of the permeability at $11 \mathrm{GHz}$ (right plot). HFSS simulations of a circular SRR are presented in more detail in section 3.2.

\subsection{Circular SRR inclusion simulation in Ansoft HFSS}

\subsubsection{Unit cell synthesis in HFSS}

Ansoft HFSS version 10 is used to synthesize and simulate metamaterial structures in this project. Previous work in [10] introduced the possibility of reducing simulation time by simulating a single or small number (much less than the possible hundreds of inclusions embedded in a full structure) of inclusions embedded in a dielectric medium via the PEC/PMC waveguide method. In this method, two waveports are placed on the entrance and exit faces of the dielectric slab. The "integration line" feature in HFSS defines the fundamental E field mode of the wave illuminating the entrance. A PEC symmetry plane acts as a metallic layer. Hence the E field tangential to the surface is zero. The E field must be normal to the surface or plane. The PMC (defined as Perfect H in HFSS) denote surfaces or planes where the tangential magnetic field is zero; thus the H-field must be normal to this surace or plane. Hence, by placing PEC and PMC symmetry planes as such shown in figure 3.5, the $\mathrm{E}$ and $\mathrm{H}$ vectors are orthogonal to each another. The wave emanating from waveport 1 is thus a plane wave. As a result, it is not necessary to simulate a full metamaterial structure in 
transmission testing. The simulation space may be reduced down to one or a small number of unit cells. Depending on the size of the inclusions, more than five or six inclusions may lead to longer simulation and adaptive mesh refinement times. In figure 3.5, Arrows indicate direction of EM wave propagation. A side view shows the vector direction of the plane wave on the air / metamaterial slab boundary. Perfect E (PEC) (XY plane) and Perfect H (PMC) (YZ plane) boundary faces are shown should the whole slab be simulated in HFSS. Waveports are placed on the entrance and exit faces in the XZ plane.

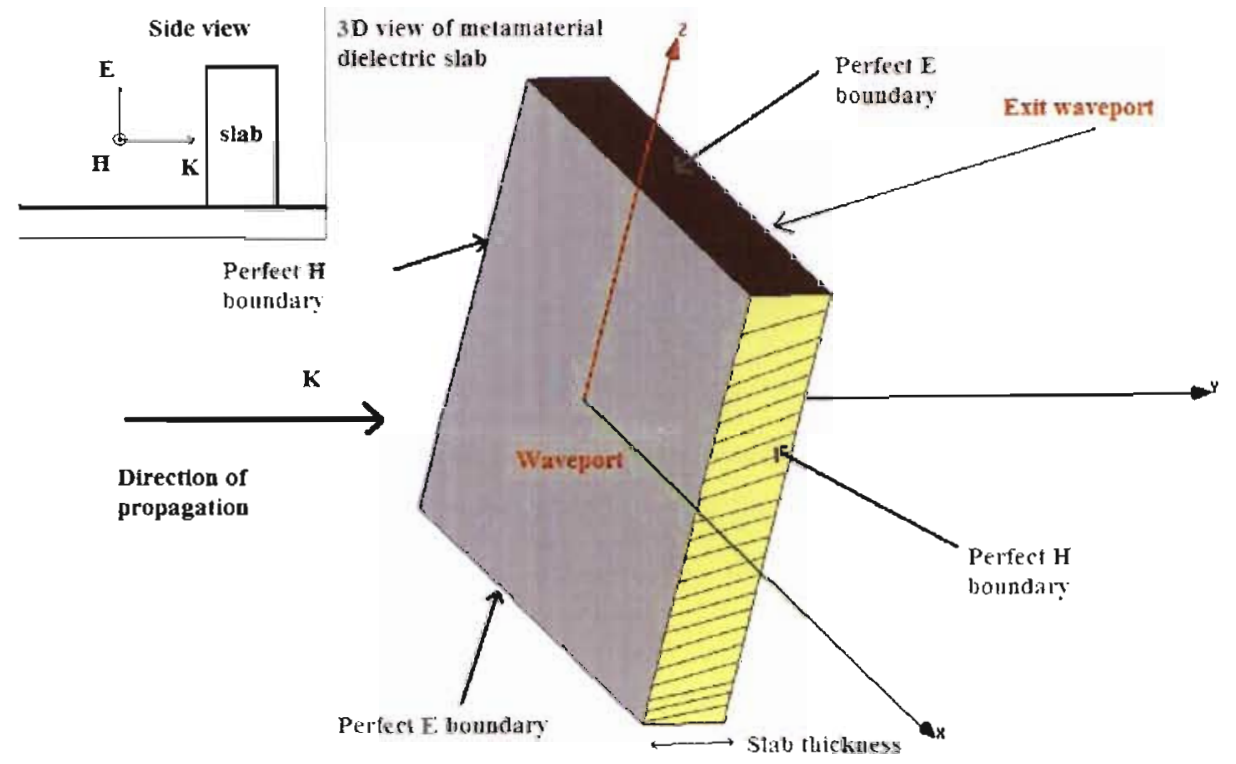

Figure 3.5 Outline of a complete metamaterial slab. 


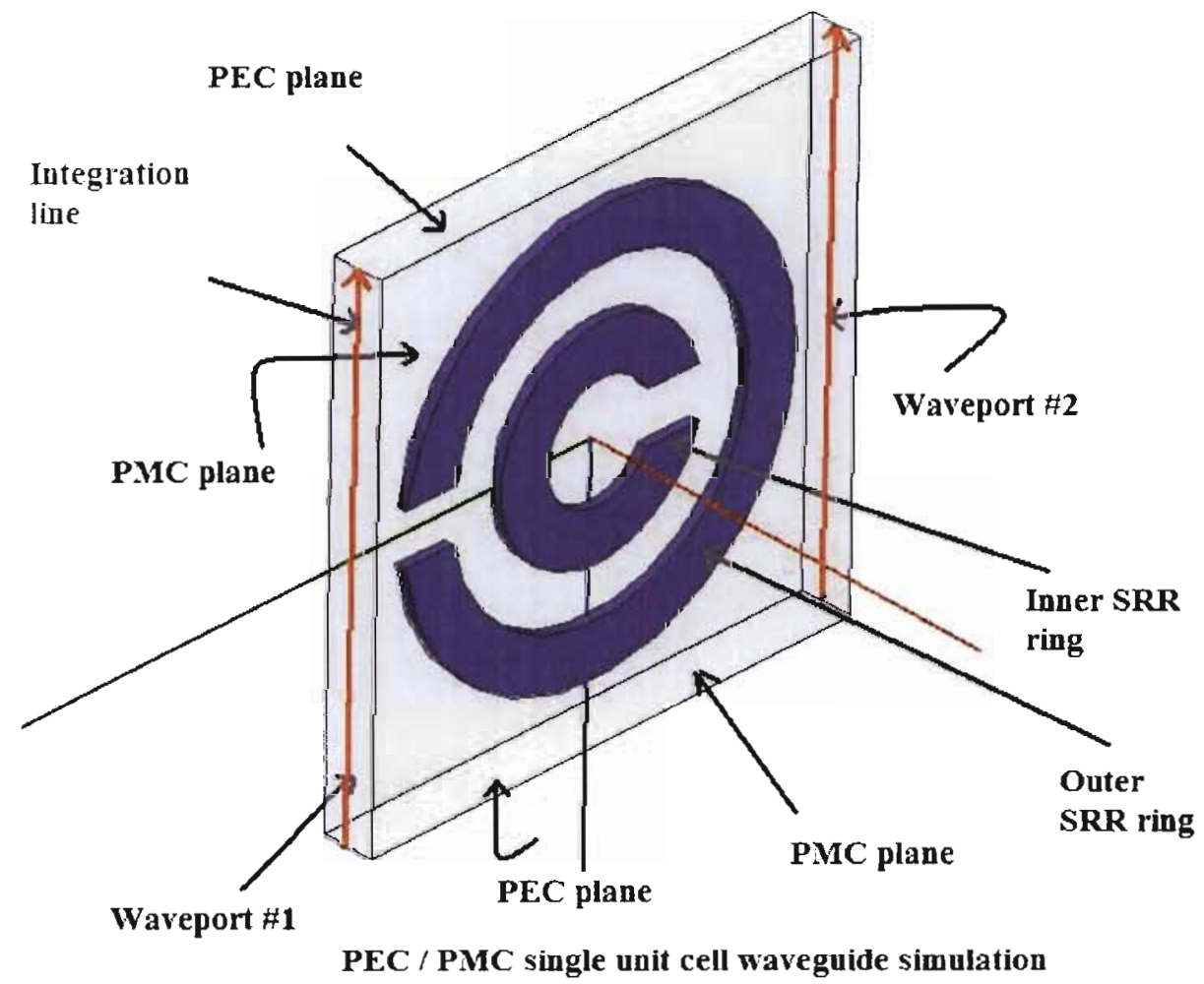

Figure 3.6 Single metamaterial cell PEC/PMC wavegulde configuration in HFSS. The waveport integration lines are shown. PEC/PMC faces are also indicated. 


\subsubsection{Simulation results}

To verify the accuracy and utility of Pendry's analytical equations for a circular split ring resonator, the unit cell in figure 3.6 was simulated. Table 3.1 depicts theoretical and simulated resonant frequencies for multiple unit cell dimensions. The use of variables in HFSS simplifies the adjustment of dimensions such as dielectric thickness, ring radii and gap sizes. For the majority of simulations, Pendry's criteria for unit cell dimensions are maintained. Results show the Pendry equations provide only a very rough approximation and that HFSS simulations are absolutely necessary to characterize a SRR inclusion. The last bolded row exhibits the case where pendry's unit cell dimension criteria are not met. Here the distance between stacked rings is much greater than the inner radius. In this case, theoretical values are disparate from the simulated results. Note that the second resonance in the S-parameters is used for $f_{0}$ as they correspond more closely to the theoretical equations proposed by Pendry. Simulations are conducted in both a vacuum $\left(\epsilon_{r}=1\right)$ and Rogers Duroid 5880 $\left(\epsilon_{r}=2.2\right)$ dielectric media. In Figure 3.7, two resonances are shown to exist. However, Pendry's analysis only predicts the second higher frequency resonance in $S_{11}$ and $S_{21}$. At these permeability resonances, the structure acts like an open circuit where $S_{11}$ increases in magnitude and $S_{21}$ drops off significantly.

- The distance to the inner ring $r>>$ the width of each ring $c$.

- $\ln \left(\frac{c}{d}\right)>>\pi$, where $d$ is the gap size between the two rings.

- Distance between any 2 rings must be less than $r$. Hence dielectric thickness must be less than $r$ (inner radius).

\subsubsection{Parameter variations}

To investigate the effect of the split ring resonator size (in terms of overall radius and diameter), HFSS is used to calculate the S-Parameters for a split ring resonator unit cell. In this parametric analysis, the copper trace width, gap size and dielectric height are kept constant. Furthermore, the boundary unit cell dimensions are fixed. Only the outer radius is varied. In doing so, the overall 


\begin{tabular}{||c|c|c|c|c|c|c|c|c|c|c||}
\hline $\begin{array}{c}\text { Outer } \\
\text { radius } \\
\mathrm{mm}\end{array}$ & $\begin{array}{c}\text { trace } \\
\text { width } \\
\mathrm{mm}\end{array}$ & $\begin{array}{c}\text { gap } \\
\text { size } \\
\mathrm{mm}\end{array}$ & $\begin{array}{c}\text { Inner } \\
\text { radius } \\
\mathrm{mm}\end{array}$ & $\begin{array}{c}\text { Diel } \\
\text { thick. } \\
\mathrm{mm}\end{array}$ & $\begin{array}{c}\text { Th } f_{0} \\
\text { vac } \\
\mathrm{Ghz}\end{array}$ & $\begin{array}{c}\text { Th } f_{0} \\
\text { dur } \\
\mathrm{GHz}\end{array}$ & $\begin{array}{c}\text { Simul } f_{0} \\
\text { vac } \\
\mathrm{GHz}\end{array}$ & $\begin{array}{c}\text { Simul } f_{0} \\
\text { dur } \\
\mathrm{GHz}\end{array}$ & $\begin{array}{c}\text { \%diff } \\
\text { vac }\end{array}$ & $\begin{array}{c}\text { \%diff } \\
\text { dur }\end{array}$ \\
\hline 4.5 & 1 & 0.1 & 2.4 & 1 & 7.25 & 4.9 & 10 & 6.5 & 37.9 & 32.7 \\
\hline 4.1 & 1 & 0.1 & 2 & 2 & 13.4 & 9.08 & 11.5 & 7.5 & 14.2 & 17.4 \\
\hline 3 & 0.7 & 0.1 & 1.5 & 1 & 15.6 & 10.54 & 13.3 & 10.3 & 14.7 & 2.3 \\
\hline 2.5 & 0.5 & 0.1 & 1.4 & 1 & 18.5 & 12.5 & 17.4 & 11.9 & 5.95 & 4.8 \\
\hline $\mathbf{4 . 1}$ & $\mathbf{1}$ & $\mathbf{1}$ & $\mathbf{1 . 1}$ & $\mathbf{3}$ & $\mathbf{8 4 . 1}$ & $\mathbf{5 6 . 7}$ & $\mathbf{2 5 . 5}$ & $\mathbf{1 6 . 7}$ & $\mathbf{6 9 . 7}$ & $\mathbf{7 0 . 5}$ \\
\hline
\end{tabular}

Table 3.1 HFSS simulation results for multiple circular SRR unit cell dimensions.

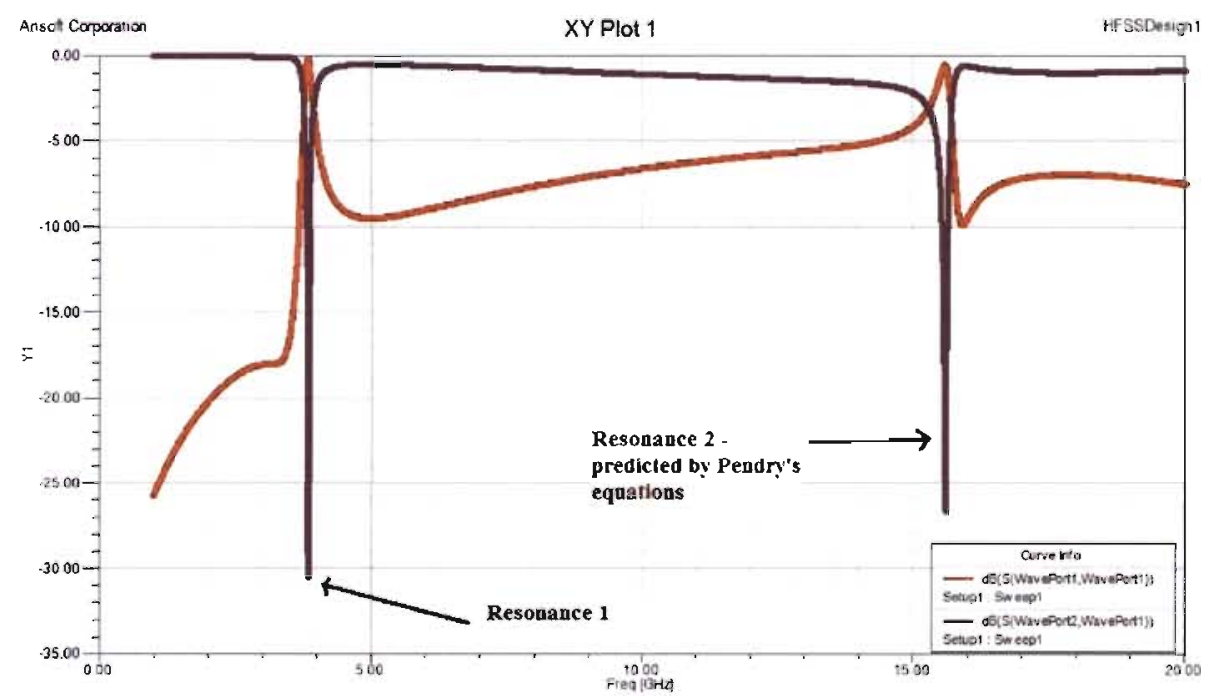

Figure 3.7 S-paramaters obtained from HFSS of a circular SRR with parameters: $r=$ $1.4 \mathrm{~mm}$, trace width $=0.5 \mathrm{~mm}$, gap width $=0.1 \mathrm{~mm}$, lattice spacing (dielectric spacing) $=$ $1 \mathrm{~mm}$ and substrate dielectric permittivity $=2.2$.

diameter of the SRR changes, hence its overall size. Larger trace lengths exhibit larger inductance and capacitive coupling between the two rings. Based on the standard resonant frequency equation: $f_{0}=\frac{1}{2 \pi \sqrt{L \cdot C}}$, increasing both the capacitance and inductance reduces the resonant frequency. Simulation results are shown in Figure 3.8. In the simulation investigating radius lengths, trace width is kept constant at $0.5 \mathrm{~mm}$. Gap sizing is $0.1 \mathrm{~mm}$. Unit cell boundaries unchanged at $5 \mathrm{~mm} \times 5 \mathrm{~mm}$ with a dielectric height of $1 \mathrm{~mm}$. The Dielectric is Rogers Duroid $8500 \epsilon_{r}=2.2$ 


\section{Resonant frequency of SRR as a function of SRR outer diameter}

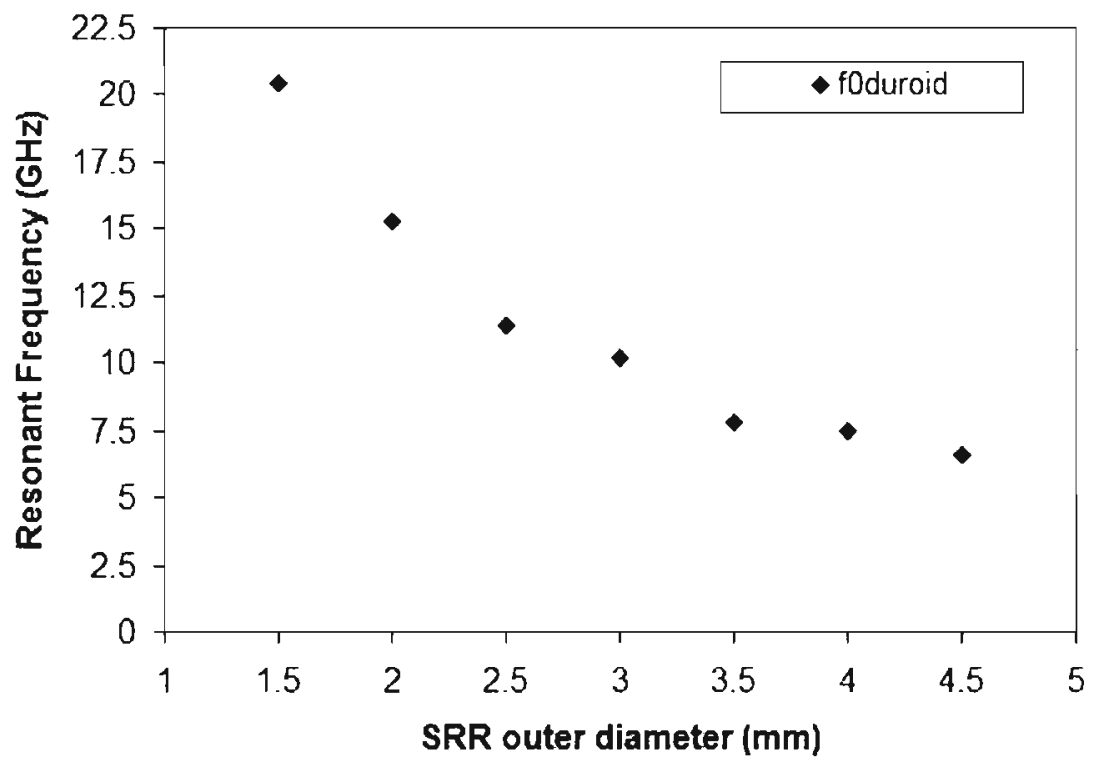

Figure 3.8 Resonant Frequency of circular SRR for multiple outer radius lengths.

Another parameter of interest is the substrate permittivity $\epsilon_{r}$. Results depicted in Figure 3.9 show that resonant frequency of an SRR decreases as $\epsilon_{r}$ increases. This is important as the choice of substrate permittivity needs to be finalized before performing final design simulations. Furthermore to compensate for higher permittivities, other parameters such as SRR outer radius, trace widths or gap widths need alteration.

Additional variable parameters include copper trace widths and gap widths. Copper trace width is represented by the variable ' $c$ ' in equation (3.17); the argument of a natural log function. Increasing ' $c$ ', decreases the resonant frequency. The pattern is exponential in nature due to the natural log function. The same analysis may be applied to the gap widths. Increasing the gap width reduces the inner ring radius $(r)$ and hence also increases the value of the natural $\log$ function. As a result, the resonant frequency increases exponentially. 


\section{Resonance frequency of Circular SRR as a function of Dielectric permittivity}

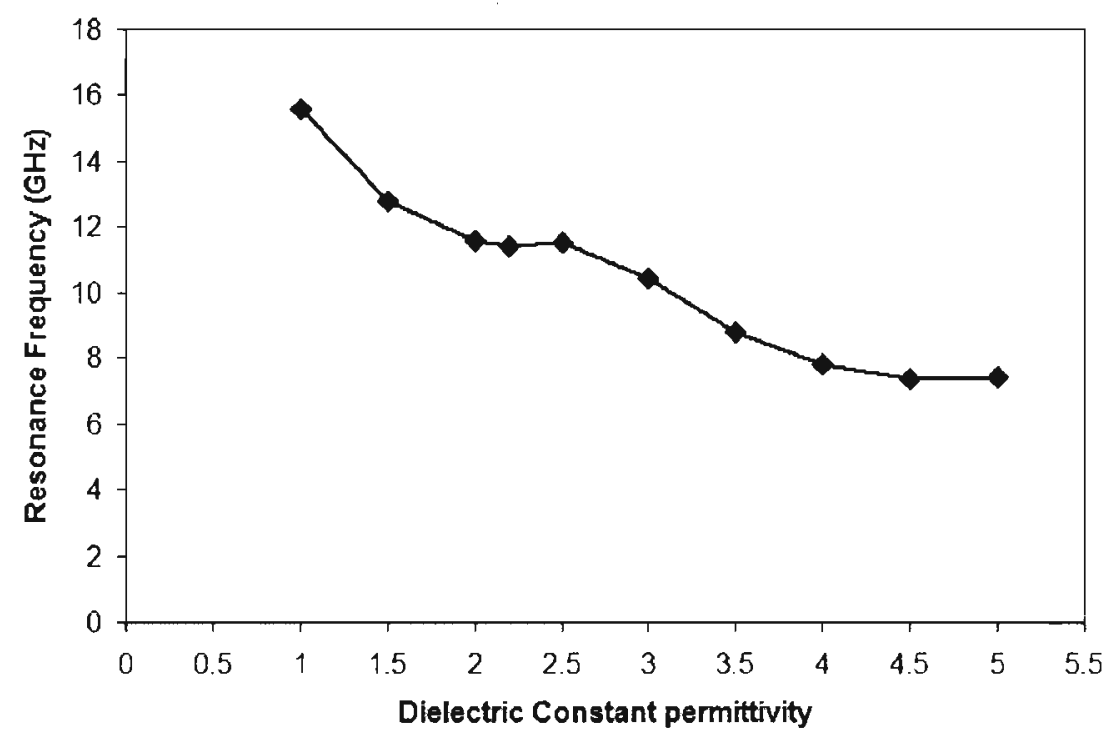

Figure 3.9 Resonant Frequency of circular SRR for varying substrate dielectric constants. SRR outer radius kept constant at $2.5 \mathrm{~mm}$. Trace width is $0.5 \mathrm{~mm}$. Gap sizing is $0.1 \mathrm{~mm}$. Unit cell boundaries unchanged at $5 \mathrm{~mm} \times 5 \mathrm{~mm}$ with a dielectric height of $1 \mathrm{~mm}$.

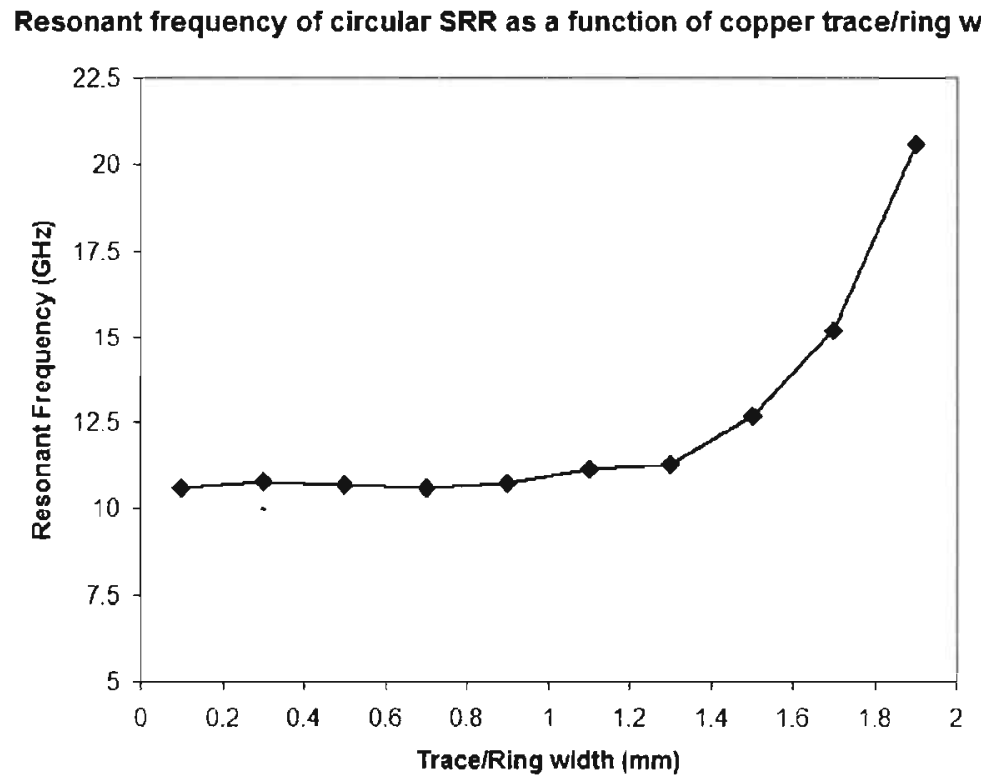

Figure 3.10 Resonant Frequency of circular SRR for varying copper trace widths. SRR outer radius kept constant at $4.0 \mathrm{~mm}$. Gap sizing is $0.1 \mathrm{~mm}$. Unit cell boundaries unchanged at $5 \mathrm{~mm} \times 5 \mathrm{~mm}$ with a dielectric height of $1 \mathrm{~mm}$. The Dielectric is Rogers Duroid $8500 \epsilon_{r}=2.2$ 
Resonant frequency of circular SRR as a function of gap width

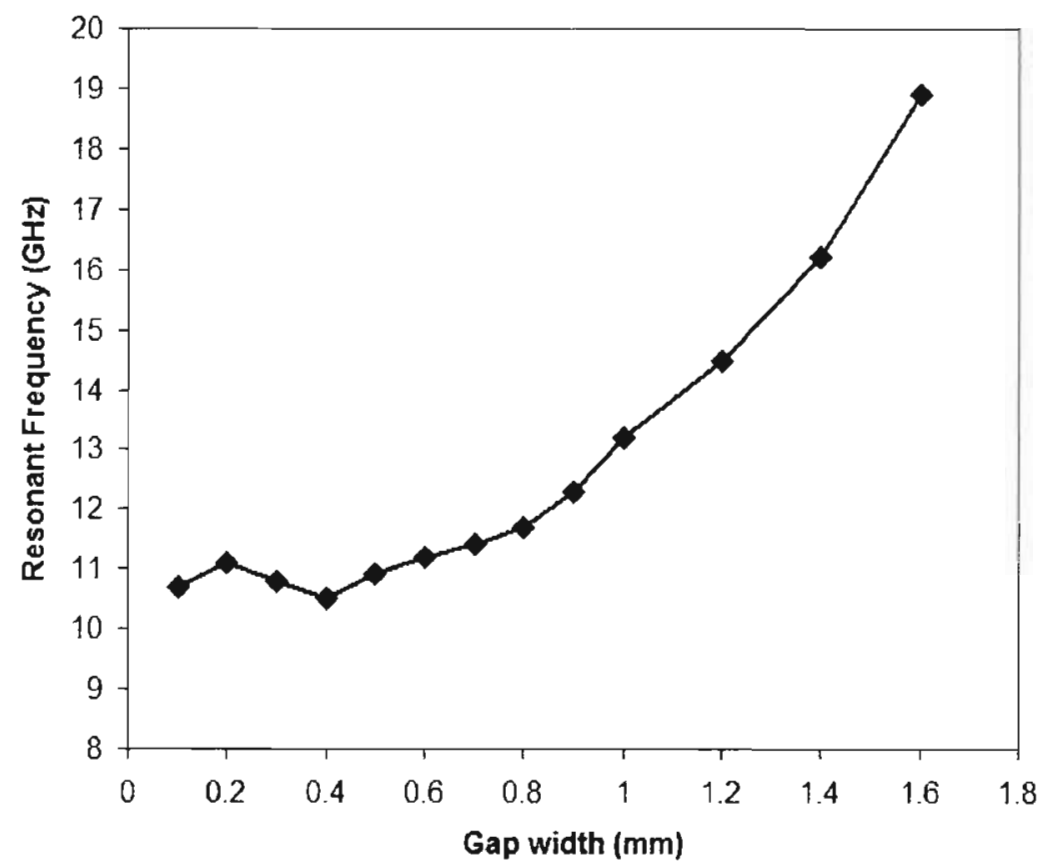

Figure 3.11 Resonant Frequency of circular SRR for varying gap widths. SRR outer radius kept constant at $4.0 \mathrm{~mm}$. Trace width is $0.5 \mathrm{~mm}$. Unit cell boundaries unchanged at $5 \mathrm{~mm} \times 5 \mathrm{~mm}$ with a dielectric height of $1 \mathrm{~mm}$. The Dielectric is Rogers Duroid 8500 $\epsilon_{r}=2.2$

\subsection{Square / Quadrilateral SRR inclusion simulation in Ansoft HFSS}

\subsubsection{Unit cell synthesis in HFSS}

Unit synthesis is almost identical to that of the circular SRR. However, instead of using cylinders with formed gaps using boolean operations in HFSS, the box drawing tool is especially useful for creating the structure. Boolean operations may be used to form gaps between and inside rings. A PEC/PMC waveguide setup for a single unit cell is again used. The Perfect E and Perfect $\mathrm{H}$ symmetry planes are unchanged.

\subsubsection{Parameter variations}

For the parameter variations of the Quadrilateral SRR unit cell shown in the graphs below, the first two major resonances are shown. Both resonances show a peak in the reflection of the unit cell and 


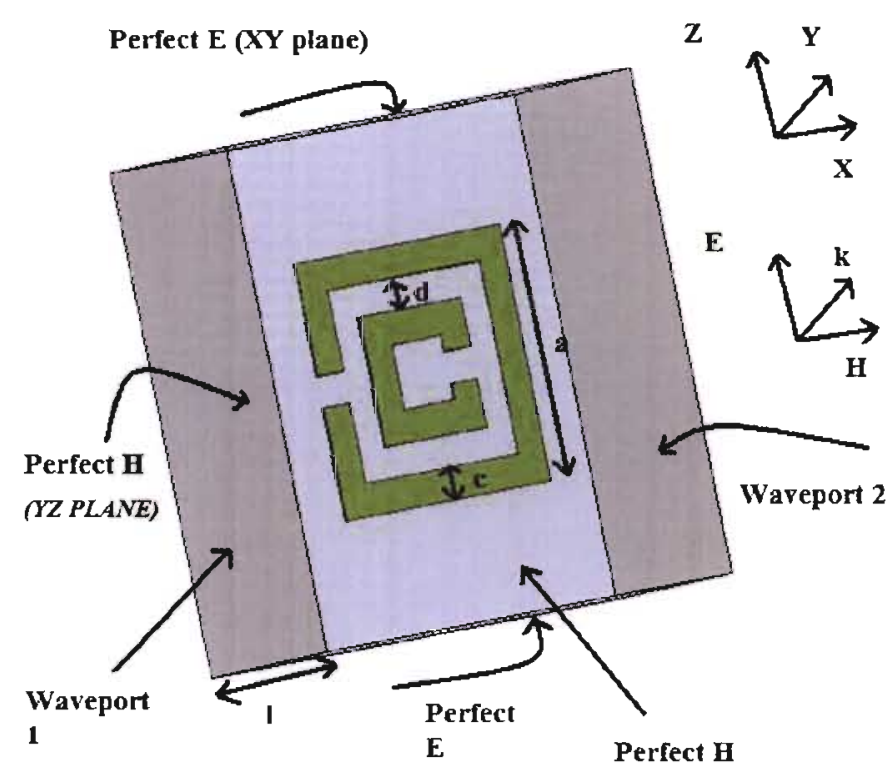

Figure 3.12 Single unit cell of a square SRR in the PEC/PMC waveguide configuration for simulation in HFSS.

a minimum in transmission. Hence the unit cell is acting like an open circuit at these two resonance frequencies. This is characteristic of a unit cell with negative permeability only as reported by Ziolkowski in [10]. Figure 3.13 shows the change in resonance frequency as outer radius length is varied. Trace widths are kept constant at $0.5 \mathrm{~mm}$. Gap sizing is $0.1 \mathrm{~mm}$. Unit cell boundaries are unchanged at $5 \mathrm{~mm} \times 5 \mathrm{~mm}$ with a dielectric height of $1 \mathrm{~mm}$. The Dielectric is Rogers Duroid 8500 $\epsilon_{r}=2.2$. Furthermore, Figure 3.14 shows a similar response in the resonance frequency to changes in the inclusions's copper trace width. SRR outer radius is kept constant at $4.0 \mathrm{~mm}$. Gap sizing is $0.1 \mathrm{~mm}$. Unit cell boundaries unchanged at $5 \mathrm{~mm} \times 5 \mathrm{~mm}$ with a dielectric height of $1 \mathrm{~mm}$. The Dielectric is Rogers Duroid $8500 \epsilon_{r}=2.2$

Figure 3.15 depicts the effect of split ring gap widths on the measured S-parameter resonance frequency. The gaps of the outer and inner rings are identical to simplify analysis and remove the addition of extra variables. SRR outer radius is kept constant at $4.0 \mathrm{~mm}$. Trace width is $0.5 \mathrm{~mm}$. Unit cell boundaries are unchanged at $5 \mathrm{~mm} \times 5 \mathrm{~mm}$ with a dielectric height of $1 \mathrm{~mm}$. The Dielectric is Rogers Duroid $8500 \epsilon_{r}=2.2$. 
Resonance Frequency as a function of split ring outer diameter for a square/quadrilateral SRR

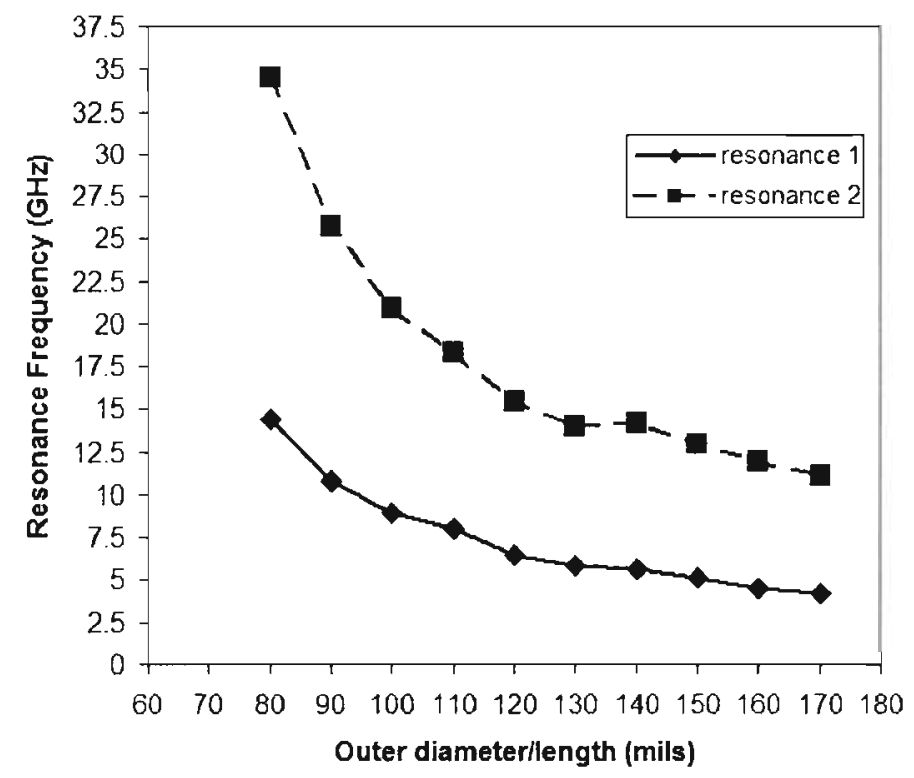

Figure 3.13 Resonant Frequency of a square SRR with multiple outer radius lengths.

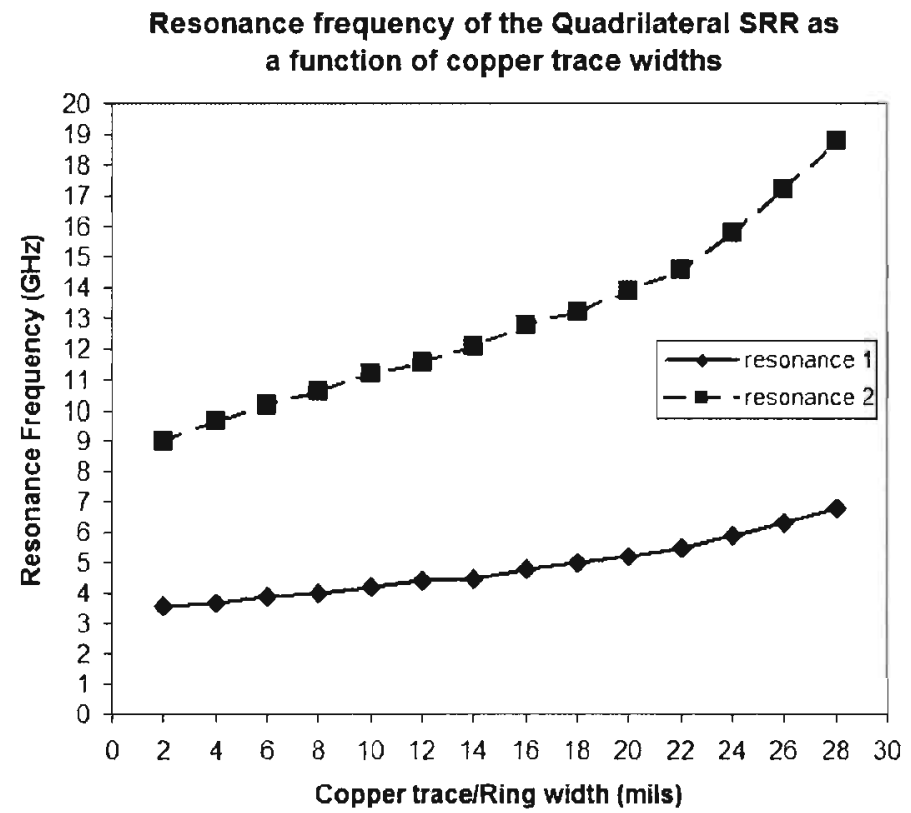

Figure 3.14 Resonant Frequency of square SRR for varying copper trace widths.

Results follow the same correlations and relations between the resonance frequencies and square SRR parameters as seen in the earlier HFSS simulation results of circular SRRs. For the final meta- 


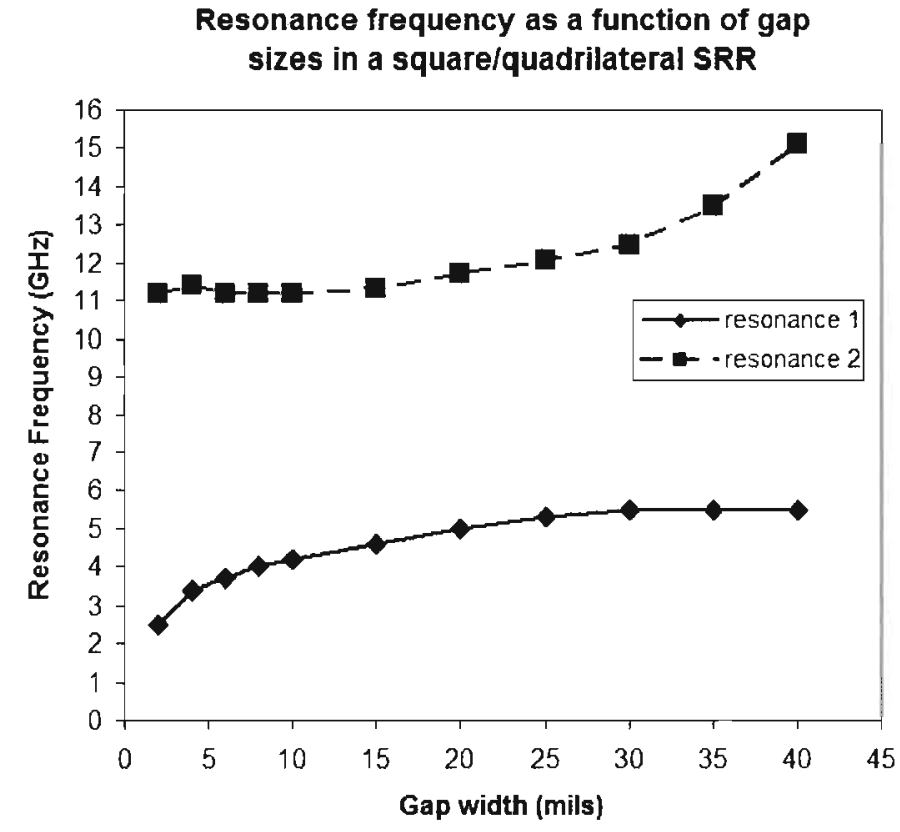

Figure 3.15 Resonant Frequency of square SRR for varying gap widths.

material designs discussed in a Chapter 9 , parameters may be adjusted to meet resonance frequency requirements. The parametric analysis results here provide an intuitive way to adjust the parameters to meet resonance goals. 


\section{Chapter 4}

\section{Wire and strip structures to realize}

\section{negative permittivity}

While the split ring resonator reacts to the presence of a magnetic field thereby affecting the permeability of the bulk material, it is also necessary to present inclusions that produce dielectric responses based on the illuminating E-field. Such structures may be designed to exhibit negative permittivity $\left(\epsilon_{r}\right)$ over a specified frequency range.

Metals exhibit a plasma resonance of their electrons when when illuminated by electromagnetic radiation of a certain frequency. In the ideal case, the relative permittivity of a metal may be described by the relation [18]:

$$
\begin{aligned}
\epsilon_{\text {metal }} & =1-\frac{\omega_{p}^{2}}{\omega^{2}} \\
\omega_{p}^{2} & =\frac{n e^{2}}{\epsilon_{0} m_{e}}
\end{aligned}
$$

The plasma frequency $\omega_{p}$, is given in terms of the electron density, $\mathrm{n}$, the electron mass, $m_{e}$, and charge, e. For frequencies less than the plasma frequency, metal exhibits negative permittivity. In regions of exclusive negative permittivity evanescent modes dominate. In this region radiation does not penetrate the metal. Furthermore, the ideal equation above is not valid in the presence of losses, $\gamma$. Equations (4.1) and (4.2) are state that metals exhibit negative permittivitty below the plasma resonance frequency. Hence, a negative permittivity inclusion designed to exhibit a dielectric 
response to an, incident $\mathbf{E}$ field must be illuminated by radiation below the plasma frequency.

$$
\begin{aligned}
\epsilon_{\text {metal }} & =1-\frac{\omega_{p}^{2}}{\omega(\omega+i \gamma)} \\
\mathrm{i} & =\sqrt{-1}
\end{aligned}
$$

\subsection{Thin wire structures}

If a periodic structure of thin wires is to be considered, it is important to consider the case of an illuminating E-field parallel to the wire lines. In the previous chapter, cylinders were considered as inclusions to achieve a magnetic response from the presence of a H-field. In this case, thin wires are considered. According [18], in thin wires, the average electron density is reduced because only part of the unit cell or collection of wires in a dielectric medium is occupied by a metal. Thus the overall average density of electrons, $n_{e f f}$, in wires of radius $r$ with a density $n$ in the wires themselves and wire seperation a may be represented as:

$$
n_{e f f}=n \frac{\pi r^{2}}{a^{2}}
$$

Furthermore, the effective electron mass is enhanced due to magnetic effects. The flow of current, $I$ produces a magnetic field around the wire:

$$
\begin{aligned}
& H(R)=\frac{I}{2 \pi R}=\frac{\pi r^{2} n v e}{2 \pi R} \\
& \mathbf{H}(R)=\mu_{0}^{-1} \nabla \times \mathbf{A}
\end{aligned}
$$

where

$$
A(R)=\frac{m u_{0} \pi r^{2} n v e}{2 \pi} \ln \left(\frac{a}{R}\right)
$$

In (4.4), $R$ represents field distance from the wire, $v$ is the mean electron velocity and $a$ is the lattice constant (separation between wires) (see Figure 12.5). The momentum contribution to 
electrons in a magnetic field is $\mathbf{e A}$. Per unit length the momentum of the wire is:

$$
\begin{aligned}
e A(r) \pi r^{2} n A(r) & =\frac{\mu_{0} e^{2}\left(\pi r^{2} n\right)^{2} v}{2 \pi} \ln \left(\frac{a}{r}\right) \\
& =m_{e f f} \pi r^{2} n v
\end{aligned}
$$

The effective mass of the electrons is $m_{e f f}$. One can re-arrange (4.5) to obtain $m_{e f f}$ :

$$
m_{e f f}=\frac{\mu_{0} e^{2} \pi r^{2} n}{2 \pi} \ln \left(\frac{a}{r}\right)
$$

The plasma frequency of the thin wire structure defines the upper limit for the domain in which the bulk permittivity is negative. From (4.1):

$$
\begin{aligned}
\epsilon_{0} & =\frac{1}{\mu_{0} c^{2}} \\
w_{p}^{2} & =\frac{n_{e f f} e^{2}}{\epsilon_{0} m_{e f f}}=\frac{n \frac{\pi r^{2}}{a^{2}} e^{2}}{\epsilon_{0} \frac{\mu_{0} e^{2} \pi r^{2} n}{2 \pi} \ln \left(\frac{a}{r}\right)} \\
& =\frac{2 \pi}{a^{2} \ln \left(\frac{a}{r}\right) \epsilon_{0} \mu_{0}} \\
& =\frac{2 \pi}{a^{2} \ln \left(\frac{a}{r}\right) \frac{1}{\mu_{0} c_{0}^{2}} \mu_{0}} \\
& =\frac{2 \pi c_{0}^{2}}{a^{2} \ln \left(\frac{a}{r}\right)}
\end{aligned}
$$

The above relation is used to determine the plasma frequency of the rod structures. The following section tabulates results obtained from HFSS when simulating an array of thin wire rods.

\section{Simulation of rod structures in HFSS}

A unit cell is constructed in the PEC/PMC waveguide format as previously discussed. Several cylinders composed of copper are used to create the wire rod array. The cylinders are seperated by the distance $a$, with radius, $r$. The rods must contact the top and bottom faces of the unit cell. As the top and bottom faces of the cell are Perfect E planes approximating metal sheets, the structure may be considered a parallel plate waveguide [19]. In practice, to ensure there is a 
sufficient dielectric response to the electric field, the rods must connect to the metal sheets of the parallel plate waveguide. In the case of simulations they must touch the top and bottom face of the PEC/PMC waveguide but must not completely intersect it otherwise HFSS will produce errors.

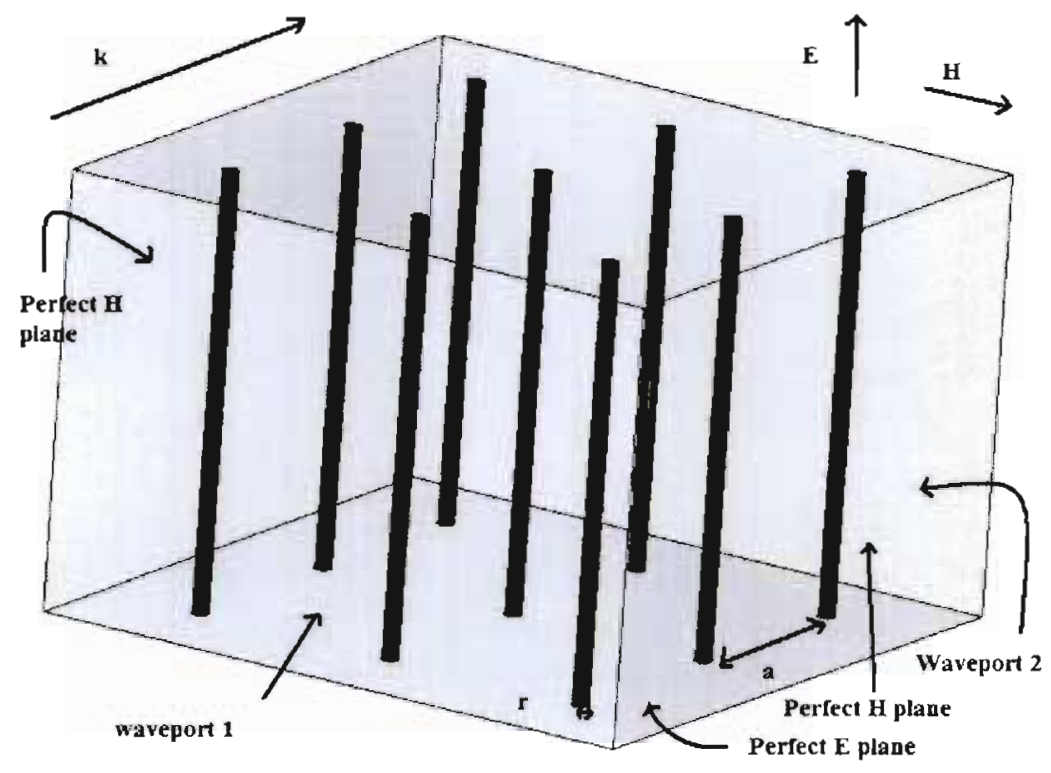

Figure 4.1 HFSS structure example for an array of thin wire rods.

The plasma frequency is visible in in transmission data when a peak in transmission $\left(S_{21}\right)$ is seen. An example of transmission data calculated by full-wave simulation in HFSS is given in figure 4.2.

Several simulations with different cylinder radii and lattice spacing are considered in table 4.1. The theoretical results in an air dielectric are compared to the HFSS solutions. Note the large percentage difference between simulated and theoretical results. The radius of the cylinders used are not "thin" by J B Pendry's standards. Thicker wires undergo higher resistive losses at microwave frequencies which tends to increase the plasma frequency.

Table 4.1 shows an unusually high percentage difference between theoretical and simulated data. This is due to the diameter of the cylindrical wires. Even at $0.1 \mathrm{~mm}$, they cannot be considered thin enough to be treated as ideal in equation (4.1). The table shows percentage difference in theoretical to simulated results decreases with radius. However, simulation of radii less than $0.1 \mathrm{~mm}$ becomes difficult in HFSS as the solver finds it impossible to fit tetrahedra to extremely small structures. 


\begin{tabular}{|c|c|c|c|c||}
\hline $\mathrm{r}(\mathrm{mm})$ & $\mathrm{a}(\mathrm{mm})$ & theorerical $f_{p}(\mathrm{GHz})$ & simul $f_{p}(\mathrm{GHz})$ & \%difference \\
\hline 0.1 & 5 & 12.1 & 17.7 & 31.63 \\
\hline 0.2 & 5 & 13.3 & 20 & 33.3 \\
\hline 0.5 & 5 & 15.8 & 24.3 & 35.08 \\
\hline 1.5 & 5 & 21.8 & 47.2 & 53.8 \\
\hline 0.1 & 2.5 & 26.7 & 39.6 & 32.6 \\
\hline 0.1 & 10 & 5.58 & 8.7 & 35.8 \\
\hline
\end{tabular}

Table 4.1 Theoretical and simulated plasma frequencies for thin $3 \times 3$ wire rod structure unit cells of various wire radii and lattice spacings in an air medium.

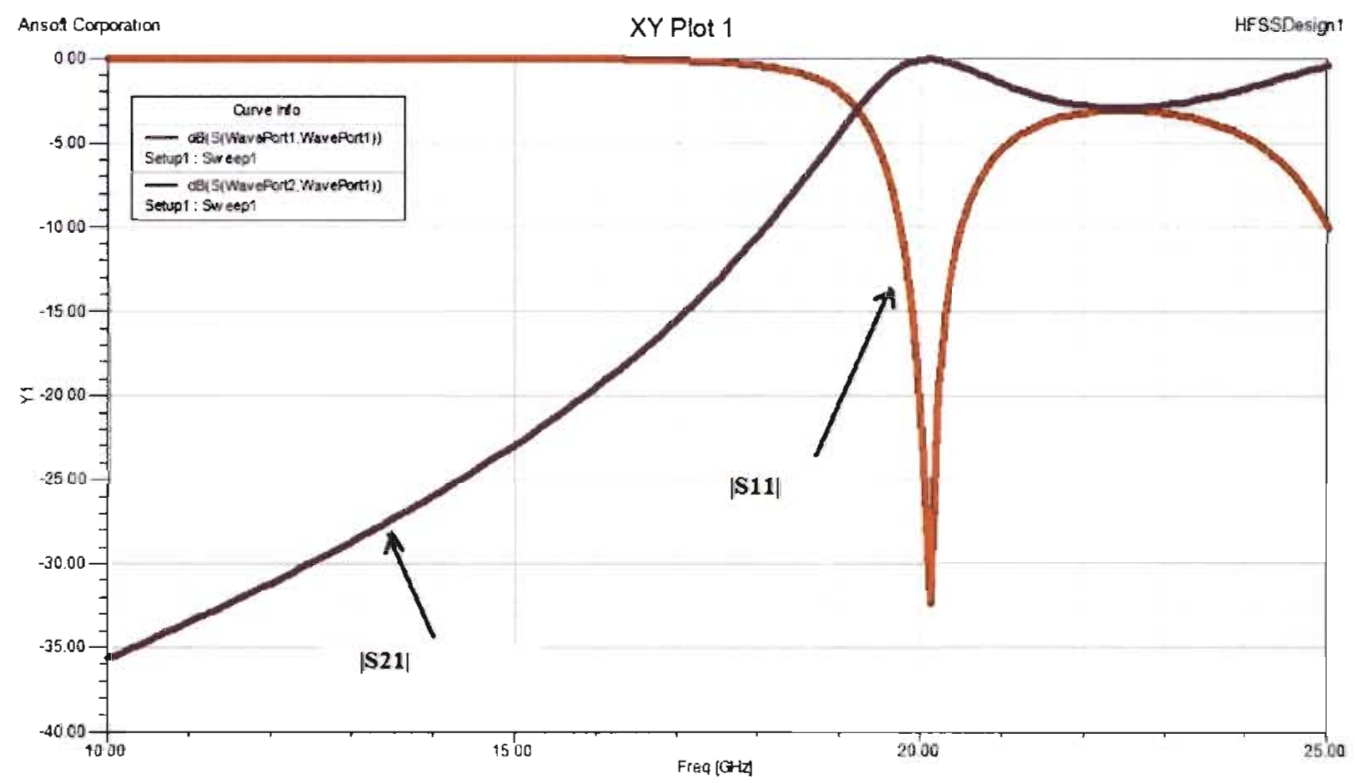

Figure 4.2 Transmission results from a $3 \times 3$ wire rod array simulated in HFSS. Cylinder radius $=0.2 \mathrm{~mm}$. Lattice spacing, $a=5 \mathrm{~mm}$, and the dielectric medium is air. Resonance is seen at $20 \mathrm{GHz}$. The location of the plasma frequency is thus at $\omega_{p}=20 \mathrm{GHz}$.

Reducing the wire width has the effect of reducing the plasma frequency. For left handed media, this is not desired as one must be sure the entire frequency range of double negative operation desired is below the wire inclusions plasma frequency to ensure permittivity remains negative. If a low plasma frequency is desired, then one may embed the rods in a high permittivity dielectric medium in comparison to air $\left(\epsilon_{r} \approx 1\right)$. This method may be necessary when trying to design zeroindex metamaterial structures at X-band or lower frequency bands. The dielectric substrate may be used to lower the resonant plasma frequency without altering the inclusion structure. 


\subsection{Capacitively loaded strips (CLS)}

The capacitively loaded strip is an alternative inclusion that reacts to an incident E-field parallel to the plane of the inclusion. This structure uses capacitively loaded wires on each end as a replacement for the configuration of a thin wire or rod, which must be connected directly to a parallel plate to induce a dielectric response to the E-field. However, for a capacitively loaded strip, such a connection is not necessary due to the diploles loaded on the strip. Hence, the capacitive dipole strips act as parallel plates and thus the metamaterial does not require a parallel plate waveguide configuration. However, it should be noted that the capacitively loaded strip does not exhibit negative permittivity over the entire frequency range below the plasma frequency. There is a resonance in the permittivity that occurs below the plasma frequency. This is reflected in the transmission and reflection data obtained from HFSS by looking for resonance peaks in $S_{21}$. Two resonances are visible separated by a stop band where evanescent modes dominate. The first resonance corresponds to the permittivity $(\epsilon)$ becoming negative and the plasma resonance indicates the frequency range where the permittivity approaches zero. It is important that the chosen frequency for left-handed operation falls within the range of left-handed operation; where the permittivity is negative. For epsilon near zero (ENZ) metamaterials, design optimization should target the plasma frequency to be in the range of the required operation frequency. 


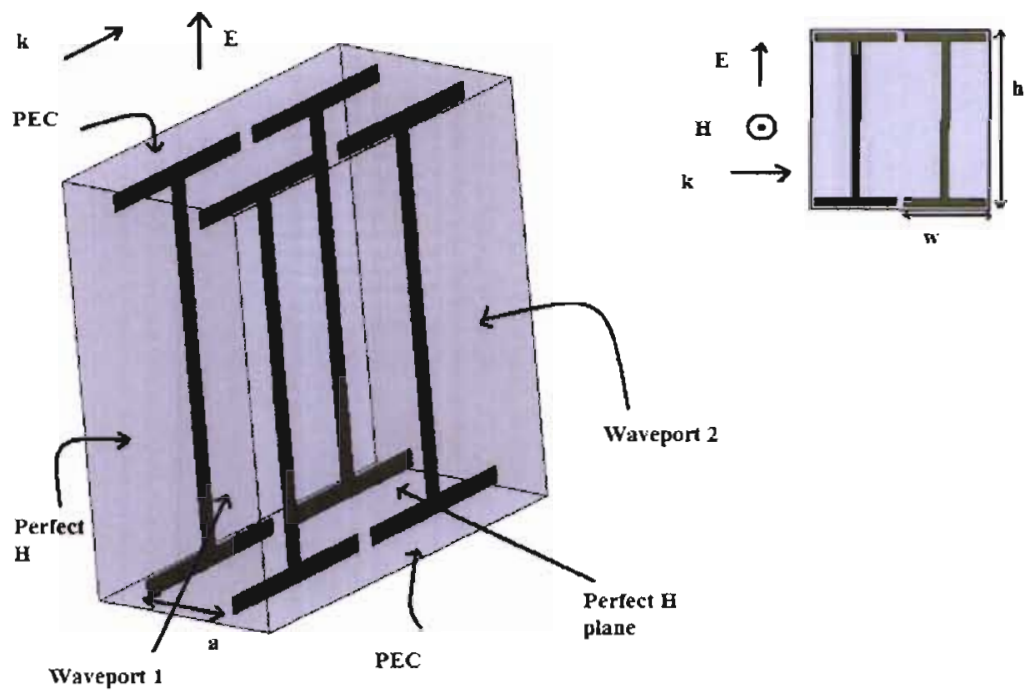

Figure 4.3 Unit cell consisting of $2 \times 2$ array of capacitive loaded strips in PEC/PMC waveguide configuration.

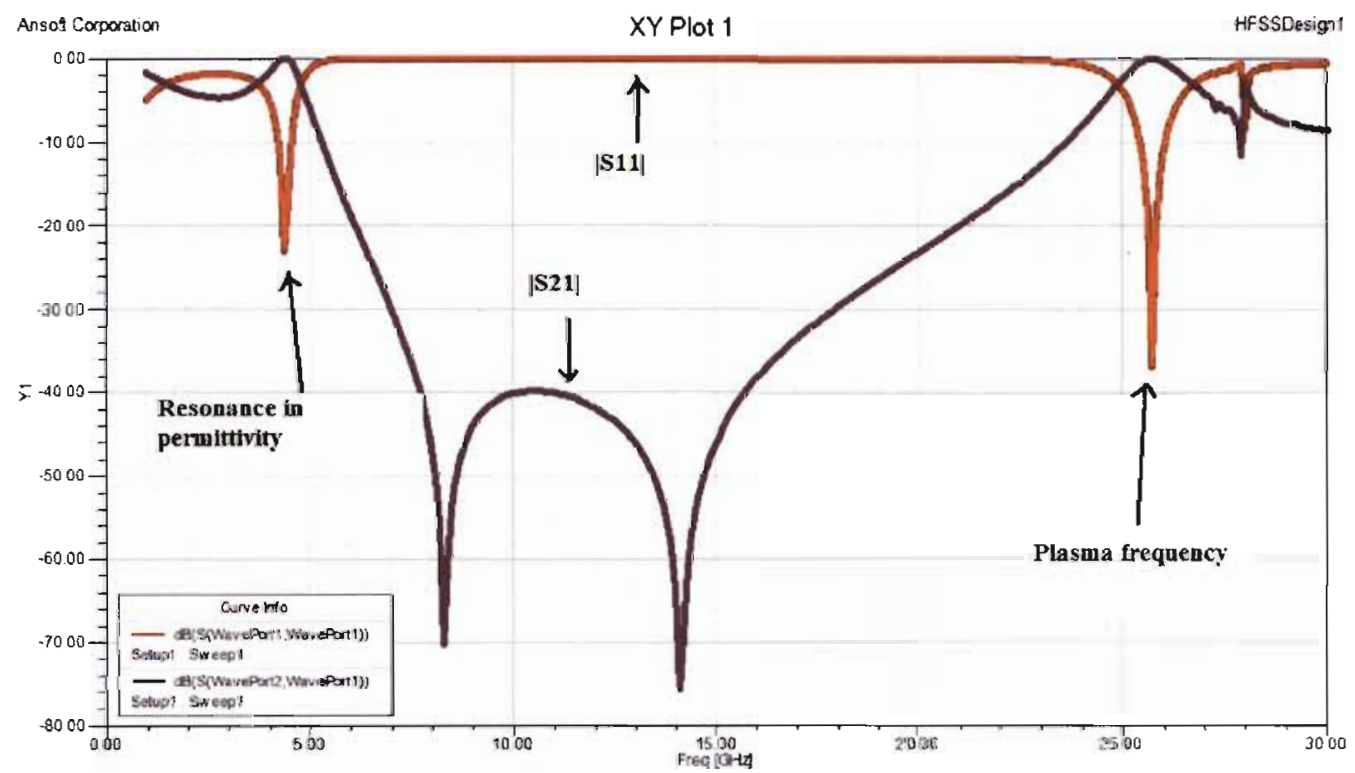

Figure 4.4 Simulated S-parameter data on a $2 \times 2$ array of CLS structures. The low resonance is at $4.4 \mathrm{Ghz}$ and the plasma frequency where evanescent modes no longer dominate is $25.7 \mathrm{GHz}$. CLS height $(\mathrm{h})=9.6 \mathrm{~mm}$ and capacitive strip width $(\mathrm{w})=4.5 \mathrm{~mm}$. Microstrip width $=0.4 \mathrm{~mm}$. Lattice spacing $(\mathrm{a})=2.5 \mathrm{~mm}$. 
The length of the capacitive loaded ends of the strips $(w)$ influences the frequency at which the permittivity of the structure goes negative. Longer strips reduce this resonance frequency as capacitive coupling between any 2 CLS structures in the H-plane will increase [31]. For testing in the $\mathrm{X}$-band frequency range, the widths must be large enough such that the first resonance occurs at a frequency lower than $7 \mathrm{GHz}$. In addition, this width is dependent on the dielectric substrate and the microstrip width. Table 4.2 shows simulated values for permittivity resonance and plasma frequency from transmission data on a $2 \times 2$ copper array of capacitively loaded strips embedded in an air PEC/PMC waveguide. The heights and lattice spacings are kept constant. Note that the plasma frequency also decreases with an increase in CLS width. However the percentage change is only $4 \%$ in comparison to the decrease in the resonant frequency of $42 \%$. Thus the resonant frequency where permittivity changes from positive to negative is solely affected by the increase in capacitor end width.

\begin{tabular}{||c|c|c||}
\hline $\begin{array}{c}\text { capacitor end width (w) } \\
\mathrm{mm}\end{array}$ & $\begin{array}{c}\text { resonant frequency }\left(f_{0}\right) \\
\mathrm{GHz}\end{array}$ & $\begin{array}{c}\text { plasma frequency } \\
\mathrm{GHz}\end{array}$ \\
\hline 0.4 & 7.7 & 26.9 \\
\hline 2 & 6.1 & 26.5 \\
\hline 2.5 & 5.6 & 26.3 \\
\hline 3 & 5.2 & 26.3 \\
\hline 4 & 4.5 & 26.1 \\
\hline 4.5 & 4.4 & 25.7 \\
\hline
\end{tabular}

Table 4.2 Simulated resonant and plasma frequencies for a $2 \times 2$ array of CLS structures in air with lattice spacing, $a=2.5 \mathrm{~mm}$, height $=9.6 \mathrm{~mm}$ and microstrip width $=0.4 \mathrm{~mm}$. The capacitive load widths on each end are varied. 


\section{Chapter 5}

\section{The S-shaped Split Ring Resonator Inclusion (S-SRR)}

An S-shaped split ring resonator has been proposed in [30]. A theoretical study of its permeabiilty and permittivity characteristics is provided in [31]. These studies are summarized here and are later used as an initial benchmark to create a periodic metamaterial slab for transmission testing. To estimate permittivity and permeability characteristics of an S-shaped resonator design prior to simulation in Ansoft HFSS, a Matlab script implementing the analytical relations developed here is implemented and can be found in the Appendix.

Circular or quadrilateral split ring resonators require the use of a rod, thin wire or capactively loaded strip structure in order to respond to the electric field and create left-handed properties at the frequency of interest. While, a circular or quadrilateral split ring resonator may respond to the electric field, the vertical asymptote (divergence) frequency for the permittivity relation is higher than that of the permeability by factors of two or three, hence the region of interest is not a true left handed metamaterial without the secondary structures [31]. The alternative resonator design proposed by Chen et al does not require secondary structures such as rods, CLSs, or thin wires.

The basic S-SRR structure along with a periodic array of such structures is shown below. The copper/metallic strips form a ' $S$ ' shape. Capacitive coupling is achieved through the addition of a 
reversed S-shaped strip printed back to back with a separation $d$.

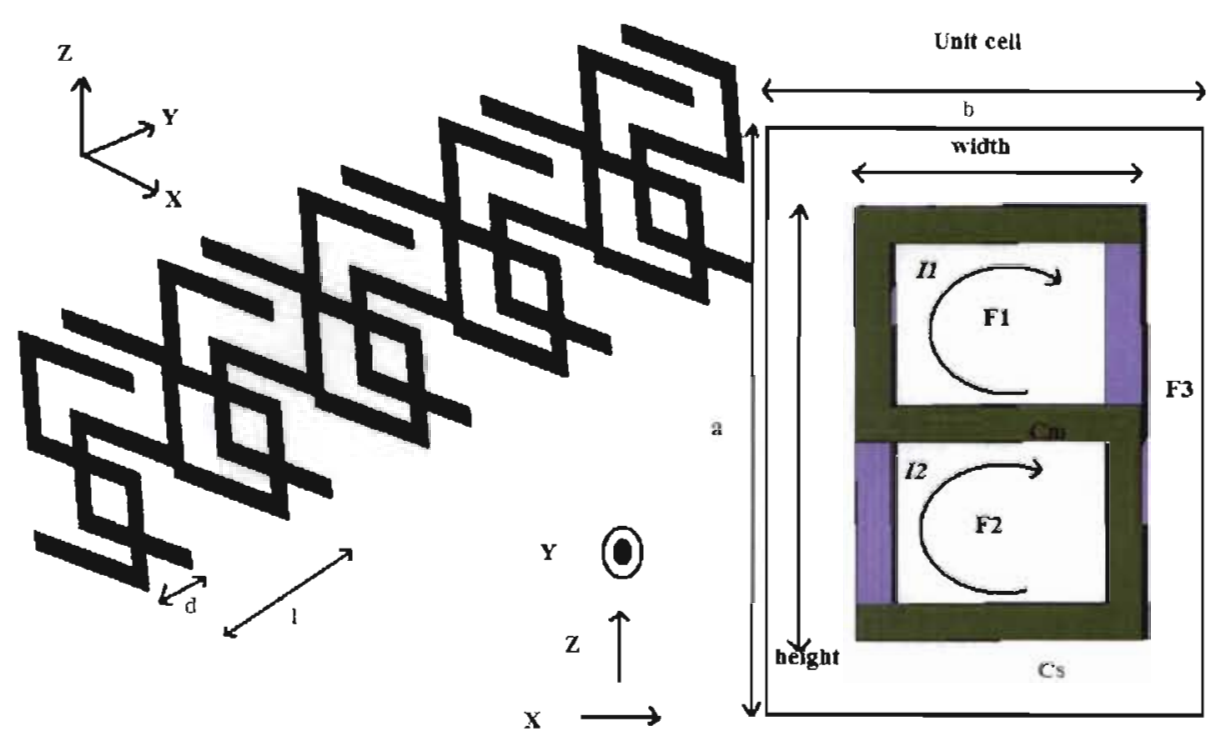

Figure 5.1 2D and 3D diagrams of S-shaped SRR structure.

The area of a periodic unit cell $\mathrm{A}$, is given by $A=x y$. The numeric ' 8 ' shaped pattern formed by the back to back split rings is shown in Figure 5.1. Three areas can be distinguished from the two dimensional image. Area I forms loop 1 at the top of the ' 8 ' pattern, while area II represents loop 2 and the bottom of the loop pattern. Area III represents the area not enclosed by these rings. Additionally, F1, F2 and F3 represent the fractional volume of the unit cell occupied by these loops. The summation of these fractional volumes is normalized to 1 :

$$
F 1+F 2+F 3=1
$$

A linearly polarized plane wave incident on the period S-SRR structures parallel to the YZ plane causes a time varying external magnetic field to be applied normal to the S-SRR plane (XZ plane). Currents flow in the split ring and are shown in the 2 dimensional diagram above. Using current 
densities (Amperes per unit length) and loop equations, the following relations must be satisfied:

$$
\begin{aligned}
j_{1} & =\frac{I_{1}}{l} \\
j_{2} & =\frac{I_{2}}{l} \\
H_{1}-H_{2} & =j_{1}-j_{2} \\
H_{1}-H_{3} & =j_{1} \\
H_{0} & =H_{1} F_{1}+H_{2} F_{2} H_{3} F_{3}
\end{aligned}
$$

Magnetic fields commonly show a spreading of the magnetic field lines. This phenomenon known as the fringing effect can be negated under the assumption that the S-SRRs along the plane they lie are sufficiently close together (a fraction of the unit cell size) Common values at X-band frequencies range from $0.1 \mathrm{~mm}$ to $2 \mathrm{~mm}$. Simulations and practical implementations shown in section 5.1 later will attempt spacing at 62 mils; a mere $1.5748 \mathrm{~mm}$. Re-arranging the above relations, it is found that $H_{2}=H_{1}-j_{1}+j_{2}$ and $H_{3}=H_{1}-j_{1}$. Hence, $H_{2}$ and $H_{3}$ may be substituted into (5.2) in terms of $H_{1}$ :

$$
\begin{aligned}
& H_{0}=H_{1} F_{1}+\left(H_{1}-j_{1}+j_{2}\right) F_{2}+\left(H_{1}-j_{1}\right) F_{3} \\
& H_{0}=H_{1} F_{1}+H_{1} F_{2}-j_{1} F_{2}+j_{2} F_{2}+H_{1}-H_{1} F_{1}-H_{1} F_{2}-j_{1}+F_{1} j_{1}+F_{2} j_{1} \\
& H_{1}=H_{0}+j_{1}-j_{2} F_{2}-j_{1} F_{1}
\end{aligned}
$$

This analysis is repeated for $H_{2}$ and $H_{3}$ resulting in three equations for the time varying magnetic fields in each region of interest:

$$
\begin{aligned}
& H_{1}=H_{0}+\left(1-F_{1}\right) j_{1}-F_{2} j_{2} \\
& H_{2}=H_{0}-F_{1} j_{1}+\left(1-F_{2}\right) j_{2} \\
& H_{3}=H_{0}-F_{1} j_{1}-F_{2} j_{2}
\end{aligned}
$$

As there is no voltage potential related with the S-SRR structures, the net electromotive force around both loops sums to zero. It should be noted that the fractional total area used to determine 
potential is $F_{1} S$ for loop 1, where $S$ has previously been described as the total area of the periodic unit cell [31].

$$
\begin{aligned}
e m f_{1} & =-\frac{\partial}{\partial t}\left(\mu_{0} H_{1} F_{1} S\right) \\
& =R_{s} I_{1}+\frac{1}{C_{s}} \int I_{1} d t+\frac{1}{C_{m}} \int\left(I_{1}+I_{2}\right) d t \\
e m f_{2} & =-\frac{\partial}{\partial t}\left(\mu_{0} H_{2} F_{2} S\right) \\
& =R_{s} I_{2}+\frac{1}{C_{s}} \int I_{2} d t+\frac{1}{C_{m}} \int\left(I_{1}+I_{2}\right) d t
\end{aligned}
$$

$R_{s}$ represents the resistances of the metallic strips. Capacitances $C_{s}$ and $C_{m}$ are due to the top/bottom metallic strips and the mutual capacitance of the center metallic strips respectively. The right-hand integral of (5.4) and (5.5) has a sum of currents $I 1+I 2$ due to the loop arrangement. The capacitance between the two metallic strips is calculated assuming the distance between the S-SRR layer and the inverted layer remains constant throughout. Thus $C_{s}$ and $C_{m}$ are equal:

$$
C_{s}=C_{m}=\epsilon_{0} \frac{h c}{d}+\epsilon_{0} \frac{h c}{l-d}
$$

To determine the frequency dependence of permeability in the S-SRR structure, (5.4) and (5.5) must be represented in the frequency domain. The summation of operations in the time domain remains as such in the frequency domain. The partial derivative operation $\frac{\partial}{\partial t}$ may be represented by $-i \omega$ and the integral $\int d t$ by $-\frac{1}{i \omega}$ in the frequency domain.

$$
\begin{gathered}
i \omega \mu_{0} H_{1} F_{1} S=R_{s} j_{1} l-\frac{1}{i C_{s} \omega} j_{1} l-\frac{1}{C_{m} \omega}\left(j_{1}+j_{2}\right) l \\
i \omega \mu_{0} H_{2} F_{2} S=R_{s} j_{2} l-\frac{1}{i C_{s} \omega} j_{2} l-\frac{1}{C_{m} \omega}\left(j_{1}+j_{2}\right) l \\
i \omega \mu_{0} H_{1} F_{1} S-R_{s} j_{1} l+\frac{1}{i C_{s} \omega} j_{1} l+\frac{1}{C_{m} \omega}\left(j_{1}+j_{2}\right) l=0 \\
i \omega \mu_{0} H_{2} F_{2} S-R_{s} j_{2} l+\frac{1}{i C_{s} \omega} j_{2} l+\frac{1}{C_{m} \omega}\left(j_{1}+j_{2}\right) l=0
\end{gathered}
$$


From Pendry's analysis of the circular SRR structure in [17], the permeability can be defined in terms of the time varying magnetic field and the permeability of free space:

$$
\mu_{e f f}=\frac{B_{0}}{\mu_{0} H_{3}}=\frac{H_{0}}{H_{0}-F_{1} j_{1}-F_{2} j_{2}}
$$

The current densities $j_{1}$ and $j_{2}$ are unknown and must be substituted out. This is done through the re-arrangement and substitution of (5.7) into (5.8). The result of the lengthy calculation is shown by Chen et al to produce a complex relation for the effective permeability:

$$
\mu_{c f f}=1-\frac{\left(\omega \mu_{0} S\right)^{2} F_{1} F_{2}\left(F_{1}+F_{2}\right)-\mu_{0} S\left[\left(F_{1}^{2}+F_{2}^{2}\right) \frac{1}{C_{s}}+\left(F_{1}-F_{2}\right)^{2} \frac{1}{C_{m}}\right]+i A(R)}{\left(\omega \mu_{0} S\right)^{2} F_{1} F_{2}-\mu_{0} S(F 1+F 2)\left(\frac{l}{C_{s}}+\frac{l}{C_{m}}\right)+\frac{1}{\omega^{2}} \frac{l}{C_{s}}\left(\frac{l}{C_{s}}+\frac{2 l}{C_{m}}\right)-B(R)+i C(R)}
$$

The following abbreviations are used:

$$
\begin{aligned}
& A(R)=\omega \mu_{0} S\left(F_{1}^{2}+F_{2}^{2}\right)+R_{s} l \\
& B(R)=\left(R_{s} l\right)^{2} \\
& C(R)=\left[\omega \mu_{0} S(F 1+F 2)-\frac{2}{\omega}\left(\frac{l}{C_{s}} \frac{l}{C_{m}}\right)\right] R_{s} l
\end{aligned}
$$

The divergence or vertical asymptotes in the effective permeability relation where the permeability becomes negative can be found by finding the frequency, $\omega$, where the denominator equals zero. Due to the quadratic dependency in the denominator, there are 2 solutions. Hence, in general, if the loop areas $F_{1}$ and $F_{2}$ are not equal, then equation (5.10) has two resonant frequencies:

$$
\begin{aligned}
& \omega_{m 01}=\sqrt{\frac{\left(\frac{F_{1}}{F_{2}}+1\right)\left(\frac{C_{m}}{C_{s}}+1\right)+\sqrt{\left(\frac{F_{1}}{F_{2}}-1\right)^{2}\left(\left(\frac{C_{m}}{C_{s}}\right)^{2}+2 n\right)+\left(\frac{F_{1}}{F_{2}}+1\right)^{2}}}{2 \frac{F_{1}}{F_{2}} \frac{C_{m}}{C_{s}}} \frac{l}{\mu_{0} S F_{2} C_{s}}} \\
& \omega_{m 02}=\sqrt{\frac{\left(\frac{F_{1}}{F_{2}}+1\right)\left(\frac{C_{m}}{C_{s}}+1\right)-\sqrt{\left(\frac{F_{1}}{F_{2}}-1\right)^{2}\left(\left(\frac{C_{m}}{C_{s}}\right)^{2}+2 n\right)+\left(\frac{F_{1}}{F_{2}}+1\right)^{2}}}{2 \frac{F_{1}}{F_{2}} \frac{C_{m}}{C_{s}}} \frac{l}{\mu_{0} S F_{2} C_{s}}}
\end{aligned}
$$


When $F_{1}=F_{2}=F$, there is only one resonant frequency related to the effective permeability. The permeabiliy relation and the resonant frequency solution may be represented as:

$$
\mu_{e f f}=1-\frac{2 F+i D}{1-\frac{1}{\omega^{2} \mu_{0} F S}\left(\frac{l}{C_{s}}+\frac{2 l}{C_{m}}\right)-E+i G}
$$

where

$$
\begin{aligned}
X & =\left(\omega \mu_{0} F S\right)^{2}\left(1-\frac{1}{\omega \mu_{0} F S} \frac{l}{C_{s}}\right) \\
D & =\frac{A}{X} \\
E & =\frac{B}{X} \\
G & =\frac{C}{X}
\end{aligned}
$$

$$
\omega_{m 0}=\sqrt{\frac{1}{\mu_{0} F S}\left(\frac{l}{C_{s}}+\frac{2 l}{C_{m}}\right)}
$$

Prior to simulation it is important to first choose the desired resonance frequency(s) and obtain the inclusion dimensions that realize the theoretical desired resonance frequency goals.

\subsection{S-shaped SRR inclusion simulations}

\subsubsection{Unit cell Synthesis}

Like the split ring resonator structures synthesized in HFSS, the S-SRR structures may also be modeled with a PEC/PMC waveguide model. One s-shaped copper structure may be embedded in a dielectric medium. To generate the mirrored $\mathrm{S}$ shape, the mirror and move commands are used. Again modal S-parameters are generated using 2 waveports with one dominant mode as described by the integration line.

\subsubsection{Simulation results}

As in simulations for the basic SRR structures presented in the previous chapter, simulations in HFSS are performed to verify analytical equations such as $(5.12-5.13,5.15)$. The table below depicts 


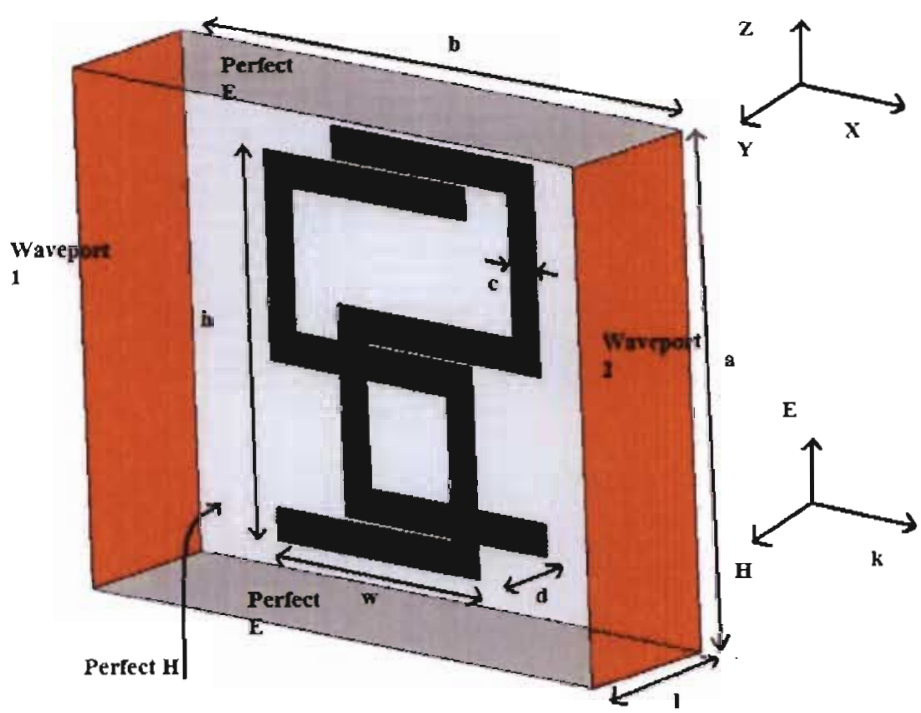

Figure 5.2 A synthesized S-SRR unit cell in HFSS. Copper thickness is 1 oz $(0.2 \mathrm{~mm})$. $F_{1}=F_{2}$ in this case.

the theoretical and simulated resonant frequencies for varying unit cells. Note that the analytic equations described above may provide methods to obtain 2 resonant frequencies due to the differing current loop dimensions in the S-shaped SRR inclusions. However, Table 5.1 presents results for unit cell designs with a single established resonant frequency. The two loops formed by the mirrored $S$-shapes are thus identical and $F_{1}=F_{2}$.

\begin{tabular}{||c|c|c|c|c|c|c|c|c|c||}
\hline $\begin{array}{c}\mathrm{w} \\
(\mathrm{mm})\end{array}$ & $\begin{array}{c}\mathrm{h} \\
(\mathrm{mm})\end{array}$ & $\begin{array}{c}\mathrm{a} \\
(\mathrm{mm})\end{array}$ & $\begin{array}{c}\mathrm{b} \\
(\mathrm{mm})\end{array}$ & $\begin{array}{c}\mathrm{c} \\
(\mathrm{mm})\end{array}$ & $\begin{array}{c}\mathrm{d} \\
(\mathrm{mm})\end{array}$ & $\begin{array}{c}\mathrm{l} \\
(\mathrm{mm})\end{array}$ & $\begin{array}{c}\text { theoretical } \\
f_{0}(\mathrm{GHz})\end{array}$ & $\begin{array}{c}\text { Actual } \\
f_{0}(\mathrm{GHz})\end{array}$ & $\%$ diff \\
\hline 4 & 5 & 8 & 6.5 & 0.5 & 1.57 & 2.36 & 20.6 & 18.8 & 8.737 \\
\hline 3 & 7 & 9 & 7 & 0.5 & 0.9 & 2 & 17.7 & 14.5 & 18.079 \\
\hline 4.2 & 7.4 & 9 & 7.5 & 0.6 & 1.2 & 2 & 12.9 & 11 & 14.728 \\
\hline 3.9 & 5.8 & 8.5 & 7.5 & 0.75 & 0.5 & 1.8 & 11.6 & 10.3 & 11.21 \\
\hline 5 & 10 & 8 & 11 & 1 & 0.75 & 1.5 & 5.5 & 6.8 & 23.64 \\
\hline
\end{tabular}

Table 5.1 HFSS simulation results for varying unit cell dimensions. Results show the $S$-SRR equations provide only a very rough approximation and that HFSS simulations are absolutely necessary to characterize a SRR inclusion.

\subsubsection{Parameter Variations}

Unlike the Split ring resonators presented in the previous chapter, the $S$-shaped resonators are not uniform in radius. Hence, the height and the width of the structures will be treated individually here 
and may be separately optimized to reach resonance frequency goals. It is expected that increasing the inductance increases the overall inductance of the structure. An increase to bulk capacitance or inductance inherently reduces the resonant frequency. The same may be said of an increase in S-SRR width. The effect of S-SRR height is confirmed in Figure 5.3. In this simulation, SRR width $=4 \mathrm{~mm}$, copper width $=0.5 \mathrm{~mm}$, spacing between mirrored S-shaped resonators $(\mathrm{d})=1.5 \mathrm{~mm}$, distsance between $S$-shape pairs, $1=2.5 \mathrm{~mm}$. Unit cell height $(\mathrm{a})=10 \mathrm{~mm}$ and width $(\mathrm{b})=7 \mathrm{~mm}$. Similarly, the effect of width on the S-shaped split ring resonator may be seen in Figure 5.4. In this case, $S R R$ height $=6 \mathrm{~mm}$, copper width $=0.5 \mathrm{~mm}$, spacing between mirrored $S$-shaped resonators (d) $=1.5 \mathrm{~mm}$, distsance between $S$-shape pairs, $1=2.5 \mathrm{~mm}$. Unit cell height (a) $=10 \mathrm{~mm}$ and width (b) $=7 \mathrm{~mm}$.

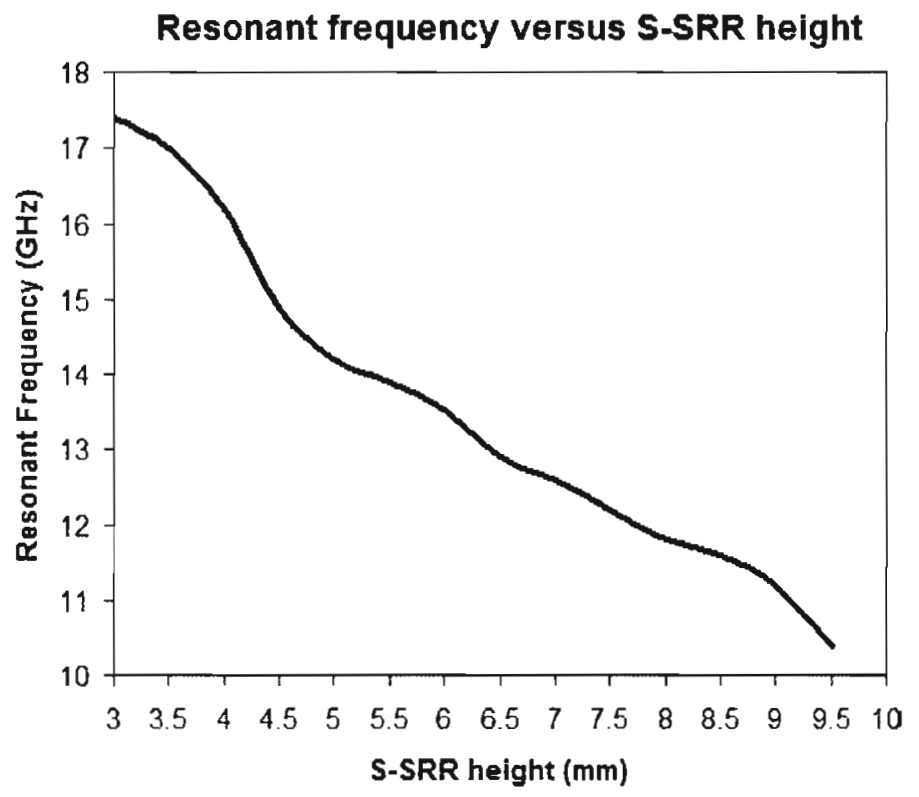

Figure 5.3 Resonant Frequency of S-SRR for varying inclusion heights.

Dielectric permittivity affects the measured resonance frequency. The simulation results show that embedding the copper inclusions in Roger's Duroid $5880\left(\epsilon_{r}=2.2\right)$ produces a resonance frequency higher than that of an inclusion embedded in FR-4 substrate $\left(\epsilon_{r}=4.4\right)$. The relation may be approximated with a linear fit for relative permittivities $>2$.

To validate the relations for uneven current loops in the S-SRR structure where $F_{1} \neq F_{2}$ (5.12) 


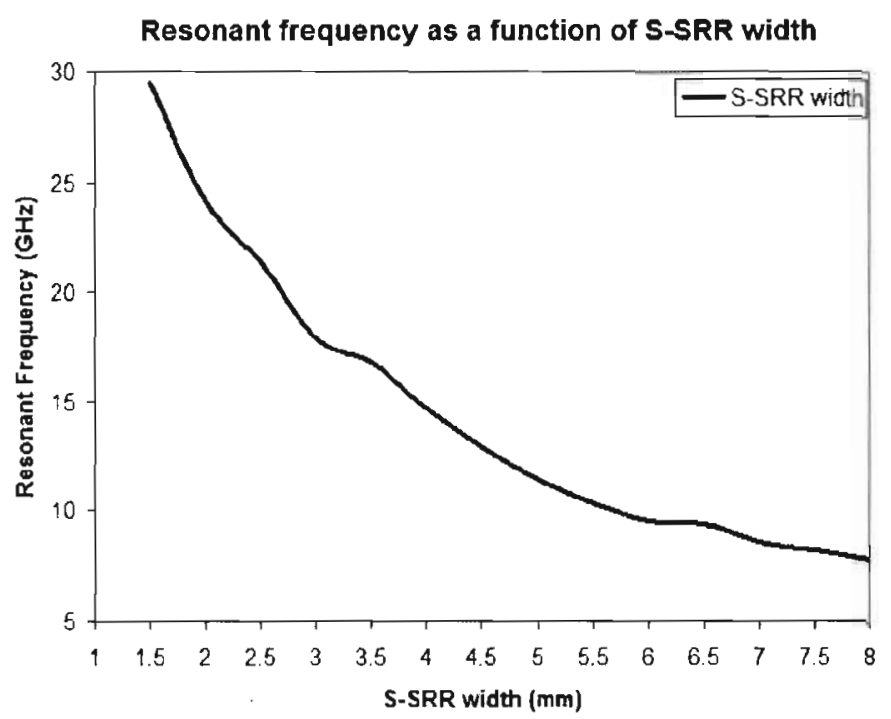

Figure 5.4 Resonant Frequency of S-SRR for varying inclusion widths.

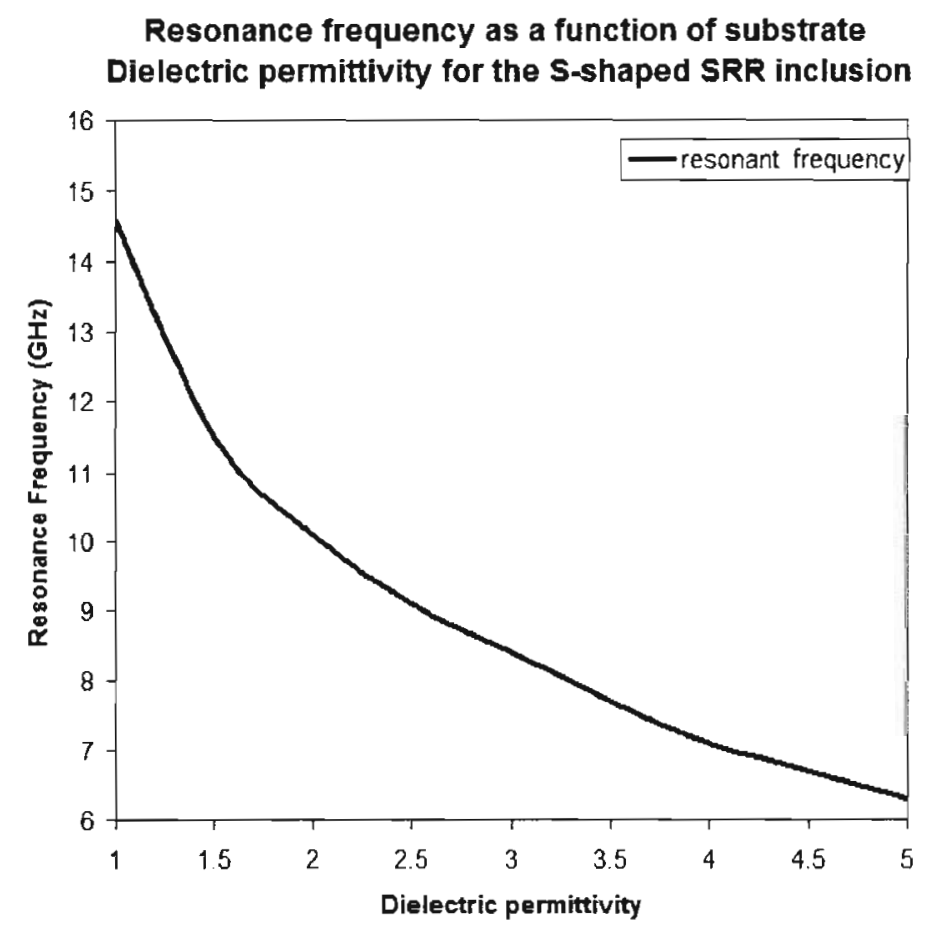

Figure 5.5 Resonant Frequency of S-SRR for varying dielectric permittivities. SRR height $=6 \mathrm{~mm}$, SRR width $=4 \mathrm{~mm}$, copper width $=0.5 \mathrm{~mm}$, spacing between mirrored S-shaped resonators $(\mathrm{d})=1.5 \mathrm{~mm}$, distsance between $S$-shape pairs, $1=2.5 \mathrm{~mm}$. Unit cell height $(\mathrm{a})=10 \mathrm{~mm}$ and width $(\mathrm{b})=7 \mathrm{~mm}$. 
and (5.13), the ratios of the areas covered by the 2 loops; $F_{1}, F_{2}$ are varied. To accomplish this in HFSS, variables are used to alter the location of the center bar of each S-shape by specifying a ratio between 0 and 1 . A value of 0 specifies the geometry in which the center bar overlaps the top bar of the ' $S$ ' shape. A value of 0.5 means it remains in the center and $F_{1}=F_{2}$. A ratio of 1 thus means the center bar now overlaps the bottom bar of the ' $S$ ' shape. Table 5.2 presents the results of variable center bar location and thus the proportion of the unit cell enclosed by each loop: $F_{1}$ and $F_{2}$

\begin{tabular}{||c|c|c|c|c|c||}
\hline$F_{1}$ & $F_{2}$ & theoretical $f_{01}$ & theoretical $f_{02}$ & simul $f_{01}$ & simul $f_{02}$ \\
\hline 0.3 & 0.3 & 7.54 & N/A & 7.3 & N/A \\
\hline 0.36 & 0.24 & 4.31 & 7.7 & 4.3 & 7.5 \\
\hline 0.42 & 0.18 & 4.2 & 8.5 & 4.1 & 7.9 \\
\hline 0.48 & 0.12 & 4.06 & 10.1 & 4.15 & 8.7 \\
\hline 0.51 & 0.09 & 3.99 & 11.5 & 3.88 & 9.3 \\
\hline
\end{tabular}

Table 5.2 HFSS simulation results for varying loop ratios to alter the two resonance frequencies.
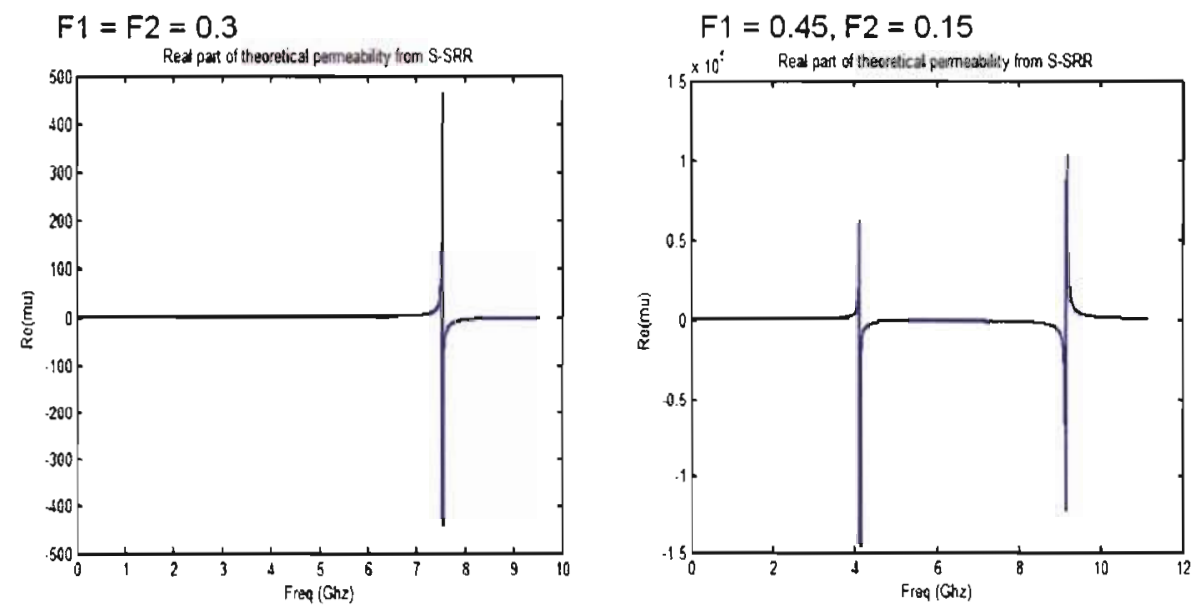

Figure 5.6 Divergence/resonance simulation in matlab for an S-SRR unit cell with height $=7.5 \mathrm{~mm}$, width $=4 \mathrm{~mm}$, copper width $=0.5 \mathrm{~mm}$, distance between mirrored rings $=0.5 \mathrm{~mm}, S-S R R$ pair seperation, $l=1 \mathrm{~mm}$, unit cell: $10 \mathrm{~mm} \times 5 \mathrm{~mm}$. 


\subsection{Summary of metallic inclusions}

\begin{tabular}{||c|c|c|c|c||}
\hline & SRR & Thin rods/ CLS & $\begin{array}{c}\text { SRR/CLS } \\
\text { combined } \\
\text { structure }\end{array}$ & S-SRR \\
\hline $\begin{array}{c}\text { Transmission } \\
\text { at resonance }\end{array}$ & $S_{21}->0$ & $S_{21}->1$ at & $\begin{array}{c}S_{21}->1 \\
\text { plasma resonance }\end{array}$ & $S_{21}->1$ \\
\hline $\begin{array}{c}\text { Effect on } \\
\text { permittivity }\end{array}$ & NONE & $\begin{array}{c}\text { Negative below the } \\
\text { plasma } \\
\text { frequency. }\end{array}$ & $\begin{array}{c}\text { Negative at the } \\
\text { resonance } \\
\text { frequency. }\end{array}$ & $\begin{array}{c}\text { Negative at the } \\
\text { resonance } \\
\text { frequency. }\end{array}$ \\
\hline Effect on \\
permeability & $\begin{array}{c}\text { Negative at the } \\
\text { resonance } \\
\text { frequency. }\end{array}$ & NONE & $\begin{array}{c}\text { Negative at the } \\
\text { resonance } \\
\text { frequency. }\end{array}$ & $\begin{array}{c}\text { Negative at the } \\
\text { resonance } \\
\text { frequency. }\end{array}$ \\
\hline
\end{tabular}

Table 5.3 Summary of the transmission, permittivity and permeability characteristics of the inclusions introduced in this thesis.

Table 5.3 indicates the common dielecric and magnetic properties seen in split ring resonator, wire strip, combined SRR/CLS and s-shaped structures. This table may be used as a summary when designing around an application. For filtering, an SRR only metamaterial would provide stop-band characteristics at the targetted resonance frequency while the combined SRR/CLS or S-shaped SRR (S-SRR) structures show pass-band/matching to free-space. 


\section{Chapter 6}

\section{Parameter Extraction applied to Discrete Metallic inclusions}

\subsection{Extraction results from HFSS simulations}

The theory for extracting homogeneous material parameters such as $\epsilon_{r}, \mu_{r}$ and $n$ is presented in Chapter 2. A Matlab script available in the Appendix reads in column vectors of data from an Excel file. This data includes frequency points measured in HFSS along with the associative scattering parameters at those frequencies. Given magnitude and phase (degrees) data for $S_{11}$ and $S_{21}$, their complex values may be obtained.

\subsubsection{Circular SRR with thin wire strips}

A unit cell composed of a circular SRR and a thin wire strip to achieve simultaneous negative permeability and permittivity. A picture of the unit cell is shown below. The transmission and reflection s-parameters are also shown as a function of frequency. 


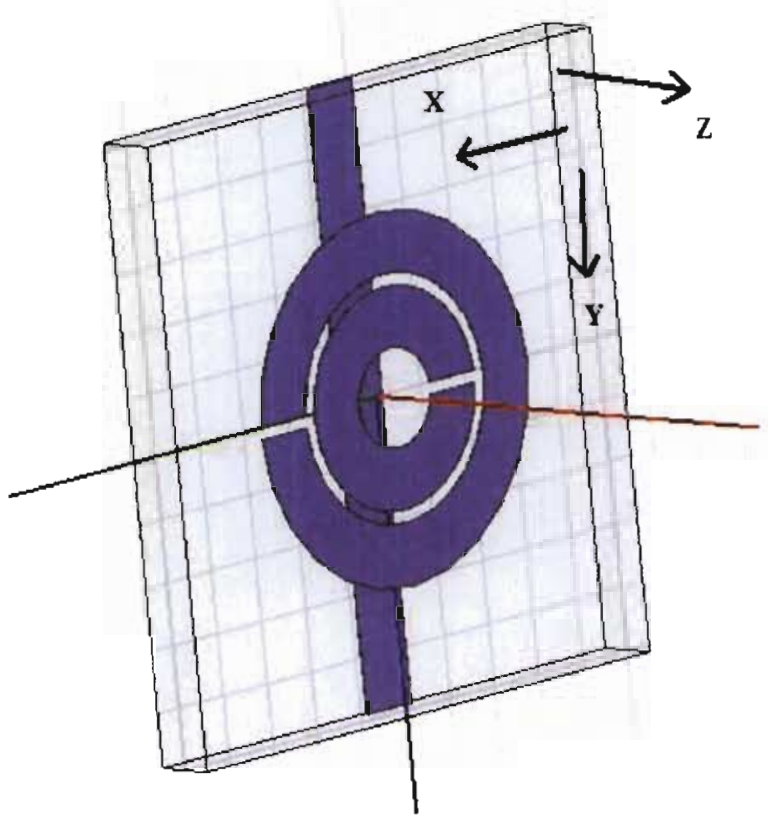

Figure 6.1 A unit cell with a circular SRR and thin wire strip. The boundaries are $10 \mathrm{~mm} x$ $10 \mathrm{~mm}$ in the $X$ and $Y$ directions. The separation between the SRR and wire strip is $1 \mathrm{~mm}$. SRR outer radius is $3 \mathrm{~mm}$ with a copper strip width of $1 \mathrm{~mm}$ and gap width of $0.2 \mathrm{~mm}$.

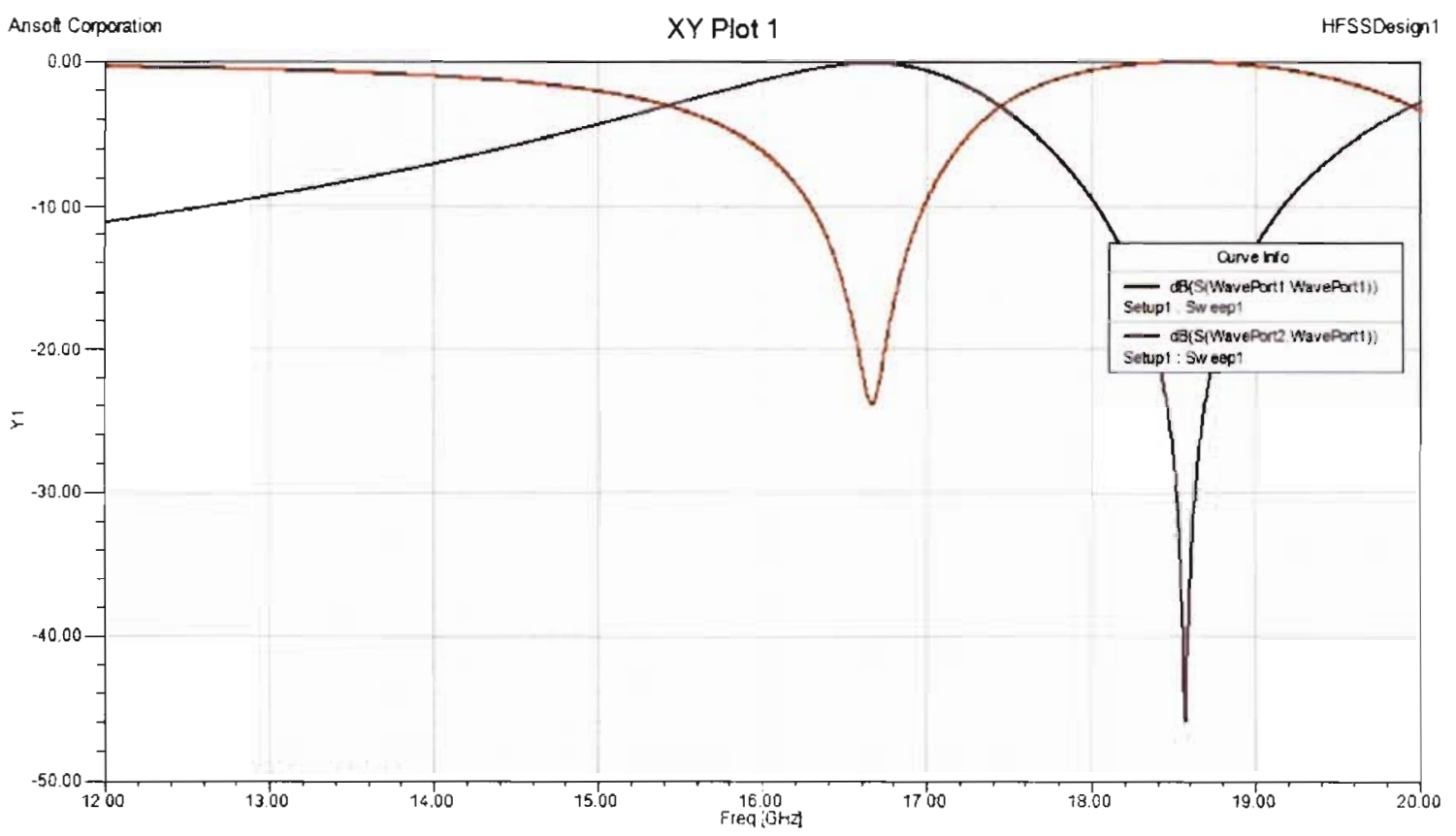

Figure 6.2 Resonance shown in scattering parameters at approximately $18 \mathrm{Ghz}$. 


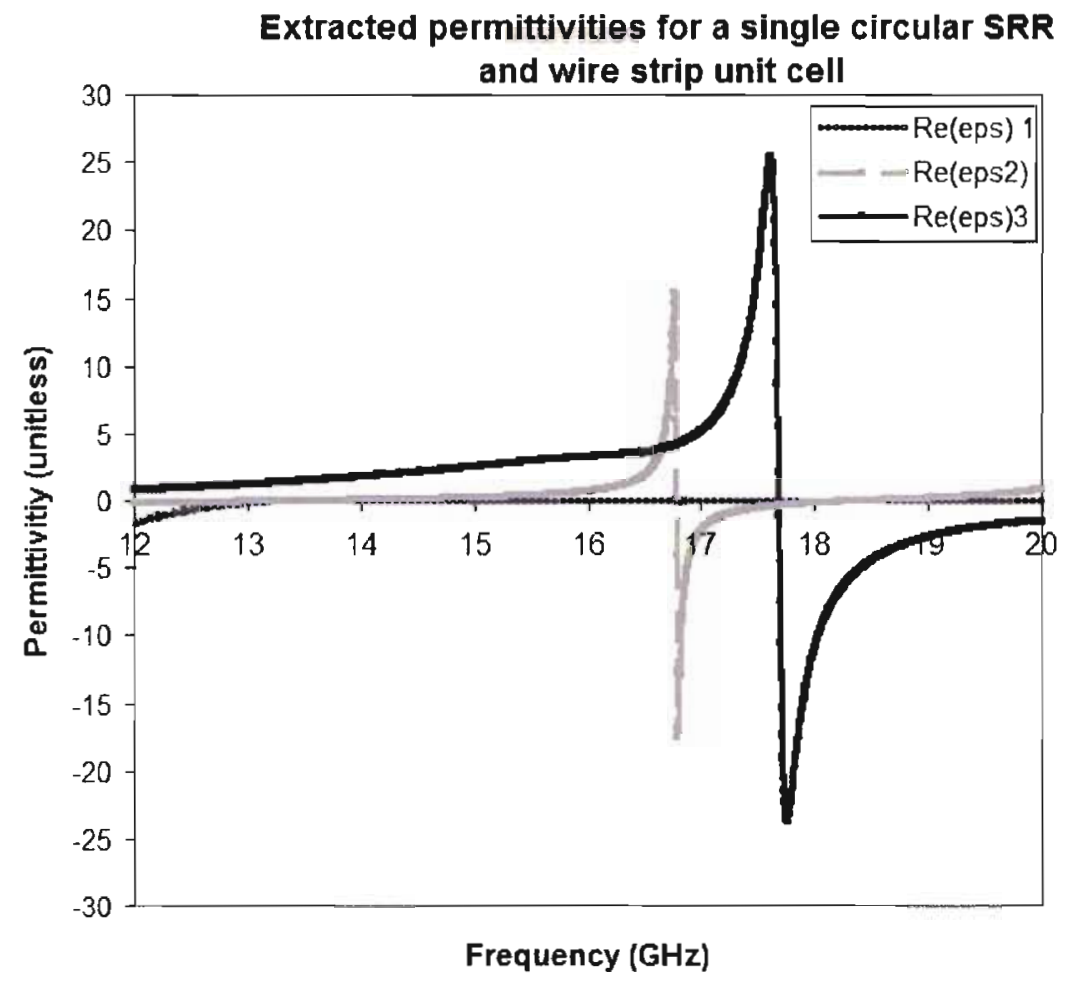

Figure 6.3 Extracted real part of $\left(\epsilon_{r}\right.$ from S-parameters. The equations are used respectively.

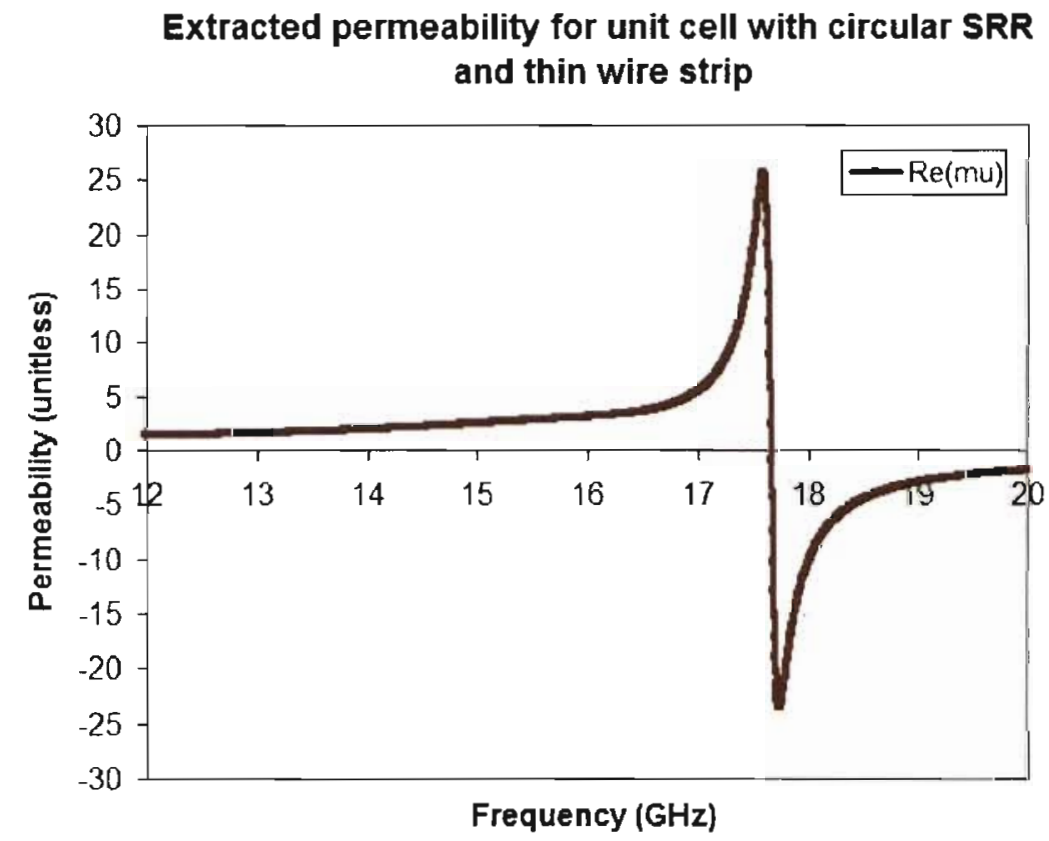

Figure 6.4 Extracted real part of $\mu_{r}$ from S-parameters. 


\section{Electrical thickness and Index of refractlon extracted from single unit cell with clrcular SRR and thin wire strip}

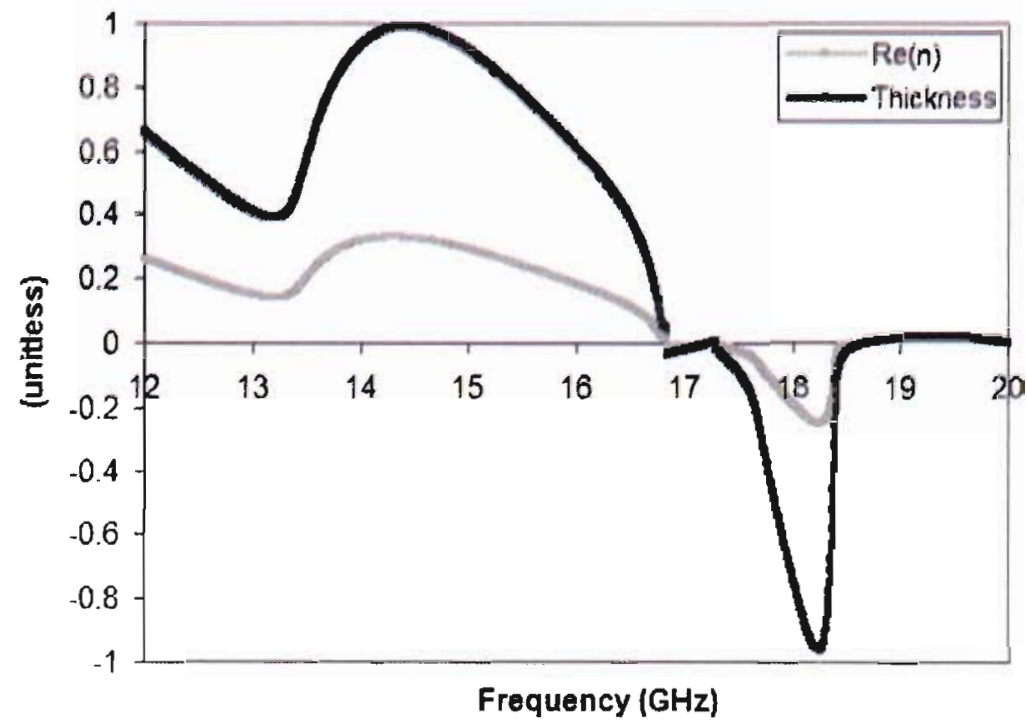

Figure 6.5 Extracted real part of the index of refraction and slab electrical thickness.

A resonance in the permeability and permittivity of the unit cell occurs at approximately 18 GHz. Several of the relations presented for $\epsilon_{r}$ in section 2.2 are used for extraction. The relation (2.29) plotted as $\operatorname{Re}(\epsilon)$ I as seen in Figure 6.3 produces subdued resonance characteristics compared to the other two relations.

\subsection{Square SRR with capacitively loaded strips}

In a similar fashion to the circular SRR and wire strip unit cell presented above, a square SRR and capacitively loaded strip in a non-planar setup is constructed in HFSS. Note that the capacitively loaded strip may be placed in the same plane as the split ring resonator. In Chapter 7 a comparison between planar and non-planar for the SRR structures is given. 


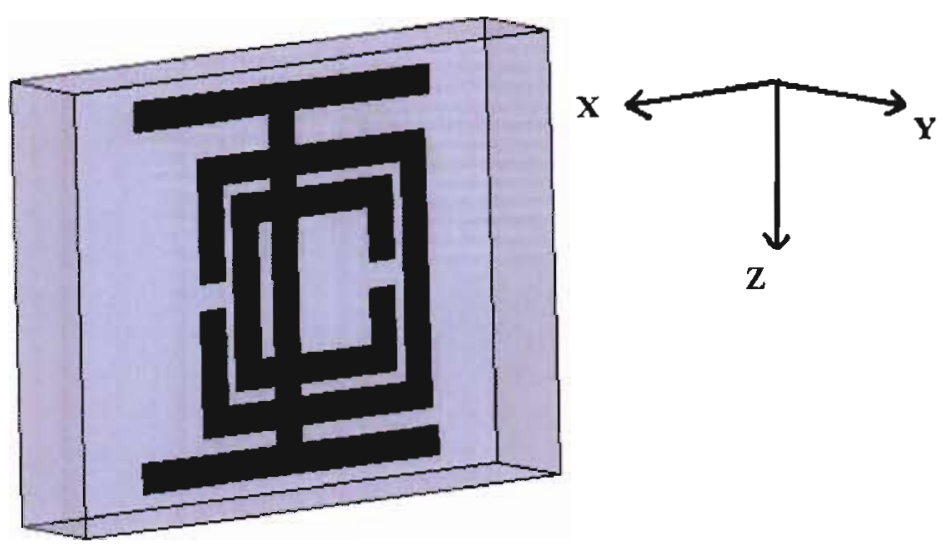

Figure 6.6 A unit cell with a square SRR and capacitively loaded strip. The boundaries are 210 mils $x 160 \mathrm{mils}$ in the $\mathrm{X}$ and $\mathrm{Y}$ directions. The separation between the Square SRR and the CLS is $0.45 \mathrm{~mm}$. SRR outer radius is 50mils with a copper strip width of $10 \mathrm{mils}$ and gap width of 5 mils. Rogers Duroid, $\epsilon_{r}=2.2$ is used as the dielectric substrate.

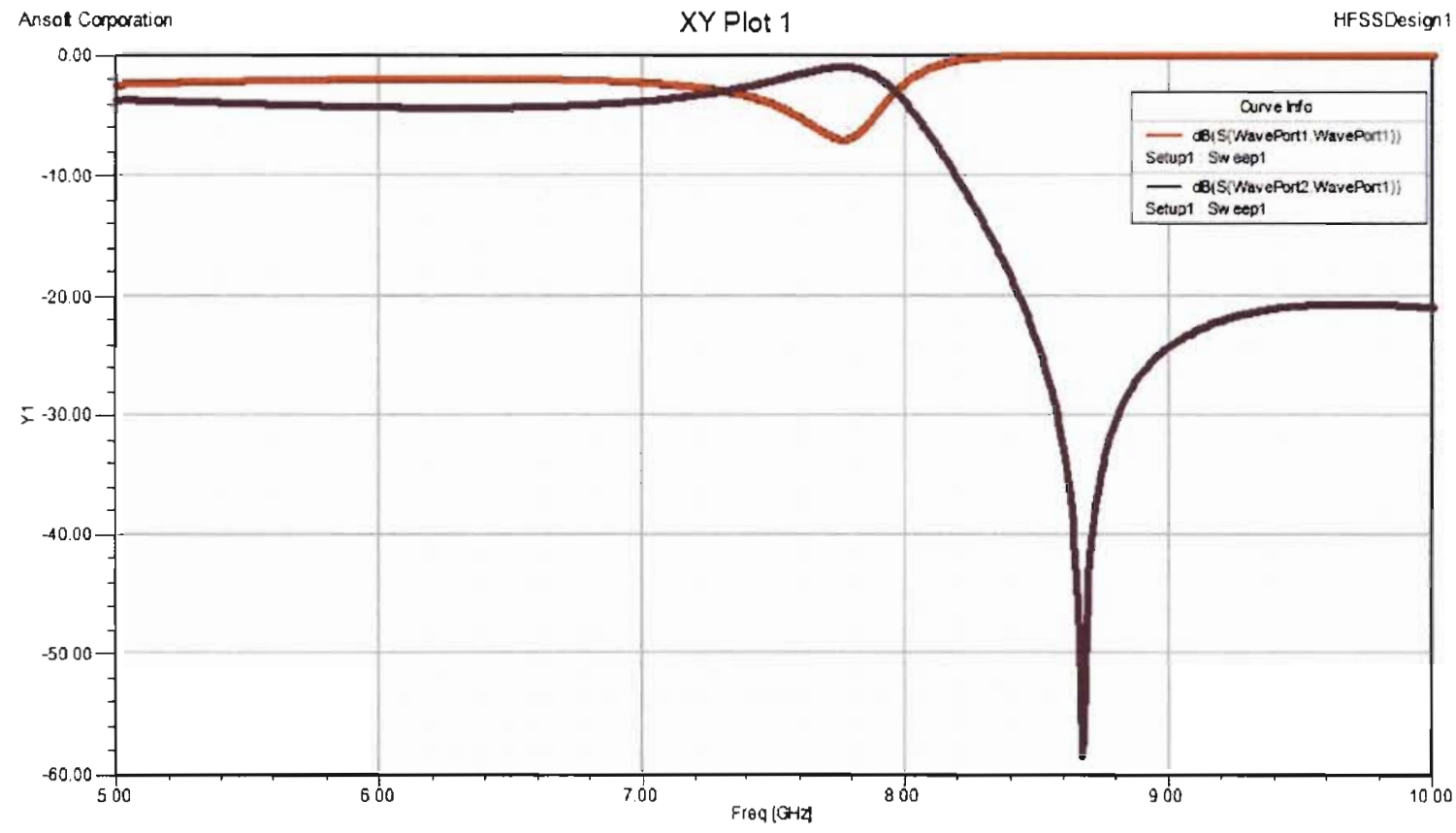

Figure 6.7 Resonance shown in scattering parameters at approximately $8 \mathrm{GHz}$. 


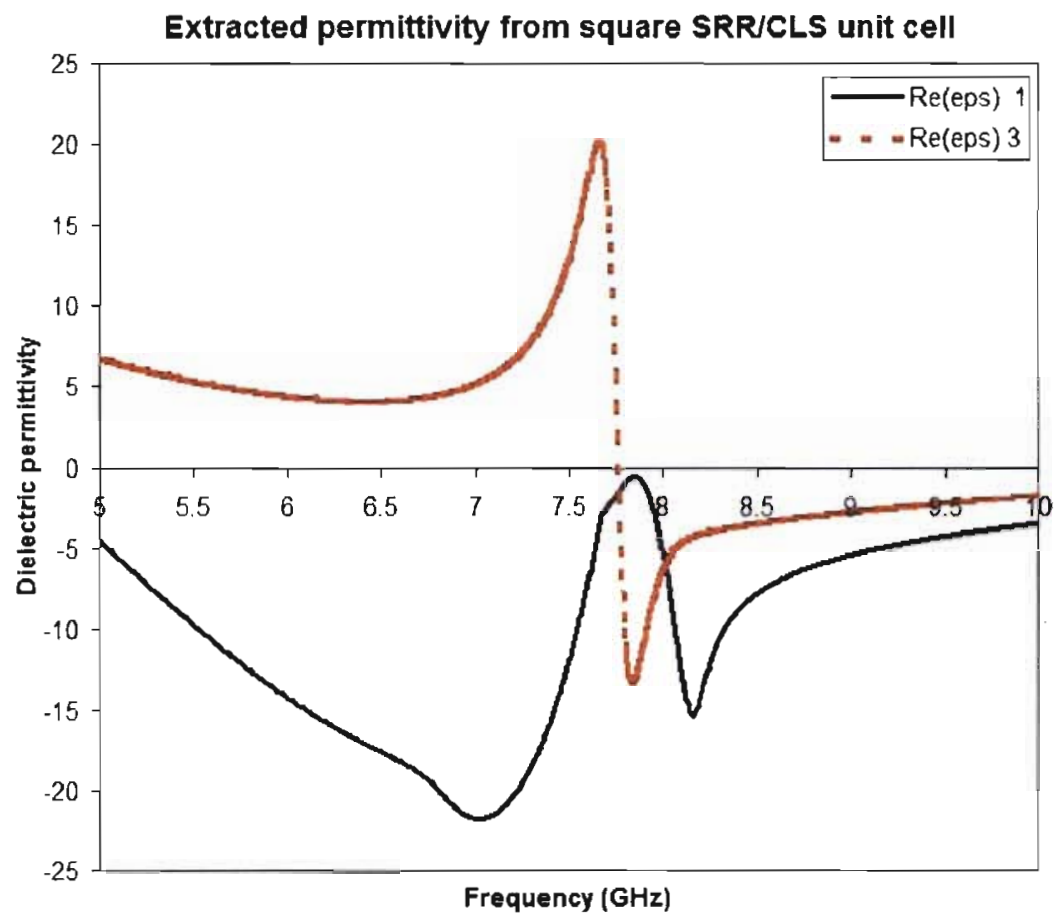

Figure 6.8 Extracted $\epsilon_{r}$ from S-parameters. The equations (2.29) (2.31) are used respectively.

Real part of the extracted permeability from the square SRR/CLS unit cell

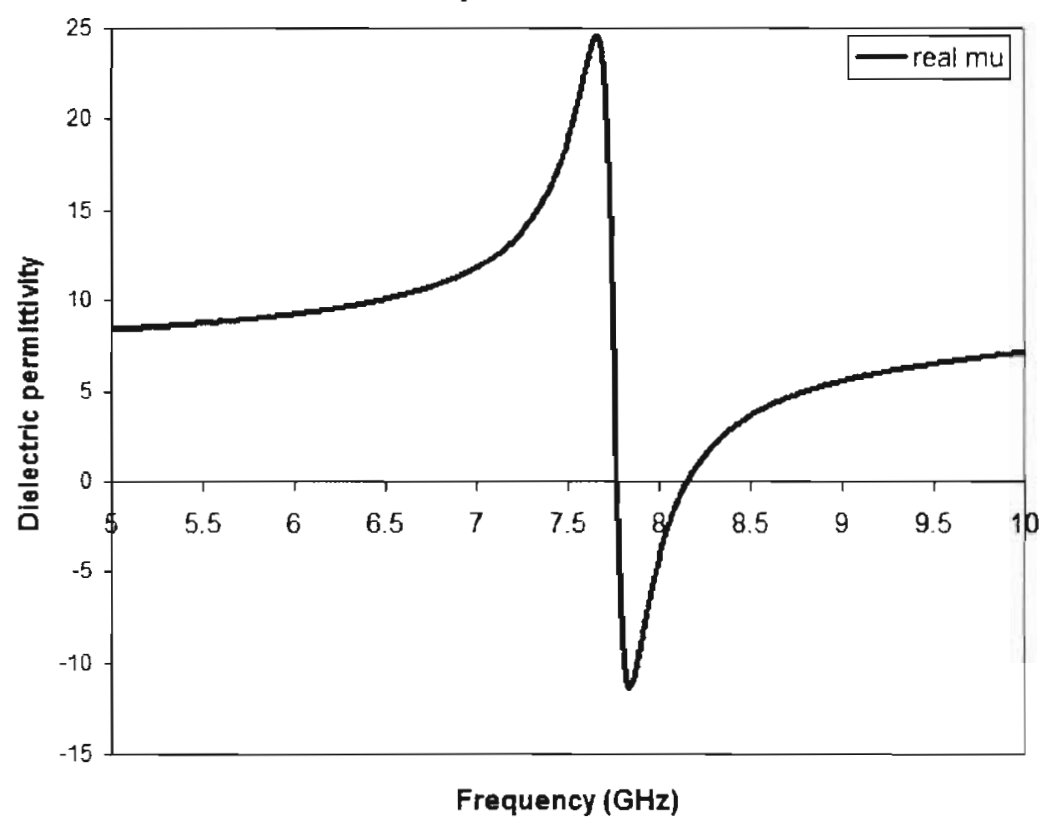

Figure 6.9 Extracted $\mu_{r}$ from $S$-parameters recorded through simulation of square SRR/CLS unit cell. 


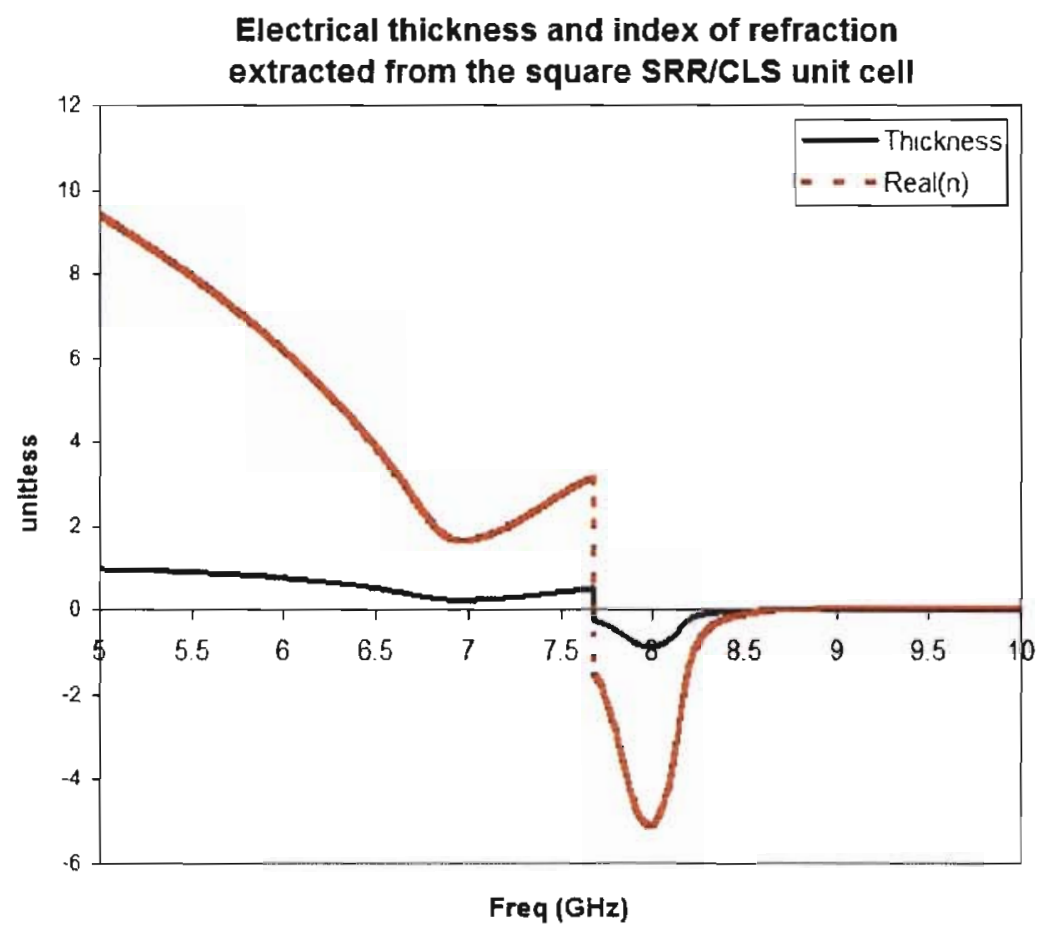

Figure 6.10 Extracted index of refraction and electrical thickness from S-parameters recorded through simulation of square SRR/CLS unit cell.

\subsection{Capacitively loaded strips}

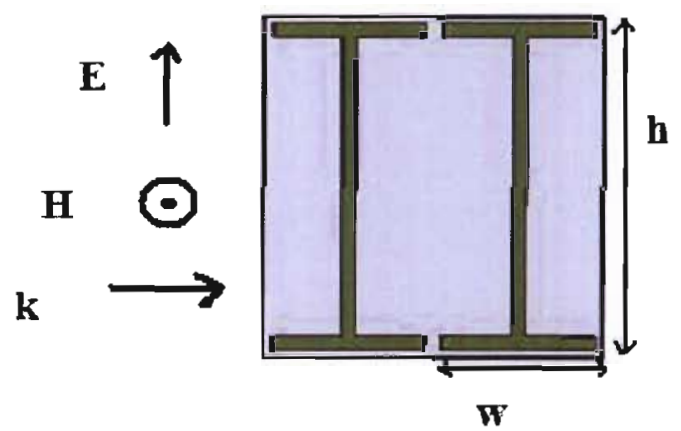

Figure 6.11 Side view of the CLS $2 \times 2$ array unit cell in HFSS. $h=15.7 \mathrm{~mm}, \mathrm{~h}=4.5 \mathrm{~mm}$, and lattice spacing $a=5 \mathrm{~mm}$. The copper inclusions are embedded in an air box.

Simulation and parameter extraction of a $2 \times 2$ array of capacitively loaded strips shows that permittivity is negative up to the expected plasma resonance frequency as seen in Figure 6.12. A resonance is seen in the permeability at this frequency as predicted by equation (2.28). The real part 
of the index of refraction must not be taken at face value. Below the plasma resonance frequency, the index of refraction is shown to be zero. In this frequency range, $\mu_{r}>0$ and $\epsilon_{r}<0$ hence the complex index of refraction is purely imaginary. However, in the region of resonance, the permittivity and permeability may both be positive or negative and so the real part of $n$ is valid and follows the signs of $\mu_{r}$ and $\epsilon_{r}$.

\section{Real part of extracted permeability from a $2 \times 2$ CLS array unit cell}

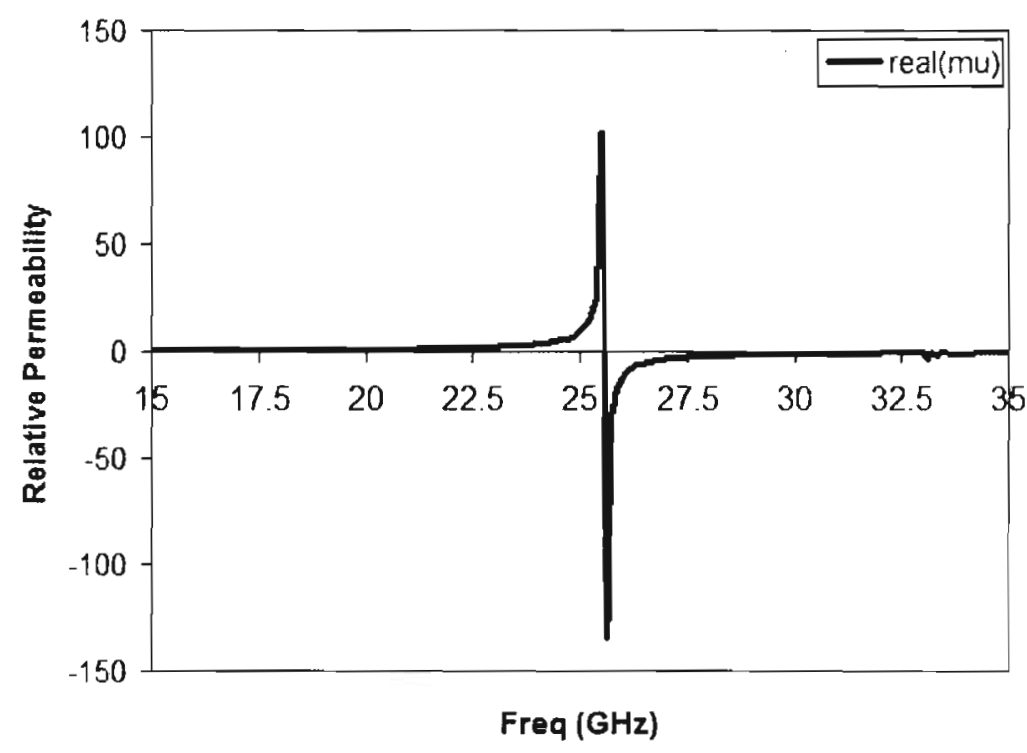

Figure 6.12 Extracted $\mu_{r}$ from S-parameters. The equation (2.28) is used for extraction. 


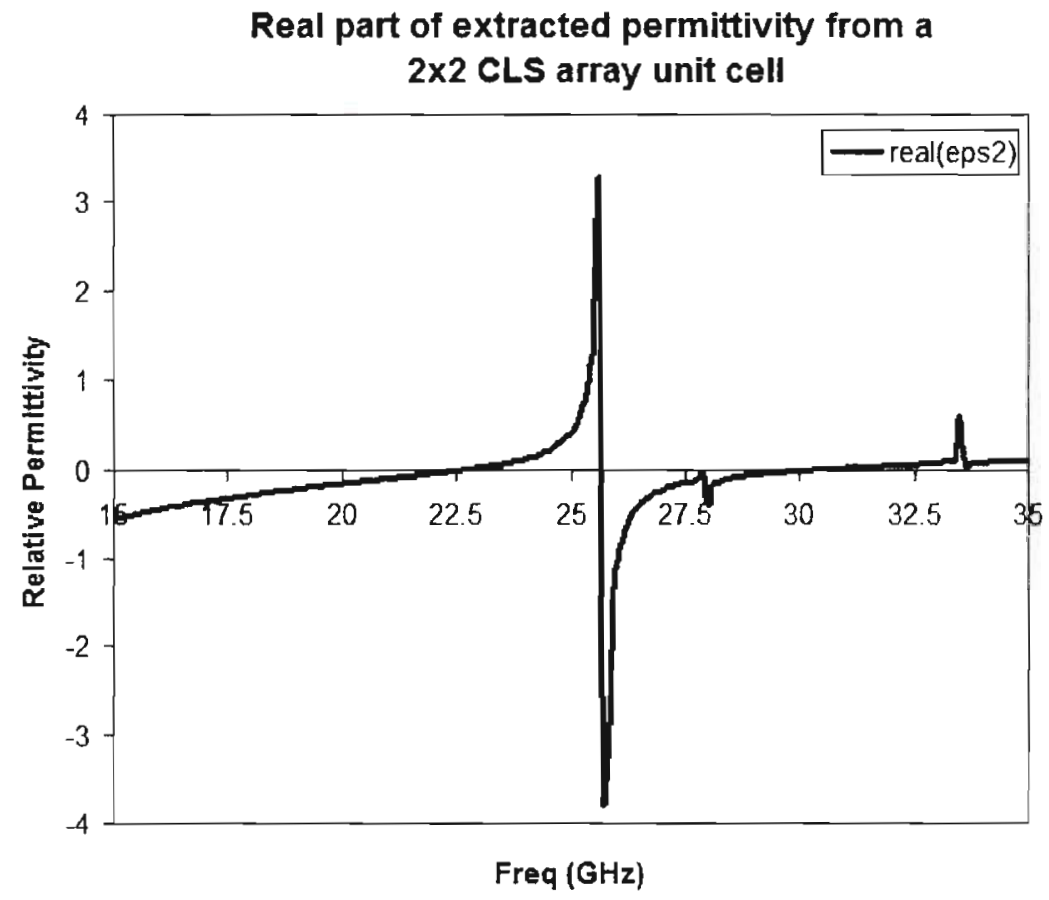

Figure 6.13 Extracted $\epsilon_{r}$ from $S$-parameters recorded through the simulation of a $2 \times 2$ CLS array.

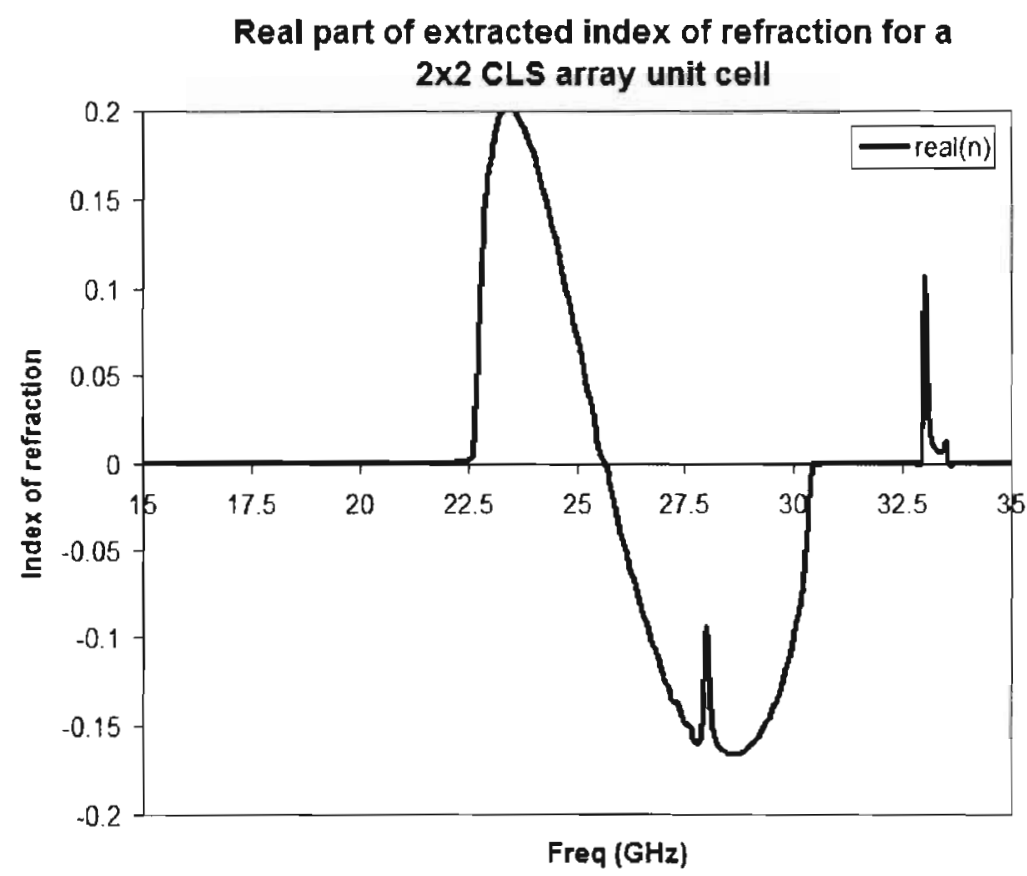

Figure 6.14 Extracted index of refraction from $S$-parameters recorded through simulation of a $2 \times 2$ CLS array. 


\section{Chapter 7}

\section{Copper inclusion parametric analysis and optimization}

\subsection{Objective}

The dimensions for the split ring resonator are important in determining the frequency response and resonant frequency of the microstrip structure. Longer strip lengths have higher inductance and hence reduce the resonant frequency based on the basic formula $w_{0}=\frac{1}{\sqrt{L C}}$. Other parameters that strongly effect experimental data is the dielectric permittivity $\epsilon_{r}$ and unit cell thickness. Ansoft HFSS is used to vary parameters of the split ring unit cell and record the forward transmission $\left(S_{21} \mathrm{~dB}\right)$ and return loss $\left(S_{11} \mathrm{~dB}\right)$. With apriori knowledge of the dielectric permittivity and perhaps the substrate and unit cell thickness (constrained due to ordered parts specs), the unit cell diameter, trace widths and/or gap widths may be varied. The variables which produce a positive inflection in $S_{21}$ (minima) and negative inflection in $S_{11}$ (maxima) are considered as prospective parameters for negative permeability at the frequency of interest. Note that the characteristics of a material with negative permeability only are opposite to those frequency characteristics of a double negative metamaterial. 


\subsection{SRR Parameters}

The diagram below depicts a standard square SRR unit cell.

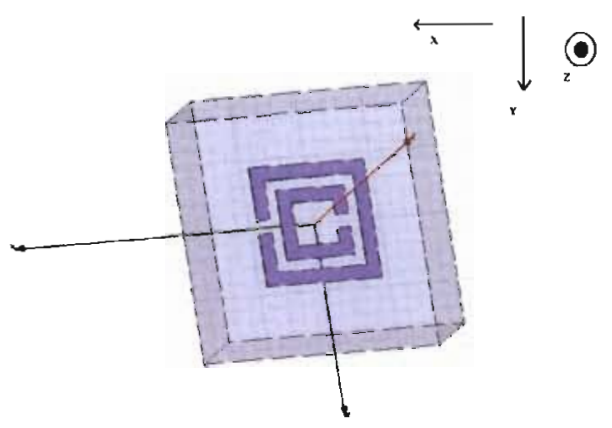

Figure 7.1 Standard square SRR unit cell.

A description of the parameters is given in the table below:

\begin{tabular}{|c|c|}
\hline Parameter & Description \\
\hline \hline a & Split ring resonator diameter (tangential distance from center to outer edge) \\
\hline $\mathrm{c}$ & Microstrip Width \\
\hline $\mathrm{d}$ & Gap width between Outer and inner ring \\
\hline $\mathrm{t}$ & Thickness of unit cell (dielectric substrate + spacers. \\
\hline copper & Height of traces $-\frac{1}{2} \mathrm{Oz}$. Copper has height of 0.708 mils \\
\hline
\end{tabular}

Table 7.1 Square SRR parameters

\subsection{Parameteric sweep results}

\subsubsection{Square/Quadrilateral SRR - Diameter sweep}

In this parametric analysis, the diameter of the Square split ring resonator is varied and the values for $\left|S_{11}\right| \mathrm{dB}$ and $|S 21| \mathrm{dB}$ are recorded. Only the variable $a$ is altered. Below are initial sweeps for a single square SRR on FR4 substrate. The target frequency is set to $8.5 \mathrm{GHz}$.

The frequency response for the SRR resonators on FR4 show two potential areas where negative 


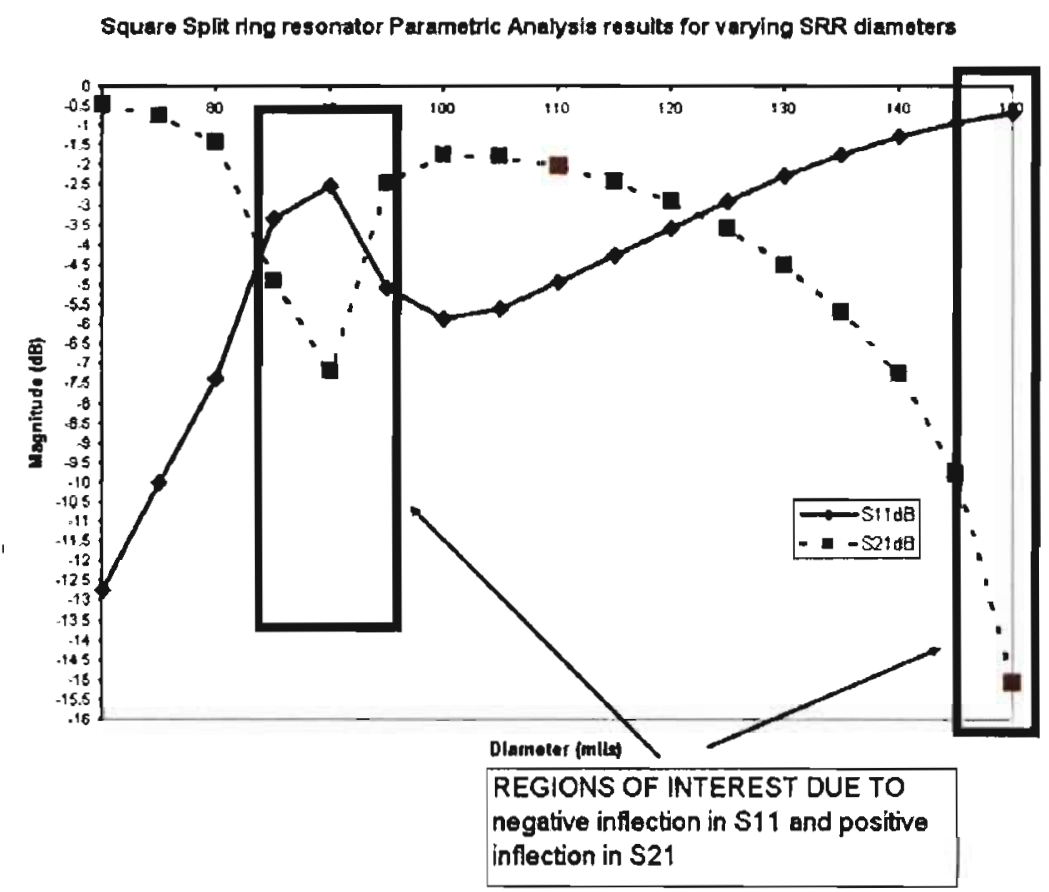

Figure 7.2 Parametric Analysis results for square SRR on FR4 substrate with 'a' varied from 70 to 150 mils in 5 mil steps. ' $c^{\prime}=10$ mils 'd' $=8$ mils and $t=62$ mils.

inflection in $S_{11}$ and positive inflection in $S_{21}$ occur, hence resonance. In order to be sure that the two respective values of 'a' produce negative permeability, the two values are individually simulated in HFSS.

Figure (7.3) clearly shows a resonance in both forward transmission and insertion loss. On the otherhand, Figure (7.4) clearly shows that around the target frequency of $8.5 \mathrm{GHz}$, there is no negative inflection in $S_{11}$ and positive inflection in $S_{21}$. Due to this, parametric analysis for the SRR on FR-4 substrate will occur in the region of a $=90$ mils. Further parametric analysis occurs in the region for $\mathrm{a}=85$ mils to $\mathrm{a}=95$ mils.

Figure (7.5) clearly shows that resonance occurs at $8.5 \mathrm{Ghz}$ when $\mathrm{a}=88 \mathrm{mils}$. The previous value for $a=90$ mils showed a frequency shift in resonance. 


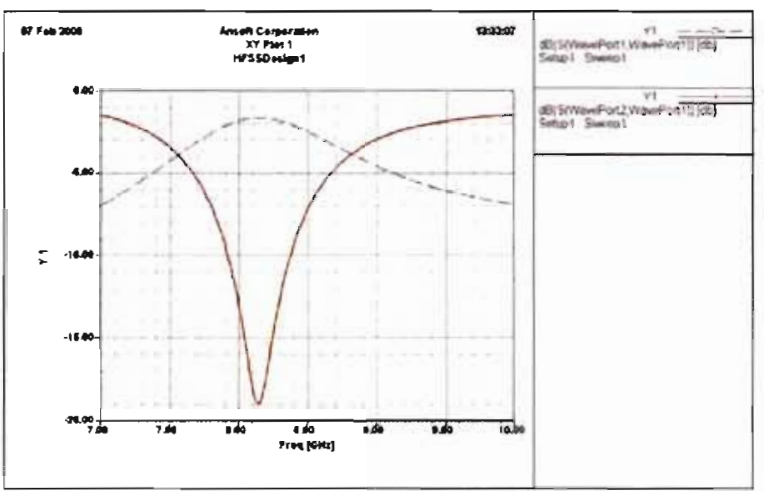

Figure 7.3 Insertion loss and forward transmission for an SRR on FR4 unit cell with $\mathrm{a}=$ 90 mils.

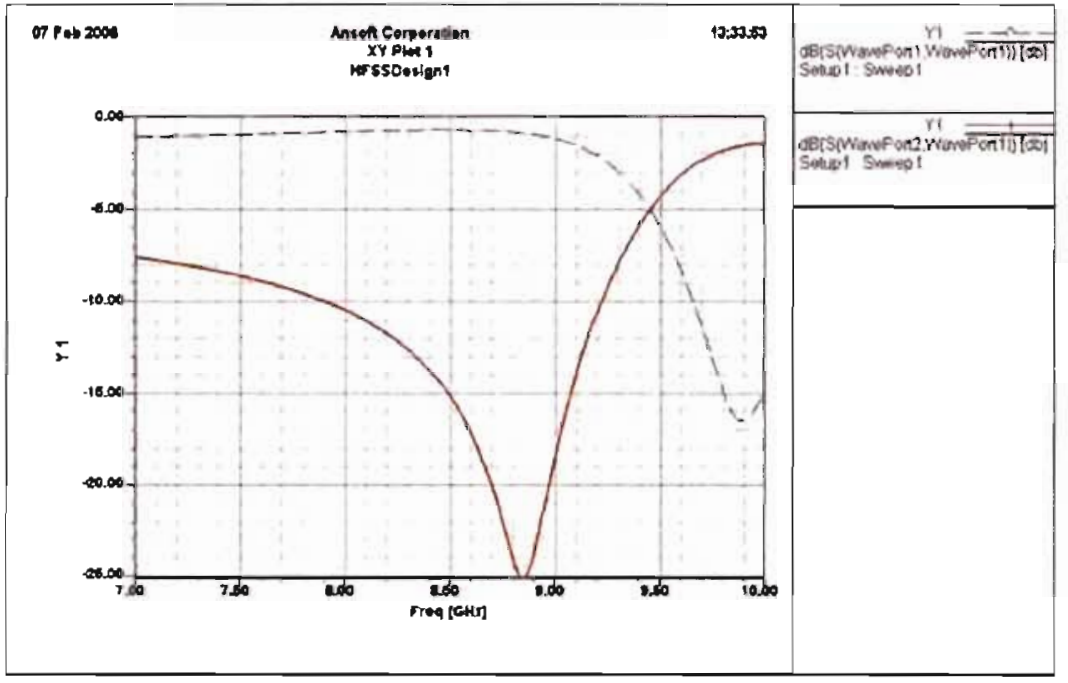

Figure 7.4 Insertion loss and forward transmission for an SRR on FR4 unit cell with $\mathrm{a}=$ 150 mils.

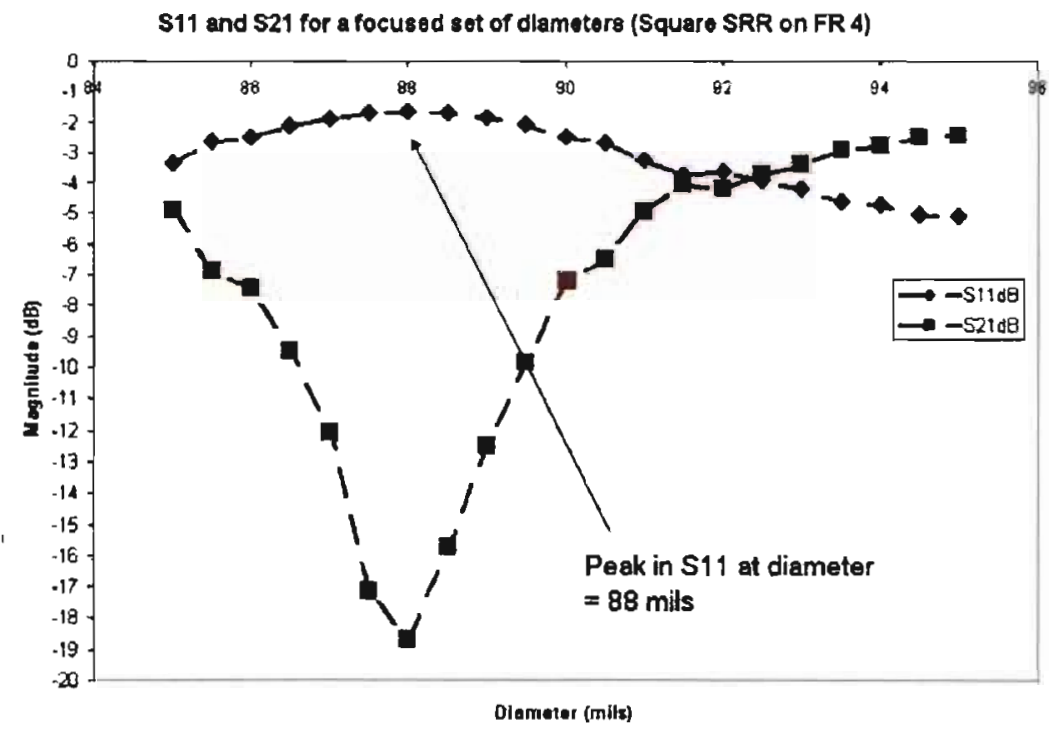

Figure 7.5 Parametric Analysis results for square SRR on FR4 substrate with 'a' varied from 85 to 95 mils in 0.5 mil steps. ' $c$ ' $=10$ mils 'd' $=8$ mils and $t=62$ mils. 


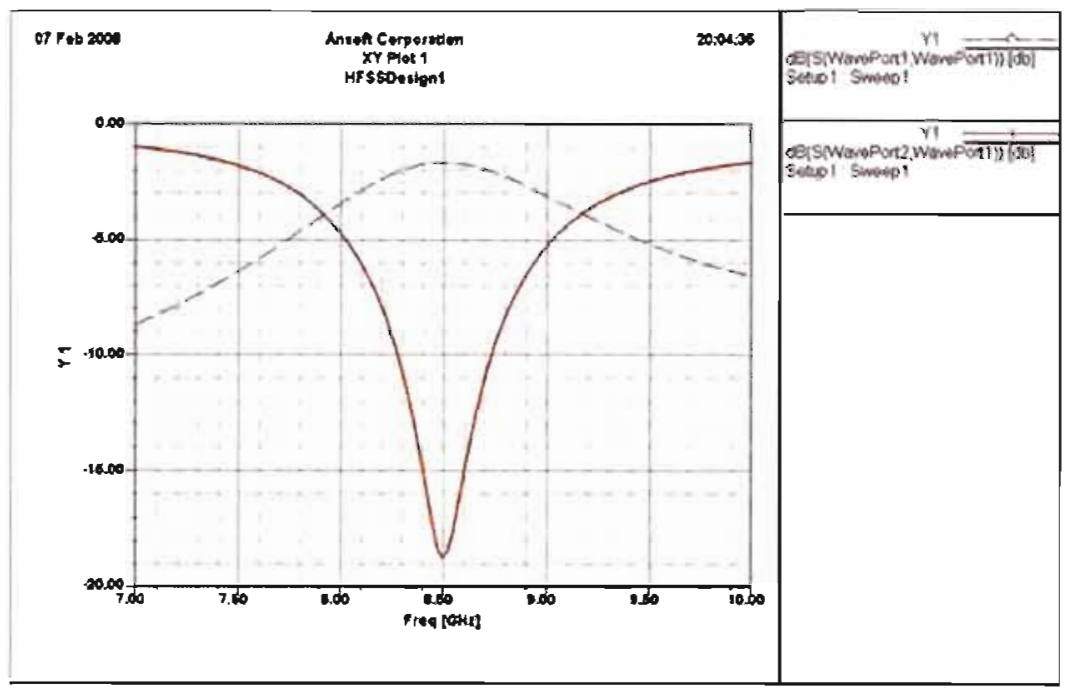

Figure 7.6 Insertion loss and forward transmission for an SRR on FR4 unit cell with a = 88 mils.

\subsection{Planar versus non-planar designs}

A non-planar metamaterial has a non-zero vertical spacing between the split ring resonator structures and the capacitive loaded strips. Several simulations in HFSS are performed to determine the transmission characteristics for varied vertical spacing between the two types of inclusions. The vertical spacing is varied in accordance with common substrate thicknesses: 16, 32, 64 mils. The parameters recorded and compared to find the ideal vertical spacing for fabrication are: $|S 21| \mathrm{dB}$, $|S 11| \mathrm{dB},|S 21|,|S 11|$ and resonant frequency $f_{0}$. A table outlining the results of the simulations is provided below:

\begin{tabular}{||c|c|c|c|c||}
\hline & $\begin{array}{c}0 \text { mil spacing } \\
\text { (planar) }\end{array}$ & $\begin{array}{c}\text { 16mil spacing } \\
\text { (non-planar) }\end{array}$ & $\begin{array}{c}\text { 32mil spacing } \\
\text { (non-planar) }\end{array}$ & $\begin{array}{c}64 \text { mil spacing } \\
\text { (non-planar) }\end{array}$ \\
\hline$|S 11| \mathrm{dB}$ & $-9.08 \mathrm{~dB}$ & $-7.06 \mathrm{~dB}$ & $-3.97 \mathrm{~dB}$ & $-2.5 \mathrm{~dB}$ \\
\hline$|S 21.| \mathrm{dB}$ & $-10.37 \mathrm{~dB}$ & $-13.06 \mathrm{~dB}$ & $-19.35 \mathrm{~dB}$ & $-43.4 \mathrm{~dB}$ \\
\hline$|S 11|$ & 0.35 & 0.44 & 0.64 & 0.75 \\
\hline$|S 21|$ & 0.3 & 0.22 & 0.11 & $\approx 0$ \\
\hline$f_{0}$ & $8.6 \mathrm{GHz}$ & $8.5 \mathrm{GHz}$ & $8.35 \mathrm{GHz}$ & $8.0 \mathrm{GHz}$ \\
\hline
\end{tabular}

Table 7.2 Comparison of various transmission test results for a SRR-CLS unit cell where vertical electrical spacing between the CLS and SRR inclusions is varied. Measurements taken at the resonance frequency $f_{0}$

The results clearly show that at the resonance frequency a planar structure with no vertical 
spacing between the SRR and CLS structures provides increased transmission in the passband. As vertical spacing increases, not only does the resonance frequency shift (hundreds of $\mathrm{MHz}$ ), but $|S 21|$ decreases. At larger spacings such as $64 \mathrm{mil}$, no signal is transmitted through the metamaterial. The FR-4 as explained previously is a lossy material, so none of the designs show complete matching to freespace. However, the planar design clearly has higher transmission in boards with vertical spacings of 16 mil boards by $3 \mathrm{~dB}$ for $|S 21| \mathrm{dB}$ and has a larger attenuation in $|S 11| \mathrm{dB}$ at resonance by $2 \mathrm{~dB}$.

\subsection{S-SRR Parameters}

In this parametric analysis the diameter of the S-Split ring resonator is stepped and the values for $S_{11} \mathrm{~dB}$ and $S 21 \mathrm{~dB}$ are recorded. Only the variable $a$ is varied. Below are initial sweeps for a single Square SRR on FR4 substrate. The target frequency is set at $8.5 \mathrm{GHz}$.

Diagram 7.7 depicts a standard square S-SRR unit cell.

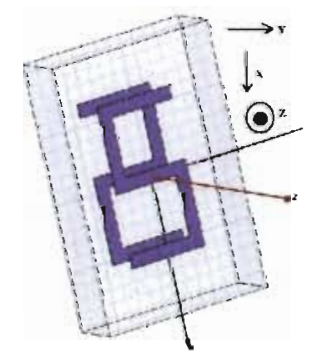

Figure 7.7 Standard S shaped SRR unit cell.

A description of the parameters is given in Table 7.3.

Figure (7.8) clearly shows that the $\mathrm{S} 21$ peak and $\mathrm{S} 11$ trough occur at $\mathrm{w}=6.3 \mathrm{~mm}$. Assuming the change in the length alters the inductance and therefore the resonant frequency, the inflection in both parameters occurs at $\mathrm{w}=6.3 \mathrm{~mm}$ for a target frequency of $8.5 \mathrm{Ghz}$. To confirm this, a frequency sweep with the parameters in question is performed to confirm resonance at $8.5 \mathrm{GHz}$. 


\begin{tabular}{|c|c|}
\hline Parameter & Description \\
\hline \hline $\mathrm{w}$ & S-SRR length (X direction above) \\
\hline $\mathrm{h}$ & S-SRR width (Y direction) \\
\hline $\mathrm{a}$ & total length of unit cell (X direction) \\
\hline b & total width of unit cell (Y direction) \\
\hline c & Microstrip Width \\
\hline $\mathrm{d}$ & Gap size between mirrored S-Shaped inclusions. \\
\hline l & Thickness of unit cell (dielectric substrate + spacers. \\
\hline copper & Height of traces $-\frac{1}{2}$ Oz. Copper has height of 0.708 mils \\
\hline
\end{tabular}

Table 7.3 S-SRR parameters

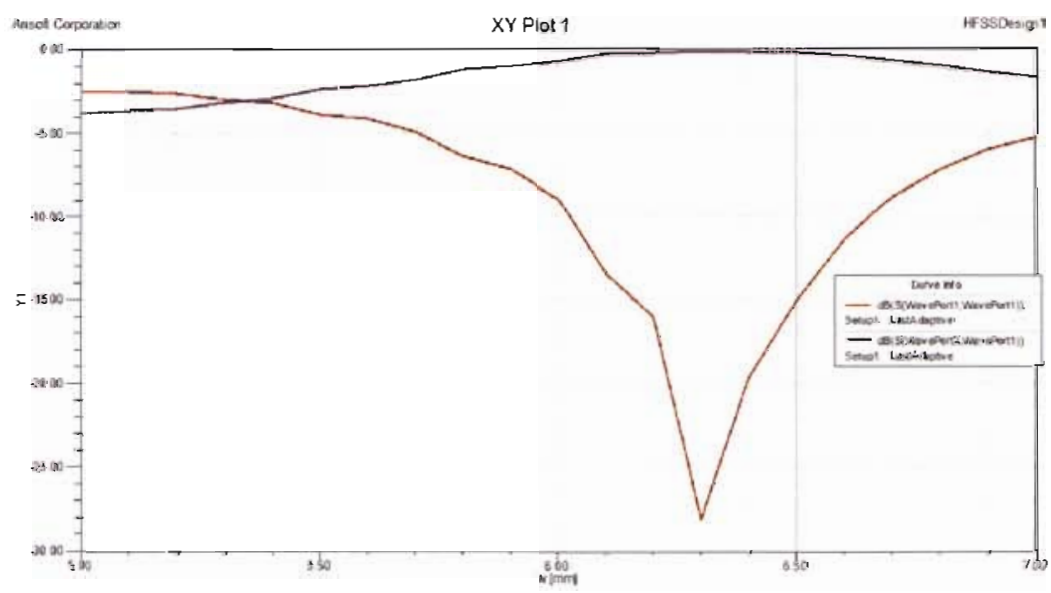

Figure 7.8 Parametric Analysis results for S-shaped SRR on FR4 substrate with 'w' varied from 5 to $7 \mathrm{~mm}$ in $0.1 \mathrm{~mm}$ steps. Constants: ' $h{ }^{\prime}=3 \mathrm{~mm}$ ' $c$ ' $=0.5 \mathrm{~mm}$ ' $a{ }^{\prime}=10 \mathrm{~mm}$ 'b' $=6.5 \mathrm{~mm}$ 'd' $=1.57 \mathrm{~mm}$, 'l' $=2.362 \mathrm{~mm}$. 'd' $=8$ mils and $\mathrm{t}=62$ mils. 


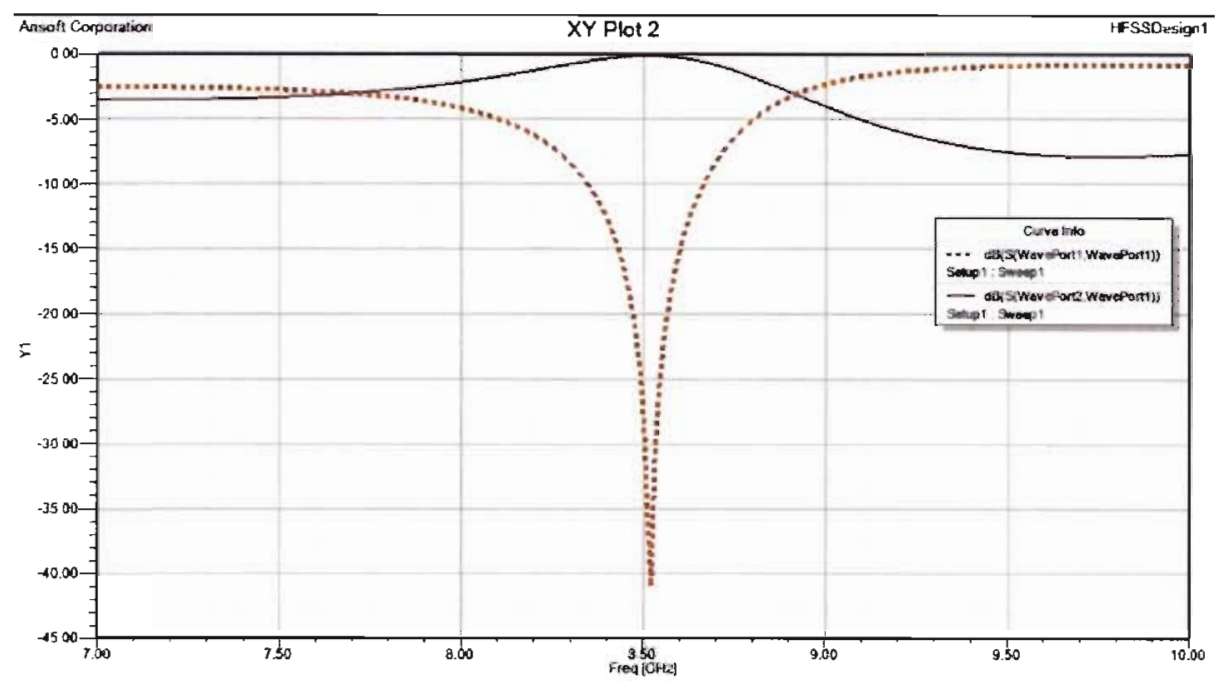

Figure 7.9 Measured S-paramters show resonance at $8.5 \mathrm{GHz}$ when ' $w$ ' $=6.3 \mathrm{~mm}$

As is seen in Figure 7.9, resonance does infact occur at approximately $8.5 \mathrm{GHz}$ and one can move onto design of the final S-SRR metamaterial.

\subsection{Final Metamaterial transmission simulations}

Firstly, the square SRR structure was grouped with capacitively loaded strips below to form a planar structure. There is no vertical spacing between the capacitively loaded strips and the split ring resonators. 


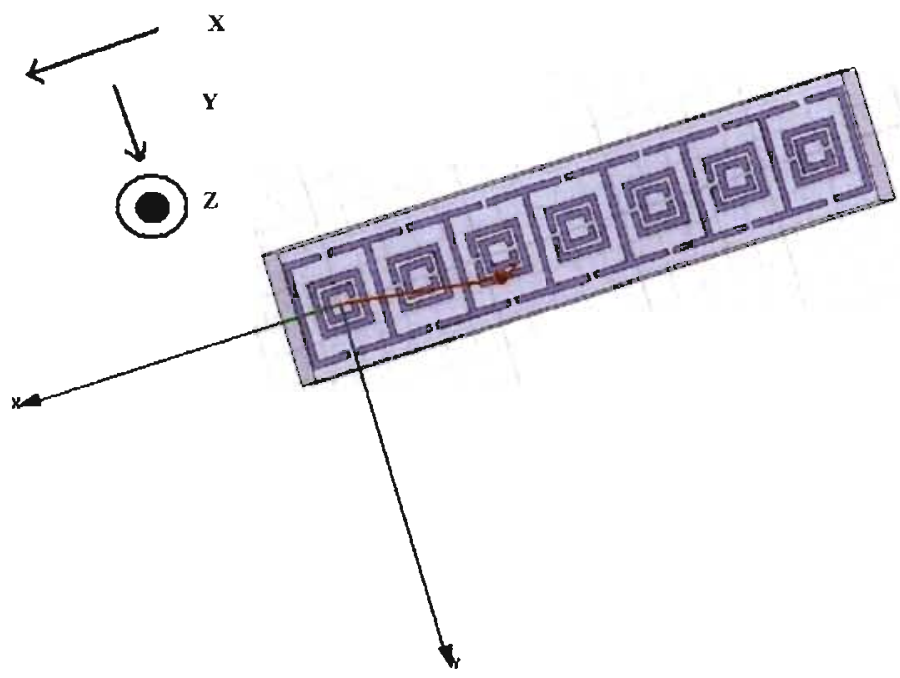

Figure 7.10 Final unit cell structure for the planar SRR-CLS metamaterial. The value of 'a' found through parametrics is used.

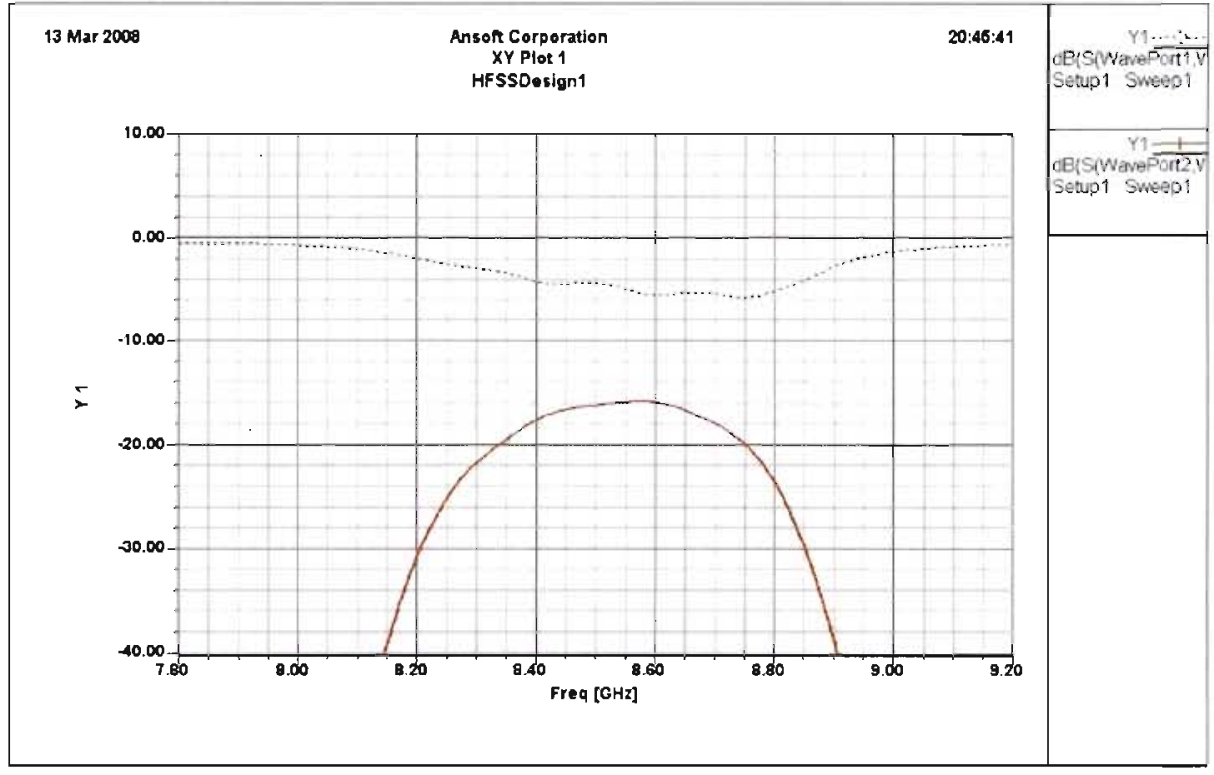

Figure 7.11 Transmission simulation results for the SRR-CLS structure. Resonance is visible at $8.5 \mathrm{GHz}$. 
The S-shaped split ring resonator structure when placed into a unit cell with other s-shaped split ring resonators also showed the desired resonance at $8.5 \mathrm{GHz}$.

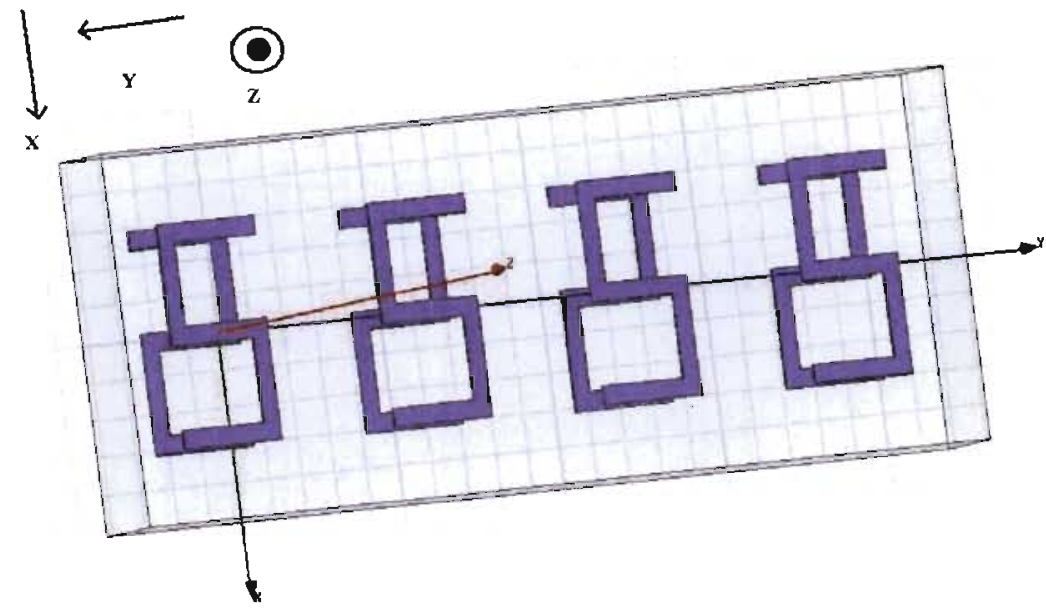

Figure 7.12 Final unit cell structure for the planar S-SRR metamaterial.

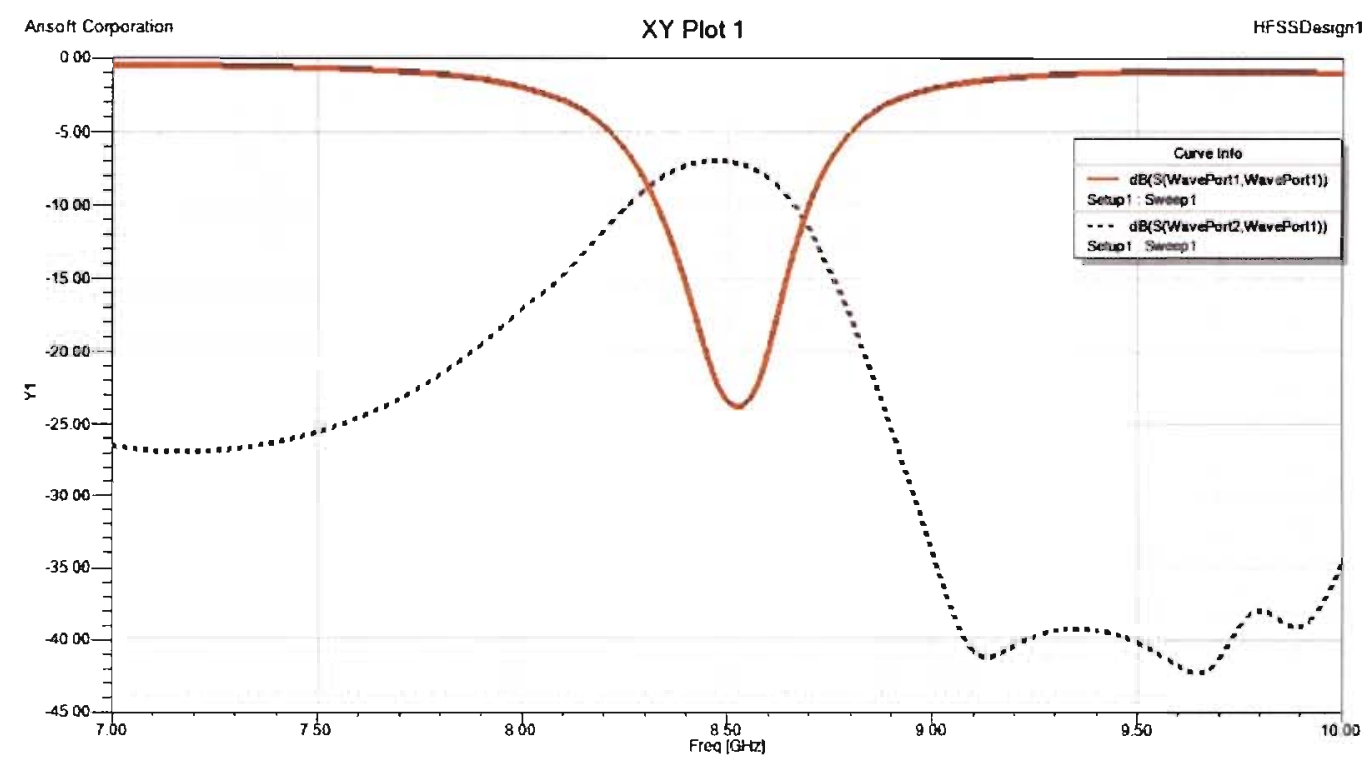

Figure 7.13 Transmission simulation results for the S-SRR structure. Resonance is visible at $8.5 \mathrm{GHz}$. 


\section{Chapter 8}

\section{Metamaterial prism simulation in HFSS}

To investigate the refractive properties of metamaterials and capability of the designed copper inclusions in a periodic structure to act as a negative refractive index metamaterial, simulations are conducted in Ansoft HFSS prior to metamaterial design finalization and structure fabrication. First, the simulation setup is introduced followed by testing of an ideal bulk material whose permeability and permittivity are set directly through the software to simulate perfectly homogeneous materials. These values are varied and the peak angle of power transmission recorded to obtain the prism's index of refraction. These values are compared to theoretical values. Finally, a prism with S-shaped SRR (S-SRR) inclusions finalized for resonance at $8.5 \mathrm{GHz}$ is simulated. Far-field radiation results are obtained at several frequencies to view the frequency dependent properties of this metamaterial.

\subsection{Simulation setup}

A top down view of the test setup is shown below. A waveport with one dominant mode defined by the integration line is used as the input. To reduce simulation time and reduce the simulation space, a free space setup is not used here. Instead a parallel plate setup is created with perfect E planes (X-Y plane). A lossy material (emulating absorbent foam) is used to constrain the EM energy and a TEM mode wave is incident on the prism front face. This lossy material may be defined in HFSS as a material with dielectic loss tangent $\delta>>1$. Like the transmission slab simulations, 
the prism is covered by perfect-E and perfect $\mathrm{H}$-planes to force the EM wave inside the structure to resemble a plane wave. As one is concerned with azimuthal refraction the setup is not large in the $\mathrm{Z}$ direction ( a mere $10 \mathrm{~mm}$ compared to the $\mathrm{X}$ and $\mathrm{Y}$ directions). HFSS uses periodic boundary conditions to yield accurate results in the $\mathrm{YZ}$ plane (H-plane). Radiation boundaries line the sides of the test setup. These radiation boundaries minimize reflections off the side walls. They are simpler to implement than phase matched layers and allow radiation to reach the far zone. When radiation boundaries are assigned, one can create far-field radiation patterns by defining an infinite sphere in HFSS. Phi and theta scans may be defined. In the case of refraction testing, one is interested in angles $\phi=\left\{0^{\circ}, 1^{\circ}, \ldots 180^{\circ}\right\}$ and $\theta=90^{\circ}$.

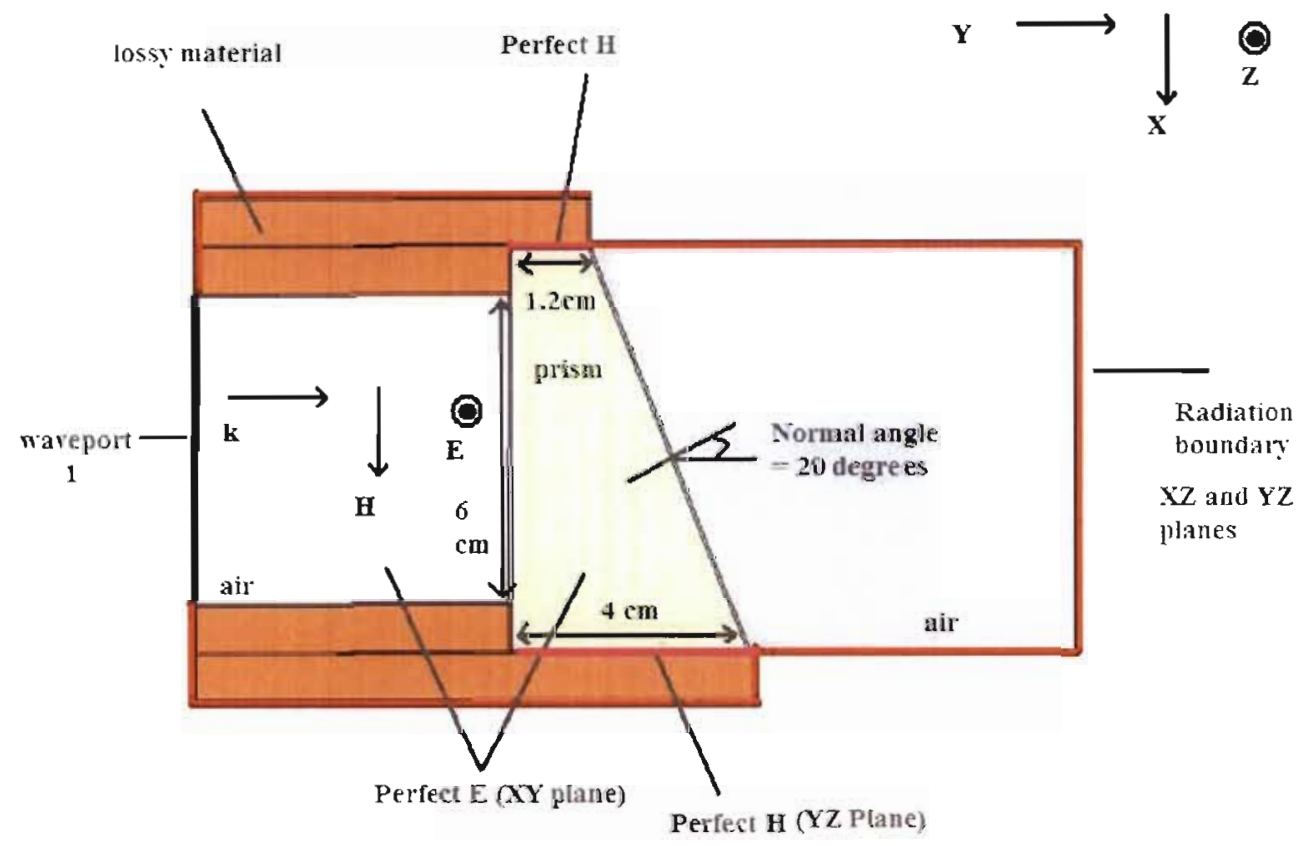

Figure 8.1 HFSS simulation setup for prism refraction setup. A waveport is used as an input. Radiation boundaries allow waves to radiate into the far-field. Top-down view does not show depth in Z-direction $(10 \mathrm{~mm})$.

\subsection{Ideal simulations}

Simulations are carried out with no defined inclusions within the prism. The prism dielectric loss tangent is negligible. The bulk parameters $\epsilon_{r}$ and $\mu_{r}$ are varied directly in HFSS while keeping the 
prism angle defined by its normal angle constant in size. The normal angle may be calculated using the simple equation:

$$
\text { normal angle }=\tan ^{-1}\left(\frac{L_{\text {side } 2}-L_{\text {side } 1}}{L_{\text {incident } f a c e}}\right)
$$

The peak power transmission angle is found with respect to the normal angle. Hence the normal angle $\phi_{\text {normal }}$ is normalized to zero degrees.

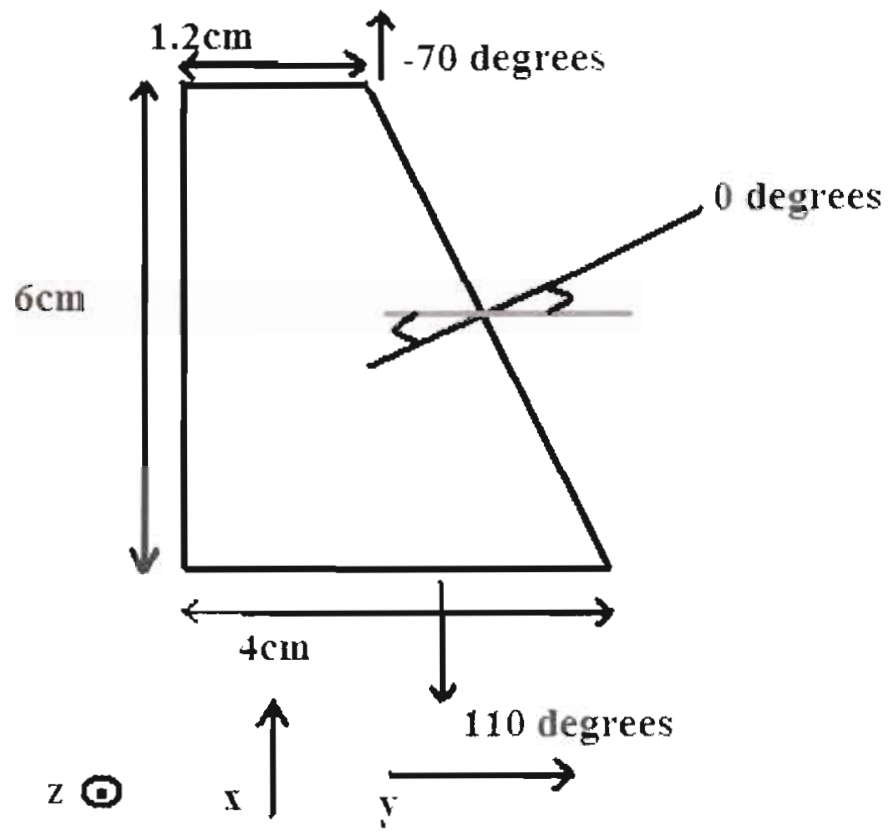

Figure 8.2 Prism outline indicating angles with respect to 0 degrees normalized to the prism normal face. 


\begin{tabular}{||c|c|c|c|c|c||}
\hline$\epsilon_{r}$ & $\mu_{r}$ & Peak transmission angle (deg) & Theoretical $n$ & Simulated $n$ & \%difference \\
\hline 2.2 & 1 & 26.04 & 1.48 & 1.37 & 8 \\
\hline 1 & 1 & 16.04 & 1 & 0.85 & 15 \\
\hline 0.5 & 1 & 8.04 & 0.31 & 0.11 & 65 \\
\hline-0.5 & -1 & 1.04 & -0.7 & 0.002 & 98 \\
\hline-1 & -1 & -22.96 & -1 & -1.09 & 9 \\
\hline-2.2 & -1 & -27.96 & -1.48 & -1.41 & 4.7 \\
\hline-2.9 & -1 & -40.96 & -1.70 & -1.75 & 2.9 \\
\hline
\end{tabular}

Table 8.1 Table showing agreement between theoretical calculations for $n$ using $n=$ $\sqrt{\epsilon_{r} \mu_{r}}$ and simulated results. Agreement is accurate in regions where $|n| \geq 1$.

Figure 8.4 shows that a left hand material with negative $\epsilon$ and $\mu$ exhibits refraction to the negative side of the prism normal. However, one cannot cpntinue to decrease $\epsilon$ and $\mu$. A large positive or negative index of refraction may lead to total internal reflection within the prism. If $\left|n_{1}\right| \sin \theta_{1}>1$, the calculation for $\theta_{2}$ is undefined. Hence $\theta_{2}=\sin ^{-1}\left(\frac{n_{1} \sin \theta_{1}}{n_{2}}\right)$ is undefined.

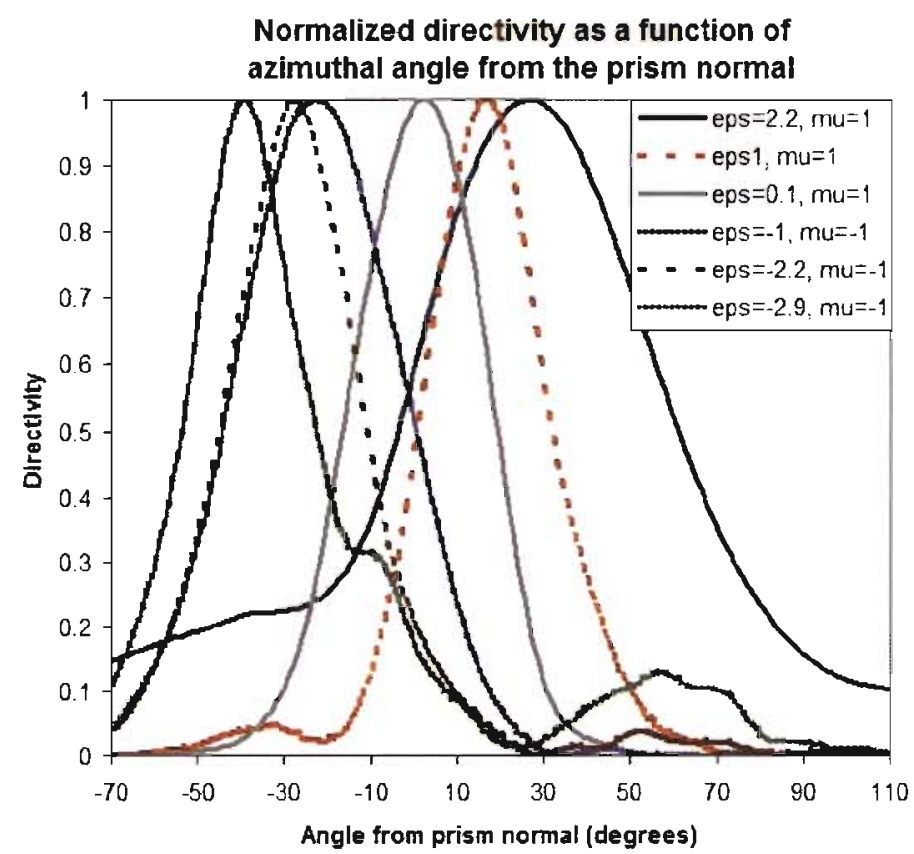

Figure 8.3 Normalized directivity simulations for ideal prism sample for varying $\epsilon_{r}$ and $\mu_{r}$. 


\subsection{Simulation with metal inclusions}

In this section, the S-shaped SRR inclusions parametrically optimized in chapter 9 are embedded into the prism medium. The prism dielectric material is FR4 $\left(\epsilon_{r}=4.4, \mu_{r}=1\right)$ and the $S$ shaped inclusions have been designed to have a resonance in permeability at $8.5 \mathrm{GHz}$. Other than placing the inclusions in a staircase pattern as shown in figure 9.3 , no other aspects of the simulation setup are changed. Hence, angles of refraction are calculated as in the previous section. Note that frequency sweeps save time for smaller designs. However, the large number of inclusions takes its toll on the solver software and the HFSS software may abort when it runs short on memory. The PC used to run simulations is a Quad-core, 4GB memory machine. However, a limitation seen during simulations is that the software is prone to memory leakage due to poor software development. Hence memory shortage errors occur more often for large designs. In these cases, single frequency simulations may be more beneficial. Furthermore, only one layer in the $z$ direction is simulated to save time. Periodic boundary symmetry conditions in HFSS allow one to take a cut out of a plane such as a prism and extrapolate the results for a much larger structure.

Table 8.2 identifies the peak angle of refraction from the prism normal at a given simulation frequency and the extracted index of refraction using Snell's equation.

\begin{tabular}{|c|c|c||}
\hline EM radiation frequency $(\mathrm{GHz})$ & refraction angle $(\mathrm{deg})$ & Index of refraction \\
\hline 7 & 84 & 2.91 \\
\hline 7.5 & 10 & 0.51 \\
\hline 7.8 & -52 & -2.35 \\
\hline 8.2 & -60 & -2.53 \\
\hline 8.5 & -50 & -2.23 \\
\hline 9.0 & -20 & -0.90 \\
\hline 9.5 & 26 & 1.28 \\
\hline
\end{tabular}

Table 8.2 Table showing frequency dependent index of refraction obtained from far field radiation pattern simulations of S-shaped SRR metamaterial prism structure in HFSS.

A prism structure utilizing S-shaped SRR (S-SRR) structures is proposed in chapter 9 with the purpose of fabricating and testing in the Anechoic chamber. The results obtained here provide a good comparison tool. 


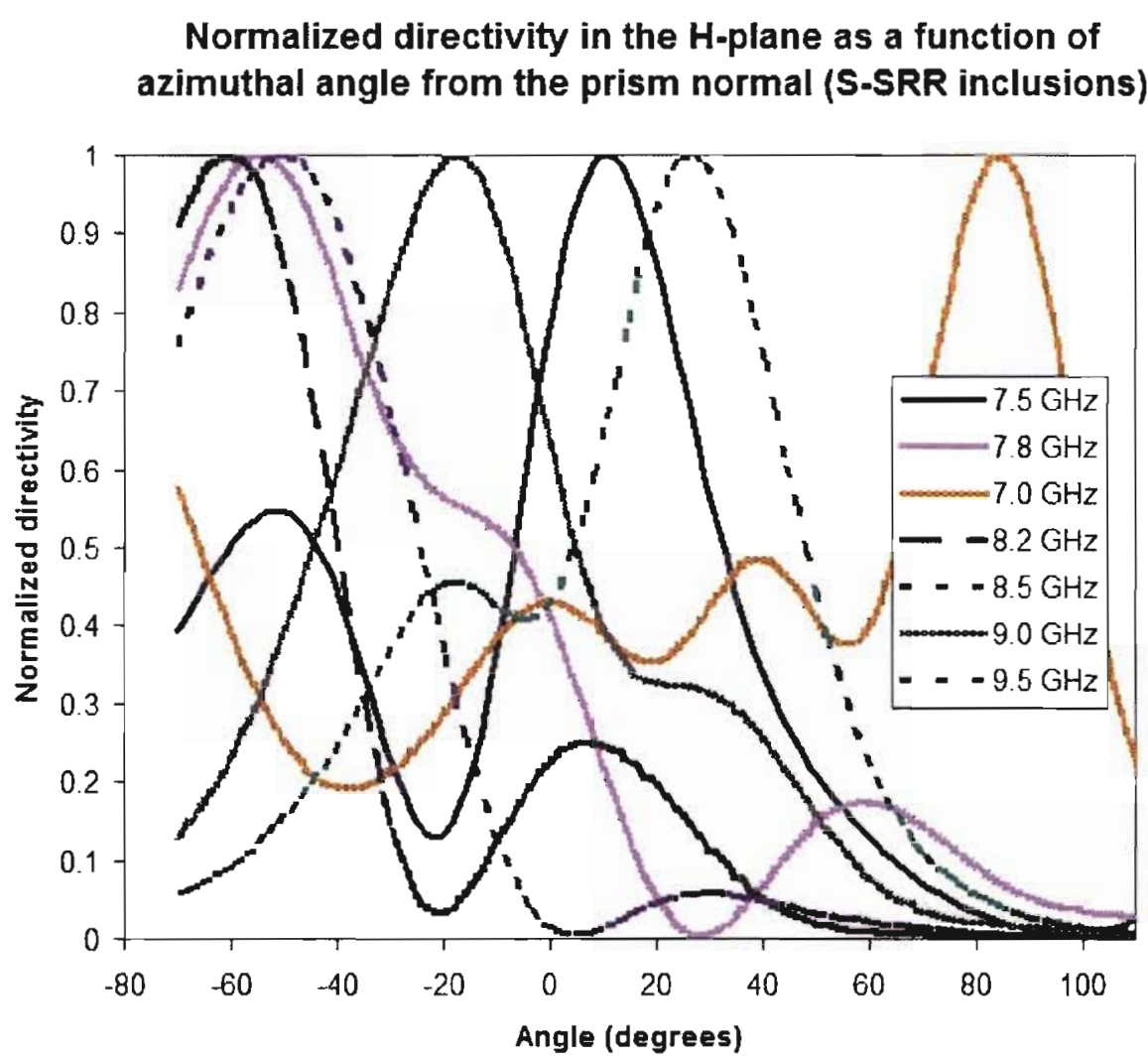

Figure 8.4 Normalized directivity with respect to the peak recorded value for a given frequency. Peak directivity is visible at angles negative of the prism normal for frequencies such as $7.8 \mathrm{GHz}, 8.2 \mathrm{GHz}, 8.5 \mathrm{GHz}$ and $9 \mathrm{GHz}$. The prism is operating as a normal right-hand material at $7 \mathrm{GHz}$ and $9.5 \mathrm{GHz}$. 


\section{Chapter 9}

\section{Final metamaterial structure designs}

This chapter serves to introduce the dimensions of the metamaterials to be fabricated and tested in the Anechoic Chamber. Microstrip boards for three separate designs are fabricated by an external supplier. The first design makes use of the common split ring resonator (SRR) and capacitively loaded strip (CLS) structures. The second design utilizes a newer inclusion named the S-Shaped split ring resonator. The performance of this structure shall be compared to that utilizing a standard SRR-CLS design. Finally, a prism structure is fabricated to view the refractive phenomenon in Double negative metamaterials.

\subsection{SRR/CLS structure}

A diagram depicting the outline and dimensions of the 3D structure is shown in figure 9.1.

The copper inclusions will be placed in the $X-Z$ plane. All copper traces shall be etched on 62mil FR-4 board. A 31 mil spacer FR-4 board shall be included. Hence, each unit cell has a depth of 93 mils in the $\mathrm{Y}$ direction. The number of required layers can be found by converting $93 \mathrm{mils}$ to $\mathrm{cm}(0.236 \mathrm{~cm})$ to find $l_{\text {cell }}$ and calculating the ratio:

$$
\frac{l_{y}}{l_{c e l l}}=\frac{13 \mathrm{~cm}}{0.236 \frac{\mathrm{cm}}{c m_{y}-\text { dir }}} \approx 55 \text { cells in y direction }
$$




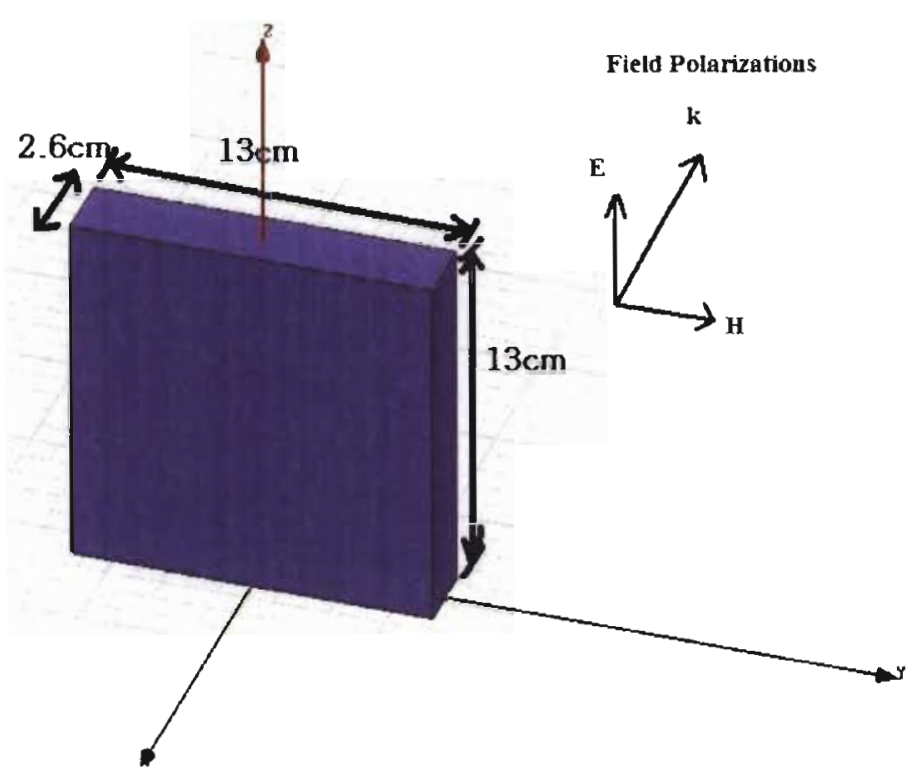

Figure 9.1 Dimensions for Metamaterial structure containing SRR/CLS and S-SRR inclusions

Approximately 55 copper etched boards and 55 spacer boards shall be needed for this metamaterial as 1 cell in the y direction is composed of one etched FR-4 board and 1 spacer board. Each FR-4 board has dimensions $(X, Y, Z)$ equal to $(2.6 \mathrm{~cm} \times 13 \mathrm{~cm} \times 13 \mathrm{~cm})$. The large number of boards is necessary to build a large enough structure such that an adequate amount of transmit power from the Narda 641 horn antenna illuminates the metamaterial. Due to the large beam width in the far - field, a larger structure will be partial to incident radiation whereas for a small structure much of it will be wasted. As the metamaterial slab is larger than the horn aperture, much of the main beam where power is highest will completely illuminate the structure. The test plan presented in Chapter 10 looks into depth the required far field distance and estimated beam width for a certain size standard gain horn antenna.

\subsection{S-SRR Structure}

Figure 9.2 depicts an $S$ shaped SRR inclusion (S-SRR).

The overall metamaterial dimensions for this S-SRR structure are identical to those in Figure 9.1. The sole difference is the inclusion shape and size as well as the fact that etching shall occur on 


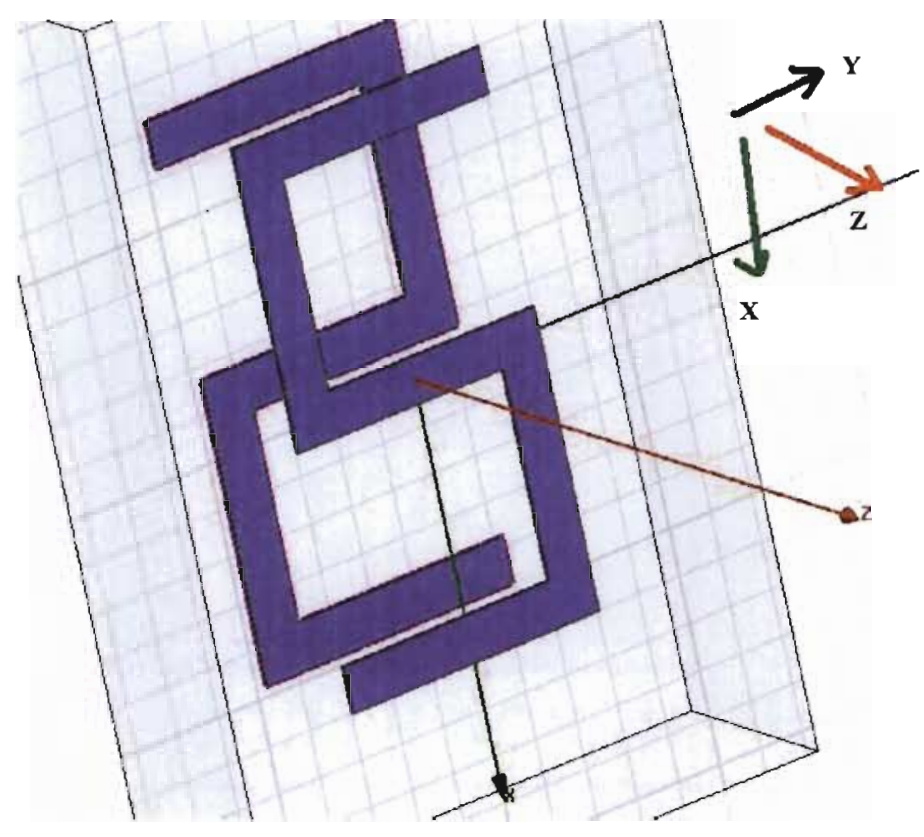

Figure 9.2 Typical S-SRR inclusion

both sides of a 62 mil FR-4 board. The number of etched and fabricated boards is identical to that of the SRR/CLS structure (55 boards each).

\subsection{Prism structure}

A 3D diagram for the outline of the Prism shape is shown below:

A 2D representation of an ideal prism is given in Figure 9.4. The value of $\theta_{p}$ may be calculated as:

$$
\theta_{p}=\tan ^{-1}\left(\frac{6.1 \mathrm{~cm}}{13 \mathrm{~cm}}\right)=25.14^{0}
$$

The angles $\theta_{2 l}$ and $\theta_{2 r}$ are found using Snell's law. The prism's index of refraction is directly 


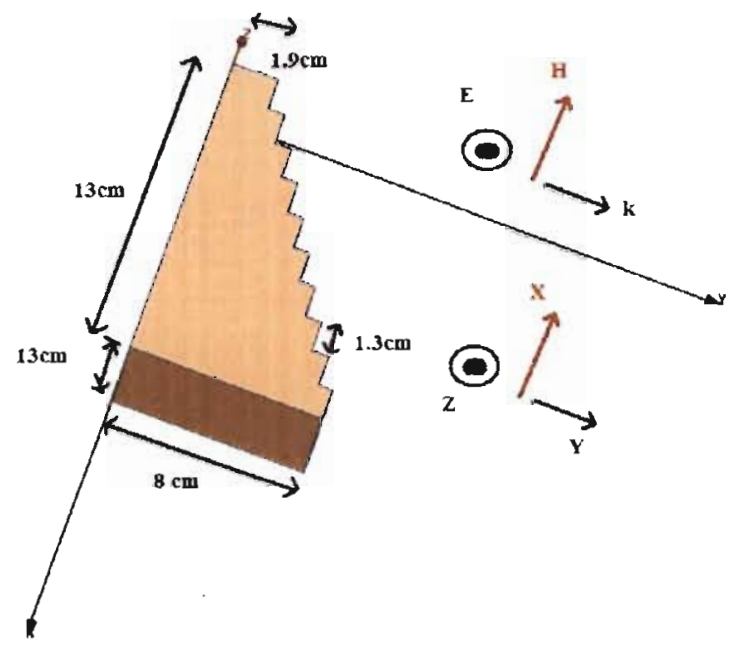

Figure 9.3 Prism S-SRR structure

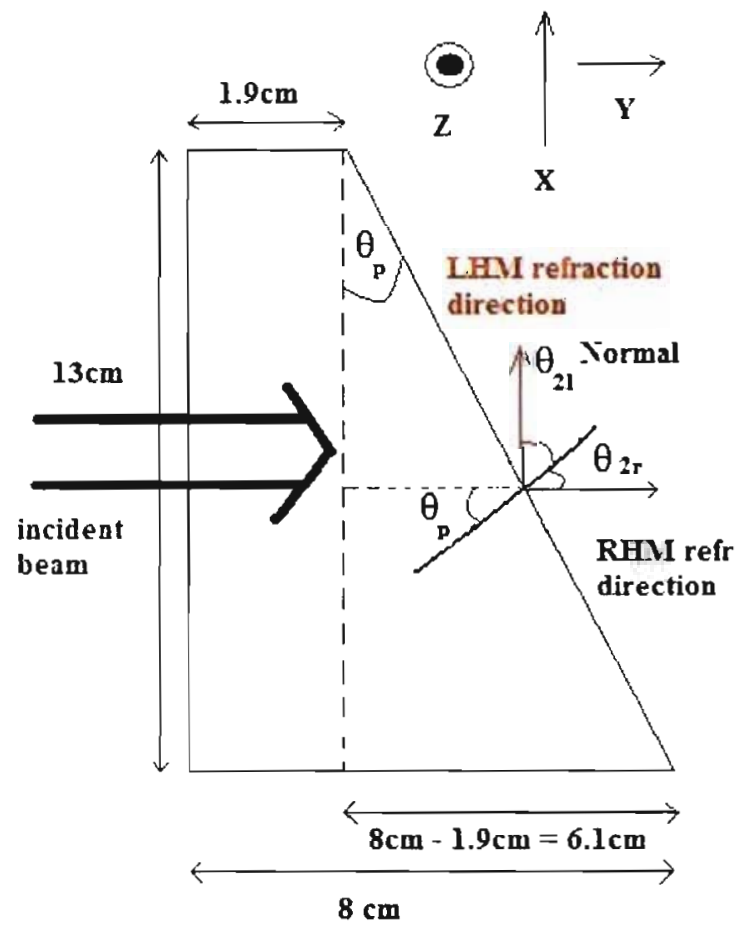

(C) E - field

Figure 9.4 Representation of an ideal prism with the required dimensions 
proportional to refraction angles at the prism-air boundary.

$$
\begin{aligned}
\theta_{2 r} & =\sin ^{-1}\left(\frac{n_{1} \sin \left(\theta_{p}\right)}{n_{2}}\right) \\
\theta_{2 l} & =\sin ^{-1}\left(\frac{-n_{1} \sin \left(\theta_{p}\right)}{n_{2}}\right)
\end{aligned}
$$

The prism contains S-SRR inclusions. Fig 9.3 shows that 10 stacked layers are needed in the prism design. Each individual board design is used in one of the stair prism's steps. Each layer requires a redesign of the unit cell in the $\mathrm{Y}$ direction. For each increasing layer more whole SSRR inclusions were added in the $\mathrm{Y}$ direction while keeping dimensions in the $\mathrm{Z}$ and $\mathrm{X}$ direction constant. A typical design for a layer with 3 S-SRRs in the Y direction is shown below:

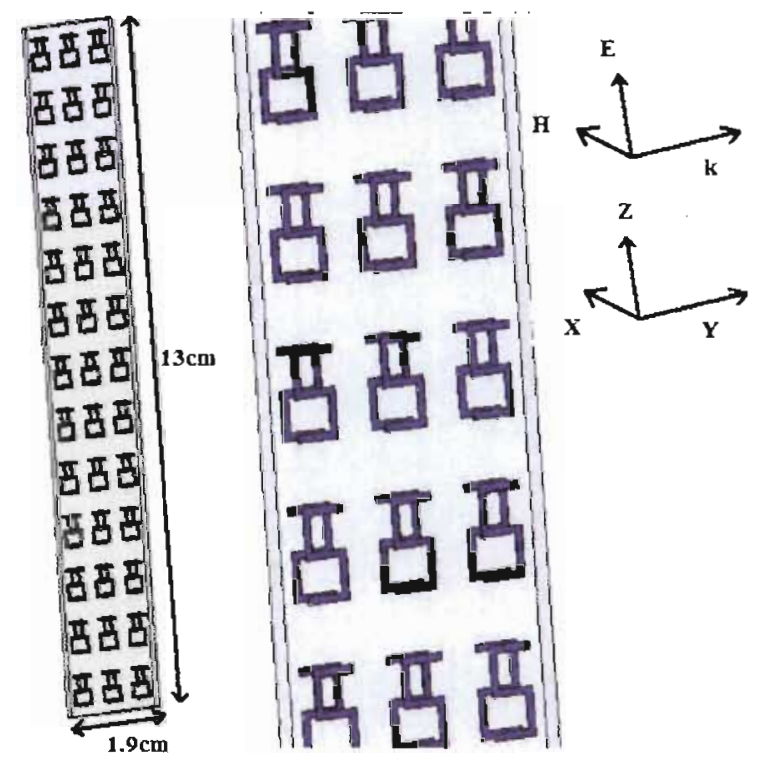

Figure 9.5 Three S-SRRs in Y direction (left to right). Distance totals $1.9 \mathrm{~cm}$.

The design used for the transmission metamaterial slab described initially contains 4 S-SRRs in the $\mathrm{Y}$ direction. This design shall be used along with 9 other designs created in HFSS and converted to Gerber files within ADS. Each design has a different number of unit cells in the direction of wave propagation. A 1 unit cell increase was chosen to minimize the shape and make the structure look more like an ideal prism. The ideal prism would have a perfectly straight edges and flat faces. However, no reasonable method was found to fabricate such a material with common lab equipment. 
Cutting away the inclusions to obtain a flat surface may also affect transmission properties. The number of boards needed for each design can be found as follows:

$$
\frac{L_{\text {total }}}{N_{s}} \frac{1}{l_{\text {cell }}}=\frac{13 \mathrm{~cm}}{10 \text { stairs }} \frac{1 \text { layer }}{0.2366 \frac{\mathrm{cm}}{\text { board }}}=\frac{1.3}{0.2366} \approx 5 \frac{\text { boards }}{\text { stair }}
$$

Hence five $62 \mathrm{mil}$ etched FR-4 and five 31 mils spacer boards shall be needed per stair of the prism. There are 10 stairs, so 50 etched boards and 50 spacer boards will be needed for the prism structure.

It is envisioned that an order placed to a PCB manufacturer shall include more than the minimum requirements calculated above. With an extra amount of boards, spacing may be varied to lower or increase the resonant frequency of the structures. If the spacer boards are removed, more etched PCB boards are needed to fill the spaces and create a full size prism or transmission slab structure. 


\section{Chapter 10}

\section{Test setup}

\subsection{Test Setup in Anechoic Chamber}

Testing is conducted in the Anechoic Chamber at Cal Poly San Luis Obispo. Absorbent foam lines the chamber walls and reduces spurious reflections which may impact data if testing occurs in a regular environment such as a lab or classroom. The chamber is designed to accomodate far-field and radiation pattern testing. The positioner system allows for radiation pattern testing and is used to conduct refraction testing for a metamaterial prism. The Antenna lab has several horn antennas for use in free-space testing within the chamber. Both Rx and Tx antennas are used in the experiments. Two NARDA $641(7.05-10 \mathrm{GHz})$ horn antennas are used for initial testing in the X-band frequency range. A basic block diagram of the anechoic chamber apparatus is presented in Figure 10.1. 


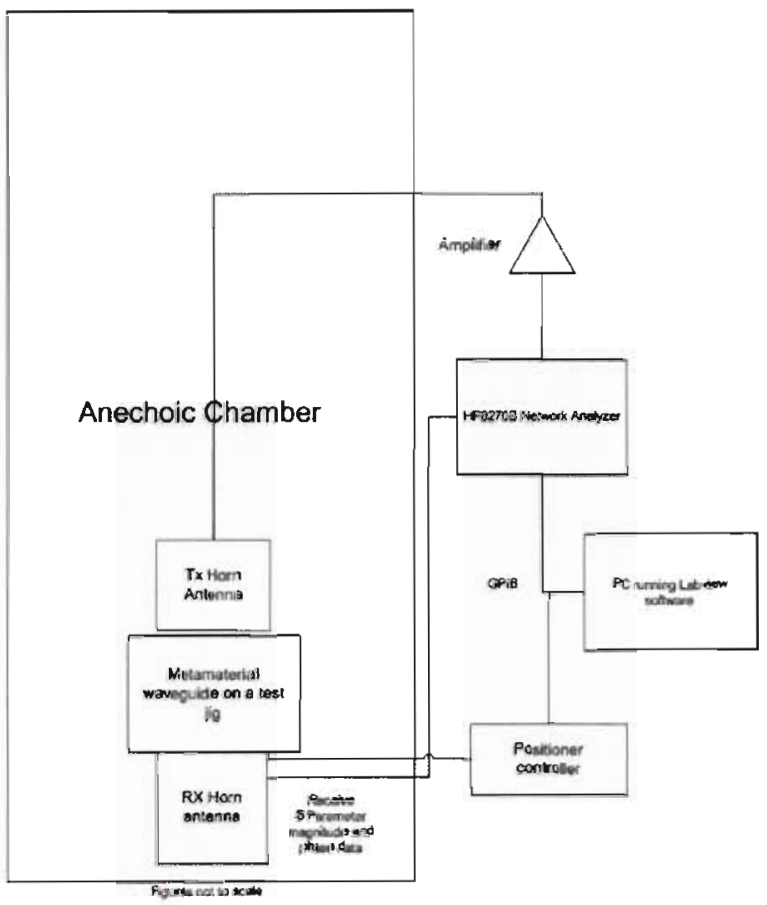

Figure 10.1 Block diagram for proposed testing of DNG metamaterials in the Anechoic Chamber.

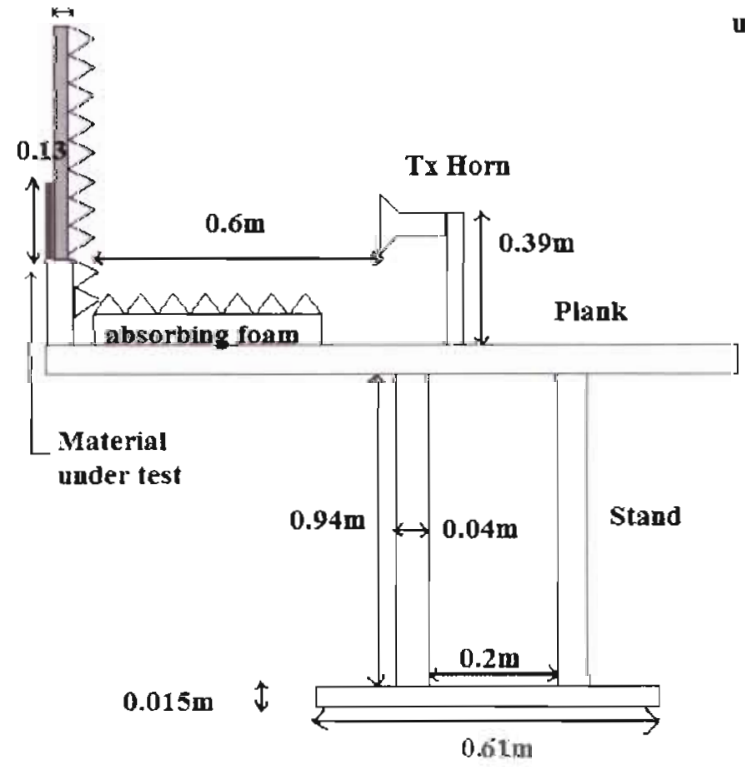

units in meters

Not to scale

Figure 10.2 Sideview of testsetup structure. 


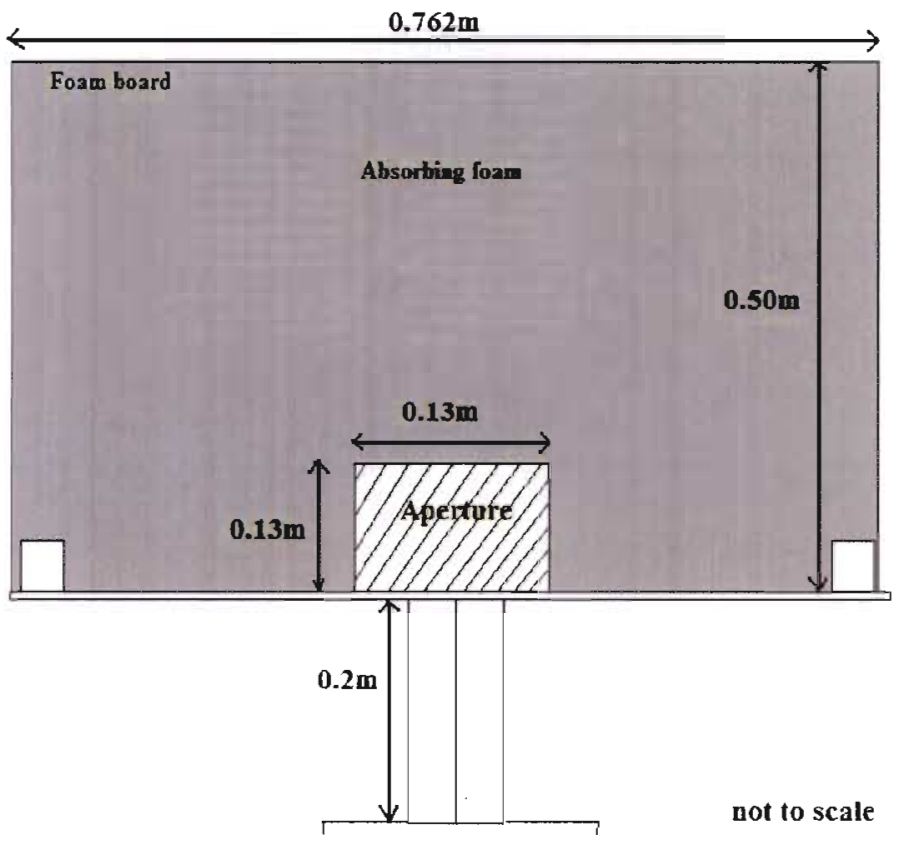

Figure 10.3 Foam board dimensions and square aperture for metamaterial slab placement. Metamaterial sample is embedded directly within an aperture in the foam board.

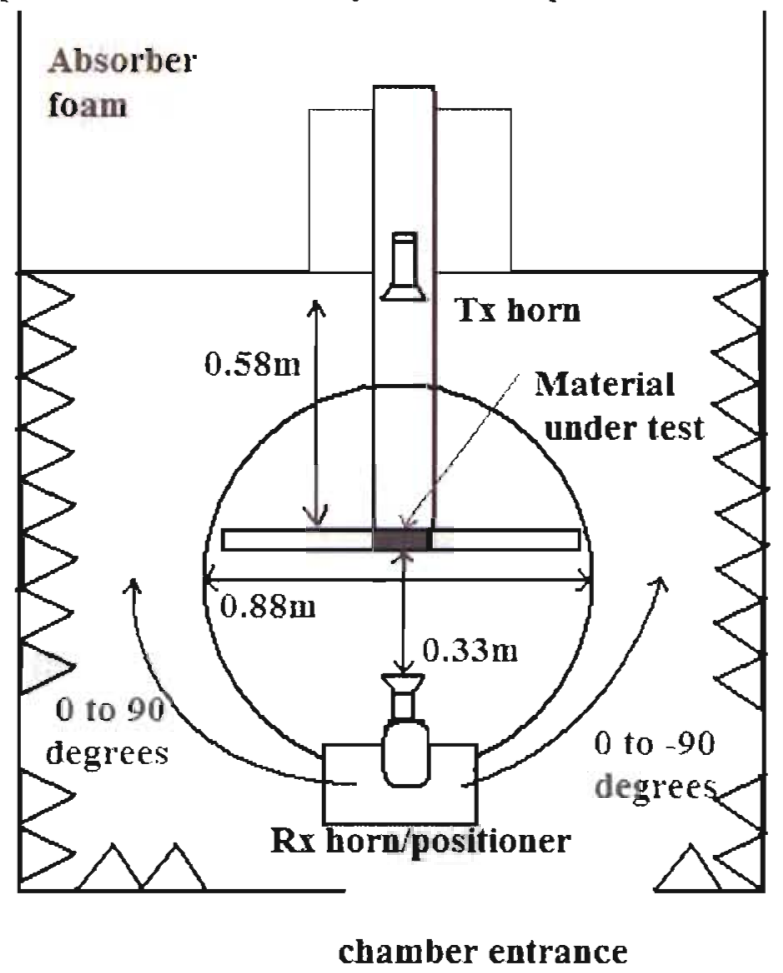

Figure 10.4 Top down view of antenna chamber looking specifically at the test and positioner setup. 
The test configuration is constructed with wood. As visible in Figure 10.2, the structure is supported with 2 legs. A plank is needed to allow the material under test to be placed closer to the receive horn. Furthermore, in order to obtain accurate refraction readings in the prism test, the metamaterial refracting plane must be placed over the rotation axis of the positioner. When the positioner rotates, the receive horn always faces the center of the metamaterial refraction plane. To minimize scattering and limit transmission detected by the receive horn to only the radiation passing through the metamamterial, a foam board is constructed as shown in Figure 10.3. The front face is covered with abosorbent foam to attenuate unwanted transmission and to minimize scattering and reflections. A $13 \mathrm{~cm} \times 13 \mathrm{~cm}$ square is left open leaving space for the metamaterial under test to be placed. Furthermore, foam is placed along the plank as shown in Figure 10.2 to limit transmission between $\mathrm{Tx}$ and $\mathrm{Rx}$ horns to the radiation passing through the metamaterial. The impact of adding the foam board and absorber foam to the setup is discussed in a section 10.2. A picture showing the built setup placed in the Anechoic chamber is shown in Figure 10.10.

\section{Determination of far field region and TX Horn antenna placement}

The maximum operating frequency is $10 \mathrm{GHz}$. The far field distance to ensure a plane wave is incident on the metamaterial is calculated as follows:

$$
\text { Far field distance }=\frac{2 d^{2}}{\lambda}
$$

Therefore, the minimum distance between the transmit horn and the metamaterial to ensure an illuminating plane wave at $10 \mathrm{GHz}$ is $58 \mathrm{~cm}$.

\section{Horn Antenna beamwidth}

The H-plane beamwidth of the Narda 641 horn antenna based on the datasheet is 30 degrees. The plane of the metamaterial is located $60 \mathrm{~cm}$ from the transmit horn. Radiation extends as far as $33 \mathrm{~cm}$ $\left(60 \tan \left(30^{\circ}\right)\right)$ out from the center of the metamaterial test plane. 


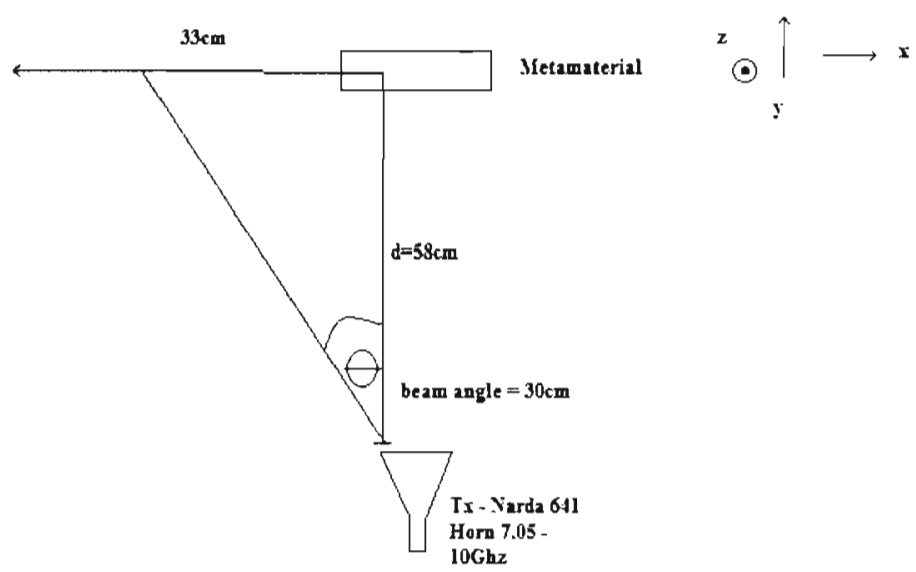

Figure 10.5 Horn antenna placed at the minimum distance to generate a plane wave. The maximum extent of the radiation due to a beam angle of $30 \mathrm{~cm}$ is shown to be $33 \mathrm{~cm}$.

\subsection{Test Setup characterization}

To examine the free-space test setup in the Anechoic chamber prior to metamaterial characterization, the test fixture is placed in the chamber. Network analyzer calibration ( $3.5 \mathrm{~mm}$ kit) compensates for the long coax cables extending into the chamber. Calibration is performed over the range from $7 \mathrm{GHz}$ to $10 \mathrm{GHz}$. The effect of the cable lengths and connectors must be accounted for frequencies within the specified range. Note that the initial results presented below were produced after calibrating the network analyzer with a $3.5 \mathrm{~mm}$ cal kit and then connecting the coax cables to the horn antennas with an SMA to N-type adapter.

Transmission and reflection testing between the two horn antennas in Figure 10.4 is conducted with the foam screen removed. The foam screen is used to absorb radiation not in a direct path from the Tx to the Rx. Test results for the the slab and foam removed are show in Figure 10.6. Through the addition of the screen and absorber foam lining the wooden plank parallel to the direction of wave propagation, transmission increases by 2 or $3 \mathrm{~dB}$ throughout the test frequency range according to 10.7. Furthermore, the addition of the foam reduces reflections by 3 to $4 \mathrm{~dB}$ from 7 to $8.5 \mathrm{GHz}$, while being approximately the same in the foamless case throughout the remaining frequency range. An additional test of the foam board effectiveness is to block the aperture with absorbing foam. 
As expected, $\left|S_{21}\right| \mathrm{dB}$ decreased to approximately $-50 \mathrm{~dB}$ at $8.5 \mathrm{GHz}$ while the scattering parameter $\left|S_{11}\right| \mathrm{dB}$ was largely unaffected with a low reflection reading of $-35 \mathrm{~dB}$ at $8.5 \mathrm{GHz}$. Finally, the foam board is replaced by sheet metal. It can be seen in Figure 10.6 that reflections increase with the addition of a metal sheet from $-30 \mathrm{~dB}$ to $-18 \mathrm{~dB}$.

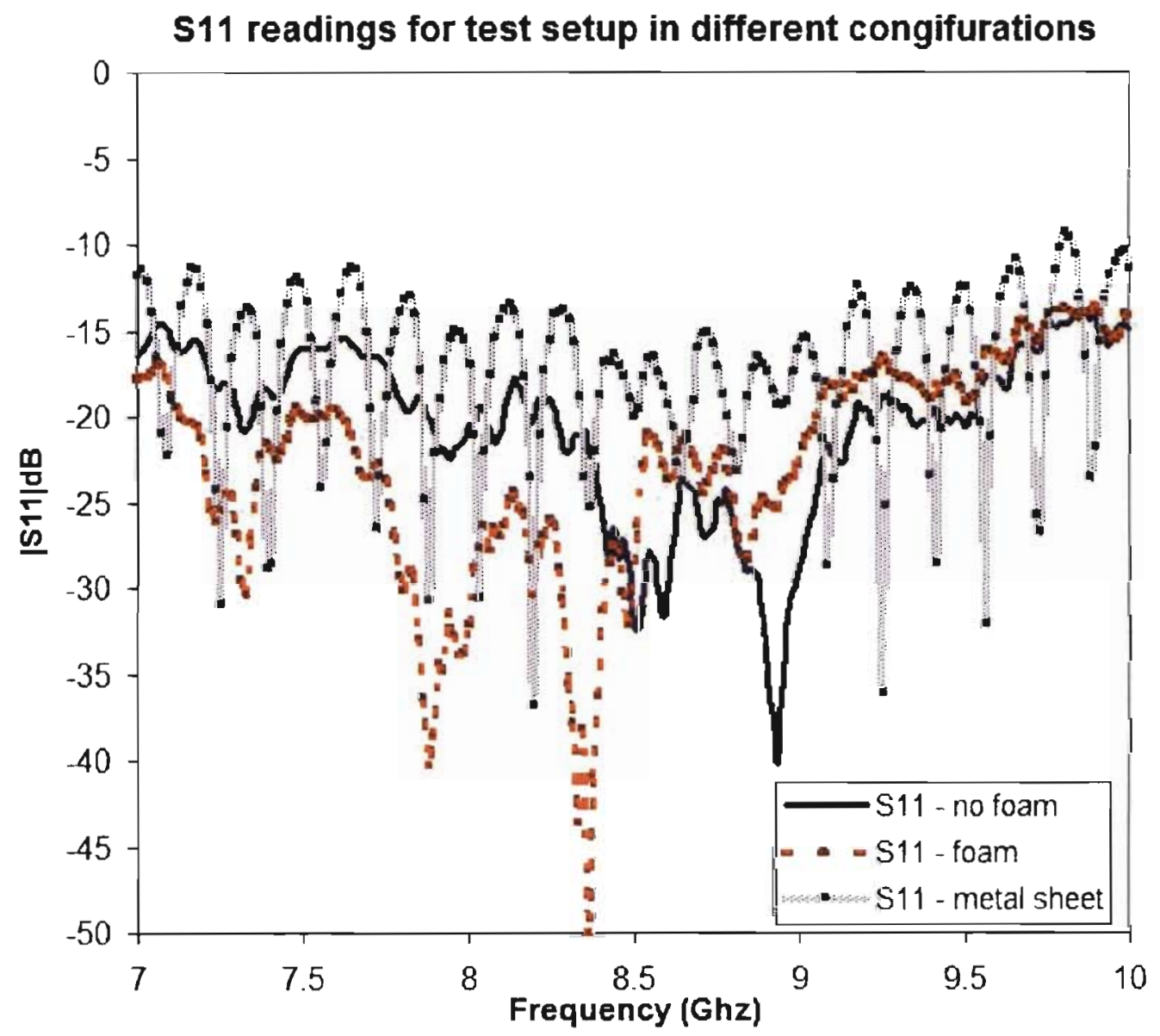

Figure 10.6 Reflection readings for test fixture without foam board and absorbers, with foam board/absorbers and with metal sheet in incidence path. 
$\mathbf{S 2 1}$ readings for 2 test setups

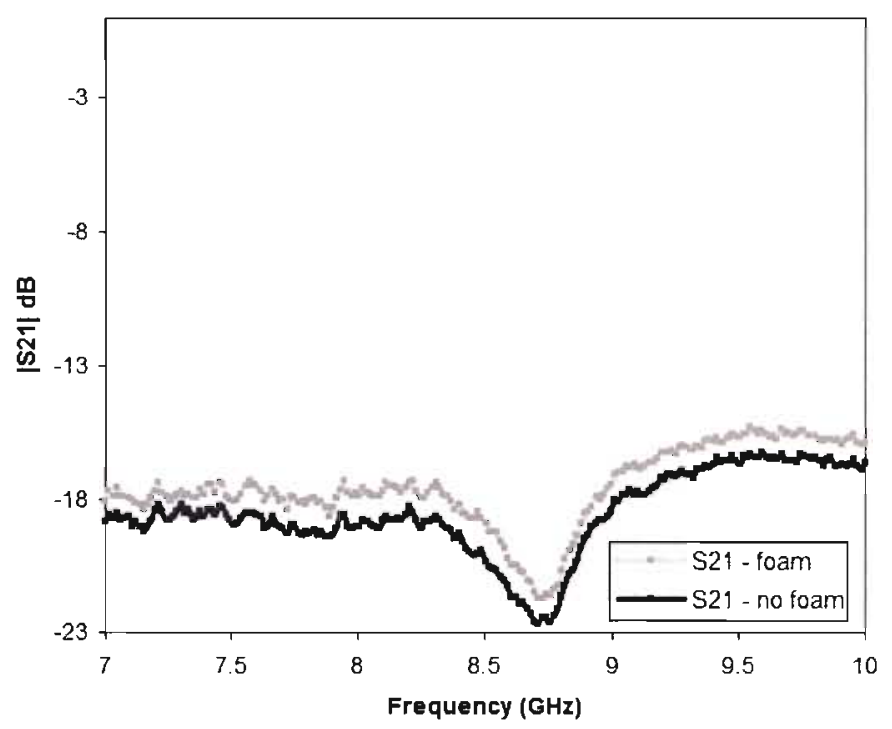

Figure 10.7 Transmission measurements in free space with no metamaterial slab. Plots for setup with/without foam board and absorbers.

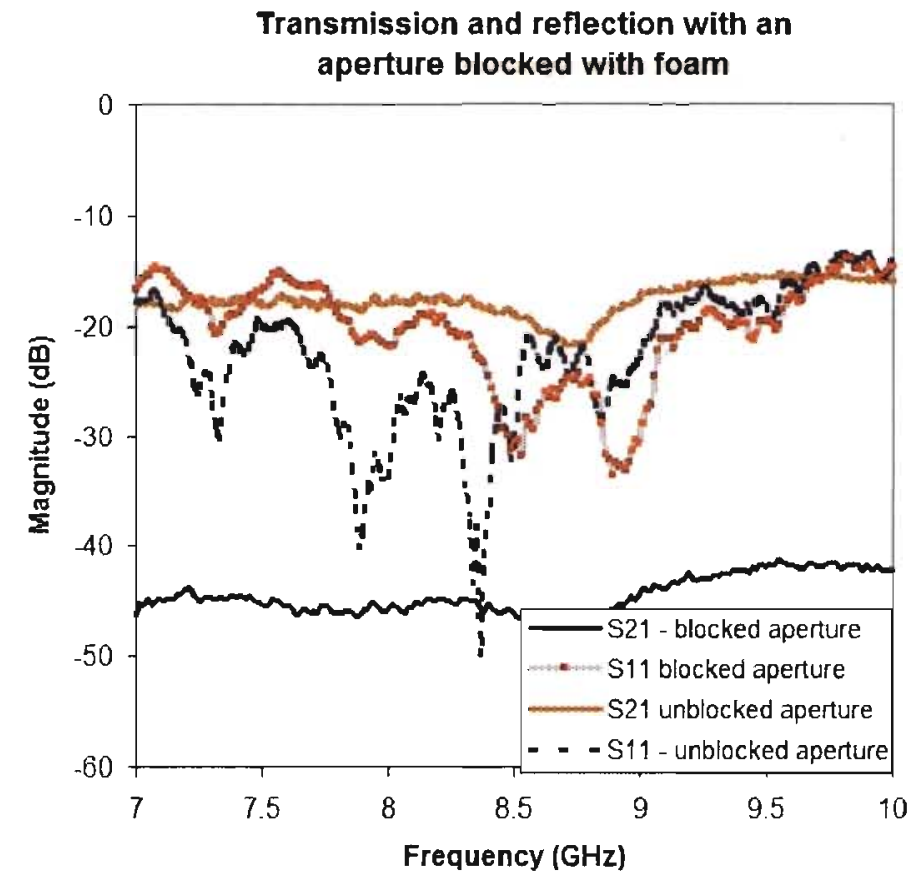

Figure 10.8 Transmission and reflection with square aperture in foam board blocked. 


\section{Normalized transmission calibration for free-space testing}

The HP8270C Network Analyzer available in the Cal Poly Antenna lab not only performs standard one port and 2 port calibration, but allows for fast calibration of cables connected to antennas such as the horn antennas used in this thesis. By performing a transmission calibration with the cables connected to the horn antenna, the test setup may be calibrated such that the power received by the receive hom with the test-setup and no object in the foam board's aperture represents OdB of total transmission. Hence, transmission has been normalized to the case of power received when no metamaterial sample is present on the test platform. Figure 11.14 shows that transmission after calibration is $0 \mathrm{~dB}$ throughout the frequency range of measurement and reflection is $<40 \mathrm{~dB}$. If a metal sheet is placed in the transmission path, transmission drops below -10dB and reflections denoted by $\left|S_{11}\right| d B$ approach $0 \mathrm{~dB}$.

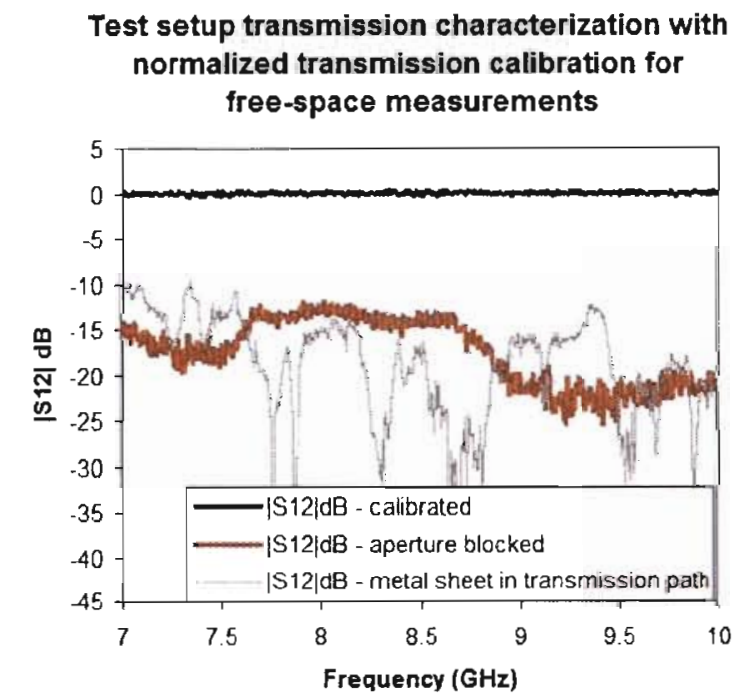

Reflection measurements after test setup normalized calibration for free-space measurement

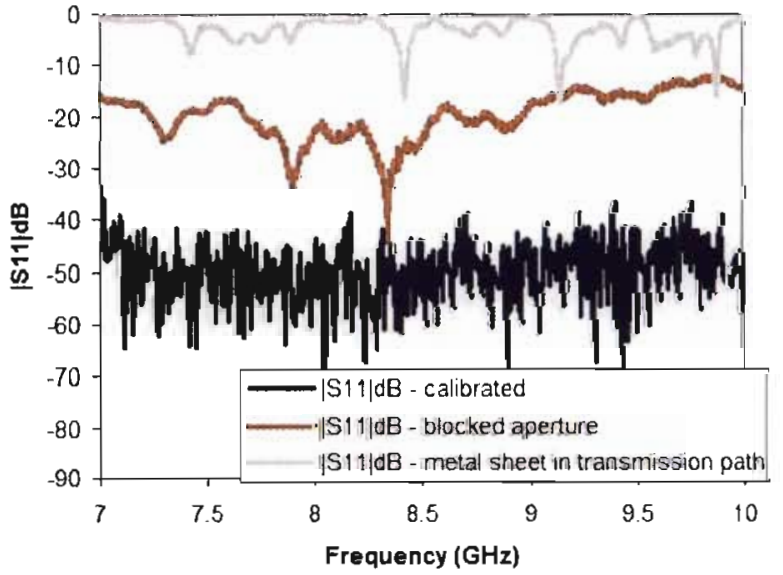

Figure 10.9 Transmission and reflection measurements to validate test setup after normalized transmission calibration for free-space testing. 


\subsection{Test plan}

\subsubsection{Transmission testing}

Transmission testing is conducted in the frequency range from $7 \mathrm{GHz}$ to $10 \mathrm{GHz}$ set by Narda 641 horn antenna frequency range. Increasing the range in the future corresponds to increasing the far-field distance; the transmit-receive horn distance must be increased. The HP 8720C Network Analyzer with a frequency range of $50 \mathrm{MHz}$ to $20 \mathrm{GHz}$ is available in the antenna lab for S-parameter measurements. $\left|S_{21}\right|,\left|S_{11}\right|,<S_{21}$ and $<S_{11}$ are recorded for 401 frequency points between 7 and $10 \mathrm{GHz}$

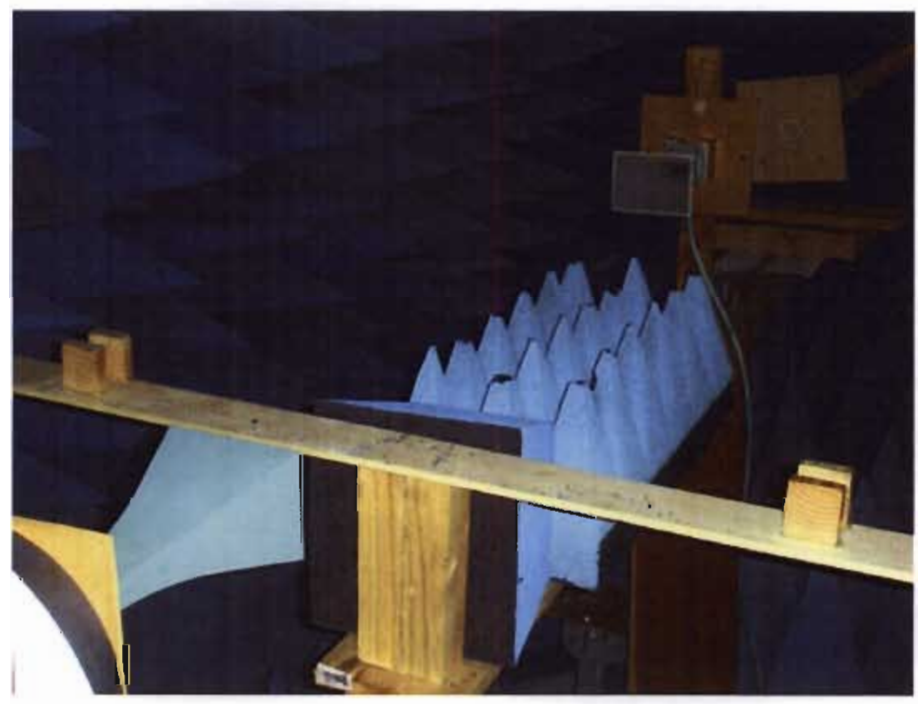

Figure 10.10 Test setup in Anechoic chamber. Foam board has been removed to make both $\mathrm{Rx}$ and $\mathrm{Tx}$ horn antennas visibile.

\subsubsection{Metamaterial refraction testing}

The fabricated metamaterial prism is placed on the test setup with the objective of trying to finding for a given transmission frequency, the azimuthal angle $(\phi)$ of the receive antenna that allows for maximum power received. For metamaterials, the permittivity, permeability and hence refraction index are frequency dependent. Therefore, by finding the azimuthal angle in the H-plane one can derive the index of refraction at that frequency using Snell's law. The azimuthal angle is varied from -90 degrees to 90 degrees as shown in Figure 10.4. The process may be conducted manually 
by inputting commands to the Sunsol Sciences controller SC104V. Additionally, previous projects in the Antenna and Microwave groups involved the development of LabView scripts to automate the radiation pattern testing process via GPIB. This would allow for more data points to be recorded in a shorter amount of time. 


\section{Chapter 11}

\section{Test results}

Transmission metamaterial slabs and prism shaped slabs for refraction testing have been fabricated in the lab after receiving a shipment of PCB boards with specifically designed copper etched inclusions.

\subsection{Transmisson testing}

\subsubsection{S-SRR metamaterial}

The metamaterial slab was placed in the test setup previously described. Illuminated by a $7-10 \mathrm{GHz}$ standard gain horn, the transmission properties are measured with an identical standard gain receive horn on opposite side of the absorbing foam board. The first figure compares transmission and reflection S-parameters measured in the anechoic chamber with those simulated in Ansoft HFSS previously using a PEC/PMC waveport configuration. The peak transmission frequency and the effect of capacitive coupling are underestimated slightly by HFSS as can be seen with an expected peak in S-parameter resonance at $8.5 \mathrm{GHz}$ actually being $8.3 \mathrm{GHz}$.

In order to tune the metamaterials, only a few options are available. The PCB boards may be cut to size the metamaterial. Furthermore, the spacing between etched PCB boards may be altered by increasing or decreasing the spacer board thickness. The spacer boards are unclad FR-4 board like the PCB boards and hence have a dielectric permittivity of $\epsilon_{r}=4.4$. Either the thickness of the 


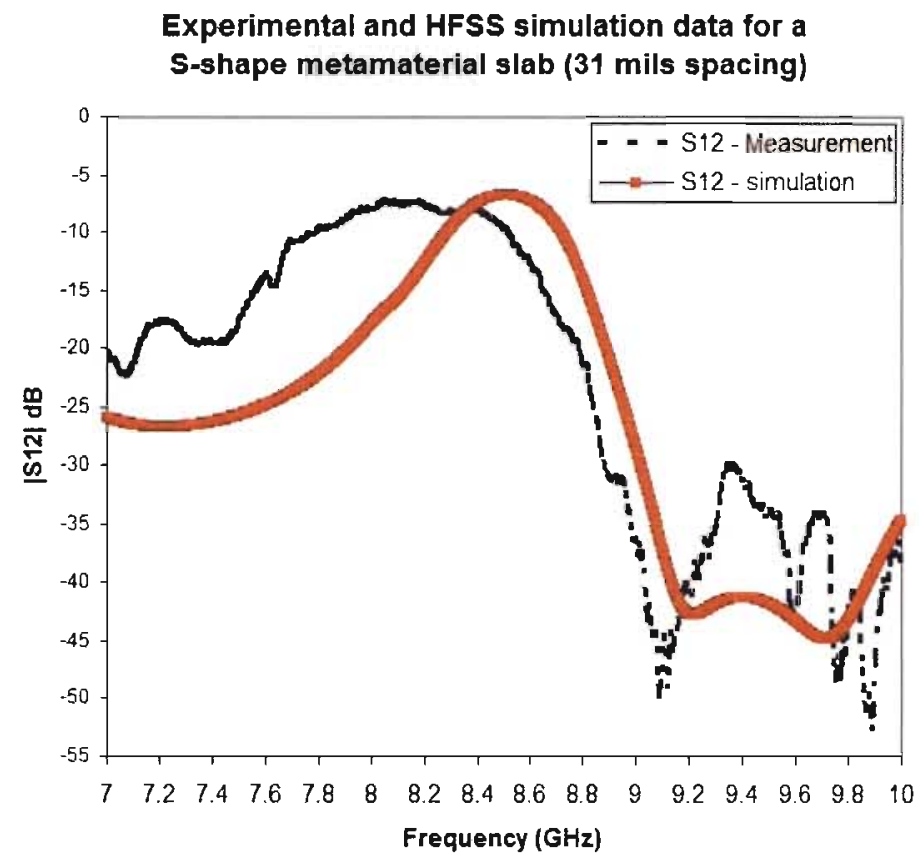

Figure 11.1 Measured slab transmission data compared to theoretical data predicted by HFSS.

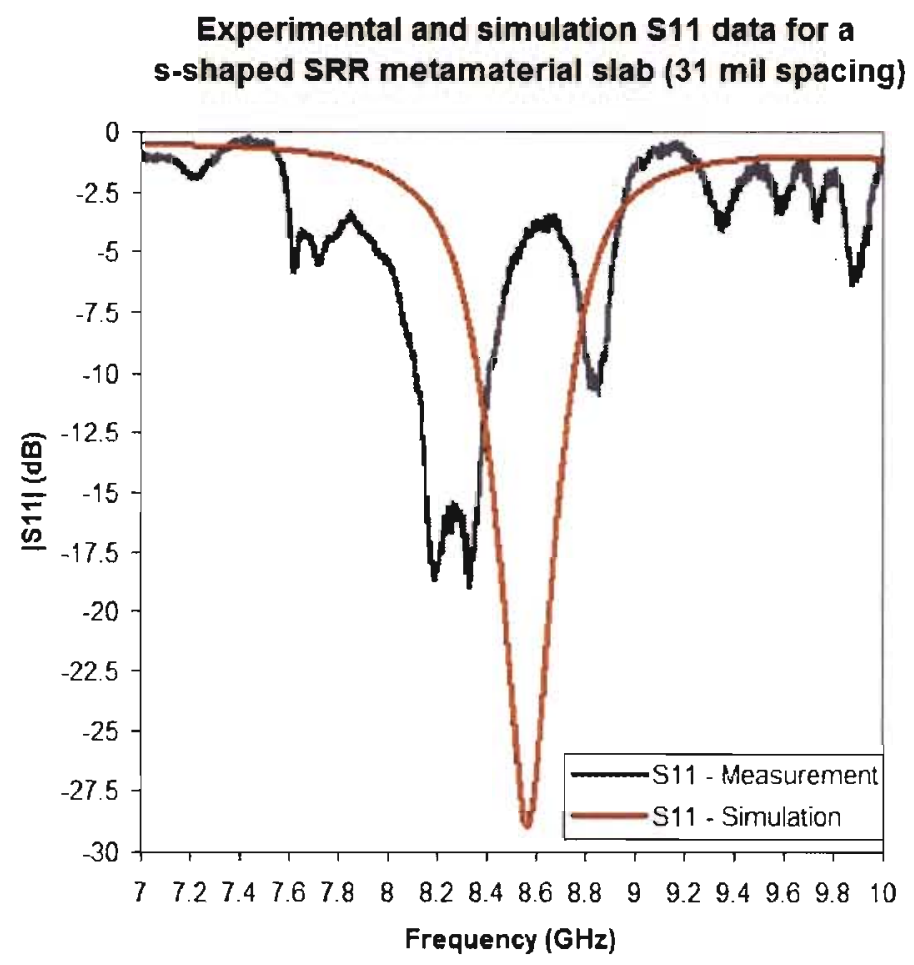

Figure 11.2 Measured slab reflection data compared to reflection data predicted by HFSS. 
spacer boards may be altered or a number of thinner boards may be added. The graph below shows the effect in transmission when increasing the spacing thickness between copper etched boards from $31 \mathrm{mil}$ to $62 \mathrm{mil}$. The resonant frequency where a peak in transmission is apparent increases from approximately $8.3 \mathrm{GHz}$ to $8.8 \mathrm{GHz}$. This is to be expected as resonant frequency is related to the basic equation $\omega_{0}=\frac{1}{\sqrt{\bar{L}}}$. The capacitive coupling between adjacent unit cells in the H-plane decreases as the distance between unit cells is increased. Decreasing $\mathrm{C}$ serves to decrease the value of the denominator and hence increases the resonance frequency.

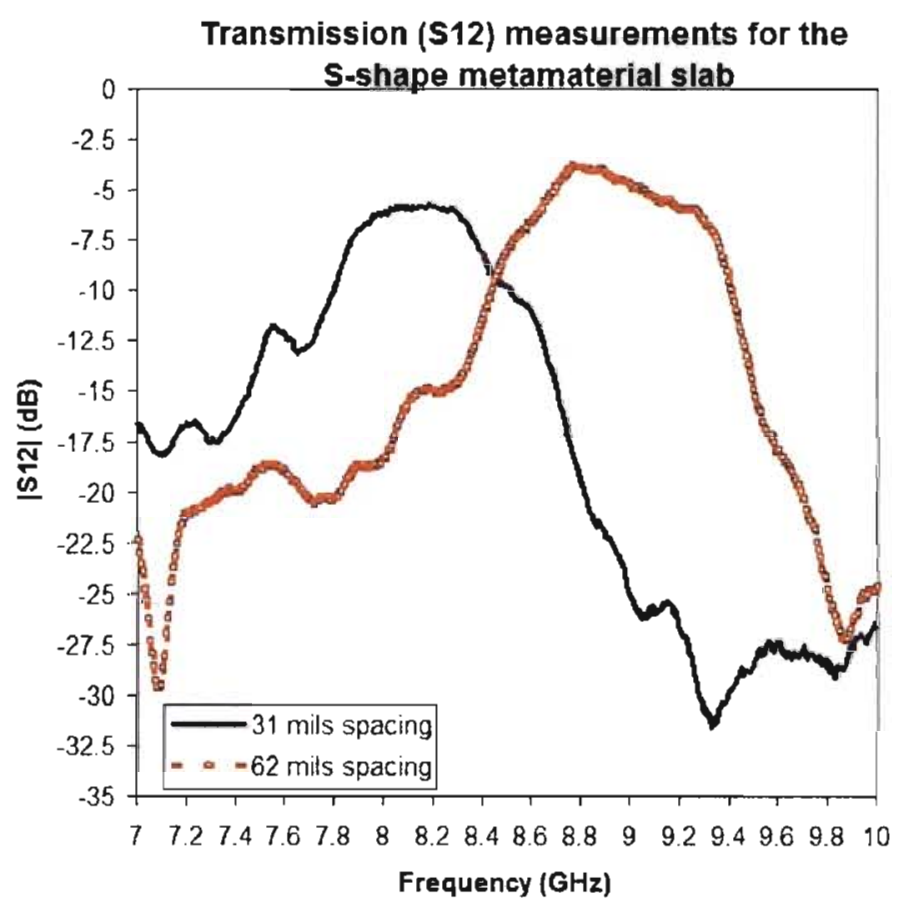

Figure 11.3 Graph showing the frequency shift in the transmission peak when changing the thickness of 31 mil spacer boards to 62 mils.

Using data recorded by the Vector Network Analyzer, and exporting it to a text document, it is possible to perform parameter extraction via a modified version of the Nicolson Ross Weir (NRW) method. This method was examined in detail in an earlier status report and a Matlab script was written to read in data and perform the parameter extraction. It was previously shown in the parameter extraction chapter that extracted divergences in permeability and permittivity correspond closely to resonance in the transmission and reflection parameters. (How was this code verified? Show example calculations on analytically known configurations - from research papers?) Using 
magnitude and de-embedded phase data for $\mathrm{S} 11$ and $\mathrm{S} 12$ or $\mathrm{S} 21$, it is possible to numerically calculate the relative permeability, permittivity and index of refraction for the $S$-shaped metamaterial transmission slab.

Figure 11.4 shows that the resonance in permeability and permittivity as extracted from measurement data is lower than that of simulated data by approximately $0.5 \mathrm{GHz}$. This corresponds with the shift in measured resonance peak in the transmission parameters with respect to HFSS simulated values. Of note, is the large amount of noise and fluctuation in the extracted measurement results of Fig 3 compared to the extracted bulk parameters from HFSS simulations. This may be attributed to the noise clearly visible in the phase measurements for the S-parameters. Figure 3 shows a strong negative permeability and permittivity response from $9.2 \mathrm{GHz}$ to $9.8 \mathrm{GHz}$. This is not expected. This response is due to the sensitivity of the extraction method to phase variations. De-embedded phase information for the transmission and reflection S-parameters clearly show unexpected phase fluctuations in this frequency range. The extraction method equations are particularly sensitive to noise and fluctuations in the phase information for both S12 and S11. The rubber bands are not the cause of the phase variations as they are wrapped around the boards on the top and bottom faces in such a manner that the illuminating wave is not incident upon them. Other options for holding the boards together were considered. The use of glue for bonding the boards will negate the need for bands however, the structures can no longer be easily modified. Drilling holes and placing rods through the structure is also a viable option. However, unless rods of the same permittivity are used, the wave will encounter a material boundary where refraction and dispersion may occur unlike with the use of rubber bands not in the immediate propagation path. The extracted index of refraction for both simulated and measured cases may be seen in figure 4 . Again, a visible shift in frequency is seen in the index of refraction for measured data as apposed to simulated data. For the measured data, extracted results show negative refraction is obtained in the $\mathrm{S}$-shaped transmission slab from $7.6 \mathrm{GHz}$ to $8.4 \mathrm{GHz}$. 


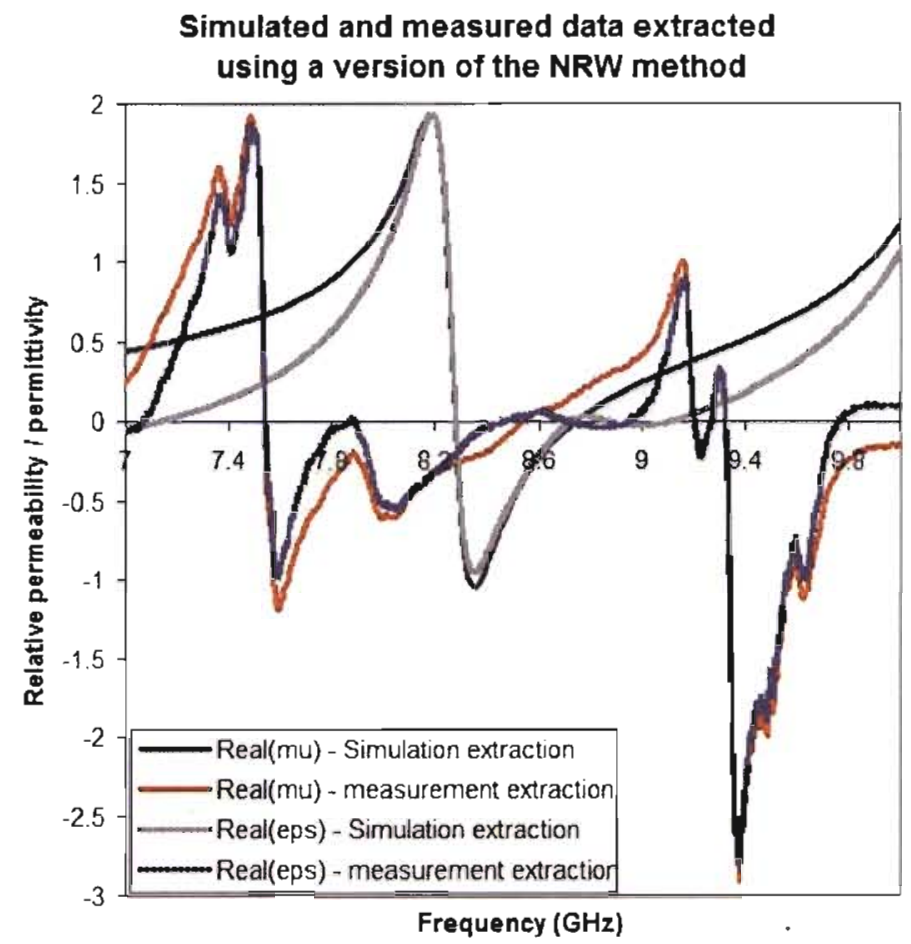

Figure 11.4 Extracted relative permeability and permittivity for the $S$-shaped transmission slab.

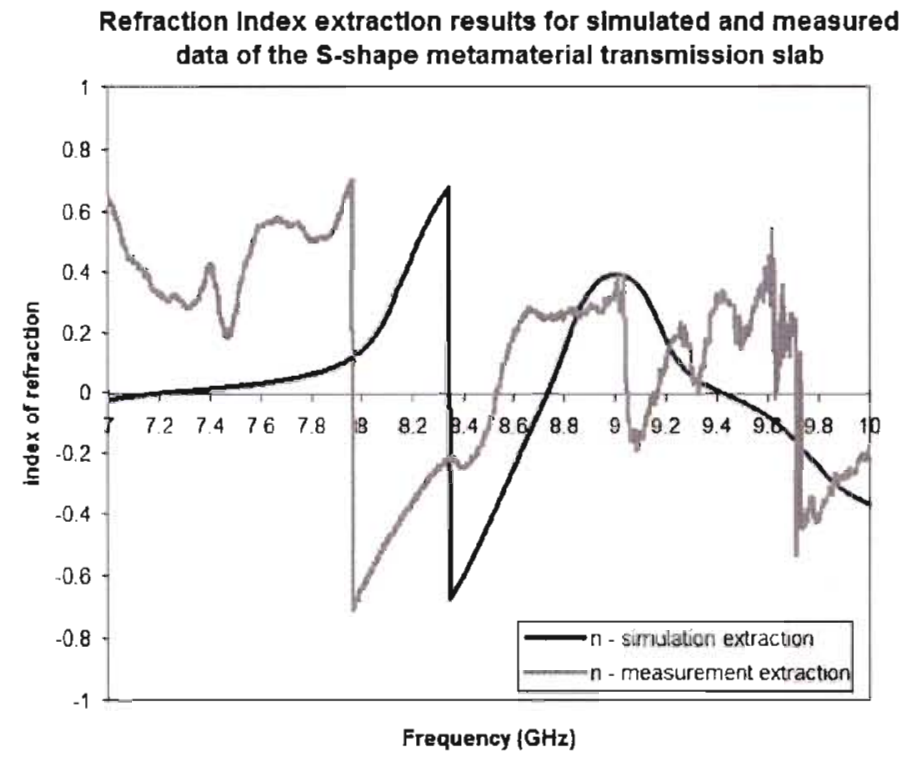

Figure 11.5 Extracted index of refraction for the S-shaped transmission slab. 


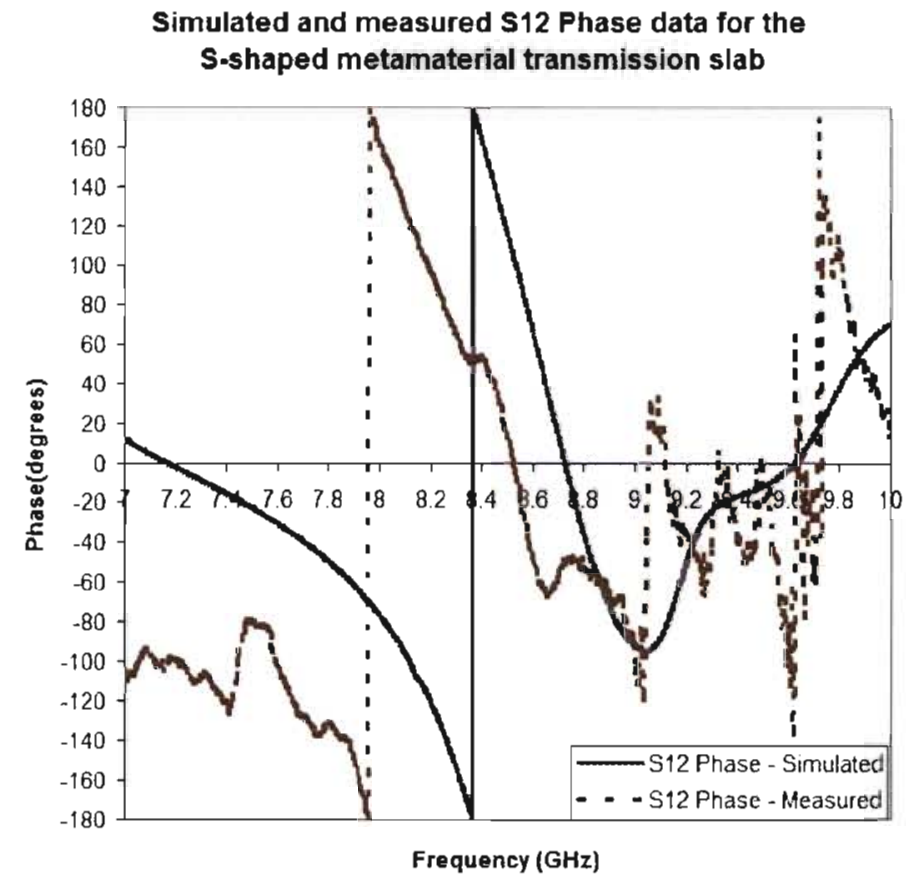

Figure 11.6 Simulated and measured S12 phase data. The measured data exhibits phase noise in the range from 9 to $9.8 \mathrm{GHz}$.

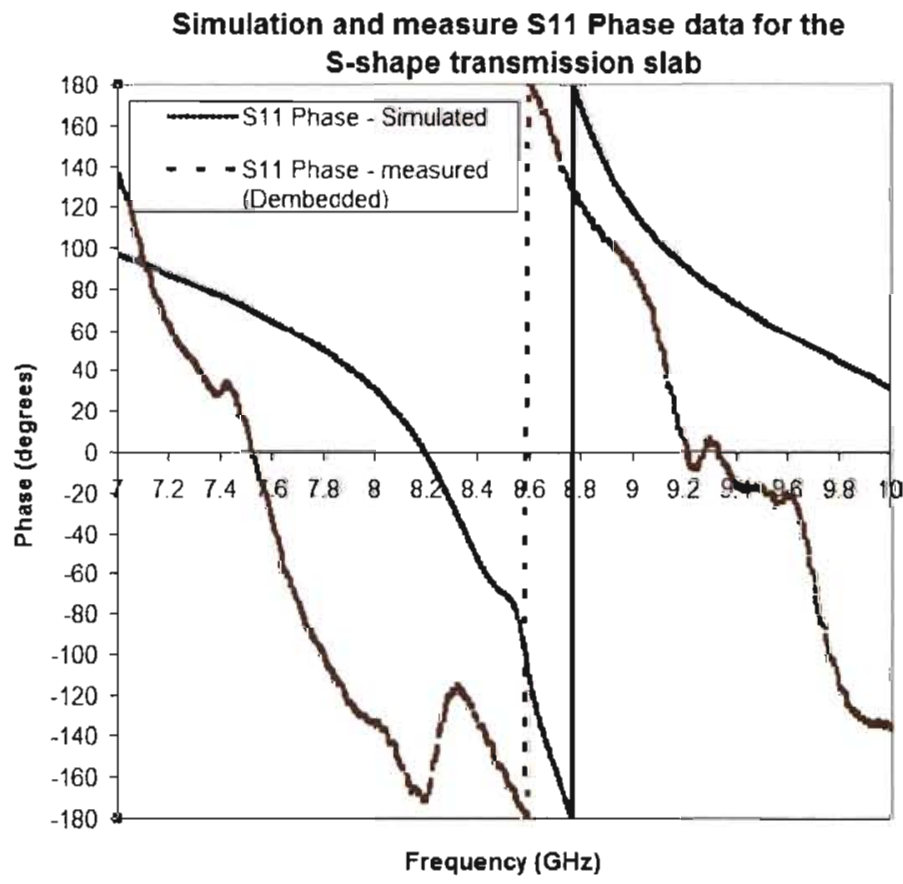

Figure 11.7 Simulated and measured $\mathrm{S} 11$ phase data. 


\subsubsection{SRR/CLS structure}

The SRR/CLS structure produced results inconsistent with the simulated data. As can be seen, within the Narda Horn bandwidth of 7 to $10 \mathrm{GHz}$, a simulated resonance in $8.2 \mathrm{GHz}$ to $8.4 \mathrm{GHz}$ is visible. However, upon fabrication and testing of the equivalent SRR/CLS metamaterial structure, the peak resonance is obtained at $9.9 \mathrm{GHz}$ as seen below. Furthermore, the resonance characteristics as compared to the S-SRR structure in both simulations and measurement show a resonance whose peak transmission is at approximately $-20 \mathrm{~dB}$. The S-SRR structure discussed above shows a peak transmission of $-5 \mathrm{~dB}$. Solving for the linear magnitudes of both transmission and finding their relative ratio shows that at the resonance frequency, approximately 25 times the signal power transmitted by Tx horn is received by the Rx horn for the S-SRR unit cell compared to the SRR/CLS unit cell. Hence, the S-SRR metamaterial slab achieves closer matching to free space.

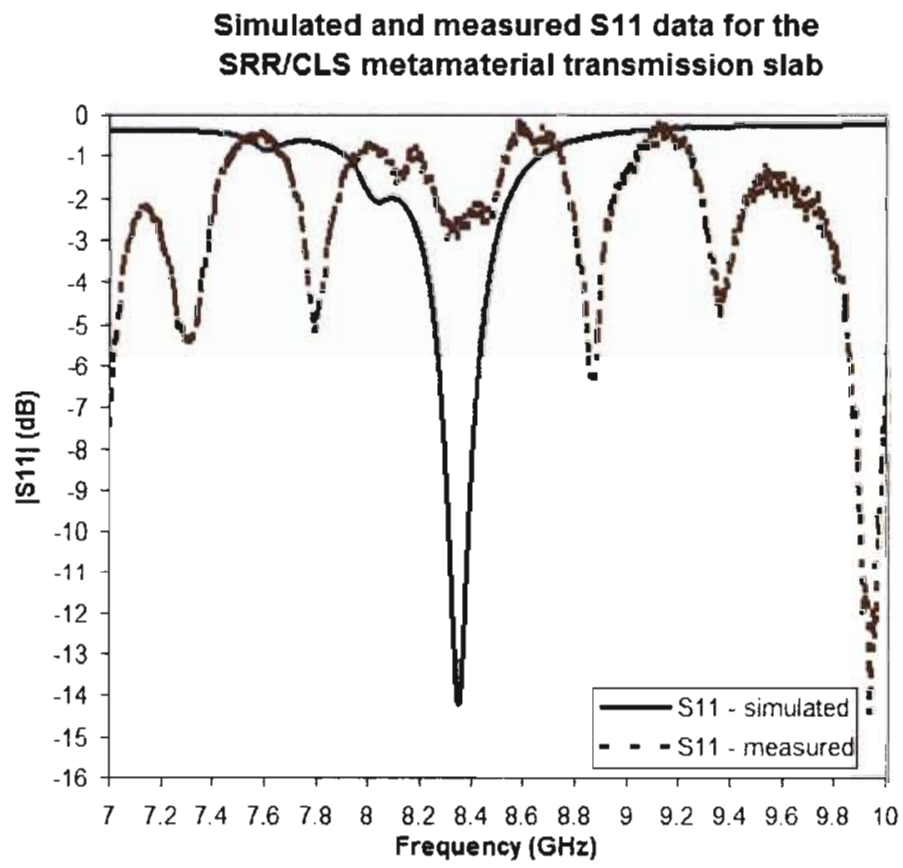

Figure 11.8 Simulated and measured insertion loss (S11) data for SRR/CLS metamaterial slab 


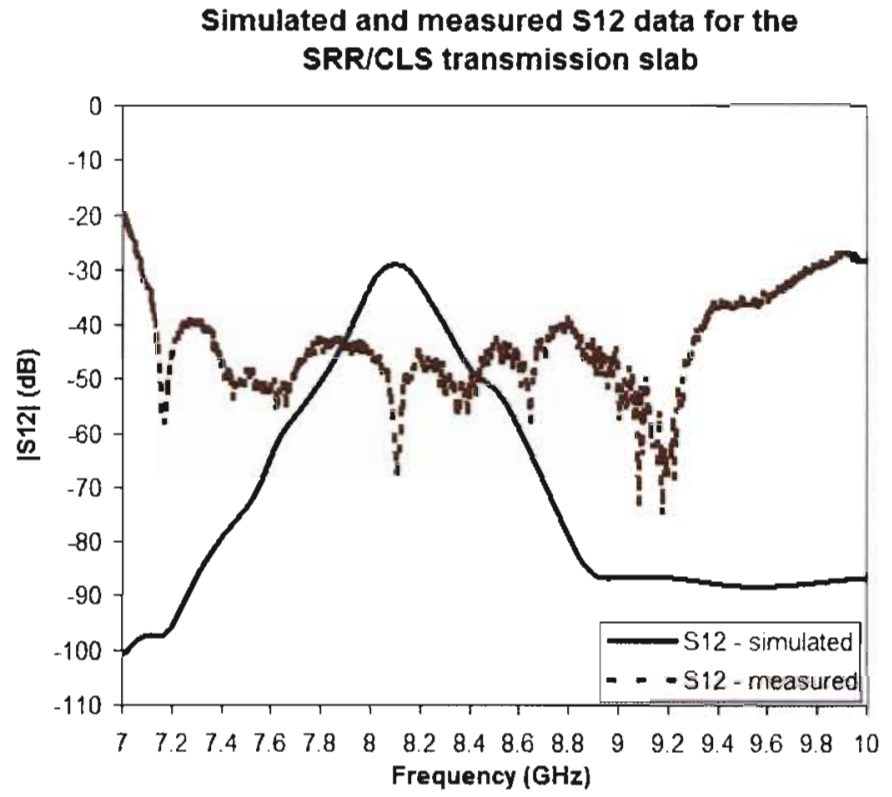

Figure 11.9 Simulated and measured (S12) data for SRR/CLS metamaterial slab.

\subsection{Metamaterial prism refraction testing}

The prism design as previously described in a previous status report has been fabricated using etched PCB boards. Initial testing involved a $13 \mathrm{~cm} \times 13 \mathrm{~cm}$ square aperture in the foam board. Hence, the prism metamaterial was $13 \mathrm{~cm} \times 13 \mathrm{~cm}$ in the plane of the $\mathrm{Tx}$ and $\mathrm{Rx}$ horn aperture. As can be seen in the test plan diagrams, the distance between the metamaterial sample under test and the Rx horn antenna is approximately $30 \mathrm{~cm}$. The accuracy of azimuthal radiation patterns at a distance within the nearfield of the radiating prism metamaterial is dependent on the size of the aperture in the absorbing foam board and the Rx horn aperture size in the Cross-pol direction. The metamaterial as described in the test plan is in the far-field of the transmit horn. The receive horn distance from the metamaterial however is limited to the diameter of the positioner system. A larger diameter would allow the receive horn to be placed further away from the metamaterial and more sensitive to the peak transmission angle. The beam width detected by the Rx horn can be reduced for more accurate readings by reducing the aperture in the foam board to be $13 \mathrm{~cm} \times 6 \mathrm{~cm}$. The $6 \mathrm{~cm}$ is in the direction of the radiated H-field. Furthermore, an X-band HP X890A Standard gain waveguide horn 


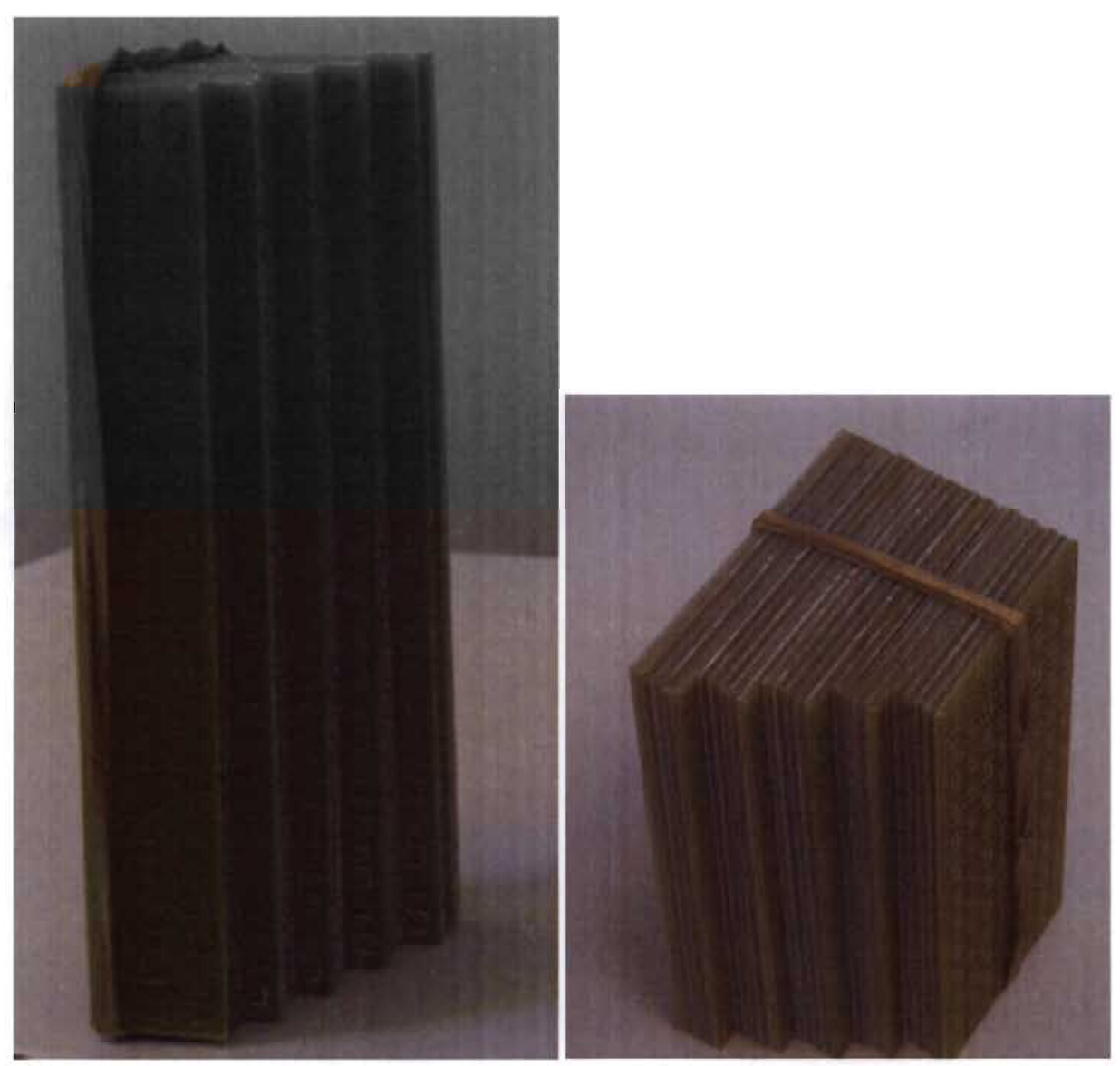

Figure 11.10 Fabricated metamaterial prisms with S-SRR inclusions 
replaced the receive Narda horn. The horn aperture size is reduced from the $9.3 \mathrm{~cm}$ to $2.0 \mathrm{~cm}$ in the H-direction.

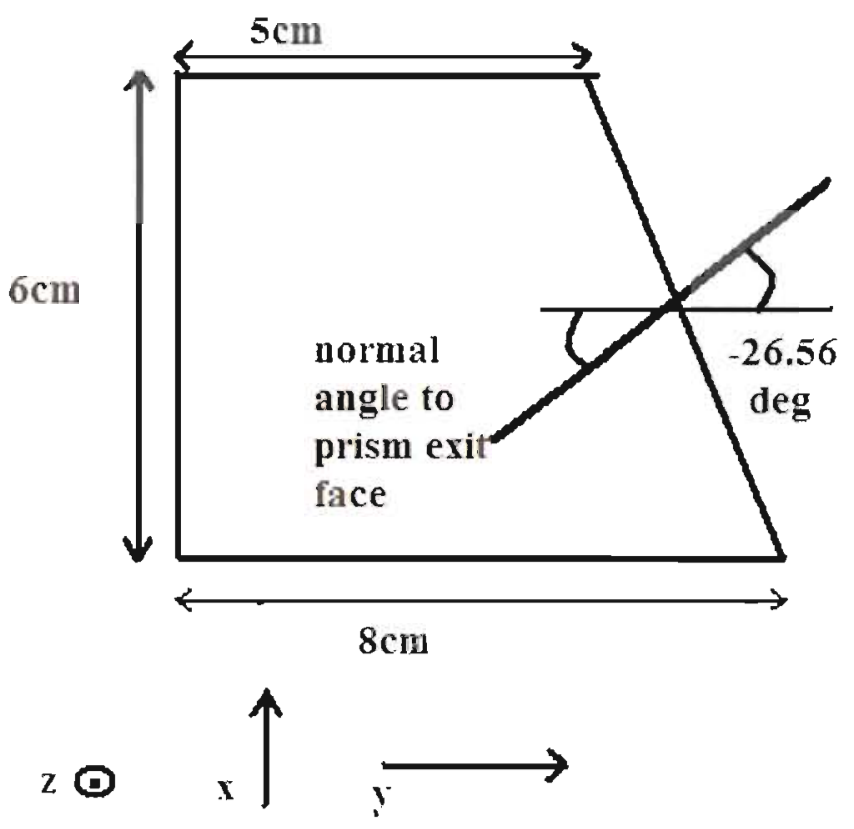

Figure 11.11 Theoretical diagram of prism metamaterial sample with modified dimensions. The normal angle is 26.56 degrees. All azimuthal measurements are taken with this normal angle taken as 0 degrees.

Azimuthal sweeps are conducted in the anechoic chamber. For each frequency point in the range of interest ( 7 to $10 \mathrm{GHz}$ ), the azimuthal angle that produces a peak in transmission is found. This angle in the phi direction is converted to an angle referenced to the normal angle on the exit face of the prism. This peak transmission angle with respect to the normal is substituted into Snell's law to obtain the index of refraction of the metamaterial prism.

Figure 11.13 shows the calculated index of refraction by finding the peak location in transmitted power. Negative refraction indicated by a negative value for $\mathrm{n}$ is seen from 8.2 to $9 \mathrm{GHz}$. Note that a gap in measurements is visible from $7.9 \mathrm{GHz}$ to $8.2 \mathrm{GHz}$ and $9 \mathrm{GHz}$ to $9.2 \mathrm{GHz}$. In these frequency ranges, peak power distribution oscillated between right handed and left - handed behavior making it difficult to reasonably estimate the index of refraction by employing the method of finding peak angle phi, for each frequency point. Thus, in these regions the index of refraction is unknown. Figure 11.14 plots two separate frequencies that exhibited right-handed and left-handed behavior. 


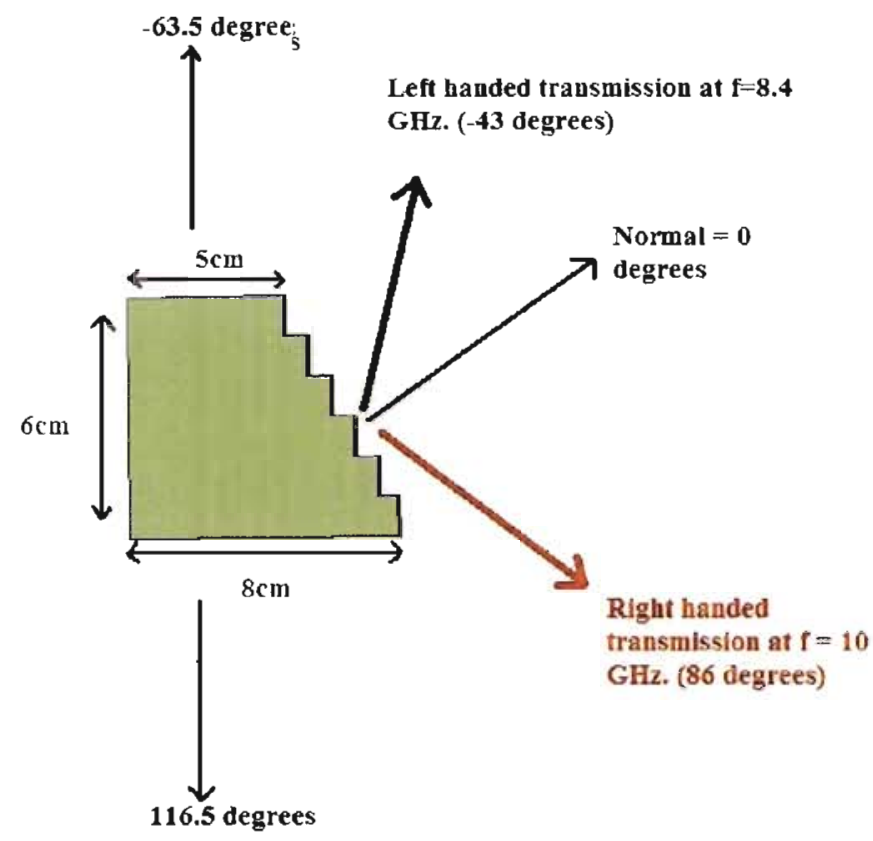

Figure 11.12 Top down view diagram of modified prism. Diagram indicate reference normal angle and directions of peak radiation for 2 frequencies, one with left hand wave propagation and the other showing right hand wave propagation.

The plots show the normalized readings for $\left|S_{12}^{2}\right|$, hence the angle at which peak power transmission is received is indicated with a power received value of 1.0 . Thus results are normalized with respect to the peak power detected by the receive horn during an azimuthal sweep at given frequency. At $10.4 \mathrm{GHz}$, the majority of the power is transmitted to 86 degrees from the prism normal. ), indicative of right-handed behavior. On the other hand, at $8.4 \mathrm{GHz}$, a peak in transmission is seen at -43 degrees from the prism normal direction. Due to refraction in the opposite direction from the normal, it may be concluded that the prism exhibits left-handed or negative refractive behavior at this frequency. 


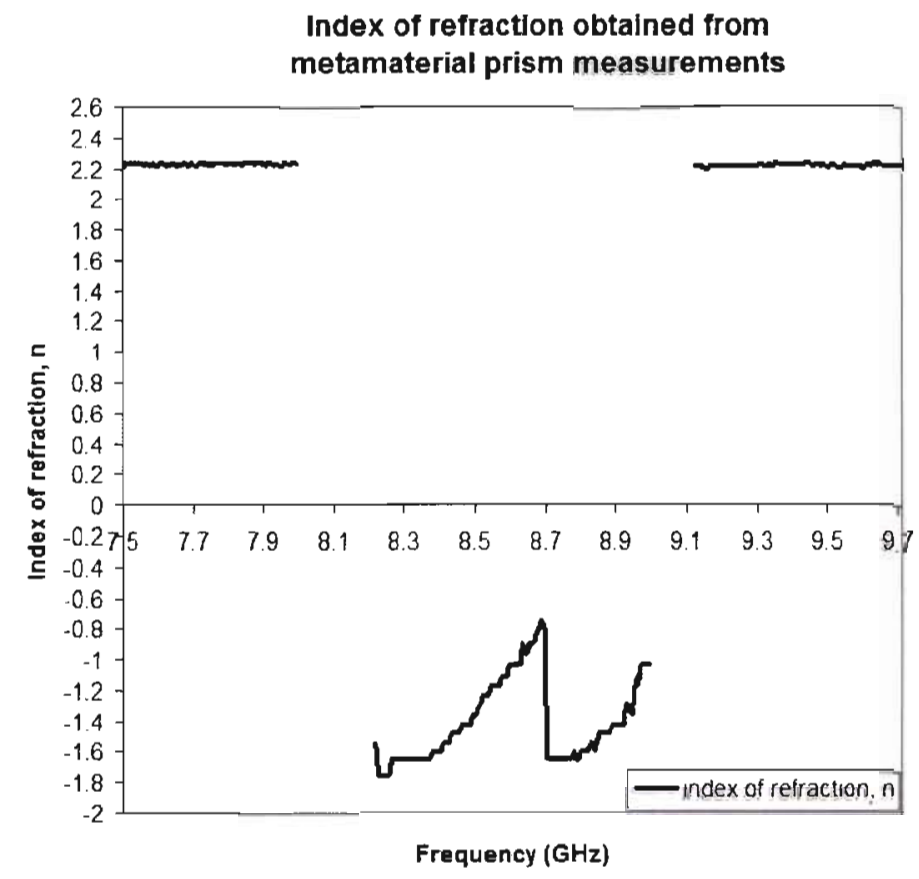

Figure 11.13 Index of refraction obtained from prism experiment.

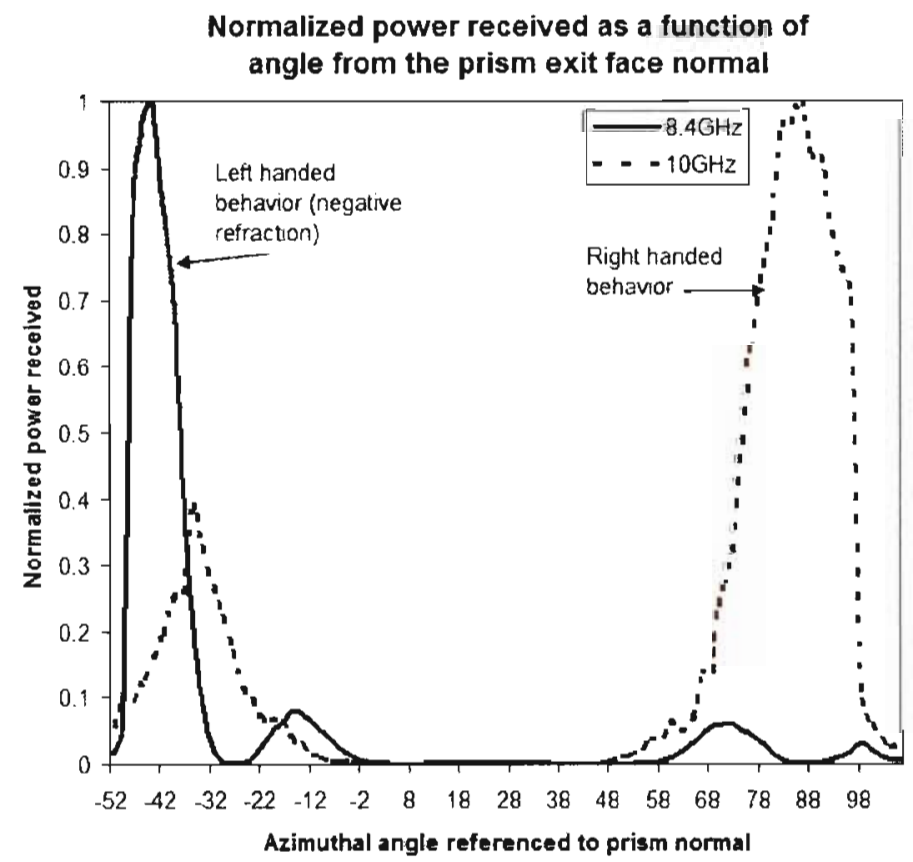

Figure 11.14 Normalized power received for two frequencies. Negative refraction is clearly visible at $8.4 \mathrm{GHz}$, while at $10 \mathrm{GHz}$, the refraction clearly exhibits standard right handed behavior. 


\subsubsection{FR-4 only metamaterial prism for test setup confirmation}

A metamaterial prism with identical dimensions to those given in Figure 11.11 is constructed out of un-clad FR-4 boards. Such a test of a homogeneous prism structure theoretically results in microwave beam refraction in a direction indicative of right-handed materials. Furthermore, as there are no metallic inclusions in this prism structure, the microwave response of the FR-4 only prism is not frequency dependent. Hence, the index of refraction should remain constant over the entire frequency range under test $(7 \mathrm{GHz}$ to $10 \mathrm{GHz})$. The constructed prism structure is visible in Figure 11.16.

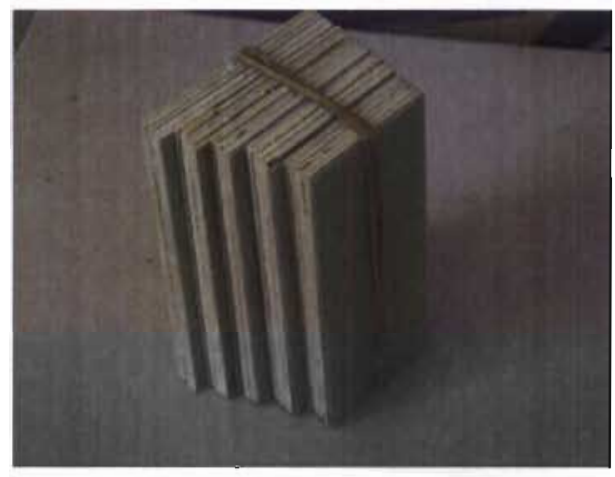

Figure 11.15 Fabricated FR-4 only metamaterial prism used to test the ability of the test setup to accurately measure the structure's index of refraction.

The prism normal direction is $26.56^{\circ}$ as indicated in Figure 11.16. This normal angle may be used in conjunction with Snell's law to obtain the material under test's index of refraction. As indicated by Snells law, given that the normal angle $\theta_{1}=26.56^{\circ}$ and the prism is placed in air $n_{1}=1.0$, the index of refraction, $n_{2}$ at a given frequency may be calculated as:

$$
n_{1} \sin \left(\theta_{1}\right)=n_{2}(\omega) \sin \left(\theta_{2}(\omega)\right)
$$

With knowledge that the nominal dielectric permittivity of FR-4 epoxy board is $\epsilon_{r}=4.4$, one 
can calculate the expected index of refraction to be:

$$
n_{2}=+\sqrt{\epsilon_{r} \mu_{r}}=+\sqrt{4.4 \cdot 1}=2.097
$$

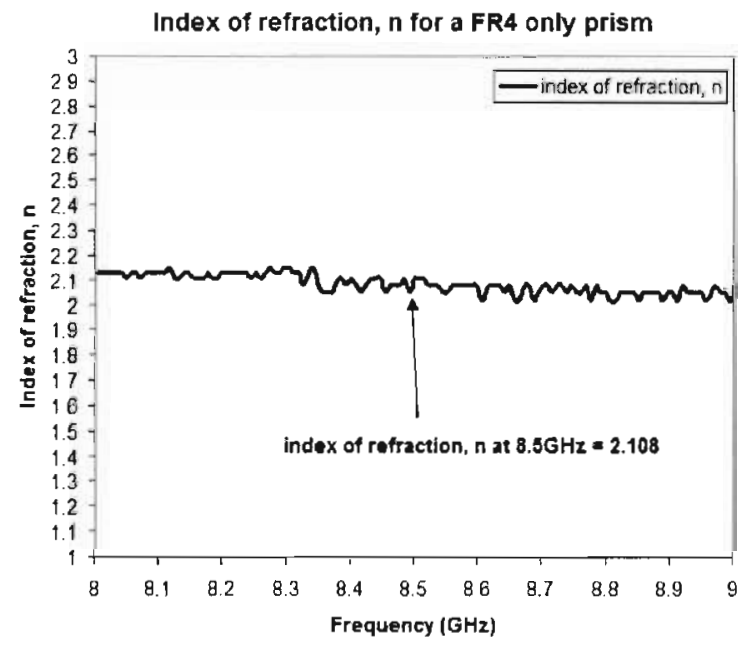

Figure 11.16 Index of refraction obtained from a FR-4 only prism.

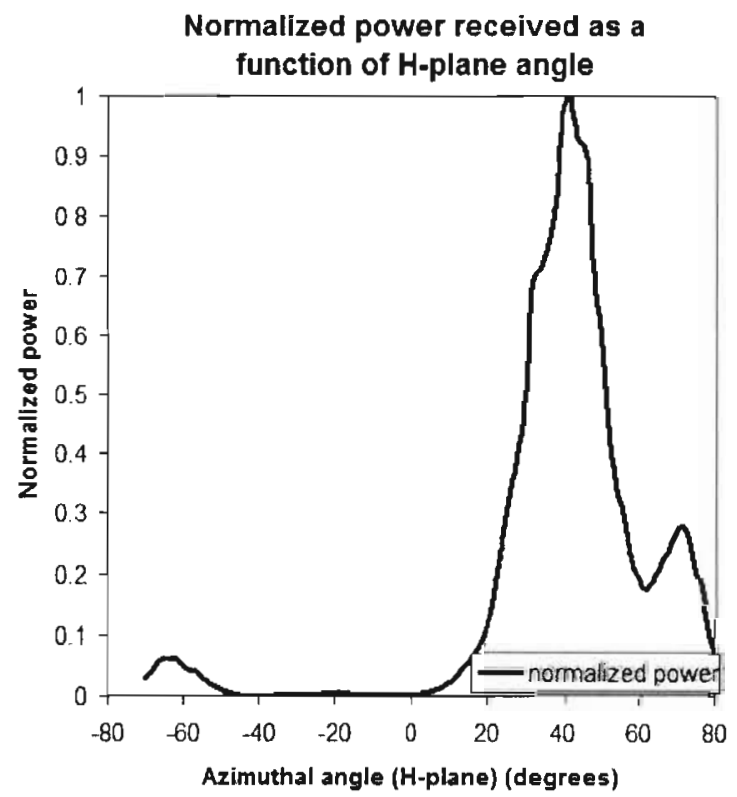

Figure 11.17 Normalized power received at $8.5 \mathrm{GHz}$ for the FR-4 only prism. 
The calculated index of refraction at $8.5 \mathrm{GHz}$ as indicated in Figure 11.16 is 2.108 and shows that there is a strong agreement between the theoretical index of refraction of the FR-4 only prism and the test results obtained from radiation pattern testing in the Anechoic chamber. The \% difference between the theoretical and measured index of refraction is $0.52 \%$. Furthermore, the non-frequency dependent nature of the FR4-only prism is visible in the index of refraction remaining constant over the test frequency range. The agreement between test data and theoretical values indicate that the test setup is indeed valid. Figure 11.17 indicates that maximum power was received by the HP receive waveguide horn at approximately $40^{\circ}$ from the prism normal. 


\section{Chapter 12}

\section{Application: Directivity and Power}

\section{Enhancement through sub-wavelength}

\section{apertures}

\subsection{Theory}

Several groups in the physics community have had recent success in enhancing power transmission through nano-scale holes in the optical domain. Due to leaky waves induced by periodically corrugated metallic screens surrounfing a nano-scale aperture, some physicists suspected constructive and destructive interference as a possible cause for power enhancement. However, A.A. Oliner reported in [24] that the phenomenon can be attributed to leaky wave plasmons excited by the corrugated screen surface. These are resonant excitations of the electron density on a surface. Note that metallic screens used in these experiments must be excited at a plama frequency in order to allow the presence of surface plasmons on the screen. However, metamaterials provide an alternative way of possibly enhancing directivity and power through sub-wavelength holes. Alù and Engheta [27] suggest that a metamaterial covering the sub-wavelength hole in a PEC screen also excites leaky waves at the cover interface. 
A single sub-wavelength hole in a PEC or absorbing screen would limit power transmission received broadside $\left(\phi=90^{\circ}\right)$. Additionally, diffraction at the aperture would cause an emanating spherical wave to radiate out the aperture. The addition of a metamaterial slab on the backside and/or front side of the aperture may have the effect of enhancing directivity and power transmission by exciting leaky modes. A heuristic ray diagram for the ideal operation of backside and front side metamamterial covers of a sub-wavelength aperture is shown below.

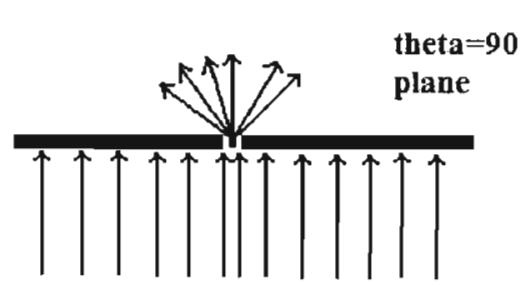

no slabs

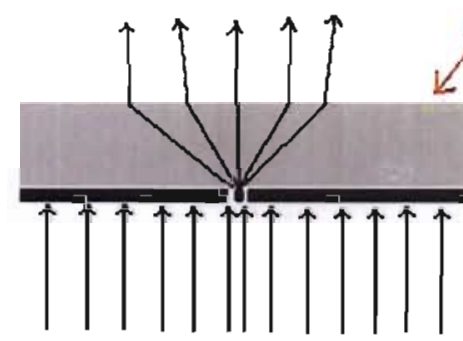

low $k$ slab on exit face

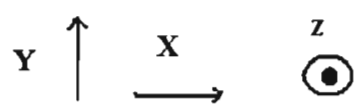

(อ)

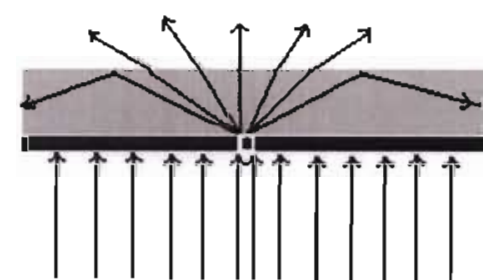

$k \gg k 0$ slab on exit

face

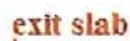

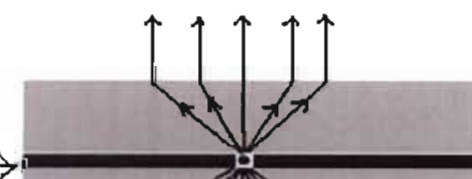

PEC

screen

entrance

slab

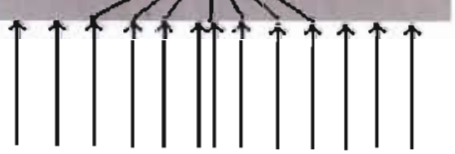

low $k$ slabs on both

faces

Figure 12.1 Ideal Ray diagrams showing the impact of diffraction due to a sub-wavelength aperture in 4 configurations: 1)No exit or entrance metamaterial slab,2)Exit only slab with $\left.k>>k_{0} .3\right)$ Exit only slab with $k<<k_{0}$ and 4)Entrance and exit slab with $k<<k_{0}$. 
Engheta in [27] emphasizes the use of slabs that have the constitutive parameter $k<<k_{0}$. Hence: $|k|=\omega|\sqrt{\mu \epsilon}|<<k_{0}=\omega \sqrt{\mu_{0} \epsilon_{0}}$. Clearly the product in the square root must be less than 1 to meet this criteria. Negative index metamaterials are frequency dependent. At certain frequencies they exhibit left-handed behavior and negative constitutive parameters $\mu, \epsilon$. However, they too may fail to meet the criteria if the product in the square root exceeds 1 . The notion of low $k$ or epsilon near zero metamaterials has been proposed. Epsilon near zero metamaterials (ENZ) have been coined as a term to describe a homogeneous artificial material whose permittivity over certain frequency ranges is close to zero. One can see that with epsilon close to zero, $k<<k_{0}$. The effect of double negative and ENZ materials as a means to increase directivity is simulated in HFSS.

\subsection{HFSS simulations}

\subsubsection{Ideal simulations}

In this case, a metamaterial slab with material parameters $\mu_{r}$ and $\epsilon_{r}$ directly specified within HFSS is used. The dielectric loss tangent, $\delta$ of the slab medium is set to zero in order to simulate a lossless medium. Various parameters are considered in the simulation setup. These include metamaterial slab permeability and permittivity, sub-wavelength aperture dimensions, and metamaterial slab thickness.

One must note that in order to reduce simulation time, the height of the setup in the $\mathrm{Z}$ direction is limited to $10 \mathrm{~mm}$. Periodicity in the vertical direction is mimicked by the periodic boundary conditions implemented by the full wave solver. However, with the presence of radiation boundaries for far field measurements, results are limited to radiation patterns in the azimuthal plane. Hence directivity is measured as a function of the E-plane cross-pole direction, $\phi$. On the other-hand, reducing the width of the setup ( $x$ direction) and increasing the height in the $z$ direction to be greater than the incident EM field $\lambda$, one can obtain measurements of the radiation pattern as a function of the co-pole direction $\theta$. Flaring in both dimensions increases simulation time for the full-wave solver but allows one to obtain E-plane patterns in the co-pole and cross-pole directions. 


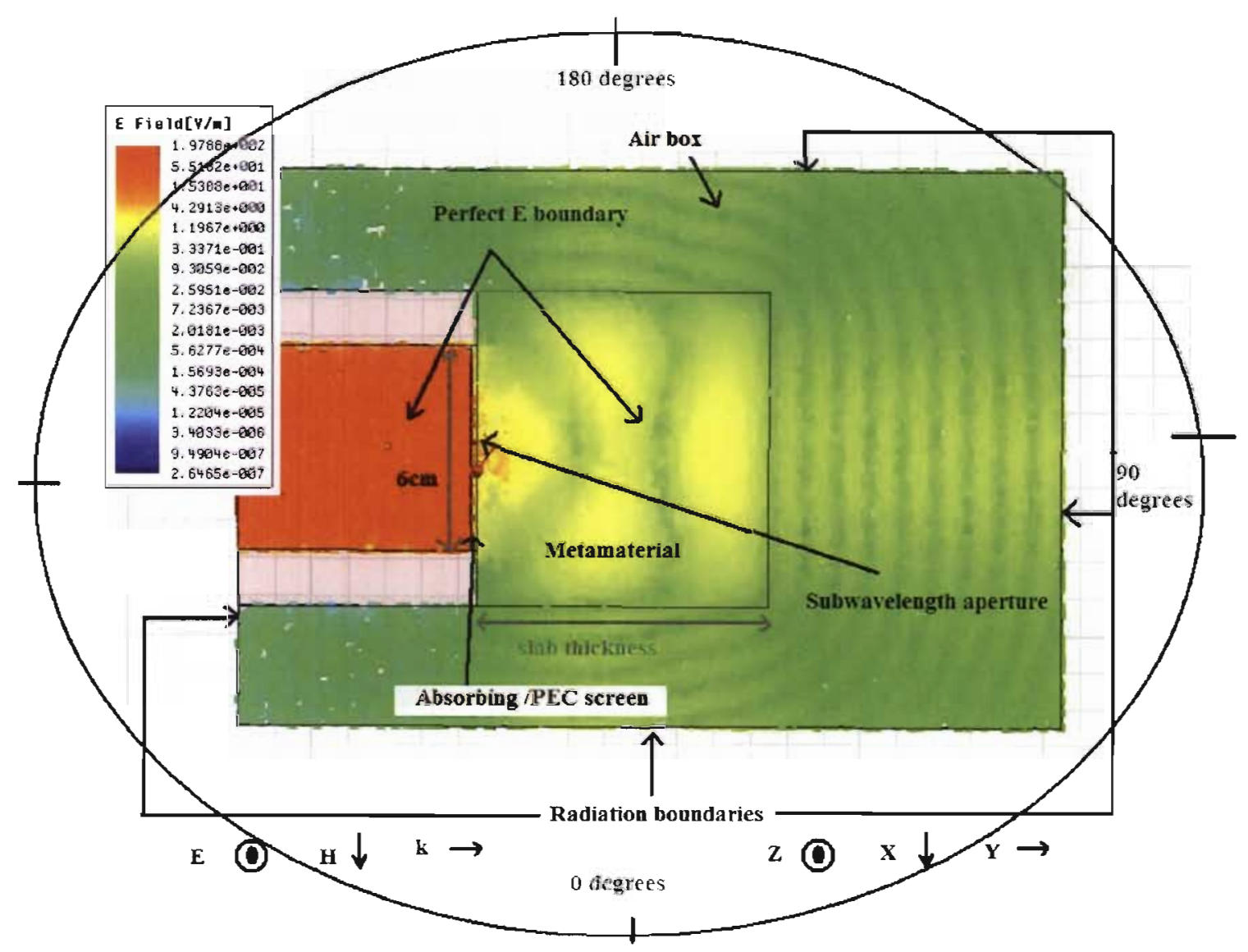

Figure 12.2 Top down view of simulation setup in HFSS. Parameters such as aperture size, exit slab thickness and air box size/depth may be varied with the use of variables. Default settings are aperture size $=5 \mathrm{~mm} \times 5 \mathrm{~mm}$. Slab thickness $=54 \mathrm{~mm}$ and air box depth in the $z$ direction $=10 \mathrm{~mm}$. A circle has been drawn around the setup to resemble the far field sphere set by HFSS. The goal is to maximize directivity and gain in the $\phi=90^{\circ}$ direction. 


\section{Effect of $\epsilon_{r}$ and $\mu_{r}$ on a slab placed on the aperture exit face}

The permittivity and the permeability of the homogeneous slab are changed directly within HFSS. For each variation, the peak directivity and more importantly the directivity at $\theta=90^{\circ}$ and $\phi=90^{\circ}$, is recorded (see Table 12.1). This is the direction directly normal to the sub-wavelength aperture and metamaterial slab.

\begin{tabular}{||c|c|c|c||}
\hline$\epsilon_{r}$ & $\mu_{r}$ & Peak directivity & directivity @ $\theta=90^{0}, \phi=90^{\circ}$ \\
\hline 4.4 & 1 & 1.7256 & 0.0017 \\
\hline 3.3 & 1 & 2.8684 & 1.4808 \\
\hline 2.2 & 1 & 2.1779 & 1.3031 \\
\hline 1 (no slab) & 1 (no slab) & 4.9481 & 2.4928 \\
\hline 0.5 & 1 & 4.373 & 4.3307 \\
\hline 0.1 & 1 & 10.9017 & 10.9017 \\
\hline 0.001 & 1 & 10.123 & 9.3158 \\
\hline 0.001 & 10 & 15.033 & 10.5494 \\
\hline 0.001 & 0.001 & 14.365 & 13.2161 \\
\hline 1 & 0.001 & 14.369 & 13.433 \\
\hline-0.001 & -0.001 & 13.403 & 11.6013 \\
\hline-0.01 & -0.01 & 10.8581 & 7.4692 \\
\hline-0.1 & -0.1 & 12.3045 & 12.3045 \\
\hline-1 & -1 & 2.8321 & 2.8321 \\
\hline-2 & -1 & 3.5945 & 1.7479 \\
\hline-3 & -1 & 4.6824 & 1.4559 \\
\hline-4 & -1 & 5.5826 & 2.7201 \\
\hline
\end{tabular}

Table 12.1 Table shows relationship between exit face metamaterial $\mu_{r}, \epsilon_{r}$ and directivity. As permeability and permittivity is reduced, $k<0$ and the directivity is enhanced.

As to be expected low $k$ dielectrics produce improved directivity in the far field. Negative index dielectrics however, do not perform the same function. Infact, viewing a radiation field overlay in HFSS allows one to realize that a focal point is in fact being generated in the near field. This focal point may be considered a point source similar to that produced by a sub-wavelength aperture. Hence, a diverging spherical wave is emitted in the direction of propagation from this focal point and the beam detected in the far-field is not directive as compared to the low $|k|$ material. 


\section{Effect of exit slab thickness on directivity and gain}

The thickness of the exit face metamaterial slab is an important consideration. Ideal HFSS simulations where lossless isotropic materials are used show that increasing the slab thickness increases directivity. However, this increase is not linear and does not increase infinitely as seen in Figure 12.3. A larger slab thickness means that the spherical wave generated by diffraction in the aperture becomes a plane wave within the slab itself. Internal reflection may occur on the side walls of the slab limiting side leakages. At the exit slab air interface, the plane wave is less partial to refraction away from the normal. Hence one sees a more directive, non-diverging wave emanating from the exit metamaterial slab. Gain measurements show that the PEC abosrbing screen prevents the majority of the radiation from passing through. Hence, gain measurements show low power transmission values ranging from -32 to $-48 \mathrm{~dB}$. A peak in gain is visible at higher thicknesses from 50 to $65 \mathrm{~mm}$ but drops off elsewhere. The thickness of the slab and the simulation parameters are such that the E field is especially concentrated within the slab. It must be noted that in practice, thicker slabs are susceptible to higher dielectric losses which may offset any enhancement benefits from adding an exit slab behind the sub-wavelength aperture. There is no relation available to properly select a slab thickness. Simulations must be run for a certain design beforehand. 


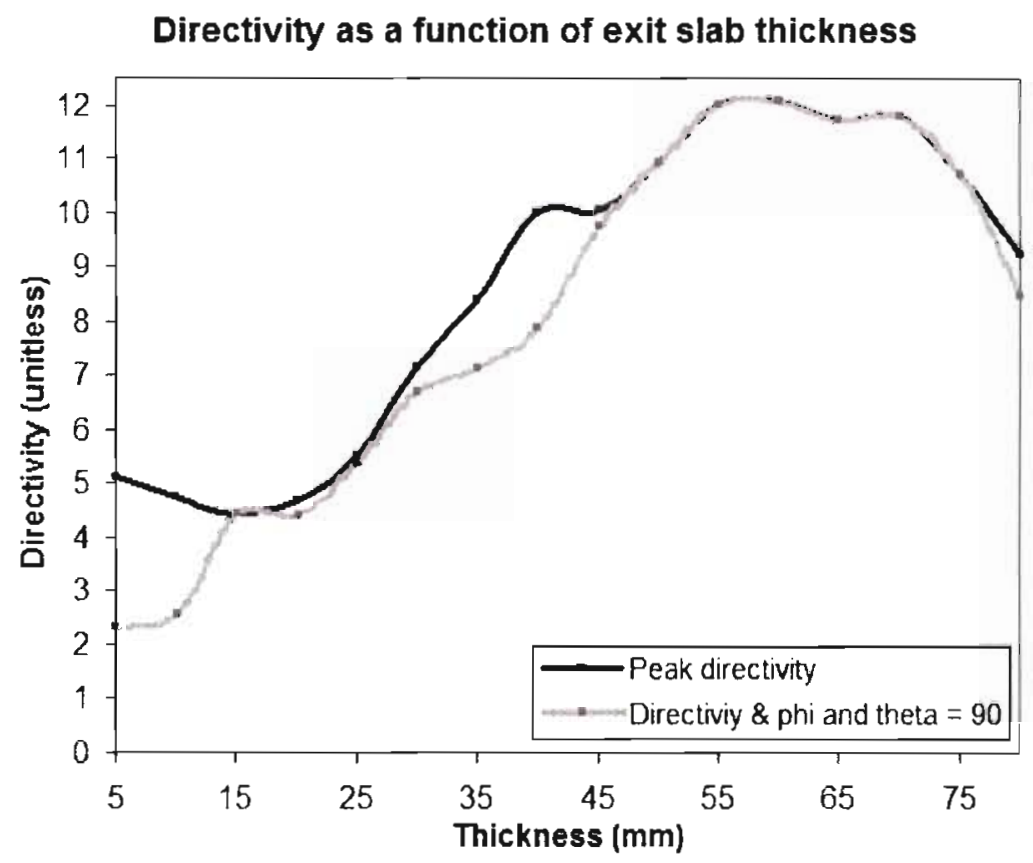

Figure 12.3 Block diagram for proposed testing of DNG metamaterials in the Anechoic Chamber.

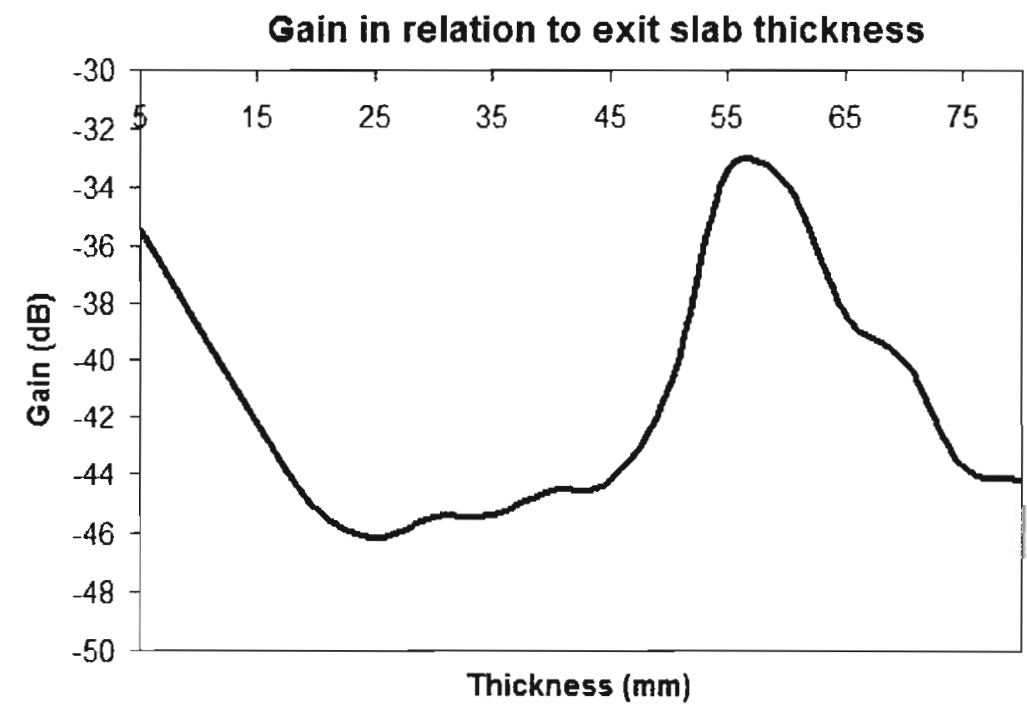

Figure 12.4 Sideview of testsetup structure. 


\section{Effect of aperture size on directivity and gain}

Heurestically, increasing aperture size increases gain, as less of the illuminating plane wave is absorbed by the PEC absorbing screen. However, increasing aperture size infinitely will have show no added improvement in directivity. At aperture sizes much greater than the incident wave's $\lambda$, diffraction at the screen interface is minimal. Hence while a zero index metamaterial would improve directivity, the increase is not linear with aperture size. An analysis of aperture size versus broadside directivity and gain is studied via ideal HFSS simulations.

The results in Table 12.2 clearly confirm the heuristic hypotheses stated above. Gain increases with aperture; especially above the illuminating EM wave's wavelength. However, gain flattened out for larger widths. This is due to the fact that the during simulation testing, the aperture width was increased at the expense of the PEC absorbing screen width. Assuming the illuminating beam width is limited to a width less than or equal to the PEC screen width (a parallel TEM mode waveguide configuration is used in the HFSS simulations), then the aperture width too is limited to the PEC screen width. As gain relates to a radiating devices efficiency and inherent losses, it is expected that a horizontal asymptote on gain measurements exists when losses are negligible.

\begin{tabular}{||c|c|c||}
\hline Aperture width $(\mathrm{mm})$ & directivity @ $\theta=90^{\circ}, \phi=90^{0}$ & Gain $(\mathrm{dB})$ at $\phi$ and $\theta=$ 90degrees \\
\hline 0.5 & 11.4758 & -50.1682 \\
\hline 1 & 14.4472 & -50.6924 \\
\hline 5 & 11.645 & -40.476 \\
\hline 10 & 11.9633 & -12.618 \\
\hline 20 & 11.9691 & 5.9343 \\
\hline 30 & 12.004 & 9.2214 \\
\hline 40 & 12.03 & 9.6897 \\
\hline 50 & 12.198 & 10.3 \\
\hline 60 & 12.2043 & 10.5 \\
\hline 70 & 12.2592 & 10.5742 \\
\hline 80 & 12.2978 & 10.6176 \\
\hline 90 & 12.2568 & 10.5333 \\
\hline 100 & 11.793 & 9.9318 \\
\hline
\end{tabular}

Table 12.2 Table shows relationship between aperture width, directivity and Gain. HFSS simulation results at $8.5 \mathrm{GHz}$ are obtained using a homogeneous ideal slab with zero loss tangent $\delta, \epsilon_{r}=0.1$ and $\mu_{r}=0.1$. The slab thickness was held constant at $54 \mathrm{~mm}$ 


\subsubsection{Metallic inclusions embedded in exit face dielectric slab}

The results for previous simulations presented in this chapter involved a material slab generated in HFSS that had bulk parameters $\epsilon_{r}$ and $\mu_{r}$ specified directly in the software. Furthermore, dielectric loss tangents of the material acting as an exit face slab behind the aperture were set to zero. Such a setup confirms the theory behind the use of epsilon near zero metamaterials to enhance directivity. However, when considering practical implementations it is important to consider how the metamaterial should be fabricated and with what inclusions. Dielectric losses must also be considered.

\section{Thin wire rod structure}

A thin wire structure is considered as a means to achieve a permittivity close to zero hence reducing $k$. The rods in Figure 12.5 have a diameter of $0.2 \mathrm{~mm}$ and are spaced apart by $5 \mathrm{~mm}$ in the $\mathrm{X}$ and $\mathrm{Y}$ directions. A PEC/PMC waveguide simulation with the rods embedded in FR4 substrate in HFSS indicates that resonance in the scattering parameters occurs at approximately $9 \mathrm{GHz}$. This resonance frequency serves as the point at which the dielectric material acts like a plasma medium and where evanescent modes no longer dominate transmission. The inclusions are used in the sub wavelength aperture test simulation setup described previously. Results in Figure 12.6 show that a two fold enhancement in directivity is achieved compared to a setup without the presence of a metamaterial on the exit slab. However, at $9.05 \mathrm{GHz}$, the peak in beam directivity in the $\mathrm{H}$-plane is seen at $\phi=70^{\circ}$ and not the desired $\phi=90^{\circ}$. Refraction is occurring within the metamaterial medium and at the metamaterial/air interface. 


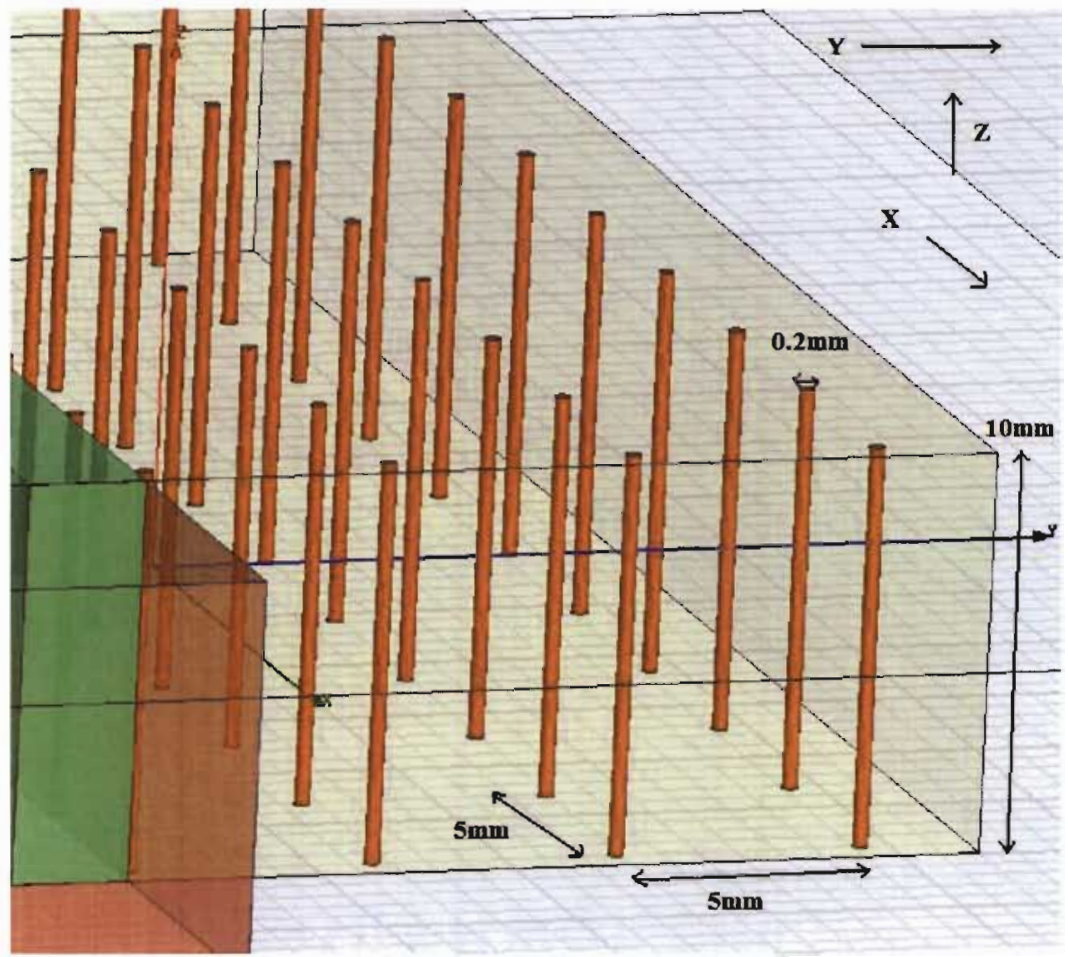

Figure 12.5 Thin wire rods embedded in exit aperture slab.

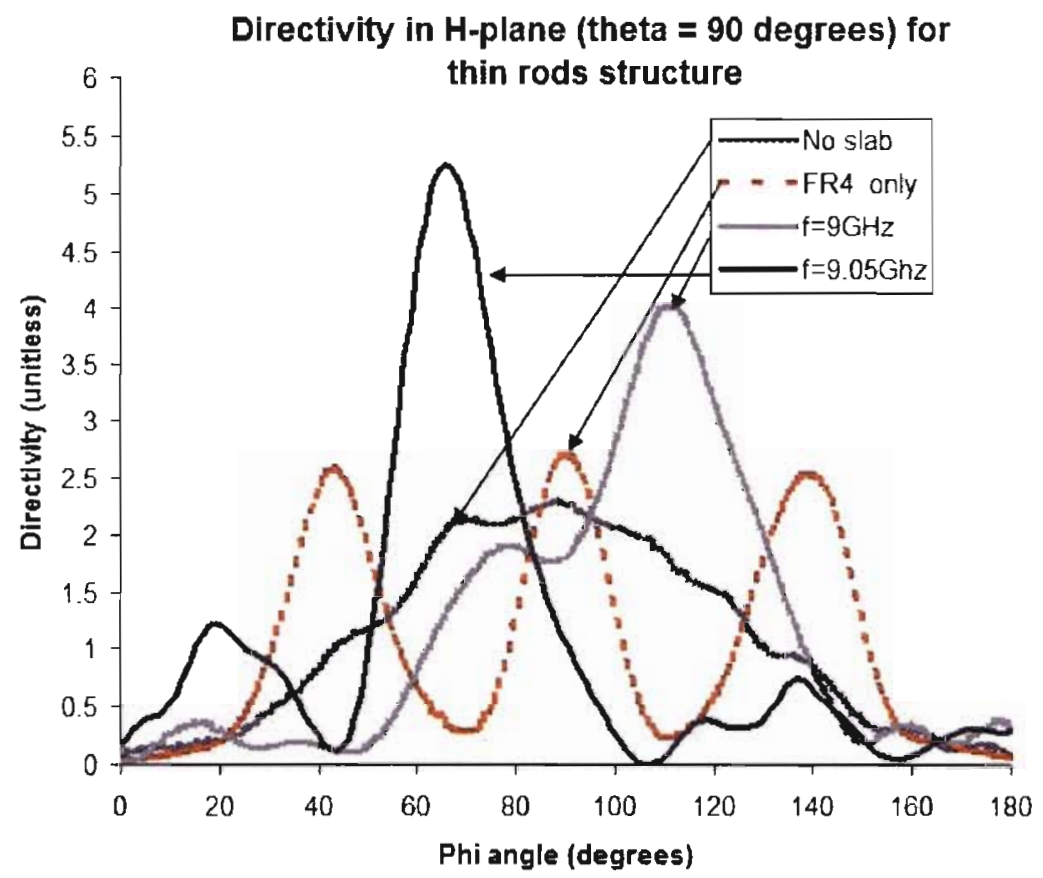

Figure 12.6 Beam directivity in the azimuthal plane from a sub-wavelength aperture covered with a slab consisting of thin rod structures. Slab thickness is $22 \mathrm{~mm}$. 


\section{S-shaped SRR structures}

Of particular interest are the double negative index metamaterial structures designed in this project. The S-shaped SRR structure designed, analyzed and simulated previously provides a means by which to investigate the possibility of beam enhancement. The inclusion dimensions are unchanged from the simulations for the transmission slab developed previously. As with the thin wire simulations, the inclusions are embedded in a FR-4 slab placed directly behind a sub-wavelength aperture. As $\mu$ and $\epsilon$ are frequency dependent, it is also expected that any directivity enhancement due to the value of $|k|$ being much less than one is also frequency dependent. Results show that peak directivity at $8 \mathrm{GHz}$ (found to produce the best results of all tested frequencies) is $20 \%$ less directive than the rods structure at $9.05 \mathrm{GHz}$. However unlike the thin rods structure, peak transmission occurs at $\phi=90^{\circ}$ which is desired.

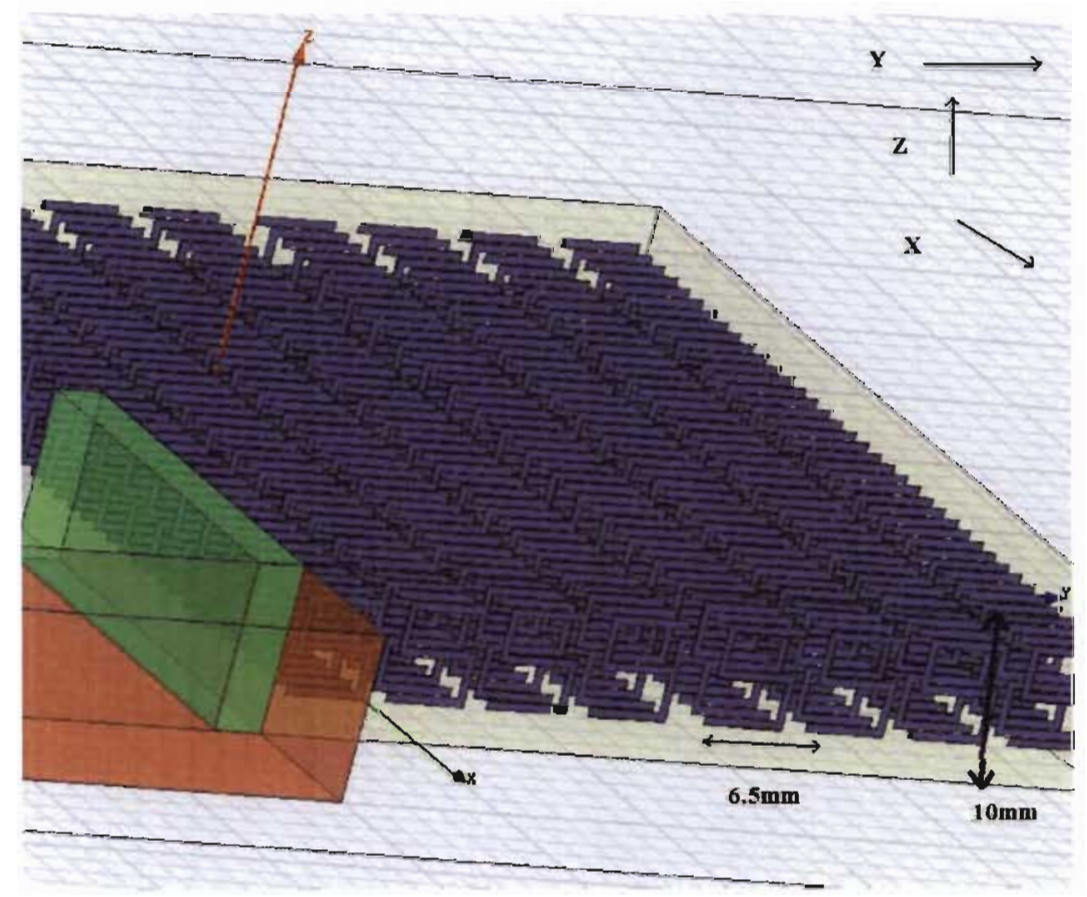

Figure 12.7 S-shaped split ring resonators embedded in exit aperture slab.

Directivity is not a complete indicator of the increase in power transmitted at a certain angle in the H-plane. Gain measurements take into account losses in the system. Due to the resonance in the transmission of these frequency dependent metamaterial structures, a peak in directivity may 


\section{Directivity in the H-plane (theta=90degrees) for a S-shaped SRR}

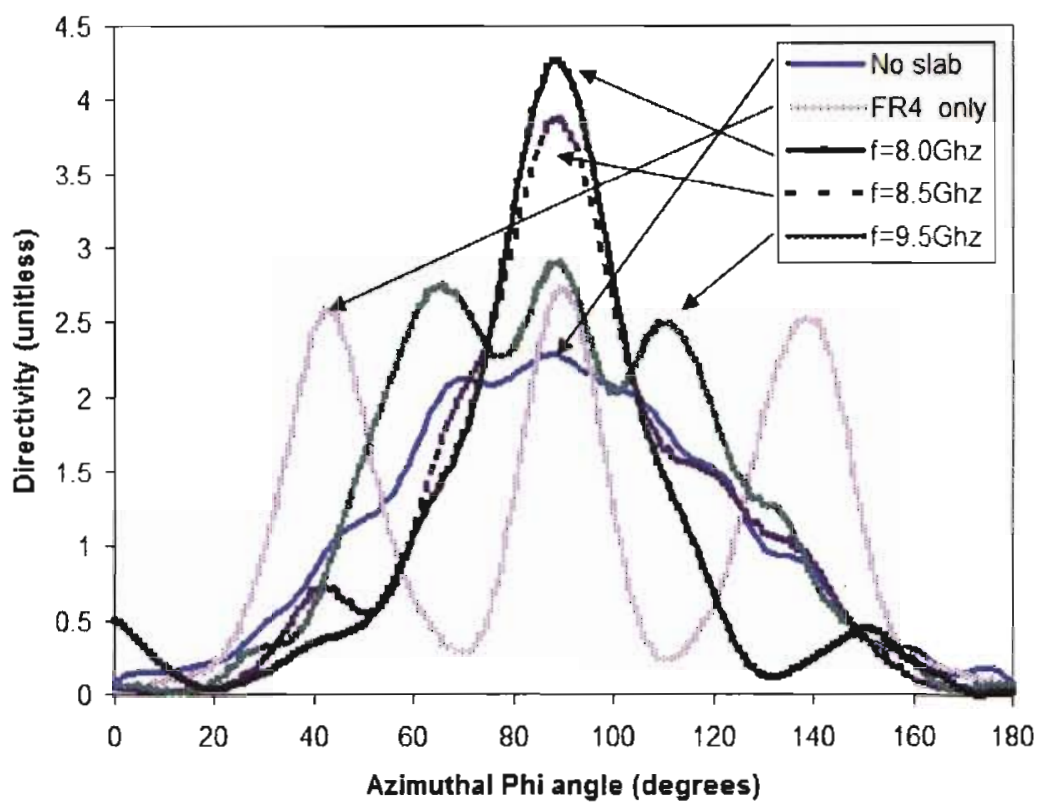

Figure 12.8 Beam directivity in the azimuthal plane from a sub-wavelength aperture covered with a slab consisting of S-shaped resonators. Slab thickness is $54 \mathrm{~mm}$.

not correspond to the slab's match to free space. As $k$ approaches zero, if the slab's transmission S-parameter indicated by $\left|S_{21}\right|$ does not approach $0 \mathrm{~dB}$, losses in the slab are inevitable. In this case, measured gain as a function of azimuthal angle in the $\mathrm{H}$-plane is less than OdB. Figure 12.9 shows that for the S-SRR structure, losses in the region of negative index of refraction and high directivity (Figure 12.8) losses occur in the metamaterial slab. Gain enhancement is measured by taking Gain simulations from HFSS with no exit slab and with an exit slab. The ratio between simulated gain with no slab and simulated gain with the S-SRR slab defines the enhancement factor. The losses in the S-SRR slab are due to several reasons. The slab dielectric (FR-4) has a loss tangent $\delta=0.02$. At $8.5 \mathrm{GHz}$, this may be reduce peak transmission by $7 \mathrm{~dB}$ or more as seen in Figure 7.13. Furthermore, the slab thickness used in simulations for the S-SRR structure is $54 \mathrm{~mm}$. Thicker structures exhibit greater loss which prevent gain enhancement $\geq 0 d B$. Resonance in transmission also prevents a gain enhancement from being constant over a wide frequency range. 


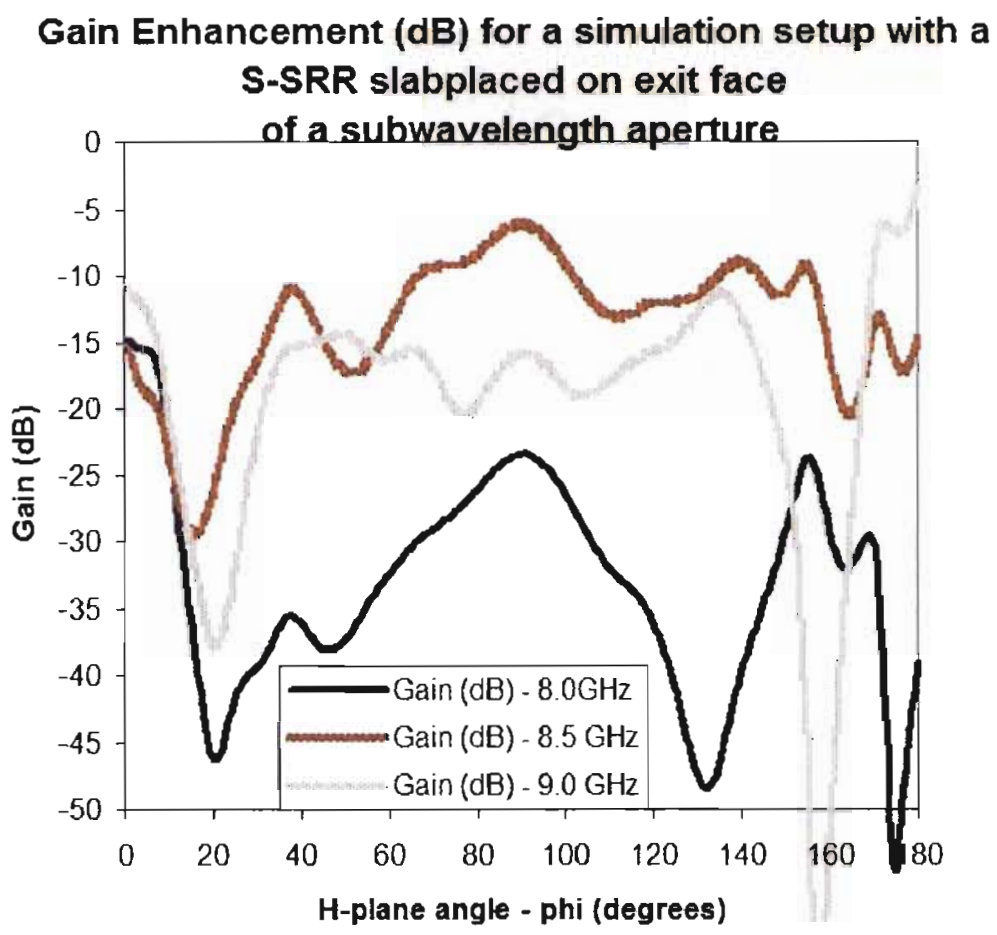

Figure 12.9 Gain Enhancement for a S-SRR slab placed on the exit face of a subwavelength aperture in comparison to the case that no slab is present.

\section{Grid lattice structure}

A grid lattice structure was used by Enoch et al [26] in 2002 in an experiment to show directivity enhancements when a monopole antenna is embedded within the metamaterial itself. This design is extended to the case of placing a grid mesh structure of wire strips on the exit face of a subwavelength aperture. The response as shown in Figure 12.11 is similar to that of thin wire rods or strips. The unit cell has been designed for a plasma resonance at approximately $8 \mathrm{GHz}$. 


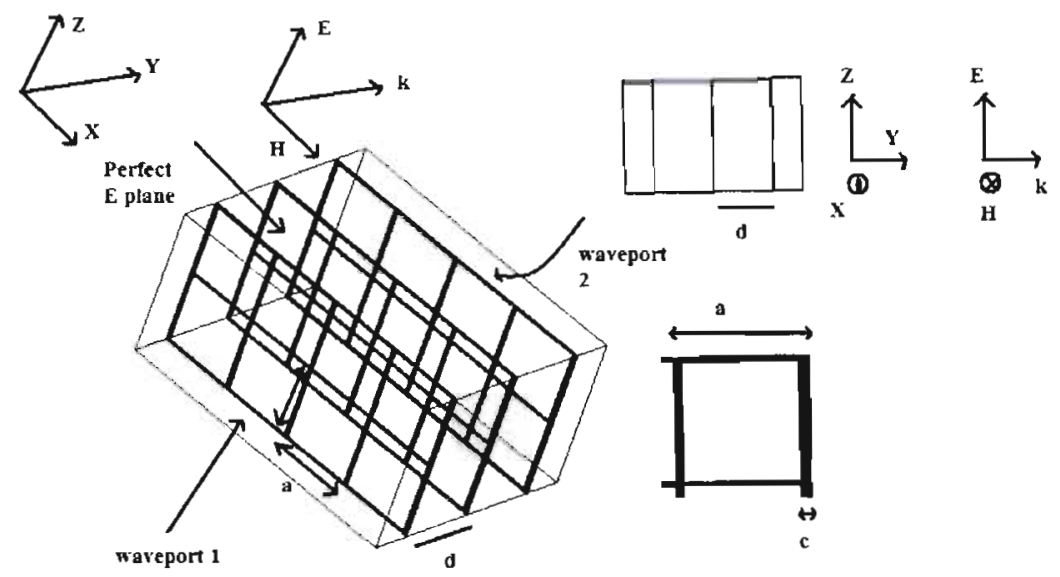

Figure 12.10 Grid mesh unit cell designed for a plasma frequency at approximately $8 \mathrm{GHz}$. Lattice paramater $\mathrm{a}=5.0 \mathrm{~mm}$ and copper strip width $\mathrm{c}=0.2 \mathrm{~mm}$. Spacing $=6 \mathrm{~mm}$.

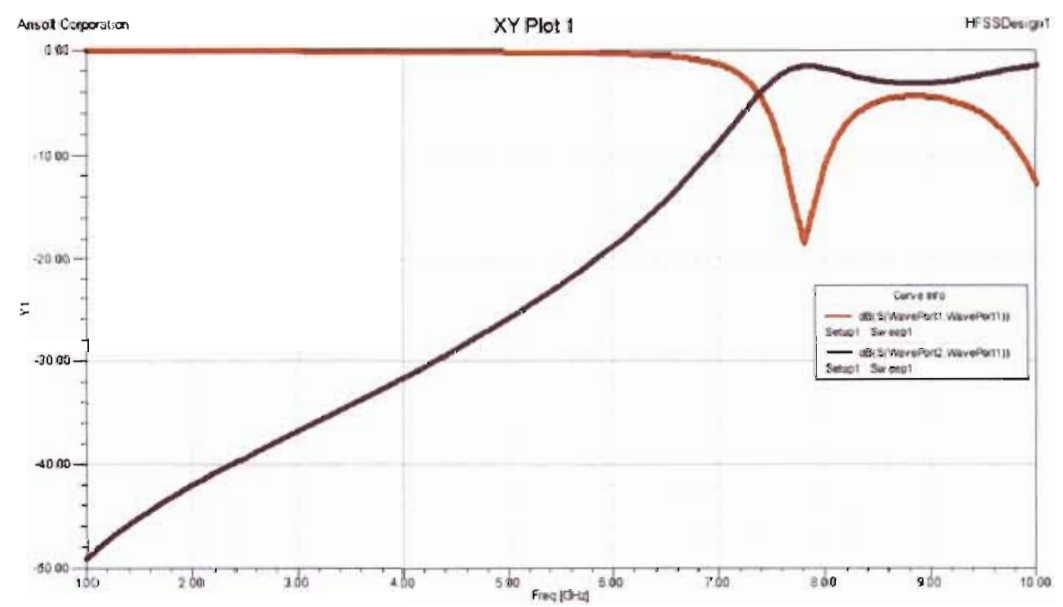

Figure 12.11 Transmission simulation results for grid mesh structure shown in Figure 12.12. A plasma resonance is seen at approximately $8 \mathrm{GHz}$. In this region it is expected that $\epsilon_{r}$ and $n$ approaches zero and hence $k<<1$. 


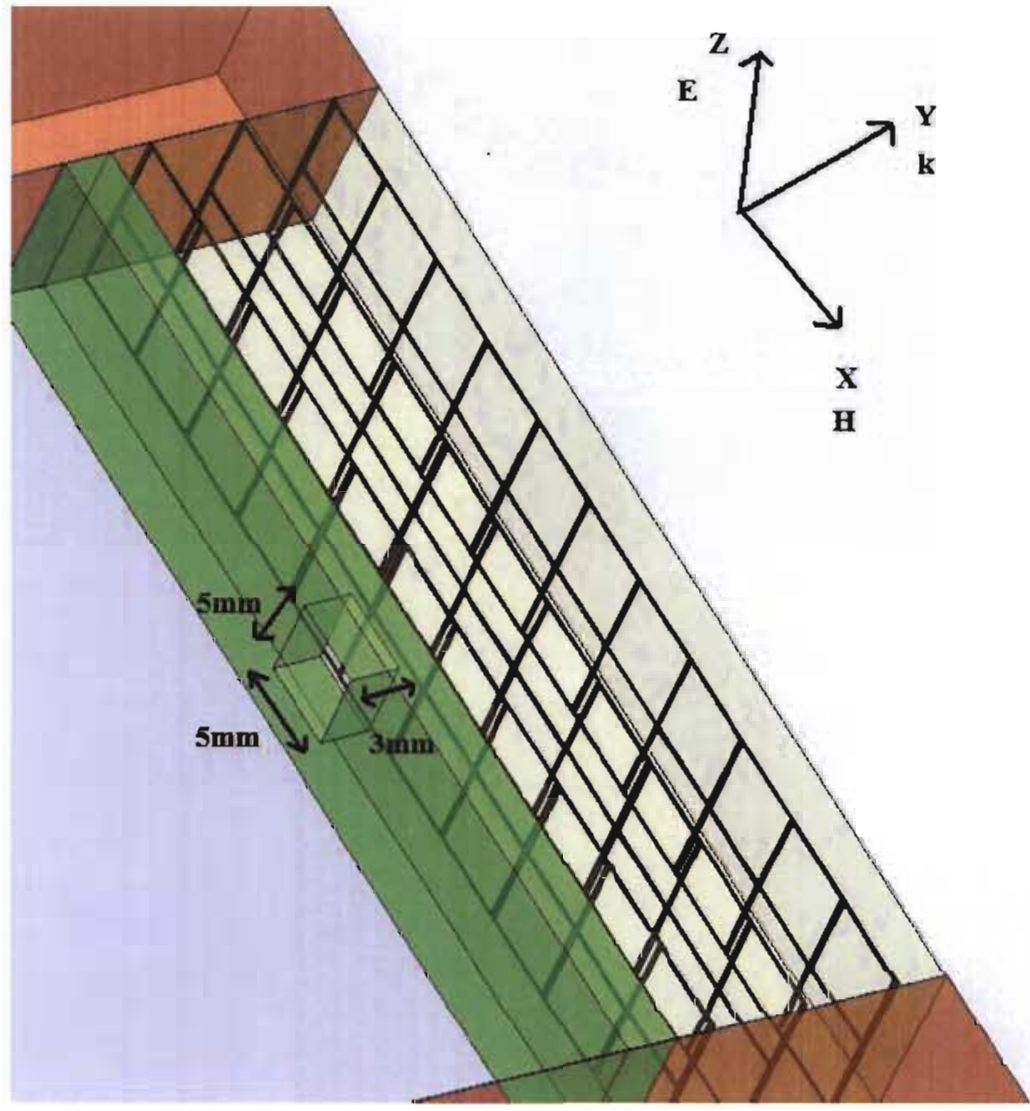

Figure 12.12 Grid mesh structures embedded on the exit face of the sub-wavelength aperture in HFSS. There are 3 lattice elements in the $Y$ direction leading to a slab thickness of $18 \mathrm{~mm}$. 
Far field simulations in HFSS are conducted in Ansoft HFSS. Results show that directivity is enhanced by a factor of two for input frequencies in the 8 to $9 \mathrm{GHz}$ range in comparison to the case when no exit slab is placed on the sub-wavelength aperture exit face. These results are summarized in the plots of Figure 12.13. Refraction away from the slab and aperture normal ( $\phi=90$ degrees in HFSS far field simulations) is visible indicating the structures are not perfect directivity enhancers and that refraction occurs within the metamaterial medium. The structures may need tuning to obtain peak directivity and gain at exactly $90^{\circ}$. This entails modifying the rod radius and lattice spacings as well as the slab thickness.

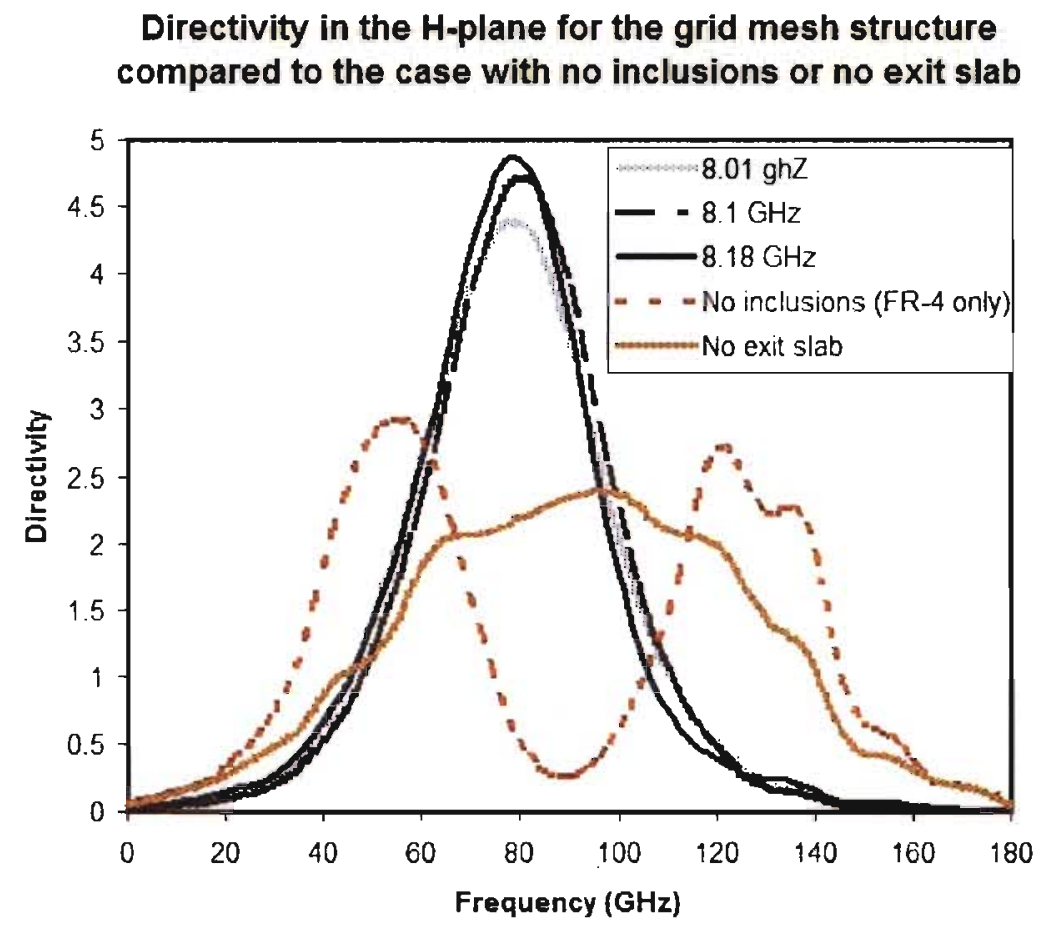

Figure 12.13 Directivity when Grid mesh is placed on the exit face of the aperture. Far field plots are presented for several frequencies near the plasma resonance of the grid mesh structure.

Gain enhancement simulations show that $\mathrm{A}$ gain enhancement of $10 \mathrm{~dB}$ is seen at azimuthal angles of high directivity in the case of a $8.04 \mathrm{GHz}$ source. The improved gain enhancement is primarily due to operating at the wire structure's plasma frequency where transmission is superior to the S-SRR structure (Figure 12.12). Furthermore, slab thickness used in this simulation is only 
$17 \mathrm{~mm}$ while the S-SRR slab was simulated with a thickness of $54 \mathrm{~mm}$ in an effort to improve directivity. Note that Figure 12.3 indicates that directivity increases as a function of exit slab thickness. Due to the thinner slab, dielectric losses have less of an impact and the focusing of energy in a certain direction supersedes such losses. Notice that at $8.18 \mathrm{GHz}$, a drop in gain is seen. Like the S-SRR structure, this may be attributed to a small resonance in $\left|S_{21}\right|$ as seen in Figure 12.11 .

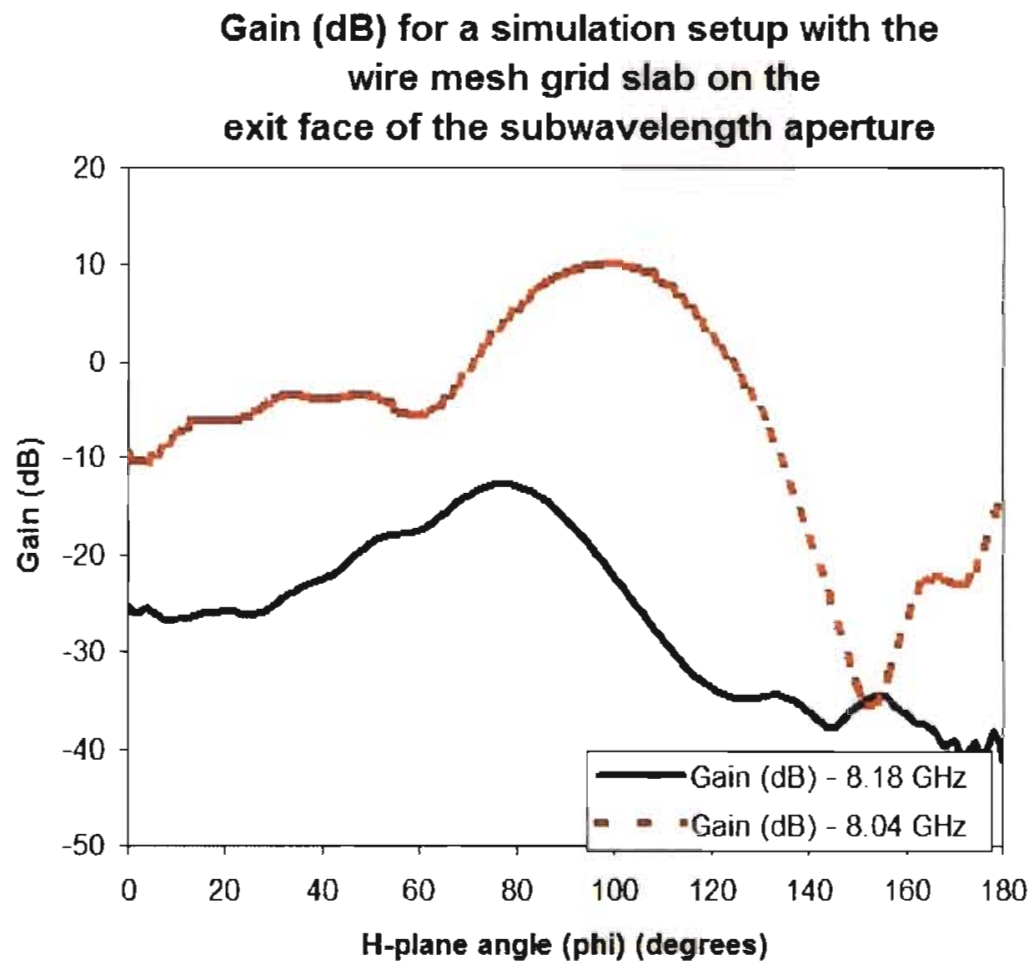

Figure 12.14 Gain Enhancement for a S-SRR slab placed on the exit face of a subwavelength aperture in comparison to the case that no slab is present. 


\section{Chapter 13}

\section{Conclusions}

In this project, the concept of periodic structures embedded in a dielectric substrate has been shown via theoretical analysis, simulation and experimentation to possess permeability and permittivity with negative real components. HFSS simulations show that a theoretical material exhibiting lefthand properties has a phase velocity anti-parallel to the group velocity and Poynting vector.

Copper inclusions introduced by research groups around the world have been theoretically studied. The divergence or vertical asymptote in permeability was derived for the split ring resonator (SRR) structure. Simulations and test data proved that the derivations are approximate and that

it is necessary to perform transmission tests. A similar method was used to obtain the frequency dependent permeability relation for the S-shaped ring resonator. Thin rod structures in an array do not exhibit evanescent wave propagation at the plasma frequency. This has been confirmed via transmission simulations in HFSS and parameter extraction. The modified Nicolson-Ross-Weir extraction method [10] is shown to predict resonances in the reflection and transmission coefficients of the slab structure.

HFSS was used to perform parametric analysis on the quadrilateral split ring resonator and $\mathbf{S}$ shaped split ring resonator structure. The height of both structures was varied while keeping other paramaters such as the copper trace width, gap width and lattice spacing constant. Designs were optimized for a resonant frequency of $8.5 \mathrm{GHz}$. The $\mathrm{S}$-shaped resonator designed to exhibit left-handed properties was shown to have negative refractive properties using a prism simulation. Negative re- 
fraction was visible from 7.8 to $9.5 \mathrm{GHz}$. A custom test configuration designed for transmission and refraction testing was setup in the Cal Poly Anechoic Chamber. Transmission slab structures with quadrilateral SRR/CLS and S-SRR inclusions were built. The S-SRR structure transmission characteristics was shown to match closely with simulations. The resonance in transmission was shifted by several hundred $\mathrm{MHz}$ but magnitude values for $S_{21} d B$ were within $3 \mathrm{~dB}$ at the transmission peak for both sets of data. Parameter extraction using the modified Nicolson Ross Weir equations predicted regions of left-handed behavior and resonances in permeability and permittivity. However, the method is shown to be sensitive to phase noise which is reflected in the anomalous readings found in the extraction results. A prism structure has been fabricated and tested in the Anechoic Chamber. The index of refraction was found to be negative in the frequency range of 8 to $9 \mathrm{GHz}$.

An extension of the metallic inclusions presented in this project has been made to the problem of increasing directivity and gain through a sub-wavelength aperture. A structure of thin-wire rods was shown to influence directivity but at the expense of refraction away from the angle of interest. The s-shaped resonators and a grid mesh structure both were shown to improve directivity by a factor of 2 over a narrow band of $\mathrm{X}$-band frequencies. 


\section{Appendix A}

\section{Matlab Code}

Matlab code used to implement parameter extraction equations and plot permeability functions for the circular and $\mathrm{S}$-shaped split ring resonators is included in this section.

\section{A.1 Theoretical simulation of circular split ring resonator (SRR)}

Listing from source file SRR.m

\%SRR.m by Neil Patel

$\%$ Calculates resonance frequencues for vacuum and an other specified

\%dielectric for a circular SRR structure. Plots the permeability relation

\%based on chosen SRR parameters.

\%SRR parameters

$\mathrm{c}=1 \mathrm{E}-3 ; \%$ copper width

$\mathrm{d}=1.0 \mathrm{E}-4 ; \%$ gap width

$\mathrm{r}=2.0 \mathrm{E}-3 ; \%$ inner radius

$\mathrm{l}=2.0 \mathrm{E}-3 ; \%$ distance between rings in $\mathrm{H}$-plane

$\mathrm{a}=1.0 \mathrm{E}-2 ; \%$ distance between adjacent rings/unit cell width

$\mathrm{c0}=3 \mathrm{e} 8 ; \%$ freespace speed of light

\% free space constants

epsilon_0 $0=8.85 \mathrm{E}-12$

mu_0 $=1.257 \mathrm{E}-6$;

epsilon_vacuum $=1$;

epsilon_dielectric $=2.2 ; \%$ modify if different dielectric used.

\%calculate resonance frequency of SRR in a vacuum - permittivity equals 1 
\%omega_vacuum $=\operatorname{sqrt}\left(\left(3^{*} \mathrm{~d}^{*} \mathrm{c} 0^{\wedge} 2\right) /\left(\mathrm{pi}^{\wedge} 2 * \mathrm{r}^{\wedge} 3\right)\right)$;

omega_vacuum $=\operatorname{sqrt}\left(\left(3 * 1 * \mathrm{c} 0^{\wedge} 2\right) /\left(\mathrm{pi} * \log ((2 * \mathrm{c}) / \mathrm{d}) * \mathrm{r}^{\wedge} 3\right)\right)$;

freq_vacuum $=$ omega_vacuum $/\left(2^{*}\right.$ pi);

\%calculate resonance frequency of SRR on dielectric material

$\%$ (Duroid eps $=2.2$ or FR4 eps $=4.4$ )

omega_diel $=\operatorname{sqrt}\left(\left(3 * 1 * \mathrm{c}^{\wedge} 2\right) /\left(\mathrm{pi} *\right.\right.$ epsilon_dielectric $\left.\left.* \mathrm{r}^{\wedge} 3 * \log ((2 * \mathrm{c}) / \mathrm{d})\right)\right)$;

freq_diel $=$ omega_diel $/(2 * \mathrm{pi})$;

$\%$ fill matrix with frequency points

step_size $=0.01 \mathrm{E} 9$;

for $\mathrm{a}=1: 2000$

freq_matrix $(a, 1)=\left((0)+a^{*}\right.$ step_size $)$;

end

\%evaluate permeability relation for each frequency point.

omega_matrix $=$ freq_matrix.$*(2 * \mathrm{pi})$;

test $=\left(\left(3 * 1 *\left(\mathrm{c} 0^{\wedge} 2\right)\right) . /\left(\mathrm{pi} *\left(\right.\right.\right.$ omega_matrix .^2).$\left.\left.^{*} \log (((2 * \mathrm{c}) / \mathrm{d})) * \mathrm{r}^{\wedge} 3\right)\right)$;

mu_eff_num $=\left(p i * r^{\wedge} 2\right) / a^{\wedge} 2$;

mu_eff_den $=1+\left((2 * 1 * 200 * \mathrm{i}) . /\left(\right.\right.$ omega_matrix $\left.\left.*{ }^{*} \mathrm{r} * \mathrm{mu}_{-} 0\right)\right)-((3 * \mathrm{l} *$ $\left.\left(\mathrm{c} 0^{\wedge} 2\right)\right) . /\left(\mathrm{pi} . *\left(\right.\right.$ omega_matrix .^2) $\left.\left.*^{*} \log (((2 * \mathrm{c}) / \mathrm{d})) * \mathrm{r}^{\wedge} 3\right)\right)$;

mu_ratio $=$ mu_eff_num ./ mu_eff_den;

mu_eff $=1-10000000000 *$ (mu_eff_num ./ mu_eff_den);

mu_eff_real $=$ real $\left(m u \_e f f\right)$;

mu_eff_imag $=\operatorname{abs}($ imag $($ mu_eff $))$;

figure(1);

$\%$ Data plots

plot(freq_matrix ./ 1E9, mu_eff_real);

title('Real part of theoretical permeability from circular SRR');

xlabel('Freq (Ghz)');

ylabel('Re(mu)'); 


\section{A.2 Theoretical simulation of S-shaped split ring resonator (SRR)}

Listing from source file S-SRR.m

\%S-SRR.m by Neil Patel

\%Given S-shaped SRR parameters, this matlab file calculates the theoretical

$\%$ resonance frequency or frequencies. The center bar may be adjusted to

\%achieve different volumes for $\mathrm{F} 1$ and $\mathrm{F} 2$. A setting of 0.5 means it is in

$\%$ the center and $\mathrm{F} 1=\mathrm{F} 2$. The resonance frequency or frequencies are given and

$\%$ a plot evaluating the permeability relation is output.

\%Calculate resoncnace frequency given unit cell dimensions of an S-SRR.

$\mathrm{h}=4 \mathrm{E}-3 ; \% \mathrm{~S}-\mathrm{SRR}$ width

$\mathrm{w}=7.5 \mathrm{E}-3 ; \% \mathrm{~S}-\mathrm{SRR}$ height

$\mathrm{c}=0.5 \mathrm{E}-3 ; \%$ copper trace width

$\mathrm{d}=0.5 \mathrm{E}-3$; \%distabce between inverted S-shapes

cond_s $=0.5$; \% copper resistance

$\mathrm{a}=10 \mathrm{E}-3$; \%unit cell height

$\mathrm{b}=5 \mathrm{E}-3$; \%unit cell width

$\mathrm{l}=1.0 \mathrm{E}-3$; \%unit cell depth (distance between pairs of S-shapes in H-plane)

$\mathrm{F} 12=(((\mathrm{a} * \mathrm{~b} * 1)-(\mathrm{h} * \mathrm{w} * 1))) /(\mathrm{a} * \mathrm{~b} * 1)$

center_bar $=0.75$

\%calculate volume ratios $\mathrm{F} 1$ and $\mathrm{F} 2$ bases on center bar and S-shape

\%dimensions

$\mathrm{F} 1=($ center_bar $*(\mathrm{~h} * \mathrm{w} * 1)) /(\mathrm{a} * \mathrm{~b} * 1)$;

$\mathrm{F} 2=((1$-center_bar $) *(h * \mathrm{w} * 1)) /(\mathrm{a} * \mathrm{~b} * 1)$;

\% free space constants

epsilon_0 $=8.85 \mathrm{E}-12$;

mu_0 $=1.257 \mathrm{E}-6$;

\%strip capacitances

$\mathrm{Cs}=\left(\right.$ epsilon_0 $\left.0\left(\left(\mathrm{~h}^{*} \mathrm{c}\right) / \mathrm{d}\right)\right)+\left(\right.$ epsilon_0 $\left.*\left(\left(\mathrm{~h}^{*} \mathrm{c}\right) /(\mathrm{l}-\mathrm{d})\right)\right)$;

$\mathrm{Cm}=\mathrm{Cs}$;

$S=a^{*} b$

case when $\mathrm{F} 1=\mathrm{F} 2$.

wm0_squared $=\left(1 /\left(\mathrm{mu}_{-} 0 * \mathrm{~F} 1 * \mathrm{~S}\right)\right) *((\mathrm{l} / \mathrm{Cs})+((2 * \mathrm{l}) / \mathrm{Cm}))$;

$\mathrm{wm} 0=\operatorname{sqrt}\left(w \mathrm{~m} 0 \_\right.$squared $)$;

$f \mathrm{m0}=\mathrm{wmO} /(2 * 3.14159265358979323846)$;

$\mathrm{m}=\mathrm{F} 1 / \mathrm{F} 2$

$\mathrm{n}=\mathrm{Cm} / \mathrm{Cs}$; 
\%resonance frequencies when $\mathrm{F} 1$ does not equal $\mathrm{F} 2$.

$w m 01=\operatorname{sqrt}\left(\left(\left((m+1)^{*}(n+1)+\operatorname{sqrt}\left((m-1)^{\wedge}(2)^{*}\left(n^{\wedge}(2)+\left(2^{*} n\right)\right)+(m+1)^{\wedge}(2)\right)\right) /\left(2^{*} m^{*} n\right)\right)^{*}\left(1 /\left(m u \_0\right.\right.\right.$

$* \mathrm{~S} * \mathrm{~F} 2 * \mathrm{Cs})))$;

$\mathrm{fm01}=\mathrm{wm01} /(2 * 3.14159265358979323846)$;

$w \mathrm{m02}=\operatorname{sqrt}\left(\left(\left((\mathrm{m}+1)^{*}(\mathrm{n}+1)-\operatorname{sqrt}\left((\mathrm{m}-1)^{\wedge}(2)^{*}\left(\mathrm{n}^{\wedge}(2)+(2 * \mathrm{n})\right)+(\mathrm{m}+1)^{\wedge}(2)\right)\right) /\left(2 * \mathrm{~m}^{*} \mathrm{n}\right)\right)^{*}\left(1 /\left(\mathrm{mu} \_0\right.\right.\right.$

* $\mathrm{S} * \mathrm{~F} 2 * \mathrm{Cs})))$;

$\mathrm{fm02}=\mathrm{wm02} /(2 * 3.14159265358979323846)$;

diff $f 0=((\max (f m 01, f m 02)+2 E 9)-(\min (f m 01, f m 02)))$

num_samples $=((\max (\mathrm{fm01}, \mathrm{fm02})+2 \mathrm{E} 9) / 1 \mathrm{E} 9) / 0.01 ;$

step_size $=0.01 \mathrm{E} 9$

$\%$ create frequency points in vector matrix for plotting.

for $\mathrm{a}=1$ :num $\_$samples

freq_matrix $(a, 1)=\left((0)+a^{*}\right.$ step_size $) ;$

end

omega_matrix $=$ freq_matrix.$^{*}\left(2^{*}\right.$ pi);

\%evaluate permeability relation for each frequency point.

$\mathrm{A}=$ omega_matrix $. * \mathrm{mu}_{-} 0 . * \mathrm{~S} . *\left(\mathrm{~F} 1^{\wedge} 2+\mathrm{F} 2 \wedge 2\right) .{ }^{*}$ cond_s.$* 1$;

$\mathrm{B}=(\text { cond_s } . * 1)^{\wedge} 2$;

$\mathrm{C}=\left(\right.$ omega_matrix.$^{*}$ mu_0 $.^{*} \mathrm{~S} . *(\mathrm{~F} 1+\mathrm{F} 2)-(2 . /$ omega_matrix $\left.) *((1 / \mathrm{Cs})+(\mathrm{l} / \mathrm{Cm}))\right)$

.$^{*}$ cond_s $* 1$

if $(F 1=F 2)$

mu_eff_num $=\left(\right.$ omega_matrix.$^{*}$ mu_0 $\left.*^{*} \mathrm{~S}\right) .{ }^{\wedge} 2{ }^{*} \mathrm{~F} 2{ }^{*} \mathrm{~F} 1 *^{*}(\mathrm{~F} 1+\mathrm{F} 2)-\left(\mathrm{mu}_{-} 0 * \mathrm{~S}\right) .^{*}$

$\left((\mathrm{F} 1 \wedge 2+\mathrm{F} 2 \wedge 2) *(1 / \mathrm{Cs})+(\mathrm{F} 1-\mathrm{F} 2)^{\wedge} 2 *(\mathrm{~V} / \mathrm{Cm})\right)+(\mathrm{i} * \mathrm{~A}) ;$

mu_eff_den $=($ omega_matrix $* *$ mu_0 $* \mathrm{~S}) .^{\wedge} 2 *^{*} \mathrm{~F} 1 *^{*} \mathrm{~F} 2-\left(\mathrm{mu} \_0 * \mathrm{~S}\right) \cdot *(\mathrm{~F} 1+\mathrm{F} 2) .^{*}$

$((\mathrm{l} / \mathrm{Cs})+(\mathrm{l} / \mathrm{Cm}))+\left(\left(1 . /\left((\right.\right.\right.$ omega_matrix $\left.\left.\left.) .{ }^{\wedge} 2\right)\right) *(\mathrm{l} / \mathrm{Cs})\right) \cdot *((1 / \mathrm{Cs})+((2 * \mathrm{l}) . /(\mathrm{Cm})))-\mathrm{B}+\left(\mathrm{i} \cdot{ }^{*} \mathrm{C}\right) ;$

end

if $(\mathrm{F} 1==\mathrm{F} 2)$

$\mathrm{X}=\left(\right.$ omega_matrix $\left.* \mathrm{mu}_{-} 0 *{ }^{*} \mathrm{~F} 1 . * \mathrm{~S}\right) . \sim 2 *\left(1-\left(1 . /\left(\right.\right.\right.$ omega_matrix.${ }^{*}$ mu_0 $\left.\left.0 * \mathrm{~F} 1 . * \mathrm{~S}\right)\right)$ $\left.{ }^{*}(\mathrm{~V} / \mathrm{Cs})\right)$;

$\mathrm{D}=\mathrm{A} . / \mathrm{X}$

$\mathrm{E}=\mathrm{B} . / \mathrm{X}$

$\mathrm{G}=\mathrm{C} . / \mathrm{X}$

mu_eff_num $=2 * \mathrm{~F} 1+\mathrm{i} * \mathrm{D}$;

mu_eff_den $=1-\left(1 . /\left((\right.\right.$ omega_matrix $\left.\left.) .{ }^{\wedge} 2 *^{*} \mathrm{mu}_{-} 0 *^{*} \mathrm{~F} 1{ }^{*} \mathrm{~S}\right)\right) \cdot{ }^{*}((\mathrm{l} / \mathrm{Cs})+((2 * \mathrm{l}) / \mathrm{Cm}))-\mathrm{E}+$ $(\mathrm{i} . * \mathrm{G})$; 


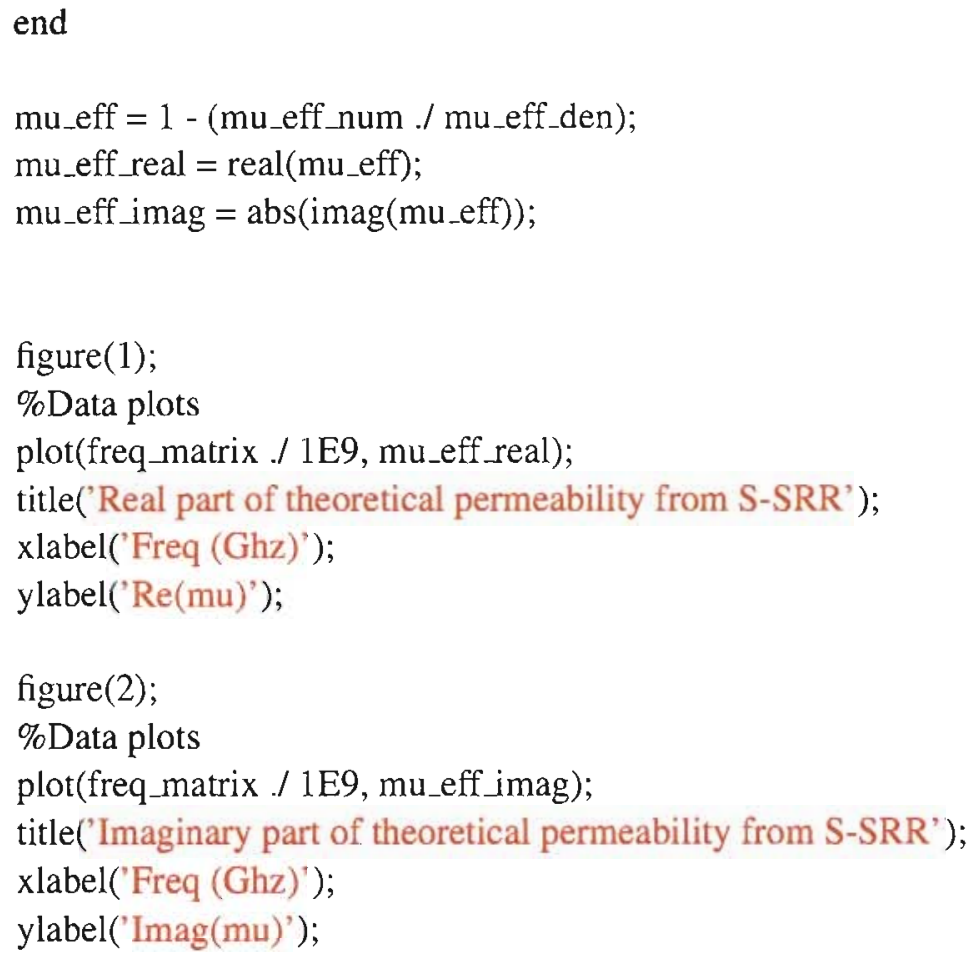

\section{A.3 Parameter extraction using Ziolkoswki's modified NRW relations}

Listing from source file NRW1.m.

\% Implementation of Ziolkowski's version of the NRW algorithm

$\%$ Neil Patel

\%MSEE Thesis

\%Determine complex S-parameters

$\mathrm{S} 11=\mathrm{S} 11 \mathrm{Mag} * \exp (\mathrm{j} *(3.141 * \mathrm{~S} 11 \mathrm{Phase} / 180))$

S21 = S21Mag . $* \exp (\mathrm{j} *(3.141 * \mathrm{~S} 21 \mathrm{Phase} / 180)) ;$

$\% \mathrm{An}$ arbitrary value is chosen for the thickness of the metamaterial slab.

$\% \mathrm{~d}=0.1$;

$\% \mathrm{~d}=0.007366$

$\mathrm{d}=0.01$;

$\%$ Sum and difference equations for scattering parameters

$$
\begin{aligned}
& \mathrm{V} 1=\mathrm{S} 21+\mathrm{S} 11 ; \\
& \mathrm{V} 2=\mathrm{S} 21-\mathrm{S} 11 ;
\end{aligned}
$$

\%freespace wavenumber $\mathrm{k} 0=(2 * 3.141 *$ Freq * 1e9)./ $3 \mathrm{e} 8$ 
\%calculate complex permeability using Ziolkowski's equations

$\mathrm{mu} \_1 \_\mathrm{den}=\mathrm{j} \cdot * \mathrm{k} 0 * \mathrm{~d}$;

mu_r1 $=2 . /$ mu_r1_den;

mu_r2_num $=1-\mathrm{V} 2$;

mu_r2_den $=1+\mathrm{V} 2$;

mu $\_2=$ mu_r2_num ./ mu $r 2 \_d e n ;$

$\mathrm{mu} \_\mathrm{r}=\mathrm{mu} \_r 1 . * \mathrm{mu} \_\mathrm{r} 2$

\%Break complex mu into real and imaginary parts

real_mu_r $=\operatorname{real}\left(\mathrm{mu} \_r\right)$;

imag_mu $r=\operatorname{imag}(m u r)$;

mag_mu $r=\operatorname{sqrt}\left(\left(r e a l \_m u \_r . \wedge 2\right)+(\right.$ imag_mu_r .^2));

wave_imp_squared_num $=((S 11+1) . \wedge 2)-S 21 . \wedge 2$;

wave_imp_squared_den $=((\mathrm{S} 11-1) . \wedge 2)-\mathrm{S} 21 . \wedge 2$;

wave_imp_squared $=$ wave_imp_squared_num ./ wave_imp_squared_den;

wave_imp $=$ sqrt(wave_imp_squared);

gamma $=\left(\right.$ wave $\_$imp -1$) . /($ wave_imp +1$)$;

$\mathrm{k}=(1 /(\mathrm{j} * \mathrm{~d})) *(((1-\mathrm{V} 1) *(1+$ gamma $)) . /(1-($ gamma $* \mathrm{~V} 1))) ;$

thickness $=\operatorname{real}(\mathrm{k}) . * \mathrm{~d}$;

\%Use Ziolkowski's equations to find permittivity epsilon.

epsilon $\_3=m u \_r+(\mathrm{j} *((2 * \mathrm{~S} 11) . /(\mathrm{k0} * \mathrm{~d})))$;

epsilon_r2 $=\left(2 . /\left(\mathrm{j} . * \mathrm{k} 0 *^{*} \mathrm{~d}\right)\right) *^{*}((1-\mathrm{V} 1) . /(1+\mathrm{V} 1))$;

epsilon_rl $=\left((\mathrm{k} . / \mathrm{k} 0) .{ }^{\wedge} 2\right) *\left(1 . / \mathrm{mu} \_r\right)$;

real_epsilon_r1 = real(epsilon $r 1)$;

imag_epsilon_rl = imag(epsilon_r1);

real_epsilon_r2 = real(epsilon_r2);

imag_epsilon_r2 = imag(epsilon_r2);

real_epsilon_r3 = real(epsilon_r3);

imag_epsilon_r3 = imag(epsilon_r3);

$\mathrm{n} 1=\mathrm{k} . / \mathrm{k} 0$

real $\_\mathrm{n} 1=\operatorname{real}(\mathrm{n} 1)$;

imag_n1 = imag(n1);

$\mathrm{n} 2=\operatorname{sqrt}\left(\right.$ epsilon $r \mathrm{r} 1 . * \mathrm{mu} \_$);

real $\_n 2=\operatorname{real}(\mathrm{n} 2)$;

imag_n2 = imag(n2); 


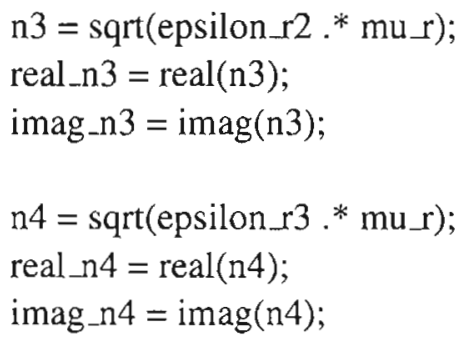

figure(1);

$\%$ Data plots

plot(Freq, real_mu_r);

title('Real part of extracted permeability from S-parameters');

xlabel('Freq (Ghz)');

ylabel('Re(mu)');

figure(2);

plot(Freq, real_epsilon_rl);

title('Real part of extracted permittivity (eqn1) from S-parameters');

xlabel('Freq (Ghz)');

ylabel('Re(espilon)');

figure(3);

plot(Freq, real_epsilon_r2);

title('Real part of extracted permittivity (eqn2) from S-parameters');

xlabel('Freq (Ghz)');

ylabel('Re(espilon)');

figure(4);

plot(Freq, real_epsilon_r3);

title('Real part of extracted permittivity (eqn3) from S-parameters');

xlabel('Freq (Ghz)');

ylabel('Re(espilon)');

figure(5);

plot(Freq, real_n1);

title('Real part of extracted index of refraction from S-parameters');

xlabel('Freq (Ghz)');

ylabel('Re(espilon)');

figure(6);

plot(Freq, imag_n1);

title('Imaginary part of extracted index of refraction from S-parameters'); 


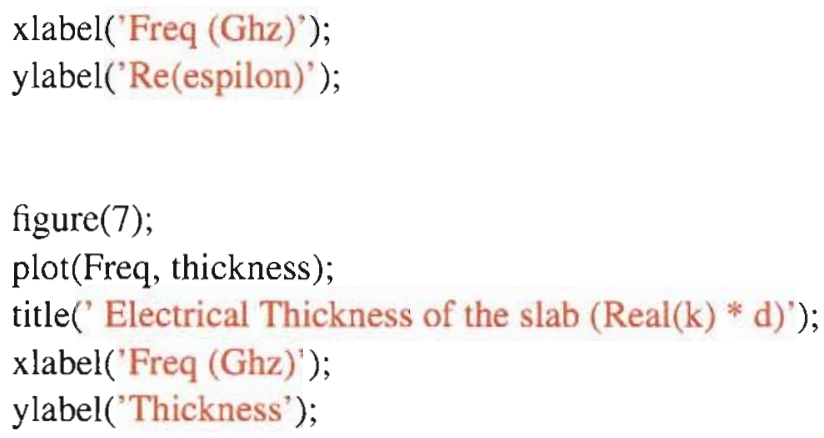

\section{A.4 Parameter extraction using Chen et al's modified NRW relations}

This section provides Matlab code implementing a basic form of the algorithm proposed in [16] to extract bulk parameters from metamaterial structure simulations. While the theory and constraints used in this paper to address issues with the original Nicolson-Ross-Weir (see section 2.2) equations is not included in this thesis, the matlab code below performs parameter extraction and is included here to provide additional information and avenues of research for the reader. Listing from source file NRW2.m

$\%$ Implementation of Chen et al's modified version of the Nicolson Ross Weir $\%$ Method for paramater extraction

$\%$ Neil Patel

$\%$ MSEE Thesis

$\%$ Determine complex $S$-parameters

S11 = S11Mag * $\exp (\mathrm{j} *(3.141 *$ S11Phase/180));

$\mathrm{S} 21=\mathrm{S} 21 \mathrm{Mag} * \exp (\mathrm{j} *(3.141 * \mathrm{~S} 21 \mathrm{Phase} / 180))$

\%An arbitrary value is chosen for the thickness of the metamaterial slab.

\%Should match thickness of slab used in HFSS simulations.

$\mathrm{d}=0.01$

\%Sum and difference equations for scattering parameters

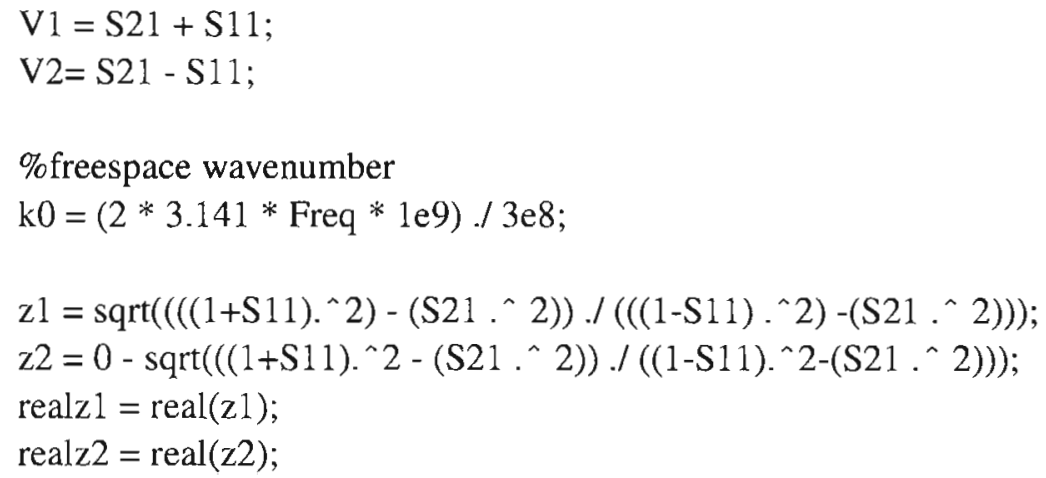




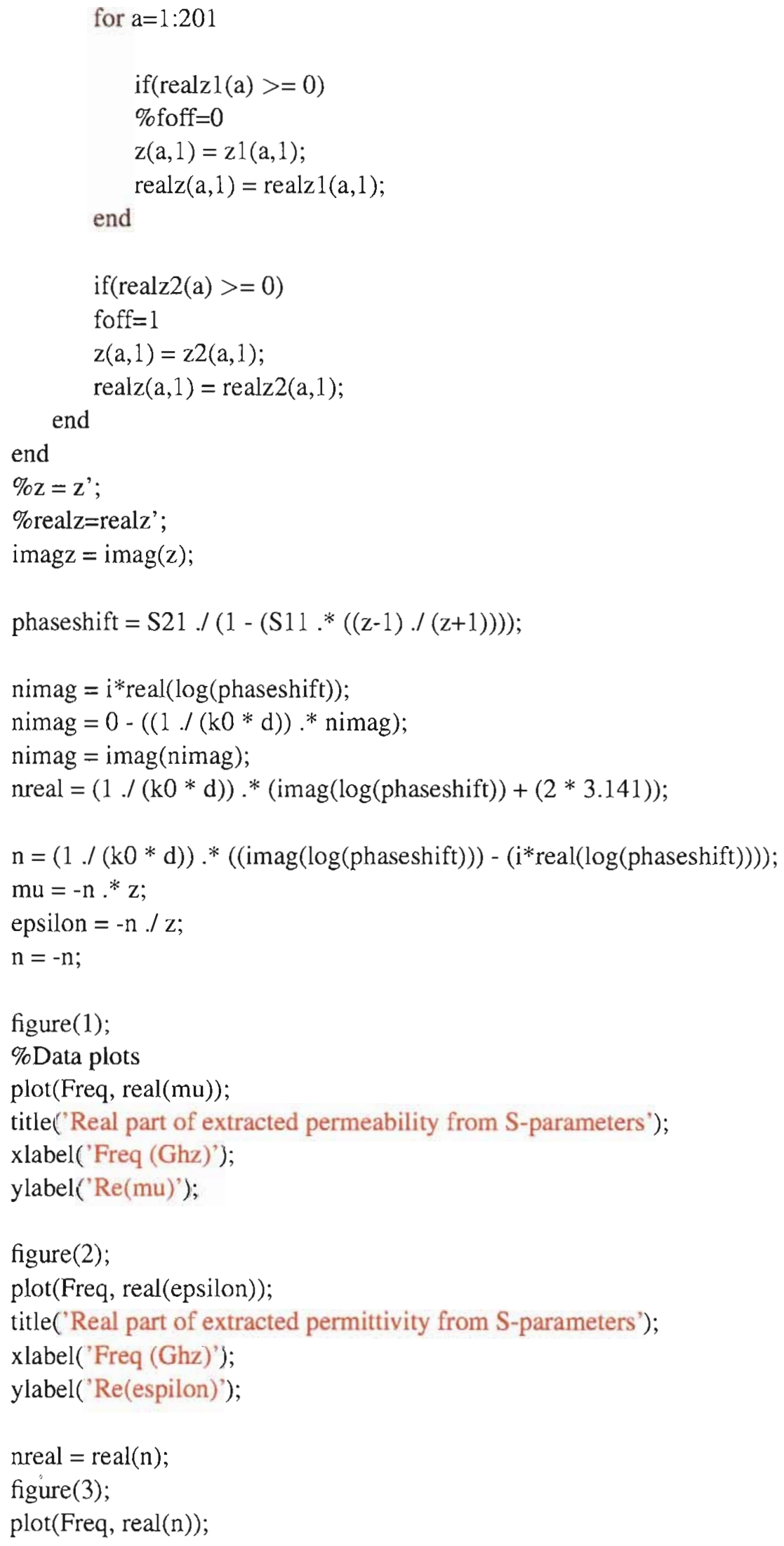


title('Real part of extracted index of refraction from S-parameters'); xlabel('Freq (Ghz)');

ylabel('Re(n)'); 


\section{Appendix B}

\section{Analysis of Project Design}

\section{B.1 Summary of Functional Requirements}

The metamaterial design presented in this design is a culmination of recent research in the field of metamaterial design for Microwave applications. The transmission slab design presented in this thesis successfully displays band-pass characteristics for an illuminating plane wave normal to the entrance face with a target of peak transmission at $8.5 \mathrm{GHz}$. The metamaterial prism successfully displays negative refraction in the $8 \mathrm{GHz}$ to $9 \mathrm{GHz}$ frequency range and thus may be used as a frequency dependent beam director in a system containing multiple receivers offset spatially along the circumference of an imaginary circle boundary. This is one potential application of a metamaterial prism in altering the emitting beam direction based on the illuminating frequency.

\section{B.2 Primary constraints}

- Lossy material - FR4 epoxy board is used in the majority of manufactured PCB boards for electronics applications. At microwave frequencies it displays increased dielectric loss due to a high dielectric loss tangent $\delta$ of 0.02 at $10 \mathrm{GHz}$. While relatively cheap and easy to obtain, the dielectric loss in the FR4 prevents the transmission characteristics of a metamaterial fabricated with FR4 epoxy dielectric from perfectly matching to free-space (0dB transmission). Rogers Duroid 8550 was considered in this project, however for large scale structures where fifty or more boards need fabrication, the increased cost of the boards may make such a solution impractical.

- Milling machine - A milling machine is available for use in the Cal Poly Antenna Lab. However, it was found during initial fabrication of the PCB metamaterial boards that it is an impractical solution for large scale fabrication. The milling bits wear out very quickly and the continuous action of replacing bits is expensive. Furthermore, the milling of a single board may take 30 to 45 minutes. During the course of the project several hundred boards were required. Such a process is not time effective and so board layout files were supplied to an external manufacturer for metamaterial PCB board fabrication. 


\section{B.3 Economic}

- Estimated FR-4 epoxy board fabrication was approximately $\$ 1000$. This figure increased when it was found that the milling machine in the Cal Poly Antenna lab was not a practical solution for large scale board fabrication.

- Actual costs for board fabrication for 2 transmission slab designs and a metamaterial prism totaled $\$ 1693.67$.

\section{B.3.1 Bill of Materials}

Current Composites order:

\begin{tabular}{||c|c|c|c||}
\hline Quantitiy & Description & Per unit cost & Total cost \\
\hline 20 & 12 x 9" x 61 mils 1/2 Oz. Copper Double side clad FR4 & 3.92 & 78.40 \\
\hline 20 & $12 " \times$ 9" x 31 mils 1/2 Oz. Copper Single side clad FR4 & 2.15 & 43.00 \\
\hline 10 & 12 " x 9" x 31 mils 1/2 Oz. Copper Double side clad FR4 & 2.60 & 26.00 \\
\hline 16 & 12 " x 9" x 31 mils No Clad FR4 & 1.73 & 27.68 \\
\hline & & SUBTOTAL & $\$ 175.08$ \\
\hline & & TAX & $\$ 12.69$ \\
\hline & & TOTAL & $\$ 187.77$ \\
\hline
\end{tabular}

Table B.1 Bill of materials for products ordered from Current Composites.

The PCB company order:

\begin{tabular}{||c|c|c|c||}
\hline Quantitiy & Description & Per unit cost & Total cost \\
\hline 1 & Quote 4701: & 251.25 & $\$ 251.25$ \\
& $\begin{array}{c}9026 \mathrm{~mm} \times 130 \mathrm{~mm} \text { FR4 PCB boards, single sided. } \\
\text { Plus engineering setup and photplotting film. }\end{array}$ & \\
\hline 1 & $\begin{array}{c}10026 \mathrm{~mm} \times 130 \mathrm{~mm} \text { FR4 PCB boards, doubles sided. } \\
\text { Plus engineering setup and photplotting film. }\end{array}$ & 401.12 & $\$ 401.12$ \\
\hline 1 & $\begin{array}{c}\text { Quote 4704: } \\
\text { 20 257.5 x 267.5mm double sided panels (prism designs) } \\
\text { Plus engineering setup and photplotting film. }\end{array}$ & 861.3 & $\$ 861.3$ \\
\hline
\end{tabular}

Table B.2 Bill of materials for products ordered from the PCB company.

\section{B.4 Environmental}

There is no immediate environmental impact associated with the use of metamaterials in Microwave applications. The impact of manufacturing the PCB boards is also presumed to be minimal. No solder is used in the manufacturing of the boards, thus no tin-lead alloy waste is generated through the fabrication of the metamaterial PCB boards. Furthermore, there are no plated through-holes in the PCB board. The creation of such plated through holes involves the electroless deposition of 
copper from aqueous solutions containing chelates, formaldehyde and copper. Such a process may produce unwanted waste. Generally, the PCB industry has moved to improving the environmental impact of its manufacturing process including reduced water consumption which was previously treated as an unlimited resource.

\section{B.5 Manufacturability}

It has been reported that $\mathrm{PCB}$ board milling is a lengthy and expensive process. Bulk orders of metamaterial PCB boards are easily manufactured at professional manufacturers employing methods such as etching. However, some restrictions are placed on the designs including trace width and distance between traces. The 'PCB Company', chosen to manufacture the PCB boards for the metamaterial structures placed a restriction on a minimum trace width of $10 \mathrm{mils}(0.254 \mathrm{~mm})$ and spacing width of $8 \mathrm{mils}(0.2 \mathrm{~mm})$. The manufacture constraints due to the limits in the process used by the manufacturer impact the minimum limits in sizings for the metamaterial inclusions.

\section{B.6 Sustainability}

The completed structure is rigid when fabricated with dielectric rods or epoxy glue used to connect the boards together. There are no immediate issues or challenges associated with maintaing the completed device. The structures may be upgraded in terms of size, however the inclusion design cannot be changed. Thus, to target a different operating frequency, all metamaterial inclusions must be be redesigned and all PCB boards re-manufactured.

\section{B.7 Ethical}

There are no immediate ethical implications associated with the metamaterial structures fabricated in this project.

\section{B.8 Health and safetey}

There are no immediate health and safety issues associated with the metamaterial structures fabricated in this project.

\section{B.9 Social and political}

There are no immediate social or political issues associated with the metamaterial structures fabricated in this project.

\section{B.10 Development}

The finite element 3D electromagnetic solver, HFSS was used in the development of the metamaterial strucures. HFSS was used to not only characterize the copper inclusions but also confirm the metamaterial structures frequency of operation and refraction characteristics. This commercial tool 
used extensively in industry for EM simulations and antenna design is not taught in class but is available to students working on projects in the Cal Poly RF and Antennas group. 


\section{Bibliography}

[1] J C Bose, On the rotation of plane of polarisation of electric waves by a twisted structure, Proc. Roy. Soc. vol63, pp146-152, 1898.

[2] I V Lindell A H Sihvola Karl F. Lindman: The last Hertizan and and a harbinger of electromagnetic chirality, IEEE Antennas Prop. Mag. vol.34, no.3 pp24-30, 1992.

[3] W E Kock, Metallic delay lenses. Bell Sys, Tech J. vol 27, pp58-82, 1948.

[4] V. G. Veselago, The electrodynamics of substances with simultaneously negative values of epsilon and mu. Sov. Phys. Uspekhi, vol. 10, no. 4, pp. 509-514, Jan-Feb. 1968.

[5] R W Ziolkowksi, E Heyman, Wave propagation in media having negative permittivity and permeability, Phys. Rev. E, Vol. 64, 056625, 2001.

[6] D Pozar, Microwave engineering 3rd Edition, Wiley, 2005.

[7] D R Smith, N Kroll, Negative Refractive index in Left-handed Materials Phys. Rev Letters, Vol.85 No. 14, Oct 2000.

[8] R. A. Shelby, D. R. Smith, and S. Schultz, Experimental verification of a negative refractive index of refraction, Sci., vol. 292, pp. 77-79, Apr. 2001.

[9] D R Smith, W Padilla, D C Vier, S C Nemat-Nasser, S Schultz, Negative permeability from Split ring resonator arrays, IEEE 2000.

[10] R.W Ziolkowski, Design Fabrication and testing of double negative metamaterials IEEE Trans. Antennas and Prop., vol. 51, no. 7, JULY 2003

[11] N V. Kantartzis, D L. Sounas, C S Antonopolous, and T D Tsiboukis, A wideband ADI-FDTD Algorithm for the Design of double negative metamaterial based waveguides and antenna substrates, IEEE Trans. On Antenna Prop., Vol 43, no. 4, April 2007

[12] A.M.Nicolson, G.F.Ross, Measurement of the Intrinsic Properties of Materials by Time domain techniques, IEEE Trans. Instrum and Meas.Vol 19, Nov 1970

[13] J. Baker-Jarvis, M D Janezic, R G Geyer Transmission/Reflection and Short-circuit line methods for measuring permittivity and permeability. National Inst. Standards Technology (NIST). DEC 1993.

[14] E Ni An Uncertainty analysis for the measurement of intrinsic properties of materials by combined transmission-reflection method. IEEE. Trans. Instrumentation and Meas. Aug 1992. 
[15] J Shenhui, D Ding, J Quanxing Measurement of electromagnetic properties of materials using transmission/reflection method in coaxial line. Asia-Pacific Conf. Environmental Electromagnetics CEEM'2003, Nov.4-7 2003.

[16] X Chen, T M Grzegorczyk, B Wu, J Pacheo, J A Kong Robust method to retrieve the constitutive effective parameters of metamaterials. Phys Rev. E 70, 016608, 2004.

[17] J B Pendry, A J Holden, D J Robbins and W J Stewart, Magnetism from conductors and other nonlinear phenomena, IEEE Trans. Microwave Theory, Vol 47, No. 11, Nov. 1999.

[18] J B Pendry et al, Low frequency plasmons in thin-wire structures, J. Phys.: Condens. Matter $104785-4809$

[19] W Rotman, Plasma simulation by artificial dielectrics and parallel-plate media, IRE Trans. Antenna Prop. Jan. 1962.

[20] P Ikonen et al Experimental demonstration of sub-wavelength field channeling at microwave frequencies using a capacitive loaded wire medium. Phys. Rev. B 73, 073102, (2006).

[21] K Aydin and E Ozbay, Negative refraction through an impedance matched left-handed metamaterial slab. J. Opt. Soc. Am. B Vol.23, No.3 March 2006.

[22] F Zhang, G Houzet, S Potet, E Lheurette, M Chaubet and D Lippens, Metamaterials-based routing devices for space applications, IEEE, 2007.

[23] A Alu, N Engheta, A Erentok, R W Ziolkowski, Single-negative, double-negative, and low-index metamaterials and their electromagnetic applications, IEEE Antennas Prop. Mag. Vol.49, No.1, Febr 2007.

[24] D R Jackson, T Zhao, J T Williams, A A Oliner, Leaky surface-plasmon theory for dramatically enhanced transmission through a sub-wavelength aperture Parts I-III, IEEE, 2003.

[25] L Zhou, H Li, Y Qin, Z Wei, C Chan, Directive emissions from subwavelength metamaterialbased cavities. Appl. Phys. Letters 86, 101101 (2005).

[26] S Enoch, G Tayeb, P Sabouroux, N Guerin, P Vincent, A metamaterial for directive Emission, Phys. Rev Letters, Vol89, No.21, Nov. 2002.

[27] A Alu, F Bilotti, N Engheta, L Vegni, Metamaterial covers over a small aperture. IEEE, trans. Antenna Prop. Vol54, No.6, June 2006.

[28] P Ikonen Modeling and Analysis of composite Antenna Superstrates consisting on grids of loaded wires, Phys Rev. B 070103, May 2007.

[29] P Ikonen Artificial Dielectrics and Magnetics in Microwave engineering: A brief historical revision. Helsinki University of Technology.

[30] H S Chen, L X Ran, J T Huangfu, X M Zhang and K S Chen, Left-handed metamaterials composed of only S-shaped resonators. Phys. Rev. E, vol.70, 057605, 2004.

[31] H S Chen, L X Ran, J T Huangfu, X M Zhang and K S Chen, Magnetic properties of S-shaped split ring resonators, PIER 51, 231-247, 2005. 
[32] D R Smith, J J Mock, A F Starr, D Schurig, A gradient index metamaterial. July, 2004.

[33] Microwave Mathematics, http://www.microwaves101.com/encyclopedia/waveguidemath.cfm 